\title{
UMA ANÁLISE BAYESIANA PARA UM GRUPO DE EXPERIMENTOS COMPLETAMENTE \\ ALEATORIZADOS
}

\author{
JOÃO GIL DE LUNA \\ Estatístico
}

Orientador: Prof. Dr. HUMBERTO DE CAMPOS

\begin{abstract}
Tese apresentada à Escola Superior de Agricultura "Luiz de Queiroz", da Universidade de São Paulo, para obtenção do título de Doutor em Agronomia, Área de Concentração: Estatística e Experimentação Agronômica.
\end{abstract}

P I R A C I C A B A

Estado de São Paulo - Brasil

Janeiro - 1996 
Dados Internacionais de Catalogação na Publicação (CIP)

DIVISÃO DE BIBLIOTECA E DOCUMENTAÇĂO - Campus "Luiz de Queiroz"/USP

Luna, João Gil de

Uma análise bayesiana para um grupo de experimentos completamente aleatorizados / João Gil de Luna. - - Piracicaba, 1996.

$129 \mathrm{p}$.

Tese (doutorado) - - Escola Superior de Agricultura Luiz de Queiroz, 1996.

Bibliografia.

1. Experimento agricola - Método estatístico 2. Método de Bayes I. Título

CDD 519.542 


\section{UMA ANÁLISE BAYESIANA PARA UM GRUPO DE EXPERIMENTOS COMPLETAMENTE ALEATORIZADOS}

JOÃO GIL DE LUNA

Aprovado em: 06/03/96

Comissão julgadora:

Prof. Dr. Humberto de Campos

ESALQ/USP

Prof. Dr. Décio Barbin ESALQ/USP

$\operatorname{Prof}^{a} \operatorname{Dr}^{a}$. Clarice Garcia Borges Demétrio ESALQ/USP

Prof. Dr. Sérgio do Nascimento Kronka FCAV/UNESP

Prof. Dr. João Riboldi UFRS/PORTO ALEGRE

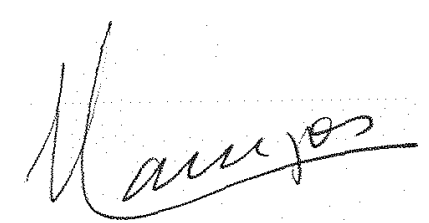

Prof. Dr. Humberto de Campos Orientador 


\section{DEDICAÇÃO}

A meus pais e irmãos, com gratidão,

Ao mano Leandro(in memorian), com saudade.

À Lili, Iviane e Igor, sentido do que faço,

dedico com carinho e amor. 


\section{AGRADECIMENTOS}

Ao Professor Humberto de Campos, pela orientação e estímulo.

Aos Professores Doutores Décio Barbin, Clarice Garcia Borges Demétrio, Antônio Francisco Iemma, Maria Cristina S. Nogueira e José Eduardo Corrente, pela amizade e consideração.

Aos Professores do Departamento de Matemática e Estatística da ESALQ/USP, pelos ensinamentos e convívio.

Aos Colegas de curso, Claudia, Doris, Imaculada, Liciana, Luis, Pillar, Roseli e Silvio, pelo companheirismo e amizade.

À minha família, Lili, Iviane, Igor, por ter contornado a minła ausência.

Às secretárias do Departamento de Matemática e Estatística da ESALQ/USP, nas pessoas de Rosa e Solange, por terem me suportado.

Aos Colegas Professores do DME/UFPB-CAMPUS II, pela confiança e oportunidade que me deram para realização deste curso.

Ao Pesquisador Antônio Fernando Sobral da Costa, por ter cedido os dados experimentais usados neste trabalho.

A todos aqueles que, direta ou indiretamente, colaboraram com esta importante etapa da minha vida. 


\section{SUMÁRIO}

\section{INTRODUÇÃO}

2 REVISÃO DE LITERATURA

2.1 Análise de Grupo de Experimentos . . . . . . . . . . . . . 5

2.2 A Natureza do Método Bayesiano . . . . . . . . . . . . . . 7

2.2 .1 O Teorema de Bayes . . . . . . . . . . . . . . 7

2.2.2 O Teorema de Bayes e a Função de Verossimilhança . . . . . . 8

2.2.3 A Natureza Sequencial do Teorema de Bayes . . . . . . . . . . 9

2.3 Inferència Bayesiana Básica . . . . . . . . . . . . . . . . 10

2.4 A Informaçāo a Priori . . . . . . . . . . . . . . . . . . 11

2.5 Modelos Lineares Bayesianos . . . . . . . . . . . . . . 15

2.6 Testes Bayesianos de Homogeneidade de Variàncias . . . . . . . . . . 16

3 DESENVOLVIMENTO TEÓRICO 17

3.1 Generalidades . . . . . . . . . . . . . . . 17

3.2 As Análises Individuais . . . . . . . . . . . . . . . . . . 18

3.2 .1 O Modelo Estatístico . . . . . . . . . . . . . . 18

3.2 .2 A Função de Verossimilhança . . . . . . . . . . . . . . 19

3.2.3 A Função de Distribuição a Priori de $\left(\boldsymbol{\theta}, \sigma^{2}\right)$. . . . . . . . . 21

3.2.4 A Função de Distribuição Conjunta a Posteriori de $\left(\boldsymbol{\theta}, \sigma^{2}\right)$. . 21

3.2.5 Distribuição Marginal a Posteriori de $\sigma^{2} \ldots \ldots . . . . . .22$

3.2 .6 A Distribuição Marginal a Posteriori de $\boldsymbol{\theta}$. . . . . . . . . 23

3.2.7 Região de Alta Densidade a Posteriori . . . . . . . . . . . . 24

3.2.8 Propriedades da Distribuição a Posteriori de $\boldsymbol{\theta}$. . . . . . . . 25

3.2.9 Inferèncias Conjunta e Marginal . . . . . . . . . . . . 29

3.2.10 Comparação das Dispersões de K Populações Normais . . . . . 31

3.3 A Análise Conjunta de $J$ Experimentos . . . . . . . . . . . . . 33 
$3.3 .1 \quad$ O Modelo Estatístico . . . . . . . . . . . . . . . . . . . 34

3.3.2 A Função de Verossimilhança . . . . . . . . . . . . . 35

3.3.3 A Distribuição a priori de $\left(\bar{\theta}, \phi, \sigma_{e}^{2}, \sigma_{e g}^{2}, \sigma_{e g b}^{2}\right) \ldots \ldots \ldots 40$

3.3.4 A Distribuição a Posteriori de $\left(\bar{\theta}, \boldsymbol{\phi}, \sigma_{e}^{2}, \sigma_{e g}^{2}, \sigma_{e g b}^{2}\right) \ldots \ldots . . .40$

3.3.5 Distribuição Marginal a Posteriori dos Componentes da Variância $\left(\sigma_{e}^{2}, \sigma_{g}^{2}, \sigma_{b}^{2}\right) \ldots \ldots \ldots \ldots \ldots \ldots$

3.3.6 Distribuição Marginal a Posteriori de $\phi \ldots . . . . . .44$

3.3.7 Aproximando a Distribuição $p(\boldsymbol{\phi} \mid \boldsymbol{y}) \ldots \ldots \ldots \ldots$

3.3.8 Inferências Conjuntas e Marginais Sobre $\phi$ na Análise do Grupo de $J$ Experimentos Completamente Aleatorizados . . . . 51

3.3.9 Distribuição Marginal a Posteriori de $\sigma_{e}^{2} \ldots \ldots \ldots$. . . 53

3.3.10 Composição das Estimativas das Variâncias para Estimar $\sigma_{e}^{2}$. 59

3.3.11 Distribuição Marginal a Posteriori de $\sigma_{g}^{2} \ldots \ldots \ldots$. . . 59

3.3.12 Distribuição Marginal a Posteriori de $\sigma_{b}^{2} \ldots \ldots \ldots$. . . 60

3.4 Resumo da Teoria . . . . . . . . . . . . . . . . . . . . . 63

3.4.1 Análises Individuais . . . . . . . . . . . . . . . . . . 63

3.4.2 A Análise Conjunta . . . . . . . . . . . . . . . . 65

3.4.3 Distribuição Marginal a Posteriori dos Componentes da Variância, $\sigma_{e}^{2}, \sigma_{g}^{2}$ e $\sigma_{b}^{2} \ldots \ldots \ldots \ldots 66 \ldots \ldots \ldots$

\section{EXEMPLO ILUSTRATIVO 68}

4.1 A Origem dos Dados e Hipóteses de Interesse . . . . . . . . . . 68

4.2 Os Dados Experimentais . . . . . . . . . . . . . . . . 70

4.3 As Análises Individuais . . . . . . . . . . . . . . . . . . . . . . . 72

4.3.1 A Homogeneidade das Variâncias Dentro dos Ensaios . . . . 72

4.3.2 As Distribuições Marginais a Posteriori de $\sigma^{2}, p\left(\sigma^{2} \mid \boldsymbol{y}\right)$, Relativas a Cada Experimento . . . . . . . . . . . 75

4.3.3 Distribuição Conjunta a Posteriori para os Efeitos Médios dos Tratamentos, $p(\boldsymbol{\theta} \mid \boldsymbol{y})$, em Cada Experimento . . . . . . . 77

4.3.4 Distribuição Marginal a Posteriori de $\theta_{i}, p\left(\theta_{i} \mid \boldsymbol{y}\right) \ldots \ldots .78$ 
4.3.5 Comparaçōes dos Efeitos Médios dos Tratamentos . . . . . . . 80

4.3.6 Distribuiçōes Marginais a Posteriori dos Contrastes $\phi_{j}, p\left(\phi_{j} \mid \boldsymbol{y}\right) 81$

4.4 A Análise Conjunta dos Experimentos . . . . . . . . . . . . . . . 84

4.4.1 A Distribuição a Posteriori Aproximada de $\phi, p(\phi \mid y) \ldots . . .87$

4.4.2 Inferèncias Conjunta e Marginais Sobre $\phi$ na Análise Conjunta 88

4.4.3 Ajuste dos Graus de Liberdade e Quadrados Médios para Construção das Densidades a Posteriori dos Componentes da Variância ................... . 90

4.4.4 Inferência Marginal a Posteriori Sobre $\sigma_{e}^{2}$. . . . . . . . . 90

4.4.5 Inferência Marginal a Posteriori Sobre $\sigma_{g}^{2}$. . . . . . . . . . 92

4.4.6 Inferència Marginal a Posteriori Sobre $\sigma_{b}^{2} \ldots \ldots . . . . . . .93$

4.5 A Análise Clássica . . . . . . . . . . . . . . . . . . . . 95

4.5.1 As Análises Individuais Clássicas . . . . . . . . . . . . . . . 95

4.5.2 A Análise Conjunta Clássica . . . . . . . . . . . . . . . 96

5 CONCLUSÕES 104

6 APÊNDICE 111 


\title{
UMA ANÁLISE BAYESIANA PARA UM GRUPO DE EXPERIMENTOS COMPLETAMENTE ALEATORIZADOS
}

\author{
Autor: João Gil de Luna \\ Orientador: Prof. Dr. Humberto de Campos
}

\section{RESUMO}

Em experimentaçāo agronômica é muito freqüente a estruturação da análise estatística de um grupo de experimentos. Os motivos que levam a esta prática, quase sempre, estão associados ao desejo dos pesquisadores em obter conclusões mais gerais para os tratamentos envolvidos, bem como, acerca dos fatores ambientais (ano agrícola, estaçòes do ano, épocas, regiões, tipos de solo, etc.) e da interação (tratamento) $\times$ (experimento).

Este estudo apresenta uma estratégia bayesiana para analisar um grupo de experimentos completamente aleatorizados, onde o interesse do pesquisador é obter conclusões genéricas acerca do fator tratamento, considerado de efeito fixo, e num segundo plano, obter informações sobre os componentes da variància devidos ao fator experimento e à interaçāo (tratamento) $\times$ (experimento), considerados de efeitos aleatórios.

Nas análises individuais é adotado o modelo de médias de caselas, dado por $y_{i r}=\theta_{i}+e_{i r}$, onde $y_{i r}$ é a $r$-ésima observação obtida da unidade experimental que recebeu o tratamento $i, \theta_{i}$ é o efeito médio do $i$-ésimo tratamento e $e_{i r}$ é o erro experimental associado à observação $y_{i r}$. Além disso, supõe-se que $e_{i r}$ é distribuído como $N\left(0, \sigma^{2}\right)$. $\mathrm{Na}$ análise conjunta é adotado um modelo misto, isto é, $y_{i j r}=\theta_{i}+\beta_{j}+\gamma_{i j}+e_{i j r},(i=1, \ldots, K ; \quad j=1, \ldots, J ; \quad r=1, \ldots, R)$, onde $y_{i j r}$ é a $r$-ésima observação que recebeu o tratamento $i$ no experimento $j, \beta_{j}$ é o efeito do 
j-ésimo experimento, $\gamma_{i j}$ é o efeito da interação do nível $i$ do fator tratamento com o j-ésimo experimento, $e_{i j r}$ é o erro experimental associado à observação $y_{i j r}$ e $\theta_{i}$ como já definido. Ademais, supòe-se que $\epsilon_{i j r}$ é distribuído como $N\left(0, \sigma_{e}^{2}\right)$.

Com base nas suposições, são construídas, de modo adequado, as funçōes de verossimilhança que combinadas com as distribuiçōes a priori nàoinformativas para os parâmetros dos modelos geram-se densidades a posteriori, com a restrição de que os componentes da variância não assumem valores negativos.

A partir da densidade conjunta a posteriori dos paràmetros do modelo. $\left(\boldsymbol{\theta}, \sigma_{e}^{2}, \sigma_{g}^{2}, \sigma_{b}^{2}\right)$, isto é, $p\left(\boldsymbol{\theta}, \sigma_{e}^{2}, \sigma_{g}^{2}, \sigma_{b}^{2} \mid \boldsymbol{y}\right)$, são determinadas densidades marginais para o vetor de paràmetros de locação $\boldsymbol{\theta}=\left(\theta_{1}, \ldots, \theta_{K}\right)^{\prime}, p(\boldsymbol{\theta} \mid \boldsymbol{y})$, e para combinações lineares destes, isto é, $\boldsymbol{\phi}=\mathrm{D} \boldsymbol{\theta}=\left(\phi_{1}, \ldots, \phi_{(K-1)}\right)^{\prime}, p(\boldsymbol{\phi} \mid \boldsymbol{y})$ de modo conveniente, para tornar possível a verificação de evidèncias de contrastes de interesse do pesquisador, bem como, estudar o comportamento da variável resposta em relação aos níveis do fator tratamento através de polinòmios ortogonais. Também é determinada a densidade conjunta a posteriori dos componentes de variância, $p\left(\sigma_{e}^{2}, \sigma_{g}^{2}, \sigma_{b}^{2} \mid \boldsymbol{y}\right)$, bem como. aproximaçòes para as densidades marginais a posteriori destes componentes, $p\left(\sigma_{e}^{2} \mid \boldsymbol{y}\right) \cdot p\left(\sigma_{g}^{2} \mid \boldsymbol{y}\right)$ e $p\left(\sigma_{b}^{2} \mid \boldsymbol{y}\right)$.

Um grupo de très experimentos de adubação com micronutrientes em cana-de-açúcar foi usado para ilustrar a metodologia Bayesiana e uma análise clássica foi também realizada para se comparar as metodologias. 


\title{
A BAYESIAN ANALYSIS FOR A SERIES OF EXPERIMENTS IN COMPLETELY RANDOMIZED DESIGN
}

\author{
Author: João Gil de Luna \\ Adviser: Prof. Dr. Humberto de Campos
}

\section{SUMMARY}

Statistical analysis of a series of experiments is very common in agricultural experimentation. In this situation, the researcher is often interested not only in the determination of effects for the individual experiments given by particular choices of environments (agricultural year, seasons of the year, places, types of soils, etc.) but also in the general effects of treatments, environments and interation.

The present study develops a Bayesian strategy to analyse a series of experiments in completely randomized design when the researcher is interested in getting generic conclusions about the treatment factor (a fixed factor) and in getting the components of variance for the experiment factor and for the interaction treatments $\times$ experiments (random effect).

For the individual analysis it is considered the model $y_{i r}=\theta_{i}+\epsilon_{i r}$, where $y_{i r}$ is the $r$-th observation for the plot under the treatment $i, \theta_{i}$ is the mean effect of treatment $i$ and $\epsilon_{i r}$ is the error with $N\left(0, \sigma^{2}\right)$ distribution. A mixed model is considered for the analysis of series of experiments, that is, $y_{i j r}=\theta_{i}+\beta_{j}+\gamma_{i j}+\epsilon_{i j r}$, where $y_{i j r}$ is the observation for the $r$-th plot which received the treatment $i$, in the $j$-th experiment; $\beta_{j}$ is the $j$-th experiment effect; $\gamma_{i j}$ is the interaction effect and $\epsilon_{i j r}$ is the error with $N\left(0, \sigma_{e}^{2}\right)$ distribution.

The likelihood functions are developed following the assumptions 
and combined with the non-informative priori density for the model parameters generating the posteriori with the restriction that components of variance are nonnegative. Using the posteriori joint density of the parameters, $p\left(\boldsymbol{\theta}, \sigma_{e}^{2}, \sigma_{g}^{2}, \sigma_{b}^{2} \mid \boldsymbol{y}\right)$, the marginal density are developed for the parameter vector $\boldsymbol{\theta}=\left(\theta_{1}, \cdots, \theta_{K}\right)^{\prime}, p(\boldsymbol{\theta} \mid \boldsymbol{y})$, and for the linear combinations of them. that is, $\phi=\left(\phi_{1}, \cdots, \phi_{(K-1)}\right)^{\prime}, p(\phi \mid \boldsymbol{y})$. This is used to test contrasts of experimenter's interest and to study the relationship between the response variable and the levels of the treatment factor using orthogonal polinomyals. It is also developed the posteriori joint density for the components of variance, $p\left(\sigma_{e}^{2}, \sigma_{g}^{2}, \sigma_{b}^{2} \mid \boldsymbol{y}\right)$, as well as the approximations for the posterior marginal densities, $p\left(\sigma_{e}^{2} \mid \boldsymbol{y}\right), p\left(\sigma_{g}^{2} \mid \boldsymbol{y}\right)$ e $p\left(\sigma_{b}^{2} \mid \boldsymbol{y}\right)$.

The Bayesian methodology was illustrated with a series of three experiments using micronutrients in sugarcane. The results were compared with classical analysis. 


\section{INTRODUÇÃO}

Em experimentação agronômica é muito freqüente a estruturação de análises estatísticas de grupos de experimentos. Vários são os motivos que levam a essa prática. O desempenho da produção das plantas, por exemplo, depende do genótipo, do ambiente e da interação genótipo/ambiente. Genótipos e alguns outros fatores relativos a manejos, tais como, quantidade de adubo no solo, controle de pragas, densidade de plantio, preparo do solo, etc, podem ser controlados pelo pesquisador. Fatores ambientais, tais como, velocidade do vento, insolação, precipitação pluvial, taxas de nutrientes no solo, etc, além de serem, em geral, incontroláveis, podem modificar as produções das plantas de um determinado local ou época. Dada a impossibilidade de controle desses fatores, eles são considerados pelo pesquisador como fixados. Desse modo, com um único experimento, o pesquisador poderá obter conclusões apenas para os fatores controláveis, o que não ocorre com os fatores ambientais, que estão além do seu controle.

Os efeitos dos fatores ambientais incontroláveis no desempenho da produção das plantas cultivadas, são tão importantes quanto os fatores controláveis, e a avaliação quantitativa de seus efeitos é de fundamental importància, porque se espera que esses efeitos podem mudar com o local ou a época do cultivo. Além disso, o efeito do ambiente sobre a resposta das plantas aos tratamentos, poderá também ser avaliado. Em pesquisa agronômica, a maneira usada para medir o efeito do ambiente sobre a produção das plantas é através da repetição do experimento em vários locais ou em várias épocas ou ambos.

Um outro objetivo a ser alcançado com a prática da análise de 
grupos de experimentos, associa-se ao interesse do pesquisador em inferir resultados da pesquisa para condições gerais de uma grande propriedade ou região. Nesse caso, ele deve considerar uma série de ensaios instalados em diferentes locais e em diferentes épocas e anos. Essa situação ocorre em programas de melhoramento genético de plantas onde o critério de lançamento de uma nova variedade comercial se baseia no comportamento relativo das cultivares em diversos locais e anos.

Observa-se, portanto, que a análise conjunta de experimentos procura fazer inferência sobre os efeitos do fator tratamento aplicados aos ensaios individuais e sobre o fator experimento que poderá estar representando na análise conjunta, épocas, estações do ano, tipos de solo, locais, etc, ou simplesmente repetições do ensaio.

É evidente que conclusões para cada situação experimental poderão ser obtidas, analisando-se individualmente cada ensaio e conclusões mais gerais, analisando-se o grupo conjuntamente.

$\mathrm{Na}$ ocasião do planejamento da pesquisa, algumas recomendações técnicas deverão ser seguidas, de modo a facilitar ou tornar possível, posteriormente, a verificação das hipóteses de interesse. Sob este aspecto, recomenda-se o balanceamento intra e inter ensaios e que o delineamento experimental seja o mesmo em todos eles. A não observância dessas características poderá levar a problemas de não estimabilidade das funções paramétricas que representam as hipóteses de interesse e, portanto, estas não poderão ser testadas.

Na literatura, do ponto de vista da inferência clássica, são encontrados vários trabalhos que se propõem a fornecer subsídios para análise de grupos de experimentos com as características recomendadas anteriormente e também para grupos, onde apenas alguns tratamentos são comuns a todos os ensaios. Porém, do ponto de vista Bayesiano, não se tem notícia de trabalhos dedicados especificamente a esse assunto, de tão grande importância na pesquisa.

A análise estatística de experimentos via Inferência Bayesiana, apresenta algumas vantagens importantes sobre a teoria clássica. Dentre elas podem 
ser citadas:

- É possível incorporar na análise, informações a priori acerca dos tratamentos, obtidas de experimentos anteriores e as inferências serão feitas com base na densidade a posteriori, a qual combina informações do passado e do presente sobre os paràmetros de interesse;

- Facilidade de interpretação dos resultados ao enfocar questões práticas onde as comparações múltiplas não apresentam resultados satisfatórios;

- Possibilidade de avaliar a probabilidade a posteriori de que qualquer genótipo (ou tratamento) em particular tenha melhor produção ou que qualquer grupo de genótipos (ou tratamentos) seja melhor. Estas questões são de grande importância em programas de melhoramento onde se pretende selecionar o melhor genótipo ou selecionar o melhor subgrupo de um grande conjunto de genótipos.

Tendo em vista essas vantagens, pretende-se neste trabalho apresentar uma solução Bayesiana para a análise estatística de um grupo de experimentos completamente aleatorizados, onde o interesse do pesquisador é fazer inferência sobre os efeitos médios dos tratamentos, considerados fixos, sobre os efeitos de experimentos, considerados aleatórios e, ainda, avaliar o comportamento da variável resposta em relação aos tratamentos dentro dos experimentos.

O interesse no estudo deste caso, deve-se à grande frequência de sua ocorrência na prática e uma análise estatística mais elaborada trará, sem dúvida, contribuições importantes à pesquisa científica onde essa situação for encontrada.

Além do objetivo principal já citado acima, pretende-se alcançar, neste trabalho, os seguintes objetivos específicos:

- Apresentar, as deduções teóricas, de modo a facilitar o desenvolvimento de outras análises empregando a metodologia Bayesiana; 
- Definir apropriadamente os modelos, bem como, discutir as pressuposições usuais acerca de seus componentes, desenvolvendo-se metodologias para verificação de hipóteses sobre elas;

- Apresentar uma discussão relativa à incorporação de informação a priori para os paràmetros envolvidos no modelo, de modo a tornar clara a escolha desta, para o modelo em questão;

- Propor uma forma adequada de se incorporar a informação presente, através da verossimilhança, para determinação da densidade a posteriori;

- Discutir as propriedades relativas aos paràmetros sobre os quais se deseja fazer inferència, como também, relativas às combinaçōes lineares deles;

- Realizar as análises estatísticas de um grupo de experimentos inteiramente casualizados, empregando as duas metodologias: a Clássica e a Bayesiana, discutindo-se os resultados obtidos. paralelamente. 


\section{REVISÃO DE LITERATURA}

\subsection{Análise de Grupo de Experimentos}

Os primeiros trabalhos contemplando a análise de grupo de experimentos, são devidos a Cochran (1937) e Yates \& Cochran (1938). A técnica tornou-se possível, após BARTLETT (1937) ter proposto um teste para verificar a homogeneidade das variâncias de populações normais, a qual é uma condição necessária ao agrupamento dos ensaios numa análise de variância. Este assunto, é também abordado em vários livros textos que tratam de delineamentos experimentais. Dentre eles, Cochran \& Cox (1957), Kempthorne (1973), Gomez \& Gomez (1984), Campos (1984), Gomes (1985).

A análise de grupo de experimentos sugerida por Cochran \& Cox (1957), considera o caso em que os ensaios individuais são instalados em blocos completos ao acaso com $v$ tratamentos, $\ell$ locais e $a$ anos, sendo que os $\ell$ locais são comuns a todos os anos. O modelo utilizado para análise, leva em conta apenas as médias dos tratamentos em cada local.

Em situação semelhante, KEMPTHORNE (1973) sugere um modelo onde todas as observações de cada experimento individual são consideradas na análise conjunta.

Gomez \& GoMez (1984) apresentam a análise conjunta como uma técnica utilizada em experimentação agronômica para controlar os efeitos de fatores que fogem ao controle do pesquisador. Nesse sentido, os autores consideram como fatores: local, ano, tipo de solo, estação do ano, etc., além dos tratamentos considerados em cada ensaio. 
CAmpos (1984), dentre outros, apresenta uma análise conjunta de experimentos em blocos ao acaso com apenas alguns tratamentos comuns a todos os ensaios. É, sem dúvida, uma sugestão bastante aplicável em programas de meIhoramento genético de plantas cultiváveis.

GOMES (1985) apresenta as análises de grupos de experimentos inteiramente ao acaso e de experimentos em blocos ao acaso e, ao contrário de GOMEz \& GOMEz (1984), o autor considera local, ano, tipo de solo, estação do ano. etc.. indistintamente, como ensaios e não como fontes de variação nas quais se tem interesse em tirar conclusões sobre os efeitos delas.

A análise de grupo de experimentos pressupõe, além de normalidade para os erros experimentais e aditividade dos componentes no modelo, a homogeneidade das variàncias dos erros residuais dos ensaios envolvidos. Nesse sentido, COCHRAN \& COX (1957) e KEMPTHORNE (1973) afirmam que o teste proposto por BARTLETT (1937) pode ser empregado para verificar essa hipótese. Contrariamente, Box (1953), em estudo sobre não-normalidade e teste sobre variâncias, conclui que o teste de Bartlett é tão sensível à falta de normalidade que não deve ser considerado. No entanto, segundo Box (1954), se em todos os experimentos considerados no grupo. os tratamentos forem igualmente repetidos. e a relação entre o maior e o menor quadrado médio residual não for maior que quatro, a análise poderá ser realizada sem grandes prejuízos para a validade do teste de Bartlett. Recomenda ainda, que se essa relação for superior a quatro, os experimentos devem ser agrupados em sub-grupos cujos quadrados médios residuais sejam homogèneos.

Uma sugestão proposta por CoCHRAN (1954), bastante utilizada na prática. e que é recomendada por Gomes (1985), admite o agrupamento de ensaios onde a relação entre os quadrados médios residuais é superior a quatro. Neste caso, procede-se a um ajuste dos graus de liberdade.

Observa-se, portanto, que a análise de grupo de experimentos é $11 \mathrm{~m}$ tema bastante estudado do ponto de vista da inferència clássica. Porém, ainda não é um assunto completamente esgotado devido a diversidade da natureza dos 
problemas práticos. $\mathrm{E}$, deste modo, novas teorias poderão ser implementadas para solucionar problemas específicos.

Nos últimos anos, a teoria Bayesiana tem sido bastante difundida nos meios científicos das diversas áreas do conhecimento. Porém na literatura estatística não foi encontrado nenhum artigo que tratasse a análise de grupo de experimentos fundamentada nessa teoria.

\subsection{A Natureza do Método Bayesiano}

Conforme Box \& Tiao (1973), a natureza do método Bayesiano compreende, dentre outros, os seguintes aspectos:

\subsubsection{O Teorema de Bayes}

Supōe-se que $\boldsymbol{y}^{\prime}=\left(y_{1}, \ldots, y_{n}\right)$ é um vetor de $n$ observações cuja distribuição de probabilidade $p(\boldsymbol{y} \mid \boldsymbol{\theta})$ depende dos valores de $k$ parâmetros $\boldsymbol{\theta}^{\prime}=$ $\left(\theta_{1}, \ldots, \theta_{k}\right)$. Supōe-se também, que $\boldsymbol{\theta}$ tem uma distribuição de probabilidade $p(\boldsymbol{\theta})$. Então.

$$
p(\boldsymbol{y}\{\boldsymbol{\theta}) p(\boldsymbol{\theta})=p(\boldsymbol{y}, \boldsymbol{\theta})=p(\boldsymbol{\theta} \mid \boldsymbol{y}) p(\boldsymbol{y})
$$

Dadas as observações $\boldsymbol{y}$, a distribuição condicional de $\boldsymbol{\theta}$ é

$$
p(\boldsymbol{\theta} \mid \boldsymbol{y})=\frac{p(\boldsymbol{y} \mid \boldsymbol{\theta}) p(\boldsymbol{\theta})}{p(\boldsymbol{y})}
$$

Na expressão (2) $p(\boldsymbol{y})=W^{-1}$ é chamada de constante normalizadora e é dada por

$$
p(\boldsymbol{y})=E[p(\boldsymbol{y} \mid \boldsymbol{\theta})]=W^{-1}=\left\{\begin{array}{l}
\int_{\boldsymbol{\theta}} p(\boldsymbol{y} \mid \boldsymbol{\theta}) p(\boldsymbol{\theta}) d \boldsymbol{\theta}, \quad \boldsymbol{\theta} \text { continuo } \\
\sum p(\boldsymbol{y} \mid \boldsymbol{\theta}) p(\boldsymbol{\theta}), \quad \boldsymbol{\theta} \text { discreto, }
\end{array}\right.
$$

onde a integral ou soma é tomada sobre a amplitude admissível de $\boldsymbol{\theta}$, e onde $E[f(\boldsymbol{\theta})]$ 
é a esperança matemática de $f(\boldsymbol{\theta})$ relativa à distribuição $p(\boldsymbol{\theta})$. Dessa forma, pode-se escrever (2), alternativamente, como

$$
p(\boldsymbol{\theta} \mid \boldsymbol{y})=W p(\boldsymbol{y} \mid \boldsymbol{\theta}) p(\boldsymbol{\theta})
$$

A expressão (2) ou equivalente em (4), é normalmente conhecida como o Teorema de Bayes. Nestas expressões, $p(\boldsymbol{\theta})$, diz o que é conhecido sobre $\boldsymbol{\theta}$ sem o conhecimento dos dados, e é chamada de distribuição a priori de $\boldsymbol{\theta}$, ou a distribuição de $\boldsymbol{\theta}$ a priori. Correspondentemente, $p(\boldsymbol{\theta} \mid \boldsymbol{y})$ diz o que é conhecido sobre $\theta$ dado o conhecimento das observações, e é chamada distribuição a posteriori de $\boldsymbol{\theta} . p(\boldsymbol{\theta} \mid \boldsymbol{y})$, encerra todo conhecimento, do "passado" e do "presente", acerca de $\boldsymbol{\theta}$. É com base nesta distribuição que os Estatísticos Bayesianos fazem todas as inferèncias sobre o vetor de paràmetros $\boldsymbol{\theta}$. É importante notar que uma vez conhecida a densidade a posteriori de $\boldsymbol{\theta}$, pode-se obter "tudo" sobre ele, a saber, média, moda. mediana, variància, intervalos de credibilidades, fazer testes de hipóteses, etc. A quantidade $W$ é simplesmente uma constante normalizadora para garantir que a densidade a posteriori, $p(\boldsymbol{\theta} \mid \boldsymbol{y})$, seja uma função de densidade própria.

\subsubsection{O Teorema de Bayes e a Função de Verossimilhança}

Dadas as observações $\boldsymbol{y}, p(\boldsymbol{y} \mid \boldsymbol{\theta})$ em (4) pode ser considerada como uma função não de $\boldsymbol{y}$, mas sim de $\boldsymbol{\theta}$. Quando assim for considerada, ela é chamada de função de verossimilhança de $\boldsymbol{\theta}$ dado $\boldsymbol{y}$ e pode ser escrita como $\ell(\boldsymbol{\theta} \mid \boldsymbol{y})$. Assim. pode-se escrever a fórmula de Bayes como

$$
p(\boldsymbol{\theta} \mid \boldsymbol{y}) \propto \ell(\boldsymbol{\theta} \mid \boldsymbol{y}) p(\boldsymbol{\theta})
$$

Em outras palavras, o teorema de Bayes diz que a distribuição de probabilidade de $\boldsymbol{\theta}$ posterior aos dados $\boldsymbol{y}$ é proporcional ao produto da verossimilhança pela distribuição a priori de $\boldsymbol{\theta}$.

A função de verossimilhança $\ell(\boldsymbol{\theta} \mid \boldsymbol{y})$ representa na fórmula de Bayes, a funçào através da qual os dados modificam o conhecimento a priori sobre $\boldsymbol{\theta}$. Ela 
pode ser compreendida como representante da informação sobre $\boldsymbol{\theta}$ proveniente dos dados.

\subsubsection{A Natureza Sequencial do Teorema de Bayes}

A expressão (5) é uma formulação matemática recorrente de como o conhecimento prévio pode ser combinado com os novos conhecimentos. Sem dúvida, o teorema de Bayes permite que contínuas informações acerca do conjunto de paràmetros $\boldsymbol{\theta}$ sejam incorporadas a $p(\boldsymbol{\theta} \mid \boldsymbol{y})$ à medida que mais observações forem tomadas. Desse modo, tem-se que, para uma amostra inicial de observações $\boldsymbol{y}_{1}$, a fórmula de Bayes será dada por

$$
p\left(\boldsymbol{\theta} \mid \boldsymbol{y}_{1}\right) \propto p(\boldsymbol{\theta}) \ell\left(\boldsymbol{\theta} \mid \boldsymbol{y}_{1}\right)
$$

Se for obtida uma segunda amostra de observações $\boldsymbol{y}_{2}$, distribuída independentemente da primeira amostra $\boldsymbol{y}_{1}$, então

$$
p\left(\boldsymbol{\theta} \mid \boldsymbol{y}_{2}, \boldsymbol{y}_{1}\right) \propto p(\boldsymbol{\theta}) \ell\left(\boldsymbol{\theta} \mid \boldsymbol{y}_{1}\right) \ell\left(\boldsymbol{\theta} \mid \boldsymbol{y}_{2}\right)=p\left(\boldsymbol{\theta} \mid \boldsymbol{y}_{1}\right) \cdot \ell\left(\boldsymbol{\theta} \mid \boldsymbol{y}_{2}\right)
$$

A expressão dada em (7) é exatamente a mesma de (6), exceto que $p\left(\boldsymbol{\theta} \mid \boldsymbol{y}_{1}\right)$, a distribuição a posteriori de $\boldsymbol{\theta}$ dado $\boldsymbol{y}_{1}$, faz o papel da distribuição a priori para a segunda amostra. Obviamente, esse processo pode ser repetido várias vezes. Em particular, para $n$ observações independentes, a distribuição a posteriori pode, se desejada, ser calculada após cada nova observação, desde que no $m$-ésimo estágio a verossimilhança associada à $m$-ésima observação seja combinada com a distribuição a posteriori de $\boldsymbol{\theta}$ após a $(m-1)$-ésima observação para gerar a nova distribuição $a$ posteriori, ou seja,

$$
p\left(\boldsymbol{\theta}\left|y_{1}, \ldots, y_{m}\right\rangle \propto p\left(\boldsymbol{\theta} \mid y_{1}, \ldots, y_{m-1}\right) . \ell\left(\boldsymbol{\theta} \mid y_{m}\right), \quad m=2, \ldots, n\right.
$$

onde $p\left(\boldsymbol{\theta} \mid \boldsymbol{y}_{1}\right)$ é de acordo como já definida em (6) 
Dessa forma, o teorema de Bayes descreve, de maneira fundamental, o processo de aprender por experiència, e mostra como o conhecimento sobre o estado de natureza representado por $\boldsymbol{\theta}$ é modificado à medida que novos dados são avaliados.

\subsection{Inferência Bayesiana Básica}

Em livro texto com primeira edição em 1939, JEFFREYS (1961) sintetiza e amplia os conceitos relativos à inferència indutiva e utiliza o teorema de Bayes para formalizar diversos empregos da estatística matemática sob esse novo enfoque. $\mathrm{O}$ autor é considerado como o precursor de métodos alternativos à estatística clássica.

Uma importante contribuição para inferència Bayesiana é, sem dúvida. o trabalho de BARNARD (1958) o qual apresenta uma edição mais recente dos trabalhos de Thomas Bayes, de 1763, onde é desenvolvida uma série de proposiçōes relativas à adição e multiplicação de probabilidades e conclui com o teorema da probabilidade inversa.

LindLey (1965) dedica livro texto ao enfoque Bayesiano, sendo que a primeira parte aborda conceitos da teoria das probabilidades, com ènfase especial à interpretação do teorema de Bayes e suas aplicações. A segunda parte é dedicada à teoria Bayesiana com aplicações, com resultados comparados com aqueles da inferència clássica.

ZACKS (1971) apresenta uma abordagem mais profunda sobre os métodos da inferència estatística, incluindo no seu conteúdo dedicaçòes ao processo minimax e ao método Bayesiano.

LINDLEY (1972) apresenta uma monografia abordando os mais variados aspectos da inferència Bayesiana e compara a eficiència deste enfoque com a teoria clássica. Apresenta também as conquistas e limitaçōes com base nos trabalhos até então publicados. É um artigo de grande importância, pois, sintetiza as con quistas do método Bayesiano até aquele momento e apresenta, no seu final, 
indicação bibliográfica com 270 referências.

Os fundamentos do método Bayesiano, nos seus aspectos gerais, e os elementos necessários a um estudo inicial, são encontrados nos trabalhos de Stuart \& Ord (1961), Box \& Tiao (1973,1992), Mood \& Graybill (1974), Bakman \& Neto (1980), Simões (1981), Pereira \& Viana (1982), Degroot (1988), HogG \& CRAIG (1989), dentre outros.

Além dos trabalhos citados no parágrafo anterior, ao leitor que desejar aprofundar seus conhecimentos relativos aos métodos Bayesianos, é recomendável a leitura dos livros texto, publicados mais recentemente, com autoria de LEE (1992), O'HAGAN (1994) e BERNARDo \& SMith (1994). Estes três trabalhos apresentam a teoria Bayesiana com maior profundidade do que os anteriores.

\subsection{A Informação a Priori}

Com relação à distribuição a priori $p(\boldsymbol{\theta})$, ela deverá representar o conhecimento (ou desconhecimento) do Pesquisador/Estatístico a respeito do comportamento probabilístico de $\boldsymbol{\theta}$. Neste sentido, LEANDRo (1994), apresenta très situaçōes de escolha da distribuição a priori para o(s) parâmetro(s), as quais são descritas a seguir.

Distribuições Ajustadas às Frequências Relativas: São baseadas em quantidades significativas de evidèncias, onde distribuições de frequências, de valores de $\boldsymbol{\theta}$ observados no passado, sejam determinadas. A partir destas, pode-se escolher uma distribuição a priori para o vetor de parâmetros $\boldsymbol{\theta}$ apropriadamente, sendo possível selecionar um elemento da família de conjugadas naturais. Isto é, uma priori para $\boldsymbol{\theta}$ que, ao ser combinada com a verossimilhança, gera uma densidade a posteriori para $\boldsymbol{\theta}$ que pertence à mesma família de densidades de probabilidade.

Distribuições Ajustadas a Expectativas Subjetivas: São empregadas em situaçōes onde não se tem nenhuma base objetiva sólida para a escolha da 
distribuição a priori de $\boldsymbol{\theta}$. Neste caso, $p(\boldsymbol{\theta}\rangle$ representará apenas as expectativas subjetivas do pesquisador. Sendo assim, cabe ao Estatístico esclarecer ao pesquisador as implicações de sua escolha na distribuição a posteriori.

Distribuições Objetivas: Este tipo de priori é admitida quando o Pesquisador acha que o seu conhecimento sobre $\boldsymbol{\theta}$ não é suficiente para avaliá-lo, ou ainda, quando ele deseja expressar através da priori sua indiferença ou ignorância a respeito do comportamento probabilístico do parâmetro $\boldsymbol{\theta}$. Neste caso, deve ser admitida priori's não-informativas.

RAIFFA \& Schlaiffer (1961), em livro texto, apresentam uma forma especial de distribuição a priori para representar a informação que se obtém mediante experimentos anteriores. É chamada de priori conjugada e tem a característica de que, ao ser combinada com a verossimilhança, gera uma densidade $a$ posteriori que pertence à mesma família de distribuições.

TiaO \& Zellner (1964) citam vários trabalhos que empregaram o teorema de Bayes na inferència estatística e destacam os trabalhos de JEFFREYS (1961), Raiffa \& Schlaiffer (1961), Box \& Tiao $(1962,1964)$. Os autores afirmam, ainda, que uma das vantagens da inferència Bayesiana é que conhecimentos a priori sobre os parâmetros de interesse podem ser combinados com informações obtidas do experimento. Estes conhecimentos podem surgir de considerações teóricas gerais assim como de resultados prévios ou experimentos em andamento, e são usualmente, um componente importante para o pesquisador compreender melhor o que está em estudo. O trabalho ilustra como os conhecimentos a priori podem ser utilizados em conjunto com as informações amostrais ao fazer inferência sobre os paràmetros de um modelo de regressão linear simples. Em particular, o trabalho contempla o uso da informação de uma amostra como o conhecimento a priori na análise de uma amostra subsequente.

OWEN (1970) trabalha com um modelo de blocos casualizados onde o interesse é fazer inferència sobre os efeitos médios dos tratamentos. Os efeitos dos blocos foram considerados na análise como parâmetros de perturbação. Assumindo 
para os erros uma distribuição esfericamente normal multivariada $N_{n}\left(0, \sigma^{2} I_{n}\right)$ e a distribuição a priori para os paràmetros do modelo aproximadamente independentes e localmente uniformes, o autor determina a função de densidade a posteriori para os efeitos médios dos tratamentos e as inferências foram feitas a partir dela. Com uma função de perda quadrática, um algoritmo foi determinado para a alocação ótima dos tratamentos sobre uma amostra particular.

Mais tarde, ZELLNER (1971) apresenta a importância das regras de JEFFREYS (1961) para inferência indutiva e discute suas implicações no processo indutivo. Além disso, dedica todo conteúdo do livro aos modelos econométricos, com bastante aplicações.

Box \& Tiao (1973) também discutem as idéias de JefFreys (1961), sobre a distribuição a priori para representar o estado de ausência de informação (ou estado de ignorància) a respeito do comportamento probabilístico dos paràmetros, o estudo abrange os casos uniparamétrico e multiparamétrico. É, talvez, a discussão mais difundida sobre priori não-informativa. Trabalhando com esse tipo de priori para os paràmetros, os autores desenvolvem a teoria Bayesiana para o modelo com um fator, abordando duas situações: (a) o fator é de efeito fixo; (b) o fator é de efeito aleatório. Além disso, desenvolvem a teoria Bayesiana para um modelo hierárquico de efeito aleatório com três componentes de variància.

Considerando uma priori informativa Normal-Gama para os paràmetros do modelo, Broemeling (1985) apresenta em seu livro texto o desenvolvimento teórico para análises estatísticas, do ponto de vista Bayesiano, para os modelos inteiramente casualizado com um fator, blocos ao acaso, modelos para experimentos inteiramente casualizado com uma covariável e modelos de regressão.

YE \& BERGER (1993) estudando um modelo de regressão exponencial, afirmam que há dificuldades para se fazer inferência acerca dos parâmetros da regressão no enfoque clássico. Tais dificuldades também são enfrentadas na aproximação Bayesiana com priori não informativa. Uma priori de referência é adotada no artigo para produzir inferèncias satisfatórias. Estimação e regiões de credibilidade 
são determinadas a partir de um exemplo especial.

Clarke \& Wasserman (1993) abordam o conflito entre priori's que são não-informativas para os paràmetros de interesse versus priori's que são nãoinformativas para todos os paràmetros enrolvidos no modelo. A pesquisa leva os autores a maximizar uma funçào que tinha dois termos: uma aproximação assintótica para a distància de Kullback-Leibler esperada padronizada entre priori marginal e posteriori marginal dos paràmetros de interesse, e, um termo penalizador medindo a distància da priori a priori de Jeffreys. Uma constante positiva multiplicando o segundo termo determina o termo ausente entre nào informação para os paràmetros de interesse e a nào informação para todos os paràmetros do modelo. Os autores constataram que conforme aumenta a constante, a priori aproxima-se da priori de Jeffreys e quando a constante tende para zero a priori torna-se degenerada. Exceto para alguns casos especiais, essa priori não tem uma forma fechada, mas apresenta um algoritmo bastante simples para determiná-la.

LisEo (1993) afirma que o problema de paràmetros nuisance é tratado de diferentes maneiras. As técnicas de verossimilhança padrào, tais como perfil de verossimilhança e suas modificaçōes, são comparadas pelo autor com a análise Bayesiana baseada em priori de referència. $O$ autor apresenta exemplos numéricos para ilustrar o que acontece em problemas multiparamétricos e discute as rant agens e desvantagens das técnicas Bayesiana e verossimilhança.

BESAG \& Higdon (1993) trabalhando com experimentos agrícolas com delineamentos espaciais, assumem que os dados são provenientes de populaçòes normais, pressupòem priori informativa normal para os efeitos dos tratamentos e fertilidade das unidades experimentais e priori informativa gama para as variàncias associadas aos efeitos do modelo.

Nos últimos anos, as pesquisas em inferència Bayesiana têm dado bastante atenção ao estudo de priori's robustas. Para mostrar a importància e dimensão dessa tendència, BERGER (1994), editou no Journal of Statistical Planning and Inference. em edição especial, 16 importantes trabalhos sobre o assunto. 


\subsection{Modelos Lineares Bayesianos}

BOX \& TIAO (1965) apresentam a teoria Bayesiana para analisar um experimento associado a um modelo linear com um fator de efeito fixo admitindose que os dados do ensaio são provenientes de populaçoes normais com priori não informativa para $\left(\boldsymbol{\theta}, \sigma^{2}\right)$, onde $\boldsymbol{\theta}$ e $\log \sigma$ são aproximadamente independentes e localmente uniformes. Ainda, para esse mesmo modelo, TIAO \& TAN (1965), estudaram o caso onde o efeito do fator foi considerado aleatório, os dados também provenientes de populações normais e a distribuição a priori para $\left(\mu, \sigma_{a}^{2}, \sigma_{e}^{2}\right)$ não-informativa com $\left(\mu, \log \sigma_{a}, \log \sigma_{e}\right)$ aproximadamente independentes e localmente uniformes. Com essas suposiçòes eles desenvolvem toda teoria necessária para se fazer inferência sobre os componentes de variância e algumas relaçōes entre eles. Posteriormente, Box \& TIAO (1971), além dos dois artigos anteriormente citados, discutem modelos para experimentos hierárquicos com fatores aleatórios, para experimentos com dois fatores aleatórios cruzados, modelo de regressão e o modelo linear geral. Ainda, Draper \& Guttman (1966), Box \& Tiao (1968), Portnoy (1971), dentre outros, apresentaram sugestões teóricas, do ponto de vista Bayesiano, para solucionar problemas relativos ao modelo associado a um ensaio completamente aleatorizado com um fator.

TiaO \& DraPer (1968) apresentam soluções analíticas para as análises estatísticas do ponto de vista Bayesiano para experimentos com dois fatores. Nesse sentido, os autores consideraram os modelos associados aos ensaios em blocos completos ao acaso, como também, em blocos incompletos. Do mesmo modo, BRoemeling (1985) considera em seu livro texto a mesma metodologia para solução de iguais problemas. Outros trabalhos relacionados com o emprego da inferència Bayesiana na experimentação agrícola que poderão ser recomendados, são devidos a Besag \& Higdon (1993) e LaUd \& Ibrahin(1994). 


\subsection{Testes Bayesianos de Homogeneidade de Variâncias}

BoX \& TiAO (1964) estudam os fundamentos teóricos Bayesianos para o teste de homogeneidade das variàncias de populações normais, proposto por BARTLETT (1937). Os autores tornam esse artigo, parte integrante de um livro texto em 1973, onde apresentam, além da teoria, um exemplo prático para ilustrar o método.

Dada a sensibilidade do teste de Bartlett à falta de normalidade das populaçōes amostradas, SAmiUdDIN (1976) propōe um teste Bayesiano mais adequado a situaçōes de não-normalidade. Nesse trabalho, o autor expõe os fundamentos teóricos do teste e apresenta exemplos ilustrativos, comparando os resultados obtidos pelo teste proposto com as duas versōes do teste de Bartlett. 


\section{DESENVOLVIMENTO TEÓRICO}

\subsection{Generalidades}

Na ocasião do planejamento de um ensaio, o pesquisador deverá ter ciència das hipóteses básicas a serem verificadas. Esta preocupação é de fundamental importància no que se refere a considerar se os fatores envolvidos nos ensaios serão de efeitos fixos ou de efeitos aleatórios. Quando um fator é considerado de efeito fixo, em geral, o pesquisador estará interessado em construir densidades a posteriori para os efeitos médios dos $K$ tratamentos $\left(\theta_{1}, \ldots, \theta_{K}\right)$ ou para combinações lineares desses paràmetros e testar hipóteses sobre eles ou sobre combinações lineares deles. Caso um fator seja considerado de efeito aleatório, o interesse do pesquisador estará mais roltado a determinação de densidades a posteriori para os componentes de variância, relações ou combinações lineares deles. Finalmente, se o modelo estatístico associado ao ensaio envolver fatores fixos e aleatórios, estes devem ser tratados adequadamente conforme a natureza de cada um.

Neste trabalho, pretende-se apresentar uma análise Bayesiana para um grupo de $J$ experimentos inteiramente casualizados, cada um com $K$ tratamentos repetidos $R$ vezes, onde o fator tratamento será considerado de efeito fixo, o fator experimento e a interação tratamento $\times$ experimento serão considerados de efeitos aleatórios. Além disso, recomenda-se que as análises estatísticas sejam realizadas quando as varâncias dos erros experimentais dos $J$ experimentos envolvidos, são homogèneas. Caso isso não ocorra, deve-se agrupá-los em subgrupos homogèneos, conforme recomendação de Box (1953).

Una análise conjunta de experimentos é precedida das análises in- 
dividuais de cada ensaio e este procedimento será também adotado, neste trabalho.

\subsection{As Análises Individuais}

\subsubsection{O Modelo Estatístico}

O modelo estatístico associado a um experimento inteiramente casualizado, com um fator em $K$ níveis, repetidos $R$ vezes, é dado por

$$
y_{i r}=\mu+\alpha_{i}+e_{i r}, \quad i=1, \ldots, K \quad \text { e } \quad r=1, \ldots, R,
$$

onde $y_{i r}$ é a $r$-ésima observação da unidade experimental que recebeu o tratamento $i, \mu$ é a média geral, $\alpha_{i}$ é o efeito do $i$-ésimo tratamento, aqui considerado fixo, e $e_{i r}$ é o erro experimental associado à observação $y_{i r}$.

Alternativamente, pode-se trabalhar com o modelo de médias das caselas, o qual é dado por

$$
y_{i r}=\theta_{i}+e_{i r}, \quad i=1, \ldots, K \quad \text { e } \quad r=1, \ldots, R,
$$

onde $\theta_{i}$ corresponde à resposta média do tratamento $i$, e os demais termos no modelo, conforme já definidos.

Neste trabalho preferiu-se o modelo (10) por ser matematicamente mais conveniente. Sua forma matricial é como segue

$$
\boldsymbol{y}=X \boldsymbol{\theta}+\boldsymbol{\varepsilon}
$$

onde $y$ é um vetor de realizações de variáveis aleatórias com dimensões $n \times 1, n=$ $K R ; X$ é uma matriz de constantes (ou a matriz de planejamento) com dimensões $n \times K ; \boldsymbol{\theta}=\left(\theta_{1}, \ldots, \theta_{K}\right)^{\prime}$ é o vetor dos parâmetros com dimensões $K \times 1$ e $\boldsymbol{\varepsilon}$ é um vetor $n \times 1$ dos erros experimentais. Dessa forma, (11) tem o seguinte aspecto, 


$$
\left[\begin{array}{c}
y_{11} \\
y_{12} \\
\vdots \\
y_{1 R} \\
y_{21} \\
y_{22} \\
\vdots \\
y_{2 R} \\
\vdots \\
\vdots \\
y_{K 1} \\
y_{K 2} \\
\vdots \\
y_{K R}
\end{array}\right]=\left[\begin{array}{cccc}
1 & 0 & \cdots & 0 \\
1 & 0 & \cdots & 0 \\
\vdots & \vdots & \ddots & \vdots \\
1 & 0 & \cdots & 0 \\
0 & 1 & \cdots & 0 \\
0 & 1 & \cdots & 0 \\
\vdots & \vdots & \ddots & \vdots \\
0 & 1 & \cdots & 0 \\
\vdots & \vdots & \ddots & \vdots \\
\vdots & \vdots & \ddots & \vdots \\
0 & 0 & \cdots & 1 \\
0 & 0 & \cdots & 1 \\
\vdots & \vdots & \ddots & \vdots \\
0 & 0 & \cdots & 1
\end{array}\right]\left[\begin{array}{c}
\theta_{1} \\
\theta_{2} \\
\vdots \\
\theta_{K}
\end{array}\right]+\left[\begin{array}{c}
e_{11} \\
e_{12} \\
\vdots \\
e_{1 R} \\
e_{21} \\
e_{22} \\
\vdots \\
e_{2 R} \\
\vdots \\
\vdots \\
e_{K 1} \\
e_{K 2} \\
\vdots \\
e_{K R}
\end{array}\right]
$$

Além disso, supõe-se que no modelo (11), $\varepsilon$ tem distribuição normal esfericamente multivariada $N_{n}\left(\mathbf{0}, \sigma^{2} I_{n}\right)$ e que a matriz $X$ tem posto $K$.

\subsubsection{A Eunção de Verossimilhança}

Levando-se em consideração o modelo (11) e a suposição de independència e normalidade dos erros experimentais, isto é,

$$
\varepsilon=y-X \theta \sim N_{n}\left(\mathbf{0}, \sigma^{2} I_{n}\right) \Longleftrightarrow y \sim N_{n}\left(X \boldsymbol{\theta}, \sigma^{2} I_{n}\right),
$$

então. a. distribuição conjunta de $\boldsymbol{y}$ dado $\left(\boldsymbol{\theta}, \sigma^{2}\right)$, será

$$
p\left(\boldsymbol{y} \mid \boldsymbol{\theta}, \sigma^{2}\right)=\left(2 \pi \sigma^{2}\right)^{-\frac{n}{2}} \exp \left[-\frac{1}{2 \sigma^{2}}(\boldsymbol{y}-X \boldsymbol{\theta})^{\prime}(\boldsymbol{y}-X \boldsymbol{\theta})\right] .
$$

Mas. 


$$
\begin{gathered}
(\boldsymbol{y}-X \boldsymbol{\theta})^{\prime}(\boldsymbol{y}-X \boldsymbol{\theta})=(\boldsymbol{y}-\hat{\boldsymbol{y}}+\hat{\boldsymbol{y}}-X \boldsymbol{\theta})^{\prime}(\boldsymbol{y}-\hat{\boldsymbol{y}}+\hat{\boldsymbol{y}}-X \boldsymbol{\theta})= \\
=\left[\nu S^{2}+(\boldsymbol{\theta}-\hat{\boldsymbol{\theta}})^{\prime} X^{\prime} X(\boldsymbol{\theta}-\hat{\boldsymbol{\theta}})\right],
\end{gathered}
$$

onde $\hat{\boldsymbol{\theta}}=\left(X^{\prime} X\right)^{-1} X^{\prime} \boldsymbol{y}$ é o estimador de máxima verossimilhança ou de mínimos quadrados de $\boldsymbol{\theta}$ (o vetor dos efeitos médios dos tratamentos ou paràmetros de locação), $\hat{\boldsymbol{y}}=X \hat{\boldsymbol{\theta}}$ são estimadores das observações individuais (valores preditos), $\nu=K(R-1)$ são os graus de liberdade associados ao resíduo e $S^{2}=m_{e}=$ $\frac{1}{\nu}(\boldsymbol{y}-\hat{\boldsymbol{y}})^{\prime}(\boldsymbol{y}-\hat{\boldsymbol{y}})$ é o estimador da variância residual cujo valor do quadrado médio residual é sua estimativa.

Dessa forma (13) pode ser escrita como

$$
\begin{aligned}
p\left(\boldsymbol{y} \mid \boldsymbol{\theta}, \sigma^{2}\right)= & \left(2 \pi \sigma^{2}\right)^{-\frac{n}{2}} \exp \left\{-\frac{1}{2 \sigma^{2}}\left[\nu S^{2}+(\boldsymbol{\theta}-\hat{\boldsymbol{\theta}})^{\prime} X^{\prime} X(\boldsymbol{\theta}-\hat{\boldsymbol{\theta}})\right]\right\} \\
& \sigma^{2}>0, \quad-\infty<\theta_{i}<+\infty, \quad i=1, \ldots, K .
\end{aligned}
$$

Agora, a função de verossimilhança fica,

$$
\ell\left(\boldsymbol{\theta}, \sigma^{2} \mid \boldsymbol{y}\right)=\left(2 \pi \sigma^{2}\right)^{-\frac{n}{2}} \exp \left\{-\frac{1}{2 \sigma^{2}}\left[\nu s^{2}+(\boldsymbol{\theta}-\hat{\boldsymbol{\theta}})^{\prime} X^{\prime} X(\boldsymbol{\theta}-\hat{\boldsymbol{\theta}})\right]\right\}
$$

Por Box \& Tiao (1973), as estatísticas $\hat{\boldsymbol{\theta}}$ e $S^{2}$ tèm as seguintes propriedades:

a) $\dot{\boldsymbol{\theta}}$ é um vetor $K \times 1$ de estatísticas conjuntamente suficientes para $\boldsymbol{\theta}$ se $\sigma^{2}$ for conhecido;

b) $\dot{\boldsymbol{\theta}}$ e $S^{2}$ são estatísticas conjuntamente suficientes para $\left(\boldsymbol{\theta}, \sigma^{2}\right)$;

c) $\grave{\boldsymbol{\theta}}$ tem distribuição normal multivariada $N_{K}\left(\boldsymbol{\theta}, \sigma^{2}\left(X^{\prime} X\right)^{-1}\right)$;

d) $\nu S^{2}$ é distribuída independentemente de $\hat{\boldsymbol{\theta}}$ como $\sigma^{2} \chi_{\nu}^{2}$.

Devido ao fato de que na experimentaçāo agronômica $\sigma^{2}$ é raramente conhecida, decidiu-se neste trabalho enfocar apenas o caso onde esta é desconhecida. 


\subsubsection{A Função de Distribuição a Priori de $\left(\boldsymbol{\theta}, \sigma^{2}\right)$}

Neste trabalho é considerado o caso onde o conhecimento do Pesquisador acerca dos paràmetros $\left(\boldsymbol{\theta}, \sigma^{2}\right)$ não é suficiente para avaliar o comportamento probabilístico destes paràmetros. Esta é uma situação bastante realística em se tratando de experimentos agronòmicos. Por esta razão decidiu-se admitir uma priori não-informativa para $\left(\boldsymbol{\theta}, \sigma^{2}\right)$, onde $(\boldsymbol{\theta}, \log \sigma)$ são aproximadamente independentes e localmente uniformes, isto é, conforme Box \& TiaO (1973),

$$
p\left(\boldsymbol{\theta}, \sigma^{2}\right) \doteq p(\theta) p\left(\sigma^{2}\right) \propto \frac{1}{\sigma^{2}}
$$

\subsubsection{A Função de Distribuição Conjunta a Posteriori de $\left(\boldsymbol{\theta}, \sigma^{2}\right)$}

Combinando a verossimilhança com a distribuição a priori, conforme a fórmula de Bayes em (4), tem-se

$$
\begin{aligned}
p\left(\boldsymbol{\theta}, \sigma^{2} \mid \boldsymbol{y}\right) & \propto p\left(\boldsymbol{\theta}, \sigma^{2}\right) p\left(\boldsymbol{y} \mid \boldsymbol{\theta}, \sigma^{2}\right) \\
& \propto(2 \pi)^{-\frac{n}{2}}\left(\sigma^{2}\right)^{-\left(\frac{n}{2}+1\right)} \exp \left\{-\frac{1}{2 \sigma^{2}}\left[\nu s^{2}+(\boldsymbol{\theta}-\hat{\boldsymbol{\theta}})^{\prime} X^{\prime} X(\boldsymbol{\theta}-\hat{\boldsymbol{\theta}})\right]\right\}
\end{aligned}
$$

ou ainda

$$
\begin{aligned}
p\left(\boldsymbol{\theta}, \sigma^{2} \mid \boldsymbol{y}\right)= & W(2 \pi)^{-\frac{n}{2}}\left(\sigma^{2}\right)^{-\left(\frac{n}{2}+1\right)} \exp \left\{-\frac{1}{2 \sigma^{2}}\left[\nu s^{2}+(\boldsymbol{\theta}-\hat{\boldsymbol{\theta}})^{\prime} X^{\prime} X(\boldsymbol{\theta}-\hat{\boldsymbol{\theta}})\right]\right\} . \\
& \sigma^{2}>0, \quad-\infty<\theta_{i}<+\infty, \quad i=1, \ldots, K
\end{aligned}
$$

onde $W$ é a constante normalizadora, obtida por

$$
\begin{aligned}
W^{-1}= & (2 \pi)^{-\frac{n}{2}} \int_{0}^{\infty}\left(\sigma^{2}\right)^{-\left(\frac{n}{2}+1\right)} \exp \left\{-\frac{\nu s^{2}}{2 \sigma^{2}}\right\} \times \\
& \int_{-\infty}^{+\infty} \exp \left\{-\frac{1}{2 \sigma^{2}}(\boldsymbol{\theta}-\hat{\boldsymbol{\theta}})^{\prime} X^{\prime} X(\boldsymbol{\theta}-\hat{\boldsymbol{\theta}})\right\} d \boldsymbol{\theta} d \sigma^{2} .
\end{aligned}
$$

Mas, sendo $\hat{\boldsymbol{\theta}} \sim N_{K}\left(\boldsymbol{\theta}, \sigma^{2}\left(X^{\prime} X\right)^{-1}\right)$, então

$$
\frac{\left|X^{\prime} X\right|^{\frac{1}{2}}}{\left(2 \pi \sigma^{2}\right)^{\frac{k^{\prime}}{2}}} \int_{-\infty}^{+\infty} \exp \left\{-\frac{1}{2 \sigma^{2}}(\boldsymbol{\theta}-\hat{\boldsymbol{\theta}})^{\prime} X^{\prime} X(\boldsymbol{\theta}-\hat{\boldsymbol{\theta}})\right\} d \boldsymbol{\theta}=1,
$$

então

$$
W^{-1}=\frac{(2 \pi)^{-\frac{n}{2}}(2 \pi)^{\frac{K}{2}}}{\mid X^{\prime} X^{\frac{1}{2}}} \int_{0}^{\infty}\left(\sigma^{2}\right)^{-\left(\frac{n}{2}+1\right)+\frac{K}{2}} \exp \left\{-\frac{\nu s^{2}}{2 \sigma^{2}}\right\} d \sigma^{2}
$$


Fazendo a substituição $U=\sigma^{2} / \nu S^{2}$ e integrando, encontra-se

$$
W=\frac{\left|X^{\prime} X\right|^{\frac{1}{2}}(2 \pi)^{\frac{\nu}{2}}\left(\nu s^{2}\right)^{\frac{\nu}{2}}}{\Gamma\left(\frac{\nu}{2}\right) 2^{\frac{\nu}{2}}}
$$

Finalmente, substituindo-se (23) em (19) obtém-se a distribuição conjunta a posteriori de $\left(\boldsymbol{\theta}, \sigma^{2}\right)$, a saber,

$$
\begin{aligned}
& p\left(\boldsymbol{\theta}, \sigma^{2} \mid \boldsymbol{y}\right)= \\
& =\frac{\left|X^{\prime} X\right|^{\frac{1}{2}}}{\left(2 \pi \sigma^{2}\right)^{\frac{K}{2}}} \frac{\left(\nu s^{2}\right)^{\frac{\nu}{2}}}{\Gamma\left(\frac{y}{z}\right) 2^{\frac{\nu}{2}}}\left(\sigma^{2}\right)^{-\left(\frac{\nu}{2}+1\right)} \exp \left\{-\frac{1}{2 \sigma^{2}}\left\{\nu s^{2}+(\boldsymbol{\theta}-\hat{\boldsymbol{\theta}})^{\prime} X^{\prime} X(\boldsymbol{\theta}-\hat{\boldsymbol{\theta}})\right]\right\}, \\
& \sigma^{2}>0, \quad-\infty<\theta_{i}<+\infty, \quad i=1, \ldots, K .
\end{aligned}
$$

\subsubsection{Distribuição Marginal a Posteriori de $\sigma^{2}$}

A distribuição marginal a posteriori de $\sigma^{2}$ é obtida integrando-se (24) em relação a $\boldsymbol{\theta}$, isto é,

$$
\begin{aligned}
p\left(\sigma^{2} \mid \boldsymbol{y}\right)= & \frac{\left(\nu s^{2}\right)^{\frac{\nu}{2}}}{\Gamma\left(\frac{\nu}{2}\right) 2^{\frac{\nu}{2}}}\left(\sigma^{2}\right)^{-\left(\frac{\nu}{2}+1\right)} \exp \left\{-\frac{\nu s^{2}}{2 \sigma^{2}}\right\} \times \\
& \frac{\left.\underbrace{\prime} X\right|^{\frac{1}{2}}}{\left(2 \pi \sigma^{2}\right)^{\frac{K}{2}}} \int_{-\infty}^{+\infty} \exp \left\{-\frac{1}{2 \sigma^{2}}(\boldsymbol{\theta}-\hat{\boldsymbol{\theta}})^{\prime} X^{\prime} X(\boldsymbol{\theta}-\hat{\boldsymbol{\theta}})\right\} d \boldsymbol{\theta}
\end{aligned}
$$

Portanto,

$$
p\left(\sigma^{2} \mid y\right)=\frac{\left(\nu s^{2}\right)^{-1}}{\Gamma\left(\frac{\nu}{2}\right) 2^{\frac{\nu}{2}}}\left(\frac{\sigma^{2}}{\nu s^{2}}\right)^{-\left(\frac{\nu}{2}+1\right)} \exp \left\{-\frac{\nu s^{2}}{2 \sigma^{2}}\right\} I_{(0, \infty)}\left(\sigma^{2}\right)
$$

e segue que

$$
\begin{gathered}
E\left(\sigma^{2} \mid \boldsymbol{y}\right)=\frac{\nu s^{2}}{\nu-1}, \quad \operatorname{Var}\left(\sigma^{2} \mid \boldsymbol{y}\right)=\frac{2\left(\nu s^{2}\right)^{2}}{(\nu-2)^{2}(\nu-4)} \\
M o\left(\sigma^{2} \mid \boldsymbol{y}\right)=\frac{\nu s^{2}}{\nu+2} \text { e } \int_{0}^{M d} p\left(\sigma^{2} \mid \boldsymbol{y}\right) d \sigma^{2}=0,5
\end{gathered}
$$

Fazendo a transformação $Z=\frac{\nu s^{2}}{\sigma^{2}}$, conclui-se que a distribuição $a$ posteriori de Z é

$$
p\left(Z=\frac{\nu s^{2}}{\sigma^{2}} \mid y\right)=\frac{1}{\Gamma\left(\frac{\nu}{2}\right) 2^{\frac{\nu}{2}}} z^{\frac{\nu}{2}-1} e^{-z / 2} I_{(0, \infty)}(z)
$$


a qual é a densidade de uma variável aleatória com distribuição de qui-quadrado com $\nu$ graus de liberdade, ou seja, $Z=\frac{\nu s^{2}}{\sigma^{2}}=\frac{S Q R e s .}{\sigma^{2}}$ é distribuída a posteriori como uma $\chi_{2}^{2}$. Intervalos de credibilidade a posteriori de conteúdo $(1-\alpha)$ de probabilidade para $\sigma^{2}$ poderão ser feitos através de $Z$ utilizando as tabelas usuais da distribuição de $\chi^{2}$. Assim, para $0<\eta_{1}<\eta_{2}<\infty$, tem-se

$$
\operatorname{Pr}\left\{\eta_{1}<\frac{\nu s^{2}}{\sigma^{2}} \mid y<\eta_{2}\right\}=1-\alpha
$$

e consequentemente

$$
\operatorname{Pr}\left\{\frac{\nu s^{2}}{\eta_{2}}<\sigma^{2} \mid y<\frac{\nu s^{2}}{\eta_{1}}\right\}=1-\alpha
$$

onde $\eta_{1}$ e $\eta_{2}$ são obtidos das tabelas usuais da distribuição da qui-quadrado com $\nu$ graus de liberdade.

\subsubsection{A Distribuição Marginal a Posteriori de $\theta$}

De modo análogo, a distribuição a posteriori de $\boldsymbol{\theta}$ é obtida integrando-se (24) em relação a $\sigma^{2}$, ou seja,

$$
p(\boldsymbol{\theta} \mid \boldsymbol{y})=\frac{\left|X^{\prime} X\right|^{\frac{1}{2}}\left(\nu s^{2}\right)^{\frac{\nu}{2}}}{(2 \pi)^{\frac{K}{2}} \Gamma\left(\frac{\nu}{2}\right) 2^{\frac{\nu}{2}}} \int_{0}^{\infty}\left(\sigma^{2}\right)^{-\left(\frac{n}{2}+1\right)} \exp \left\{-\frac{M(\boldsymbol{\theta})}{2 \sigma^{2}}\right\} d \sigma^{2}
$$

onde $M(\boldsymbol{\theta})=\left[\nu s^{2}+(\boldsymbol{\theta}-\hat{\boldsymbol{\theta}})^{\prime} X^{\prime} X(\boldsymbol{\theta}-\hat{\boldsymbol{\theta}})\right], n=\nu+K$. Fazendo a mudança de variável $\sigma^{2}=M(\boldsymbol{\theta}) U$ em (30) e fazendo algumas operações algébricas encontra-se a densidade a posteriori de $\boldsymbol{\theta}$, isto é,

$$
\begin{aligned}
p(\boldsymbol{\theta} \mid \boldsymbol{y})= & \frac{\Gamma\left(\frac{\nu+K}{2}\right) \mid X^{\prime} X^{\frac{1}{2}} s^{-K}}{\left[\Gamma\left(\frac{l}{2}\right)\right]^{K} \Gamma\left(\frac{\nu}{2}\right) \nu^{\frac{K}{2}}}\left[1+(\boldsymbol{\theta}-\hat{\boldsymbol{\theta}})^{\prime} X^{\prime} X\right. \\
\nu s^{2} & (\boldsymbol{\theta}-\hat{\boldsymbol{\theta}})]^{-\frac{\nu+K}{2}} \\
& -\infty<\theta_{i}<+\infty, \quad i=1, \ldots, K
\end{aligned}
$$

a qual é a densidade de uma t-Student multivariada $K$-dimensional com média $\hat{\boldsymbol{\theta}}$, matriz de dispersão $s^{2}\left(X^{\prime} X\right)^{-1}$ e $\nu$ graus de liberdade. Esta densidade é comumente denotada por $\boldsymbol{\theta} \mid \boldsymbol{y} \sim t_{K}\left(\hat{\boldsymbol{\theta}}, s^{2}\left(X^{\prime} X\right)^{-1}, \nu\right)$. 


\subsubsection{Região de Alta Densidade a Posteriori}

Para facilitar as discussões posteriores acerca do vetor de paràme$\operatorname{tros} \theta$, achou-se conveniente introduzir, neste momento, o conceito de Intervalos ou Região de Alta Densidade a posteriori (intervalos ou Regiões H.P.D.).

Do ponto de vista Bayesiano, todos os problemas inferenciais acerca de $\boldsymbol{\theta}$ poderão ser resolvidos através da densidade a posteriori $p(\boldsymbol{\theta} \mid \boldsymbol{y})$.

Uma maneira de pesquisar a informação contida em $p(\boldsymbol{\theta} \mid \boldsymbol{y})$ é observar que, apesar da densidade ser não nula sobre toda amplitude do espaço paramétrico, há uma parte substancial do espaço onde a densidade é negligenciável. Assim, é possível delimitar uma região de interesse (ou regiões), relativamente pequena, a qual contém a maior parte das probabilidades a posteriori. Obviamente existem inúmeras maneiras de se escolherem tais regiões. Em alguns casos, uma região pode ser escolhida pela natureza do problema. Quando este não for o caso, pode-se decidir que propriedades devem ter estas regiões. Tais como os intervalos ou regiōes H.P.D. definidos por Box \& TIAO (1973) como,

Definição: Seja $p(\boldsymbol{\theta} \mid \boldsymbol{y})$ uma função de densidade a posteriori. Uma região $\mathcal{R}$ no espaço paramétrico de $\theta$ é chamada região H.P.D. de conteúdo $(1-\alpha)$ se

(a) $\operatorname{Pr}\{\boldsymbol{\theta} \in \mathcal{R} \mid \boldsymbol{y}\}=1-\alpha$,

(b) Para $\boldsymbol{\theta}_{1} \in \mathcal{R}$ e $\boldsymbol{\theta}_{2} \notin \mathcal{R}, p\left(\boldsymbol{\theta}_{1} \mid \boldsymbol{y}\right) \geq p\left(\boldsymbol{\theta}_{2} \mid \boldsymbol{y}\right)$.

Com as propriedades,

1. Pela definição tem-se que para um dado conteúdo $(1-\alpha)$, a região H.P.D. tem o menor volume possível no espaço paramétrico de $\boldsymbol{\theta}$;

2. Suponha que $p(\boldsymbol{\theta} \mid \boldsymbol{y})$ seja não uniforme sobre toda região no espaço de $\boldsymbol{\theta}$, então a região H.P.D. de conteúdo $(1-\alpha)$ é única. Além disso, se $\boldsymbol{\theta}_{1}$ e $\boldsymbol{\theta}_{2}$ forem dois pontos tais que $p\left(\boldsymbol{\theta}_{1} \mid \boldsymbol{y}\right)=p\left(\boldsymbol{\theta}_{2} \mid \boldsymbol{y}\right)$, então estes dois pontos estarão simultaneamente incluídos (ou excluídos) da região H.P.D. de conteúdo $(1-\alpha)$. $A$ recíproca também é verdadeira. Isto é, se $p\left(\boldsymbol{\theta}_{1} \mid \boldsymbol{y}\right) \neq p\left(\boldsymbol{\theta}_{2} \mid \boldsymbol{y}\right)$, então existe 
uma região H.P.D. de conteúdo $(1-\alpha)$ a qual inclui um ponto mas não inclui o outro.

\subsubsection{Propriedades da Distribuição a Posteriori de $\theta$}

De acordo com Zellner (1971), Box \& TiaO (1973), dentre outros, $p(\boldsymbol{\theta} \mid \boldsymbol{y})$ tem as seguintes propriedades:

P.1) A função $p(\boldsymbol{\theta} \mid \boldsymbol{y})$ é monotonicamente decrescente da formá quadrática $Q(\boldsymbol{\theta})=$ $(\boldsymbol{\theta}-\hat{\boldsymbol{\theta}})^{\prime} X^{\prime} X(\boldsymbol{\theta}-\hat{\boldsymbol{\theta}})$. Assim, $Q(\boldsymbol{\theta})=c, c>0$, define os contornos da distribuição no espaço $K$-dimensional de $\boldsymbol{\theta}$. Para $K=2, Q(\boldsymbol{\theta})=c$ é a equação de uma elipse; para $K=3$ ela é uma elipsóide e para $K>3$ ela é a equação de uma hiper-elipsóide;

P.2) A quantidade $Q(\boldsymbol{\theta}) / K s^{2}$ é distribuída a posteriori como uma $F_{[K, \nu]}$. Os contornos elipsoidais de $p(\boldsymbol{\theta} \mid \boldsymbol{y})$ serão definidos por

$$
\frac{Q(\boldsymbol{\theta})}{K s^{2}}=F_{[K, \nu, \alpha]}
$$

onde $F_{[K, \nu, \alpha]}$ é o ponto superior de uma distribuição $F \operatorname{com}(K, \nu)$ graus de liberdade que delimita uma região elipsoidal no espaço $K$-dimensional de $\boldsymbol{\theta}$, a qual contém $(1-\alpha)$ de probabilidade $a$ posteriori. Tal região é definida pela inequação

$$
\frac{Q(\boldsymbol{\theta})}{K s^{2}}<F_{[K, \nu, \alpha]}
$$

P.3) Seja a partição

$$
\begin{aligned}
& \boldsymbol{\theta}=\left[\begin{array}{c}
\boldsymbol{\theta}_{1} \\
\cdots \\
\boldsymbol{\theta}_{2}
\end{array}\right] \begin{array}{c}
r \\
K-r
\end{array} ; \quad \hat{\boldsymbol{\theta}}=\left[\begin{array}{c}
\hat{\boldsymbol{\theta}}_{1} \\
\ldots \\
\hat{\boldsymbol{\theta}}_{2}
\end{array}\right] \begin{array}{c}
r \\
K-r
\end{array} \\
& X^{\prime} X=\left[\begin{array}{cc}
X_{1}^{\prime} X_{1} & X_{1}^{\prime} X_{2} \\
X_{2}^{\prime} X_{1} & X_{2}^{\prime} X_{2}
\end{array}\right] \begin{array}{c}
r \\
K-r
\end{array} \quad e \quad\left(X^{\prime} X\right)^{-1}=\left[\begin{array}{cc}
C_{11} & C_{12} \\
C_{21} & C_{22}
\end{array}\right] \begin{array}{c}
r \\
K-r
\end{array} \text {. }
\end{aligned}
$$


De conformidade com as propriedades da distribuição t-Student multivariada, ZELLNER (1971), se $\boldsymbol{\theta}$ tem distribuição t-Student multivariada $K$ dimensional, com vetor de médias $\hat{\boldsymbol{\theta}}$, matriz de dispersão $s^{2}\left(X^{\prime} X\right)^{-1}$ e $\nu$ graus de liberdade, então um subconjunto $\boldsymbol{r}$-dimensional $\boldsymbol{\theta}_{1}$, tem distribuição t-Student multivariada $r$-dimensional com vetor de médias $\hat{\theta_{1}}$, matriz de dispersão $s^{2} C_{11}$ e $\nu$ graus de liberdade, denotada por $\boldsymbol{\theta}_{1} \mid \boldsymbol{y} \sim t_{r}\left[\hat{\boldsymbol{\theta}}_{1}, s^{2} C_{11}, \nu\right]$, cuja função de densidade $a$ posteriorié

$$
\begin{aligned}
p\left(\boldsymbol{\theta}_{1} \mid y\right)= & \frac{\Gamma\left(\frac{\nu+r}{2}\right)\left|C_{11}^{-1}\right|^{\frac{1}{2}} s^{-r}}{\left[\Gamma\left(\frac{1}{2}\right)\right]^{r} \Gamma\left(\frac{\nu}{2}\right) \nu^{\frac{r}{2}}}\left[1+\left(\boldsymbol{\theta}_{1}-\hat{\theta_{1}}\right)^{\prime} \frac{C_{11}^{-1}}{\nu s^{2}}(\boldsymbol{\theta}-\hat{\boldsymbol{\theta}})\right]^{-\left(\frac{\nu+r}{2}\right)}, \\
& -\infty<\theta_{i}<+\infty, \quad i=1, \ldots, r
\end{aligned}
$$

Em particular, a distribuição marginal a posteriori de $\theta_{i}, \forall i, \mathrm{i}=1, \ldots, \mathrm{K}$ é dada por

$$
\begin{aligned}
p\left(\theta_{i} \mid y\right)= & \frac{\Gamma\left(\frac{\nu+1}{2}\right)\left|c_{i i}^{-1}\right|^{\frac{1}{2}} s^{-1}}{\Gamma\left(\frac{1}{2}\right) \Gamma\left(\frac{\nu}{2}\right) \nu^{\frac{1}{2}}}\left[1+\frac{\left(\theta_{i}-\hat{\theta_{i}}\right)^{2}}{\nu s^{2} c_{i i}}\right]^{-\left(\frac{\nu+1}{2}\right)} \\
& -\infty<\theta_{i}<+\infty, \forall \theta_{i}, \quad i=1, \ldots, K
\end{aligned}
$$

isto é, $\theta_{i} \mid \boldsymbol{y} \sim t_{1}\left[\hat{\theta}_{i}, s^{2} c_{i i}, \nu\right], \quad \forall i, \quad i=1, \ldots, K$.

Além disso, a variável $t=\frac{\theta_{i}-\hat{\theta}_{i}}{\sqrt{s^{2} c_{i i}}}$ é distribuída a posteriori como uma t-Student padrão com $\nu$ graus de liberdade e intervalos H.P.D. para $\theta_{i}$ podem ser construídos empregando as tabelas usuais da distribuição t-Student padrão, a partir da expressão

$$
\operatorname{Pr}\left[\hat{\theta}_{i}-t_{\frac{\alpha}{2}} \sqrt{s^{2} c_{i i}} \leq \theta_{i} \leq \hat{\theta}_{i}+t_{\frac{\alpha}{2}} \sqrt{s^{2} c_{i i}}\right]=1-\alpha,
$$

onde, para o modelo aqui considerado, $\hat{\theta}_{i}=\bar{y}_{i}$ é a média das observações que receberam o tratamento $i, c_{i i}=1 / R$ é o $i$-ésimo elemento da diagonal da matriz $\left(X^{\prime} X^{r}\right)^{-1}$ e $s^{2}=$ QM.Resíduo.

P.4) A distribuição condicional a posteriori de um subconjunto $(K-r)$ dos parâmetros $\boldsymbol{\theta}_{2}$ dado $\boldsymbol{\theta}_{1}$ é também t-Student multivariada $(K-r)$-dimensional. Especificamente, $\boldsymbol{\theta}_{2}$ dado $\boldsymbol{\theta}_{1}$ é distribuida como

$$
t_{(K-r)}\left[\hat{\theta}_{2.1}, s_{2.1}^{2}\left(X_{2}^{\prime} X_{2}\right)^{-1}, \nu+r\right]
$$


onde

$$
\begin{array}{r}
\hat{\boldsymbol{\theta}}_{2.1}=\hat{\boldsymbol{\theta}}_{2}-\left(X_{2}^{\prime} X_{2}\right)^{-1}\left(X_{2}^{\prime} X_{1}\right)\left(\boldsymbol{\theta}_{1}-\hat{\boldsymbol{\theta}}_{1}\right) e \\
s_{2.1}^{2}=(\nu+r)^{-1}\left[\nu s^{2}+\left(\boldsymbol{\theta}_{1}-\hat{\boldsymbol{\theta}}_{1}\right)^{\prime} C_{11}^{-1}\left(\boldsymbol{\theta}_{1}-\hat{\boldsymbol{\theta}}_{1}\right)\right] .
\end{array}
$$

P.5) Suponha que $\phi=\left(\phi_{1}, \ldots, \phi_{m}\right), m<K$, seja um conjunto de combinações lineares tais que

$$
\phi=D \theta
$$

onde $D$ é uma matriz $m \times k$ de posto $m$. Então $\phi$ é distribuído a posteriori como

$$
t_{m}\left[D \hat{\boldsymbol{\theta}}, s^{2} D\left(X^{\prime} X\right)^{-1} D^{\prime}, \nu\right] .
$$

Esta propriedade é de grande importância para os objetivos deste trabalho, pois, ela permite que os parâmetros sejam comparados entre si, o que na grande maioria das aplicações práticas é mais interessante do que simplesmente determinar seus valores absolutos.

Suponham-se $K$ parâmetros $\boldsymbol{\theta}=\left(\theta_{1}, \ldots, \theta_{K}\right)^{\prime}$. Em geral, na prática, definem-se $(K-1)$ comparações não redundantes através de $(K-1)$ funções independentes do tipo

$$
\phi_{j}=f_{j}(\boldsymbol{\theta}), \quad j=1,2, \ldots,(K-1),
$$

as quais serão todas iguais a zero se, e somente se, $\theta_{1}=\theta_{2}=\ldots=\theta_{K}$. Obviamente existe uma infinidade de funções com estas características, dentre elas, os contrastes ortogonais são de particular interesse na estatística experimental. Assim, afirmações sobre os valores comparativos dos $\theta_{i}$ 's poderão ser feitas através de $(K-1)$ contrastes ortogonais $\phi_{1}, \ldots, \phi_{(K-1)}$. Em particular, a probabilidade de que todos os $\theta_{i}$ 's são iguais, é igual à probabilidade de que todos os $\phi_{i}$ 's são nulos, isto é, à probabilidade de que $\theta_{1}=\ldots=\theta_{K}$ é igual a probabilidade de $\phi_{1}=\ldots=\phi_{(K-1)}=0$. 
De acordo com (41), segue que a posteriori

$$
\boldsymbol{\phi}=\left[\begin{array}{c}
\phi_{1} \\
\phi_{2} \\
\vdots \\
\phi_{(K-1)}
\end{array}\right] \sim t_{(K-1)}\left[\hat{\phi}=D \hat{\boldsymbol{\theta}}, s^{2} D\left(X^{\prime} X\right)^{-1} D^{\prime}, \nu\right]
$$

cuja função de densidade a posteriori é

$$
\begin{aligned}
p(\boldsymbol{\phi} \mid \boldsymbol{y})= & \frac{\Gamma\left(\frac{\nu+K^{\prime}-1}{2}\right)\left|D\left(X^{\prime} X\right)^{-1} D^{\prime}\right|^{-\frac{1}{2}} s^{-(K-1)}}{\left[\Gamma\left(\frac{1}{2}\right)\right]^{K-1} \Gamma\left(\frac{\nu}{2}\right) 2^{\frac{K}{2}-1}} \times \\
& \left\{1+\frac{(\boldsymbol{\phi}-\hat{\phi})^{\prime}\left[D\left(X^{\prime} X\right)^{-1} D^{\prime}\right]^{-1}(\phi-\hat{\phi})}{\nu s^{2}}\right\}^{-\left(\frac{\nu+\frac{K}{2}-1}{2}\right)},
\end{aligned}
$$

onde $-\infty<\phi_{j}<+\infty, D=d_{j i}, j=1,2, \ldots,(K-1), i=1,2, \ldots, K$, é uma matriz com dimensões $(K-1) \times K$ de posto $(K-1)$, correspondente a $(K-1)$ contrastes não redundantes sobre os $K$ parâmetros (tratamentos).

Em particular, a distribuição marginal a posteriori de $\phi_{j}=\sum_{i=1}^{K} d_{j i} \theta_{i}$, $\forall j, \operatorname{com} \sum_{i=1}^{K} d_{j i}=0$ é t-Student univariada com média $\hat{\phi}_{j}$, variância $s^{2} h_{j j}$ e $\nu$ graus de liberdade. Isto é, $\phi_{j} \mid \boldsymbol{y} \sim t_{1}\left[\hat{\phi}_{j}, s^{2} h_{j j}, \nu\right]$, onde $h_{j j}$ é o $j$-ésimo elemento da diagonal da matriz $H=\left[D\left(X^{\prime} X\right)^{-1} D^{\prime}\right]$, ou seja, $h_{j j}=\frac{1}{R} \sum_{i=1}^{K} d_{j \mathrm{j}}^{2}$. A densidade a posteriori de $\phi_{j}$ é

$$
\begin{aligned}
p\left(\phi_{j} \mid \boldsymbol{y}\right)= & \frac{\Gamma\left(\frac{\nu+1}{2}\right)\left|h_{j j}\right|^{-\frac{1}{2}} s^{-1}}{\Gamma\left(\frac{1}{2}\right) \Gamma\left(\frac{\nu}{2}\right)(\nu)^{\frac{1}{2}}}\left[1+\frac{\left(\phi_{j}-\hat{\phi}_{j}\right)^{2}}{\nu s^{2} h_{j j}}\right]^{-\left(\frac{\nu+1}{2}\right)} \\
& -\infty<\phi_{j}<+\infty, \quad \forall j
\end{aligned}
$$

onde, neste trabalho, $\hat{\phi}_{j}=\sum_{i=1}^{K} d_{j i} \hat{\theta}_{i}=\sum_{i=1}^{K} d_{j i} \bar{y}_{i}$

Tal como em P.3), a variável aleatória

$$
t=\frac{\phi_{j}-\hat{\phi}_{j}}{s \sqrt{h_{j j}}}, \quad \forall j
$$

segue uma distribuição t-Student univariada padrão com $\nu$ graus de liberdade. A partir deste resultado, intervalos H.P.D. de conteúdo $(1-\alpha)$ podem ser construídos, 
empregando as tabelas usuais da distribuiçào t-Student padronizada, através da expressão

$$
\operatorname{Pr}\left[\hat{\phi}_{j}-t_{\frac{\alpha}{2}} s \sqrt{h_{j j}} \leq \phi_{j} \leq \hat{\phi}_{j}+t_{\frac{\alpha}{2}} s \sqrt{h_{j j}}\right]=1-\alpha
$$

\subsubsection{Inferências Conjunta e Marginal}

Conforme foi dito anteriormente, toda informação acerca dos paràmetros de interesse $\boldsymbol{\theta}$, estará contida na distribuição a posteriori $p(\boldsymbol{\theta} \mid \boldsymbol{y})$, e consequentemente, todos os problemas inferenciais acerca de $\boldsymbol{\theta}$ poderão ser resolvidos através de $p(\boldsymbol{\theta} \mid \boldsymbol{y})$. Neste trabalho, as inferèncias sobre os efeitos médios dos tratamentos $\boldsymbol{\theta}$ ou sobre combinações lineares deles $\boldsymbol{\phi}=\boldsymbol{D} \boldsymbol{\theta}$, serão feitas verificando se um determinado ponto $\theta_{0}$ (ou $\phi_{0}$ ), está ou nào incluído na região H.P.D. de conteúdo $(1-\alpha)$.

A distribuição marginal a posteriori de $\boldsymbol{\theta}$ em (31) é t-Student multivariada $K$-dimensional. Além disso, conforme P.2), a quantidade $\frac{Q(\theta)}{K^{2} s^{2}}$ é distribuída a posteriori como uma $F_{\{K, \nu]}$. Suponha agora que o interesse é verificar se o ponto $\theta_{0}=\left(\theta_{10}, \theta_{20}, \ldots, \theta_{K 0}\right)^{\prime}$, está ou não incluído na região H.P.D. de conteúdo $(1-\alpha)$. Para verificar isto, BOX \& TIAO (1973) sugerem que seja calculada a probabilidade do evento $p(\boldsymbol{\theta} \mid \boldsymbol{y})>p\left(\boldsymbol{\theta}_{0} \mid \boldsymbol{y}\right)$. Segue, portanto, que o ponto $\boldsymbol{\theta}_{0}$ estará incluído na regiào H.P.D. de conteúdo $(1-\alpha)$ se, e somente se, $\operatorname{Pr}\left[p(\boldsymbol{\theta} \mid \boldsymbol{y})>p\left(\boldsymbol{\theta}_{0} \mid \boldsymbol{y}\right)\right] \leq$ $(1-\alpha)$. Mas, sendo $p(\boldsymbol{\theta} \mid \boldsymbol{y})$ uma função monotonicamente decrescente da quantidade $\frac{Q(\boldsymbol{\theta})}{K^{2} s^{2}}=\frac{\left(\boldsymbol{\theta}-\hat{\theta}^{\prime}\right)^{\prime} X(\boldsymbol{\theta}-\hat{\boldsymbol{\theta}})}{K s^{2}}$, o ponto $\boldsymbol{\theta}_{0}$ estará incluído na região H.P.D. de conteúdo $(1-\alpha)$ se, e somente se,

$$
\left(\boldsymbol{\theta}_{0}-\hat{\boldsymbol{\theta}}\right)^{\prime} X^{\prime} X\left(\boldsymbol{\theta}_{0}-\hat{\boldsymbol{\theta}}\right)<K s^{2} F_{[K, \nu, \alpha]}
$$

Equivalentemente, a quantidade

$$
\operatorname{Pr}\left\{F_{[K, \nu]}<\frac{\left(\boldsymbol{\theta}_{0}-\dot{\boldsymbol{\theta}}\right)^{\prime} X^{\prime} X\left(\boldsymbol{\theta}_{0}-\hat{\boldsymbol{\theta}}\right)}{K s^{2}}\right\},
$$

onde $F_{[K, \nu]}$ é uma variável aleatória distribuída como uma $F \operatorname{com}(K, \nu)$ graus de liberdade, fornece o conteúdo da região H.P.D. a qual inclui o ponto $\boldsymbol{\theta}_{0}$. 
Também, a quantidade $\frac{Q(\phi)}{(K-1) s^{2}}=\frac{(\phi-\dot{\phi})^{\prime} D\left[\left(X^{\prime} X\right)^{-1} D^{\prime}\right]^{-1}(\phi-\dot{\phi})}{(K-1) s^{2}}$ é distribuída a posteriori como uma $F_{[(K-1), \nu]}$. Sendo assim, $p(\phi \mid y)$ em (44) é uma função monotonicamente decrescente da quantidade $\frac{Q(\phi)}{(K-1) s^{2}}$. Um ponto particular $\phi_{0}$ estará incluído na região H.P.D. de conteúdo $(1-\alpha)$ se, e somente se,

$$
\left(\phi_{0}-\hat{\phi}\right)^{\prime}\left[D\left(X^{\prime} X\right)^{-1} D^{\prime}\right]^{-1}\left(\phi_{0}-\hat{\phi}\right)<(K-1) s^{2} F_{[(K-1), \nu, \alpha]} \text {. }
$$

Análogo a (49), a quantidade

$$
\operatorname{Pr}\left\{F_{\{(K-1), \nu, \alpha\}}<\frac{\left(\phi_{0}-\hat{\phi}\right)^{\prime}\left[D\left(X^{\prime} X\right)^{-1} D^{\prime}\right]^{-1}\left(\phi_{0}-\hat{\phi}\right)}{(K-1) s^{2}}\right\}
$$

onde $F_{\{(K-1), \nu]}$ é uma variável aleatória distribuída como uma $F$ com $[(K-1), \nu]$ graus de liberdade, fornece o conteúdo da região H.P.D. a qual inclui o ponto $\phi_{0}$.

Os resultados (48) e (49) fornecem uma justificativa Bayesiana da análise da variància, bem como, (50) e (51). Também, (48) e (50) são apropriados para decidir se um determinado ponto $\boldsymbol{\theta}_{0}$ ou $\phi_{0}$ está fora ou dentro da região H.P.D. de conteúdo $(1-\alpha)$. Além disso, os complementos das probabilidades em (49) e (51), fornecem o nível de significància associado às hipóteses $H_{0}: \boldsymbol{\theta}=\boldsymbol{\theta}_{0}$ e $H_{0}: \boldsymbol{\phi}=\boldsymbol{\phi}_{0}$, respectivamente.

Caso haja interesse em calcular probabilidades acerca dos paràmetros $\theta_{i}, i=1,2, \ldots, K$ individualmente, ou de um subconjunto dos $K$ paràmetros, isso pode ser feito usando as distribuições marginais a posteriori. É importante não cometer o engano de dizer que as probabilidades conjuntas podem ser obtidas a partir das distribuiçōes marginais, pois os intervalos marginais de alta densidade a posteriori de conteúdo $(1-\alpha)$ podem incluir um determinado ponto e este estar excluído da região H.P.D. de conteúdo $(1-\alpha)$ quando considerada a distribuição conjunta a posteriori. Quando se leva em conta as densidades marginais a posteriori, o interesse será responder, por exemplo, a questão: qual a probabilidade de $\theta_{1}$ assumir um determinado valor independentemente do valor assumido pelos demais paràmetros? E, quando se considera a distribuição conjunta, o interesse estará no cálculo da probabilidade de que os paràmetros assumam determinados valores conjuntamente. 
Com relação à justificativa Bayesiana da análise de variância, pode ser tomado qualquer conjunto $(K-1)$ contrastes não redundantes dos parâmetros $\theta$ para verificar a hipótese $H_{0}: \phi=0$ vs $H_{1}: \phi \neq 0$ a um nível de significância $\alpha$, a qual é equivalente a verificar a hipótese $H_{0}: \theta_{1}=\cdots=\theta_{K}$ contra a hipótese alternativa $H_{1}$ : a igualdade não prevalece para algum $\theta_{i}$. Neste sentido, é conveniente considerar o particular conjunto de contrastes $\phi=D \boldsymbol{\theta}$ dado por

$$
\left[\begin{array}{c}
\phi_{1} \\
\phi_{2} \\
\vdots \\
\phi_{(K-1)}
\end{array}\right]=\frac{1}{K}\left[\begin{array}{ccccc}
K-1 & -1 & \cdots & -1 & -1 \\
-1 & K-1 & \ldots & -1 & -1 \\
\vdots & \vdots & \ddots & \vdots & \vdots \\
-1 & -1 & \cdots & K-1 & -1
\end{array}\right]\left[\begin{array}{c}
\theta_{1} \\
\theta_{2} \\
\vdots \\
\theta_{K}
\end{array}\right]=\left[\begin{array}{c}
\theta_{1}-\bar{\theta} \\
\theta_{2}-\bar{\theta} \\
\vdots \\
\theta_{(K-1)}-\bar{\theta}
\end{array}\right]
$$

Usando o conjunto de contrastes não redundantes em (52), a densidade a posteriori em (44) pode ser escrita como

$$
p(\phi \mid y) \propto\left\{1+\frac{Q(\phi)}{\nu s^{2}}\right\}^{-\frac{\nu+K-1}{2}}, \quad-\infty<\phi_{j}<+\infty, \quad j=1, \ldots,(K-1),
$$

onde $Q(\phi)=R \sum_{i=1}^{K}\left[\phi_{i}-\left(\bar{y}_{i .}-\bar{y}_{. .}\right)\right]^{2} ; \bar{y}_{i .}=\frac{1}{R} \sum_{r=1}^{R} y_{i r} ; \bar{y}_{. .}=\frac{1}{K R} \sum_{i=1}^{K} \sum_{r=1}^{R} y_{i r} ;$ $\phi_{K}=\theta_{K}-\bar{\theta} ; \sum_{i=1}^{K} \phi_{i}=0$.

Assim, o ponto particular $\phi_{0}=0$ estará incluído na região H.P.D. de conteúdo $(1-\alpha)$ se, e somente se,

$$
\frac{Q\left(\phi_{0}\right)}{(K-1) s^{2}}=\frac{Q(0)}{(K-1) s^{2}}=\frac{R \sum_{i=1}^{K}\left(\bar{y}_{i .}-\bar{y} .\right)^{2}}{(K-1) s^{2}}<F_{[(K-1), \nu, \alpha]},
$$

o qual é um resultado paralelo ao teste de significância, cujos cálculos sào equivalentes aos da análise da variância, podem ser vistos na tabela 1.

Para testar hipóteses do tipo $H_{0}: \phi_{j}=0$ vs $H_{1}: \phi_{j} \neq 0, \forall j$, ao nível de significància $\alpha$, basta construir o intervalo H.P.D. de conteúdo $(1-\alpha)$ através da expressão (47) e verificar se o mesmo contém o ponto zero. Caso afirmativo, aceita-se $H_{0}$, caso contrário rejeita-se $H_{0}$ em favor de $H_{1}$.

\subsubsection{Comparação das Dispersões de K Populações Normais}

No modelo definido em (9), supõe-se que os erros experimentais são 
Tabela 1: Esquema da análise de variância para determinar se o ponto $\phi_{0}=0$ $\left(\theta_{1}=\theta_{2}=\cdots=\theta_{K}\right)^{\prime}$ está incluído na região H.P.D. que contém $100(1-\alpha) \%$ de probabilidade.

\begin{tabular}{|l|c|c|c|c|}
\hline \hline F. Variação & GL & SQ & QM & Razão \\
\hline \hline Igualdade das médias & $K-1$ & $Q(0)=R \sum_{i=1}^{K}\left(\bar{y}_{i .}-\bar{y}_{. .}\right)^{2}$ & $\frac{Q(0)}{K-1}$ & $\frac{Q(0)}{(K-1) S^{2}}$ \\
Resíduo & $\nu=K(R-1)$ & $\nu s^{2}=\sum_{i=1}^{K} \sum_{j=1}^{R}\left(y_{i j}-\bar{y}_{i .}\right)^{2}$ & $s^{2}$ & - \\
\hline \hline
\end{tabular}

distribuídos independentemente como uma normal multivariada $N_{n}\left(\mathbf{0}, I_{n} \sigma^{2}\right)$, isto é, supõe-se que a variância dentro de cada tratamento é a mesma $\left(\sigma_{1}^{2}=\sigma_{2}^{2}=\ldots=\sigma_{K}^{2}\right)$. Para verificar a hipótese de homogeneidade das variâncias, decidiu-se, neste trabalho, apresentar dois testes Bayesianos, a saber: O Teste de Bartlett Modificado, adaptado para o enfoque Bayesiano por Box \& TiaO(1973) e o Teste da Raiz Cúbica proposto por SAMIUdDIN (1976). Ambos são apropriados para verificar a hipótese de homogeneidade das variâncias de $K$ populações normais independentes $N\left(\theta_{i}, \sigma_{i}^{2}\right),(i=1,2, \ldots, K)$ e admitem que as distribuições a priori para $\theta_{i}$ e $\log \sigma_{i}$ são aproximadamente independentes e localmente uniformes. A idéia da apresentação do Teste da Raiz Cúbica é que ele é menos sensível à ausência de normalidade nos dados do que o Teste de Bartlett Modificado e, portanto, apresenta melhor desempenho nestes casos.

Os testes são baseados na escolha de $(K-1)$ contrastes lineares independentes em $\log \sigma_{i}$, isto é,

$$
\varphi_{i}=\log \sigma_{i}^{2}-\log \sigma_{K}^{2}=2\left(\log \sigma_{i}-\log \sigma_{K}\right), \quad i=1,2, \ldots,(K-1)
$$

Com base na distribuição a posteriori de $\varphi=\left(\varphi_{1}, \ldots, \varphi_{(K-1)}\right)$, os autores concluem que verificar a hipótese $H_{0}: \sigma_{1}^{2}=\ldots=\sigma_{K}^{2}$ a um nível de significància $\alpha$ é equivalente a verificar se o ponto $\varphi_{0}=0$ está dentro ou fora da região H.P.D. de conteúdo $(1-\alpha)$. E, portanto, o ponto $\varphi_{0}=\mathbf{0}$ estará incluído na região 
H.P.D. de conteúdo $(1-\alpha)$ se, e somente se,

$$
\begin{aligned}
& \frac{-2 \log W_{0}}{1+A}<\chi_{[(K-1), \alpha]}^{2}, \quad \text { (Teste de Bartlett Modificado): } \\
& \sum_{i=1}^{K} \frac{\left(m_{i}-m\right)^{2}}{a_{i}^{2}}<\chi_{\{(K-1), \alpha\}}^{2}, \quad \text { (Teste da Raiz Cúbica), }
\end{aligned}
$$

onde.

$$
\begin{array}{ll}
-2 \log W_{0}=-\sum_{i=1}^{K} \nu_{i}\left(\log s_{i}^{2}-\log \bar{s}^{2}\right), & \nu_{i}=R-1, \\
s_{i}^{2}=\frac{1}{\nu_{i}} \sum_{r=1}^{R}\left(y_{i r}-\bar{y}_{i .}\right)^{2}, & \bar{y}_{i}=\frac{1}{R} \sum_{r=1}^{R} y_{i r}, \\
\bar{s}^{2}=\frac{1}{\nu} \sum_{i=1}^{K} \nu_{i} s_{i}^{2}, & \nu=\nu_{1}+\nu_{2}+\cdots+\nu_{K}, \\
A=\frac{1}{3(K-1)}\left(\sum_{i=1}^{K} \nu_{i}^{-1}-\nu^{-1}\right), & m_{i}=\left(\nu_{i} / s_{i}^{2}\right)^{\frac{1}{3}}\left(1-2 / 9 \nu_{i}\right), \\
a_{i}^{2}=\frac{2}{9}\left(s_{i}^{2}\right)^{\frac{2}{3}}\left(\nu_{i}\right)^{\frac{1}{3}} \in & m=\frac{\sum_{i=1}^{K}\left(m_{i} / a_{i}^{2}\right)}{\sum_{i=1}^{K}\left(1 / a_{i}^{2}\right)} .
\end{array}
$$

Estes dois testes serão usados na verificação das hipóteses de homogeneidade das variâncias dentro e entre os ensaios.

\subsection{A Análise Conjunta de $J$ Experimentos}

Do ponto de vista Bayesiano, é possível se fazer a análise conjunta de um grupo de $J$ experimentos levando-se em consideração a natureza sequencial do Teorema de Bayes, ou seja, admitir, por exemplo, distribuição a priori não informativa para os paràmetro $\left(\boldsymbol{\theta}, \sigma^{2}\right)$ no primeiro ensaio, determinar a densidade

a posteriori e esta servir de priori para o segundo ensaio e assim por diante até o J-ésimo experimento. Porém esta análise contemplaria apenas as informações provenientes dos efeitos dos tratamentos e do erro experimental. A análise proposta neste trabalho, leva em consideração, além dos efeitos do fator tratamento e do erro experimental, os efeitos do fator experimento e da interação tratamento $\times$ experimento, os quais são de grande importância na experimentação. Considera tamēm, a homocedasticidade das variâncias dentro e entre os experimentos envolvidos na análise. Quando a suposição de homogeneidade das variâncias entre os experimentos não ocorrer, recomenda-se que os experimentos sejam agrupados em subgrupos cujos quadrados médios residuais seja homogèneos. 
De acordo com os objetivos deste trabalho, o modelo estrutural associado ao conjunto dos $J$ ensaios será um modelo misto com dois fatores cruzados.

\subsubsection{O Modelo Estatístico}

O modelo estatístico associado aos dados de um grupo de $J$ experimentos completamente aleatorizados, é dado por

$$
y_{i j r}=\mu+\alpha_{i}+\beta_{j}+\gamma_{i j}+e_{i j r}, \quad c o m\left\{\begin{array}{l}
i=1, \ldots, K, \\
j=1, \ldots, J \\
r=1, \ldots, R,
\end{array}\right.
$$

ou de acordo com (10)

$$
y_{i j r}=\theta_{i}+\beta_{j}+\gamma_{i j}+e_{i j r}, \quad c o m\left\{\begin{array}{l}
i=1, \ldots, K, \\
j=1, \ldots, J \\
r=1, \ldots, R,
\end{array}\right.
$$

onde $y_{i j r}$ é a $r$-ésima observação proveniente da unidade experimental que recebeu o tratamento $i$ no $j$-ésimo experimento; $\theta_{i}$ é o efeito médio do tratamento $i$, considerado fixo; $\beta_{j}$ é o efeito do $j$-ésimo experimento (ambiente, ano, estação, etc), considerado de efeito aleatório; $\gamma_{i j}$ é o efeito da interação do $i$-ésimo tratamento com o j-ésimo ensaio, também considerado de efeito aleatório e $e_{i j r}$ é o erro experimental, aleatório, associado à observação $y_{i j r}$.

Além disso, supõe-se que $\beta_{j}, \gamma_{i j}$ e $e_{i j r}$ são variáveis aleatórias independentes distribuídas como

$$
\begin{aligned}
& \beta_{j} \sim N\left(0, \sigma_{b}^{2}\right), \quad \gamma_{i j} \sim N\left(0, \sigma_{g}^{2}\right), \quad \text { e } e_{i j r} \sim N\left(0, \sigma_{e}^{2}\right) . \\
& \text { O modelo (60) pode ser representado na forma matricial por } \\
& \boldsymbol{y}=M \boldsymbol{\lambda}+\varepsilon, \quad \text { ou } \boldsymbol{y}=X \boldsymbol{\theta}+Z \boldsymbol{\beta}+G \boldsymbol{\gamma}+\boldsymbol{\varepsilon}
\end{aligned}
$$

onde $y$ é um vetor de realizações de variáveis aleatórias com dimensões $(K J R \times$ 1), $M$ é uma matriz de zeros e uns (ou matriz de planejamento) com dimensões 
$(K J R \times(K+J+K J)), \boldsymbol{\lambda}=\left(\theta_{1}, \cdots, \theta_{K}, \beta_{1}, \cdots, \beta_{J}, \gamma_{11}, \cdots, \gamma_{K J}\right)^{\prime}$ 'é um vetor de $(K+J+K J)$ parâmetros associados aos efeitos de $K$ tratamentos, $J$ experimentos e $K J$ interações, $\varepsilon$ é o vetor dos erros experimentais. Opcionalmente a matriz $M$ pode ser subdividida em $M=[X \vdots Z: G]$, onde $X$ é uma matriz com dimensões $(K J R \times K)$ de posto $K$ associada aos efeitos médios dos $K$ tratamentos (vetor de paràmetros $\theta$ ), $Z \in G$ são matrizes associadas aos efeitos de experimento e da interação tratamento $x$ experimento, respectivamente.

\subsubsection{A Função de Verossimilhança}

Para a determinação da função de verossimilhança é conveniente transformar as $J K R$ observações nos conjuntos $\left\{\bar{y}_{\ldots}\right\},\left\{\bar{y}_{i . .}-\bar{y}_{\ldots}\right\},\left\{\bar{y}_{. j .}-\bar{y}_{\ldots}\right\},\left\{\bar{y}_{i j .}-\right.$ $\left.\bar{y}_{i .}-\bar{y}_{j}+\bar{y}_{\ldots}\right\}$ e $\left\{y_{i j r}-\bar{y}_{i j}\right\}$, de onde segue que:

1. Os conjuntos $\left\{\bar{y}_{\ldots\}}\right\},\left\{\bar{y}_{i . .}-\bar{y}_{\ldots .}\right\},\left\{\bar{y}_{. j .}-\bar{y}_{\ldots}\right\},\left\{\bar{y}_{i j}-\bar{y}_{i . .}-\bar{y}_{. j}+\bar{y}_{\ldots .}\right\}$ e $\left\{y_{i j r}-\bar{y}_{i j .}\right\}$ são independentemente distribuídos uns dos outros, (Box \& TIAO, 1973);

2. $\bar{y}_{\ldots}=\frac{1}{K J B} \sum_{i=1}^{K} \sum_{j=1}^{J} \sum_{r=1}^{R} y_{i j r}=\bar{\theta}+\bar{\beta}+\bar{\gamma}_{.}+\bar{\epsilon}_{\ldots .}$. Assim sendo, $E\left[\bar{y}_{\ldots}\right]=\bar{\theta}$ e $\operatorname{Var}\left[\bar{y}_{\ldots} ..\right]$ $=\frac{1}{J} \sigma_{b}^{2}+\frac{1}{K J} \sigma_{g}^{2}+\frac{1}{K J R} \sigma_{e}^{2}=\frac{1}{K J R}\left(\sigma_{e}^{2}+R \sigma_{g}^{2}+K R \sigma_{b}^{2}\right)=\frac{1}{K J R} \sigma_{e g b}^{2}$. Portanto.

$$
\bar{y}_{\ldots} \sim N\left(\bar{\theta}, \frac{1}{K J R} \sigma_{e g b}^{2}\right)
$$

cuja função de densidade é dada por

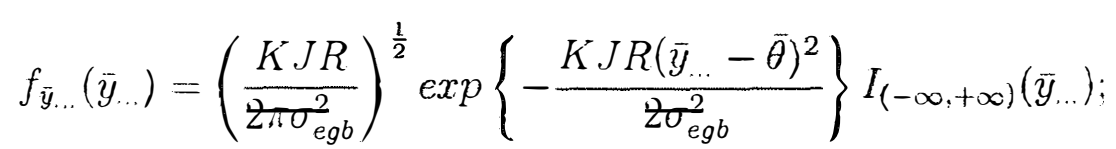

3. $\bar{y}_{i}-\bar{y}_{\ldots}=\theta_{i}-\bar{\theta}+\bar{\gamma}_{i,}-\bar{\gamma}_{.}+\bar{e}_{i . .}-\bar{e}_{\ldots .}$, desse modo, tem-se que $E\left[\bar{y}_{i . .}-\bar{y}_{\ldots}\right]=\theta_{i}-\bar{\theta}=$ $\phi_{i}$ e $\operatorname{Var}\left[\bar{y}_{i . .}-\bar{y}_{\ldots}\right]=E\left[\left(\bar{y}_{i . .}-\bar{y}_{\ldots} \ldots\right)-E\left(\bar{y}_{i . .}-\bar{y}_{\ldots}\right)\right]^{2}=\frac{(K-1)\left(\sigma_{s}^{2}+R \sigma_{2}^{2}\right)}{K}=\frac{(K-1)}{K R} \cdot \frac{1}{J R} \sigma_{e g}^{2}$, $(i=1, \ldots, K)$ e $\operatorname{Cov}\left[\left(\bar{y}_{i . .}-\bar{y}_{\ldots}\right),\left(\bar{y}_{s . .}-\bar{y}_{\ldots}\right)\right]=-\frac{1}{K} \cdot \frac{1}{J R} \sigma_{e g}^{2},(i \neq s)$. Logo, o vetor de contrastes dado por $\dot{\boldsymbol{\phi}}=D \hat{\boldsymbol{\theta}}=\left[\left(\bar{y}_{1 .,}-\bar{y}_{\ldots}\right),\left(\bar{y}_{2 . .}-\bar{y}_{\ldots}\right), \ldots,\left(\bar{y}_{(K-1)} .-\bar{y}_{\ldots}\right)\right]$ ' é distribuído como uma normal multivariada $(K-1)$-dimensional $N_{(K-1)}\left(\phi, \sigma_{e g}^{2} \Sigma\right)$, 
onde

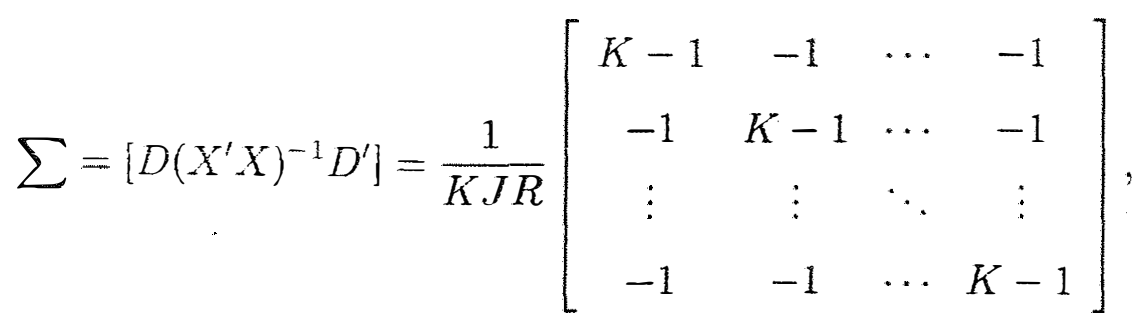

cujo determinante é dado por

$$
\left|\sum\right|=\frac{1}{K(J R)^{(K-1)}}
$$

e sua inversa com dimensão $(K-1) \times(K-1)$ é dada por

$$
\sum^{-1}=J R\left[\begin{array}{cccc}
2 & 1 & \cdots & 1 \\
1 & 2 & \cdots & 1 \\
\vdots & \vdots & \ddots & \vdots \\
1 & 1 & \cdots & 2
\end{array}\right]
$$

$D$ é uma matriz dos coeficientes de $K-1$ contrastes não redundantes, $\left(\bar{y}_{i . .}-\right.$ $\bar{y}_{\ldots}$ )'s, $i=1, \ldots, K-1$, com dimensões $(K-1) \times K$ e de posto $(K-1)$, isto é,

$$
D=\frac{1}{K}\left[\begin{array}{ccccc}
K-1 & -1 & \cdots & -1 & -1 \\
-1 & K-1 & \cdots & -1 & -1 \\
\vdots & \vdots & \ddots & \vdots & \vdots \\
-1 & -1 & \cdots & K-1 & -1
\end{array}\right] e
$$

$\left(X^{\prime} X\right)^{-1}$ tem dimensões $K \times K$ e posto $K$, ou seja,

$$
\left(X^{\prime} X\right)^{-1}=\frac{1}{J R}\left[\begin{array}{cccc}
1 & 0 & \cdots & 0 \\
0 & 1 & \cdots & 0 \\
\vdots & \vdots & \ddots & \vdots \\
0 & 0 & \cdots & 1
\end{array}\right]
$$

Dessa forma, a função de distribuição do conjunto de contrastes $\hat{\phi}$ é dada por

$$
\begin{aligned}
& f_{\hat{\phi}}(\hat{\phi})=\frac{\left|\sum\right|^{-\frac{1}{2}}}{\left(2 \pi \sigma_{e g}^{2}\right)^{\frac{k K}{2}} \ln p}\left\{-\frac{1}{2 \sigma_{e g}^{2}}(\phi-\hat{\phi})^{\prime} \sum^{-1}(\phi-\dot{\phi})\right\} \text {. } \\
& -\infty<\hat{\phi}_{i}<+\infty, \quad i=1,2, \ldots,(K-1)
\end{aligned}
$$


ou

$$
\begin{aligned}
f_{\dot{\phi}}(\hat{\phi})= & \sqrt{K}\left(\frac{J R}{2 \pi \sigma_{e g}^{2}}\right)^{\frac{K-1}{2}} \exp \left\{-\frac{1}{2 \sigma_{e g}^{2}}(\phi-\hat{\phi})^{\prime} \sum^{-1}(\phi-\hat{\phi})\right\} \\
& -\infty<\hat{\phi}_{i}<+\infty, \quad i=1,2, \ldots,(K-1)
\end{aligned}
$$

ou ainda

$$
\begin{aligned}
f_{\grave{\phi}}(\hat{\phi})= & \sqrt{K}\left(\frac{J R}{2 \pi \sigma_{e g}^{2}}\right)^{\frac{K-1}{2}} \exp \left\{-\frac{S(\phi)}{2 \sigma_{e g}^{2}}\right\} \\
& -\infty<\left(\bar{y}_{i . .}-\bar{y}_{\ldots}\right)=\hat{\phi}_{i}<+\infty, \quad i=1,2, \ldots,(K-1)
\end{aligned}
$$

onde

$$
S(\phi)=\nu_{\phi} m(\phi)=J R \sum_{i=1}^{K}\left[\phi_{i}-\left(\bar{y}_{i . .}-\bar{y}_{\ldots}\right)\right]^{2},
$$

segue que, sob $H_{0}: \boldsymbol{\phi}=\mathbf{0}$, (isto $e_{1} H_{0}: \theta_{1}=\theta_{2}=\ldots=\theta_{K}$ ), a estatística

$$
\frac{J R \sum_{i=1}^{K}\left(\bar{y}_{i . .}-\bar{y} \ldots\right)^{2}}{\sigma_{e g}^{2}}=\frac{\nu_{a} m_{a}}{\sigma_{e g}^{2}}=\frac{S_{a}}{\sigma_{e g}^{2}} \sim \chi_{\left(\nu_{a}\right)}^{2}, \quad \nu_{\phi}=\nu_{a}=K-1 .
$$

4. $\bar{y}_{. j}-\bar{y}_{\ldots}=\beta_{j}-\bar{\beta}_{.}+\bar{\gamma}_{j}-\bar{\gamma}_{.}+\bar{e}_{j .}-\bar{e}_{\ldots}$. Segue portanto que, $E\left[\bar{y}_{j .}-\bar{y}_{\ldots}\right]=0 \mathrm{e}$ $\operatorname{Var}\left[\bar{y}_{j .}-\bar{y}_{\ldots .}\right]=\frac{(J-1)}{J} \cdot \frac{1}{\underline{K} R}\left(\sigma_{e}^{2}+R \sigma_{g}^{2}+K R \sigma_{b}^{2}\right)=\frac{(J-1)}{J} \cdot \frac{1}{K^{L} R} \sigma_{e g b}^{2}$. Consequentemente,

$$
\begin{aligned}
& \left(\bar{y}_{, j}-\bar{y}_{\ldots}\right) \sim N\left(0, \frac{(J-1)}{J} \cdot \frac{1}{K R} \sigma_{e g b}^{2}\right) e \\
& \sum_{j=1}^{J}\left(\bar{y}_{. j .}-\bar{y}_{\ldots}\right) \sim N\left(0,(J-1) \cdot \frac{1}{K R} \sigma_{e g b}^{2}\right) .
\end{aligned}
$$

Assim sendo, $\sum_{j}\left(\bar{y}_{. j}-\bar{y}_{. .}\right)$pode ser imaginado como sendo proveniente de $(J-1)$ observaçōes independentes de uma distribuição normal $N\left(0, \frac{1}{K} \bar{R} \sigma_{\text {egb }}^{2}\right)$. E. portanto

$$
\frac{K R \sum_{j=1}^{J}\left(\bar{y}_{. j .}-\bar{y}_{. .}\right)^{2}}{\sigma_{e g b}^{2}}=\frac{\nu_{b} m_{b}}{\sigma_{e g b}^{2}}=\frac{S_{b}}{\sigma_{e g b}^{2}} \sim \chi_{\left(\nu_{b}\right)}^{2}, \quad \nu_{b}=J-1,
$$

cuja distribuição de probabilidade da estatística $S_{b}=\nu_{b} m_{b}$ é

$$
f_{S_{b}}\left(s_{b}\right)=\frac{\left(\nu_{b} m_{b}\right)^{\left(\frac{\nu_{b}}{2}-1\right)}}{\Gamma\left(\frac{\nu_{b}}{2}\right) 2^{\frac{\nu_{b}}{2}}}\left(\sigma_{e g b}^{2}\right)^{-\frac{\nu_{b}}{2}} \exp \left\{-\frac{\nu_{b} m_{b}}{2 \sigma_{e g b}^{2}}\right\} I_{(0, \infty)}\left(s_{a}\right)
$$


j. $\bar{y}_{i j .}-\bar{y}_{i . .}-\bar{y}_{. j .}+\bar{y}_{\ldots}=\gamma_{i j}-\bar{\gamma}_{i .}-\bar{\gamma}_{j}+\bar{\gamma}_{. .}+\bar{e}_{i j .}-\bar{e}_{i . .}-\bar{e}_{j .}+\bar{e}_{, \ldots} . \quad$ Dessa forma, tem-se que $E\left[\bar{y}_{i j .}-\bar{y}_{i . .}-\bar{y}_{. j}+\bar{y}_{\ldots}\right]=0$ e $\operatorname{Var}\left[\bar{y}_{i j .}-\bar{y}_{i . .}-\bar{y}_{. j .}+\bar{y}_{\ldots .}\right]=$ $\frac{(K-1)}{K} \cdot \frac{(J-1)}{J} \cdot \frac{1}{R}\left(\sigma_{e}^{2}+R \sigma_{g}^{2}\right)=\frac{(K-1)}{K} \cdot \frac{(J-1)}{J} \cdot \frac{1}{R} \sigma_{e g}^{2}$. Consequentemente

$$
\begin{aligned}
& \left(\bar{y}_{i j .}-\bar{y}_{i . .}-\bar{y}_{. j .}+\bar{y}_{\ldots}\right) \sim N\left(0, \frac{(K-1)}{K} \cdot \frac{(J-1)}{J} \cdot \frac{1}{R} \sigma_{e g}^{2}\right) e \\
& \sum_{i=1}^{K} \sum_{j=1}^{J}\left(\bar{y}_{i j .}-\bar{y}_{i . .}-\bar{y}_{. j .}+\bar{y}_{\ldots .}\right) \sim N\left(0,(K-1)(J-1) \frac{1}{R} \sigma_{e g}^{2}\right)
\end{aligned}
$$

De modo análogo, $\sum_{i=1}^{K} \sum_{j=1}^{J}\left(\bar{y}_{i j .}-\bar{y}_{i . .}-\bar{y}_{. j .}+\bar{y}_{\ldots}\right)$ pode ser imaginada como sendo proveniente de $\nu_{g}=(K-1)(J-1)$ observações independentes de uma distribuição normal $N\left(0, \frac{1}{R} \sigma_{e g}^{2}\right)$. E, portanto,

$$
\frac{R \sum_{i=1}^{K} \sum_{j=1}^{J}\left(\bar{y}_{i j .}-\bar{y}_{i . .}-\bar{y}_{. j .}+\bar{y}_{\ldots . .}\right)^{2}}{\sigma_{e g}^{2}}=\frac{\nu_{g} m_{g}}{\sigma_{e g}^{2}}=\frac{S_{g}}{\sigma_{e g}^{2}} \sim \chi_{\left(\nu_{g}\right)}^{2},
$$

cuja função de densidade associada a variável $S_{g}=\nu_{g} m_{g}$, é

$$
f_{S_{g}}\left(s_{g}\right)=\frac{\left(\nu_{g} m_{g}\right)^{\left(\frac{\nu_{g}}{2}-1\right)}}{\Gamma\left(\frac{\nu_{g}}{2}\right) 2^{\frac{\nu_{g}}{2}}}\left(\sigma_{e g}^{2}\right)^{-\frac{\nu_{g}}{2}} \exp \left\{-\frac{\nu_{g} m_{g}}{2 \sigma_{e g}^{2}}\right\} I_{(0, \infty)}\left(s_{g}\right)
$$

6. Finalmente, $y_{i j r}-\bar{y}_{i j .}=e_{i j r}-\bar{e}_{i j .}, E\left[y_{i j r}-\bar{y}_{i j}\right]=0$ e $\operatorname{Var}\left[y_{i j r}-\bar{y}_{i j}\right]=$ $\left(\underline{R}-\frac{1}{R} \sigma_{e}^{2}\right.$. Desse modo, $y_{i j r}-\bar{y}_{i j .} \sim N\left(0, \frac{(R-1)}{R} \sigma_{e}^{2}\right)$ e $\sum_{i=1}^{K} \sum_{j=1}^{J} \sum_{r=1}^{R}\left(y_{i j r}-\bar{y}_{i j}\right) \sim$ $N\left(0, K J(R-1) \sigma_{e}^{2}\right)$ a qual pode ser imaginada como sendo proveniente de $\nu_{e}$ $=K J(R-1)$ observações de uma distribuição normal $N\left(0, \sigma_{e}^{2}\right)$. Consequentemente,

$$
\frac{\sum_{i=1}^{K} \sum_{j=1}^{J} \sum_{r=1}^{R}\left(y_{i j r}-\bar{y}_{i} j .\right)^{2}}{\sigma_{e}^{2}}=\frac{\nu_{e} m_{e}}{\sigma_{e}^{2}}=\frac{S_{e}}{\sigma_{e}^{2}} \sim \chi_{\left(\nu_{e}\right)}^{2}
$$

cuja função de densidade da variável aleatória $S_{e}$ é dada por

$$
f_{S_{e}}\left(s_{e}\right)=\frac{\left(\nu_{e} m_{e}\right)^{\left(\frac{\nu_{e}}{2}-1\right)}}{\Gamma\left(\frac{\nu_{e}}{2}\right) 2^{\frac{\nu_{e}}{2}}}\left(\sigma_{e}^{2}\right)^{-\frac{\nu_{e}}{2}} \exp \left\{-\frac{\nu_{e} m_{e}}{2 \sigma_{e}^{2}}\right\} I_{(0, \infty)}\left(s_{e}\right) .
$$

A tabela 2 apresenta um resumo dos resultados obtidos nos ítens de 1 a 6 , a qual corresponde ao quadro da análise de variância com as esperanças dos quadrados médios. 
Tabela 2: Quadro da análise de variància com as esperanças dos quadrados médios para o modelo misto com dois fatores com interação.

\begin{tabular}{lcccl}
\hline F. Variaçào & G.1. & S.Q. & Q.M. & \multicolumn{1}{c}{ E[Q.M.] } \\
\hline Média Geral & 1 & $S_{\mu}$ & $S_{\mu}$ & $\sigma_{e}^{2}+R \sigma_{g}^{2}+K R \sigma_{b}^{2}$ \\
Trat.(T) & $\nu_{\phi}$ & $S(\phi)$ & $m(\phi)=\frac{S(\phi)}{\nu_{p}}$ & $\sigma_{e g}^{2}=\sigma_{e}^{2}+R \sigma_{g}^{2}$ \\
Ensaio(E) & $\nu_{b}$ & $S_{b}=\nu_{b} m_{b}$ & $m_{b}=\frac{S_{b}}{\nu_{b}}$ & $\sigma_{e g b}^{2}=\sigma_{e}^{2}+R \sigma_{g}^{2}+K R \sigma_{b}^{2}$ \\
Int.E $\times \mathrm{T}$ & $\nu_{g}$ & $S_{g}=\nu_{g} m_{g}$ & $m_{g}=\frac{S_{g}}{\nu_{g}}$ & $\sigma_{e g}^{2}=\sigma_{e}^{2}+R \sigma_{g}^{2}$ \\
Resíduo & $\nu_{e}$ & $S_{e}=\nu_{e} m_{e}$ & $m_{e}=\frac{S_{e}}{\nu_{e}}$ & $\sigma_{e}^{2}$ \\
\hline Total & $K J R$ & $S_{t}$ & - & -
\end{tabular}

onde

$$
\begin{aligned}
& S_{t}= \sum_{i j r}\left(y_{i j r}-\mu\right)^{2} \\
&= \sum_{i j r}\left[\left(\bar{y}_{\ldots}-\mu\right)+\left(\bar{y}_{i . .}-\bar{y}_{\ldots}\right)+\left(\bar{y}_{j .}-\bar{y}_{\ldots}\right)+\left(\bar{y}_{i j .}-\bar{y}_{i . .}-\bar{y}_{j .}++\bar{y}_{\ldots}\right)\right. \\
&\left.+\left(y_{i j r}-\bar{y}_{i j .}\right)\right]^{2} \\
&= K J R\left(\bar{y}_{\ldots}-\mu\right)^{2}+J R \sum_{i=1}^{K}\left(\bar{y}_{i . .}-\bar{y}_{\ldots}\right)^{2}+K R \sum_{j=1}^{J}\left(\bar{y}_{. j .}-\bar{y}_{\ldots}\right)^{2} \\
& \quad+R \sum_{i=1}^{K} \sum_{j=1}^{J}\left(\bar{y}_{i j .}-\bar{y}_{i . .}-\bar{y}_{j .}+\bar{y}_{\ldots}\right)^{2}+\sum_{i=1}^{K} \sum_{j=1}^{J} \sum_{r=1}^{R}\left(y_{i j r}-\bar{y}_{i j .}\right)^{2} \\
&=S_{\mu}+S(\phi=\mathbf{0})+S_{b}+S_{g}+S_{e}=S_{\mu}+\nu_{\phi} m(\dot{\phi})+\nu_{b} m_{b}+\nu_{g} m_{g}+\nu_{e} m_{t}(78)
\end{aligned}
$$

Sendo $S_{\mu}, S(\phi), S_{b}, S_{g}$ e $S_{e}$ variáveis aleatórias independentemente distribuidas, a função de verossimilhança pode ser obtida pelo produto das densidades em (62), (70), (73), (75) e (77), a saber,

$$
\begin{aligned}
& \ell\left(\vec{\theta}, \phi, \sigma_{e}^{2}, \sigma_{e g}^{2}, \sigma_{e g b}^{2} \mid y\right) \\
& \propto\left(\sigma_{e}^{2}\right)^{-\frac{\nu_{e}}{2}}\left(\sigma_{e g}^{2}\right)^{-\frac{1}{2}\left(\nu_{\phi}+\nu_{g}\right)}\left(\sigma_{e g b}^{2}\right)^{-\frac{1}{2}\left(\nu_{t}+1\right)} \exp \left\{-\frac{1}{2}\left[\frac{\nu_{e} m_{e}}{\sigma_{e}^{2}}+\frac{\nu_{g} m_{g}+S(\phi)}{\sigma_{e g}^{2}}+\frac{\nu_{b} m_{b}+S_{\mu}}{\sigma_{e g b}^{2}}\right]\right\} \\
& -\infty<\bar{\theta}<+\infty, \quad-\infty<\phi_{i}<+\infty, \quad \sigma_{e g b}^{2}>\sigma_{e g}^{2}>\sigma_{e}^{2}>0, \quad i=1, \ldots, K-1
\end{aligned}
$$


Observa-se que as quantidades que aparecem na funçào de verossimilhança, correspondem àquelas convenientemente organizadas no quadro da análise da variància (tabela 2 ).

\subsubsection{A Distribuição a priori de $\left(\bar{\theta}, \phi, \sigma_{e}^{2}, \sigma_{e g}^{2}, \sigma_{e g b}^{2}\right)$}

Admitindo um argumento similar ao adotado nas análises individuais. empregou-se, também na análise conjunta, uma distribuição a priori de referència não informativa, isto é,

$$
p\left(\bar{\theta}, \phi, \sigma_{e}^{2}, \sigma_{e g}^{2}, \sigma_{e g b}^{2}\right) \propto \frac{1}{\sigma_{e}^{2} \sigma_{e g}^{2} \sigma_{e g b}^{2}}
$$

com a restrição

$$
C: \sigma_{e g b}^{2}>\sigma_{e g}^{2}>\sigma_{e}^{2}>0
$$

A restrição $C: \sigma_{e g b}^{2}>\sigma_{e g}^{2}>\sigma_{e}^{2}>0$ é imposta devido ao fato de não ser plausível se admitir a priori que se tenha algum componente de variância negatiro.

\subsubsection{A Distribuição a Posteriori de $\left(\bar{\theta}, \phi, \sigma_{e}^{2}, \sigma_{e g}^{2}, \sigma_{e g b}^{2}\right)$}

Agora. combinando a verossimilhança em (79) com a distribuição a priori em (80), tem-se a distribuição a posteriori, ou seja,

$$
\begin{aligned}
& p\left(\bar{\theta}, \phi, \sigma_{e}^{2}, \sigma_{e g}^{2}, \sigma_{e g b}^{2} \mid \boldsymbol{y}\right)=W_{c}\left(\sigma_{e}^{2}\right)^{-\left(\frac{\nu_{e}}{2}-1\right)}\left(\sigma_{e g}^{2}\right)^{-\left(\frac{\nu_{g}+\nu_{\phi}}{2}-1\right)}\left(\sigma_{e g b}^{2}\right)^{-\left(\frac{\nu_{t}}{2}+1\right)-\frac{1}{2}} \\
& \quad \times \exp \left\{-\frac{1}{2}\left[\frac{\nu_{e} m_{e}}{\sigma_{e}^{2}}+\frac{\nu_{g} m_{g}+S(\phi)}{\sigma_{e g}^{2}}+\frac{K J R(\bar{y} \ldots-\bar{\theta})^{2}}{\sigma_{e g b}^{2}}\right]\right\}, \quad-\infty<\bar{\theta}<+\infty \\
& \quad-\infty<\phi_{i}<+\infty, \quad \sigma_{e g b}^{2}>\sigma_{e g}^{2}>\sigma_{e}^{2}>0, \quad i=1, \ldots, K-1
\end{aligned}
$$

onde

$$
H_{c}^{-1}=\int_{-\infty}^{+\infty} \int_{\phi} \int_{0}^{\infty} \int_{\sigma_{\varepsilon}^{2}}^{\infty} \int_{\sigma_{\varepsilon g}^{2}}^{\infty} p\left(\bar{\theta}, \phi, \sigma_{e}^{2}, \sigma_{e g}^{2}, \sigma_{e g b}^{2} \mid \boldsymbol{y}\right) d \sigma_{e g b}^{2} d \sigma_{e g}^{2} d \sigma_{e}^{2} d \phi d \bar{\theta}
$$


Para encontrar o valor de $W_{c}^{-1}$ empregou-se a identidade conhecida por integral de Dirichlet, a saber.

$$
\begin{aligned}
\operatorname{Pr} & \left\{\frac{\chi_{2 p_{1}}^{2}}{\chi_{2 p_{2}}^{2}}>\frac{a_{1}}{a_{2}} ; \frac{\chi_{2 p_{2}}^{2}}{\chi_{2 p_{3}}^{2}}>\frac{a_{2}}{a_{3}}\right\} \\
= & c_{1} c_{2} \prod_{i=1}^{3} \frac{a_{i}^{p_{i}}}{\Gamma\left(p_{i}\right)} \int_{0}^{\infty} \int_{0}^{\infty} \int_{0}^{\infty} x_{1}^{-\left(p_{1}+1\right)}\left(x_{1}+c_{1} x_{2}\right)^{-\left(p_{2}+1\right)}\left(x_{1}+c_{1} x_{2}+c_{2} x_{3}\right)^{-\left(p_{3}+1\right)} \\
& \quad \exp \left\{-\left(\frac{a_{1}}{x_{1}}+\frac{a_{2}}{x_{1}+c_{1} x_{2}}+\frac{a_{3}}{x_{1}+c_{1} x_{2}+c_{2} x_{3}}\right)\right\} d x_{1} d x_{2} d x_{3},
\end{aligned}
$$

onde $p_{i}>0, a_{i}>0, i=1,2,3, c_{1}>0, c_{2}>0 \mathrm{e}\left(\chi_{2 p_{1}}^{2}, \chi_{2 p_{2}}^{2}, \chi_{2 p_{3}}^{2}\right)$ são variáveis aleatórias distribuidas independentemente umas das outras como qui-quadrado com $\left(2 p_{1}, 2 p_{2}, 2 p_{3}\right)$ graus de liberdade, respectivamente. Usando a identidade (84), após algumas operações algébrica, chegou-se ao seguinte resultado,

$$
W_{c}^{-1}=\frac{1}{K}\left(\frac{2 \pi}{J R}\right)^{\frac{\nu_{\phi}+1}{2}} \frac{\Gamma\left(\frac{\nu_{e}}{2}\right) \Gamma\left(\frac{\nu_{g}}{2}\right) \Gamma\left(\frac{\nu_{b}}{2}\right) 2^{\frac{\nu_{g}+\nu_{g}+\nu_{g}}{2}}}{\left(\nu_{e} m_{e}\right)^{\frac{\nu_{e}}{2}}\left(\nu_{g} m_{g}\right)^{\frac{\nu_{g}}{2}}\left(\nu_{b} m_{b}\right)^{\frac{\nu_{b}}{2}}} \operatorname{Pr}\left\{\frac{\chi_{\nu_{g}}^{2}}{\chi_{\nu_{g}}^{2}}>\frac{\nu_{e} m_{e}}{\nu_{g} m_{g}} ; \frac{\chi_{\nu_{g}}^{2}}{\chi_{\nu_{b}}^{2}}>\frac{\nu_{g} m_{g}}{\nu_{b} m_{b}}\right\}
$$

onde $\left(\chi_{\nu_{s}}^{2}, \chi_{\nu_{g}}^{2}, \chi_{\nu_{b}}^{2}\right)$ são variáveis aleatórias independentes, distribuídas como quiquadrado com $\left(\nu_{e}, \nu_{g}, \nu_{b}\right)$ graus de liberdade, respectivamente.

A partir da identidade dada em (84), pode ser verificado que

$$
\begin{aligned}
\operatorname{Pr}\{ & \left.\frac{\chi_{\nu_{e}}^{2}}{\chi_{\nu_{g}}^{2}}>\frac{\nu_{e} m_{e}}{\nu_{g} m_{g}} ; \frac{\chi_{\nu_{g}}^{2}}{\chi_{\nu_{b}}^{2}}>\frac{\nu_{g} m_{g}}{\nu_{b} m_{b}}\right\} \\
= & I_{\overline{\nu_{b} m_{b}+\nu_{b} m_{g}}}\left(\frac{\nu_{b}}{2}, \frac{\nu_{g}}{2}\right)-\left[B\left(\frac{\nu_{e}}{2}, \frac{\nu_{g}}{2}\right) B\left(\frac{\nu_{e}+\nu_{g}}{2}, \frac{\nu_{b}}{2}\right)\right]^{-1} \\
& \times \int_{0}^{\nu_{e} m_{\varepsilon} / \nu_{g} m_{g}} \int_{0}^{\nu_{b} m_{b} / \nu_{g} m_{g}} t_{1}^{\left(\frac{\nu_{e}}{2}-1\right)} t_{2}^{\left(\frac{\nu_{b}}{2}-1\right)}\left(1+t_{1}+t_{2}\right)^{-\left(\frac{\nu_{e}+\nu_{g}+\nu_{b}}{2}\right)} d t_{2} d t_{1},
\end{aligned}
$$

onde $I_{x}(p, q)=\frac{\Gamma(p+q)}{\Gamma(p) \Gamma(q)} \int_{0}^{x} u^{p-1}(1-u)^{q-1} d u$ (a integral de uma beta), $x=\frac{\nu_{b} m_{b}}{\nu_{b} m_{b}+\nu_{g} m_{g}}$ $\operatorname{B}(p, q)=\frac{\Gamma(p) \Gamma(q)}{\Gamma(p+q)}$.

Substituindo-se (85) em (82), obtém-se a densidade conjunta a posteriori de $\left(\bar{\theta}, \phi, \sigma_{e}^{2}, \sigma_{e g}^{2}, \sigma_{e g b}^{2}\right)$, isto é, 


$$
\begin{aligned}
& p\left(\bar{\theta}, \phi, \sigma_{e}^{2}, \sigma_{e g}^{2}, \sigma_{e g b}^{2} \mid y\right)=\left(\frac{K J R}{2 \pi \sigma_{e g b}^{2}}\right)^{\frac{1}{2}} \exp \left\{-\frac{(\bar{\theta}-\bar{y} \ldots)^{2}}{2 \sigma_{e g b}^{2}}\right\} \times \\
& \sqrt{K}\left(\frac{J R}{2 \pi \sigma_{e g}^{2}}\right)^{\frac{\nu_{\phi}}{2}} \exp \left\{-\frac{(\phi-\hat{\phi})^{\prime} \sum^{-1}(\phi-\hat{\phi})}{2 \sigma_{e g}^{2}}\right\} \times \\
& \frac{\left(\nu_{e} m_{e}\right)^{-1} p\left(\chi_{\nu_{e}}^{-2}=\frac{\sigma_{e}^{2}}{\nu_{\varepsilon} m_{e}}\right)\left(\nu_{g} m_{g}\right)^{-1} p\left(\chi_{\nu_{g}}^{-2}=\frac{\sigma_{e g}^{2}}{\nu_{g} m_{g}}\right)\left(\nu_{b} m_{b}\right)^{-1} p\left(\chi_{\nu_{t}}^{-2}=\frac{\sigma_{e g b}^{2}}{\nu_{b} m_{b}}\right)}{\operatorname{Pr}\left\{\frac{\chi_{\nu_{e}}^{2}}{\chi_{\nu_{g}}^{2}}>\frac{\nu_{e} m_{e}}{\nu_{g} m_{g}} ; \frac{\chi_{\nu_{g}}^{2}}{\chi_{\nu_{b}}^{2}}>\frac{\nu_{g} m_{g}}{\nu_{b} m_{b}}\right\}} \\
& -\infty<\bar{\theta}<+\infty, \quad-\infty<\phi_{i}<+\infty, \quad \sigma_{e g b}^{2}>\sigma_{e g}^{2}>\sigma_{e}^{2}>0, \quad i=1, \ldots, K-1,
\end{aligned}
$$

onde $p\left(\chi_{\nu}^{-2}=x\right)$ é a densidade de uma distribuição de qui-quadrado inversa com $\nu$ graus de liberdade avaliada no ponto $x$.

A densidade conjunta a posteriori dada em (87), pode ser expressa da seguinte forma,

$$
\begin{aligned}
& p\left(\bar{\theta}, \boldsymbol{\phi}, \sigma_{e}^{2}, \sigma_{e g}^{2}, \sigma_{e g b}^{2} \mid \boldsymbol{y}\right)=p\left(\bar{\theta} \mid \sigma_{e g b}^{2}, \boldsymbol{y}\right) p\left(\boldsymbol{\phi} \mid \sigma_{e g}^{2}, \boldsymbol{y}\right) p\left(\sigma_{e}^{2}, \sigma_{e g}^{2}, \sigma_{e g b}^{2} \mid \boldsymbol{y}\right) \\
& -\infty<\bar{\theta}<+\infty, \quad-\infty<\phi_{i}<+\infty, \quad \sigma_{e g b}^{2}>\sigma_{e g}^{2}>\sigma_{e}^{2}>0, \quad i=1, \ldots, K-1,
\end{aligned}
$$

Na expressão (87), observa-se que, (i) a distribuição condicional de $\bar{\theta}$ dado $\sigma_{e g b}^{2}, p\left(\bar{\theta} \mid \sigma_{e g b}^{2}, \boldsymbol{y}\right)$, é normal univariada $N\left(\bar{y} \ldots ; \frac{1}{\bar{K}} \bar{J} \bar{R}_{e g b}^{2}\right) ;$ (ii) a distribuição condicional de $\phi$ dado $\sigma_{e g}^{2}, p\left(\phi \mid \sigma_{e g}^{2}, \boldsymbol{y}\right)$, é normal multivariada (K-1)-dimensional $N_{(K-1)}\left(\hat{\phi} ; \sigma_{e g}^{2} \sum\right)$, onde $\sum$ é dada em $(63)$ e $\hat{\phi}=\left[\left(\bar{y}_{1 . .}-\bar{y}_{\ldots}\right),\left(\bar{y}_{2 . .}-\bar{y}_{\ldots}\right), \ldots,\left(\bar{y}_{(K-1) . .}-\right.\right.$ $\bar{y} .).]^{\prime}$ é um vetor de contrastes já mencionado anteriormente e (iii) a densidade marginal a posteriori de $\left(\sigma_{e}^{2}, \sigma_{e g}^{2}, \sigma_{e g b}^{2}\right)$ é,

$$
\begin{aligned}
& p\left(\sigma_{e}^{2}, \sigma_{e g}^{2}, \sigma_{e g b}^{2} \mid \boldsymbol{y}\right) \\
& =\frac{\left(\nu_{e} m_{e}\right)^{-1} p\left(\chi_{\nu_{e}}^{-2}=\frac{\sigma_{s}^{2}}{\nu_{e} m_{e}}\right)\left(\nu_{g} m_{g}\right)^{-1} p\left(\chi_{\nu_{g}}^{-2}=\frac{\sigma_{e g}^{2}}{\nu_{g} m_{g}}\right)\left(\nu_{b} m_{b}\right)^{-1} p\left(\chi_{\nu_{b}}^{-2}=\frac{\sigma_{e g b}^{2}}{\nu_{b} m_{b}}\right)}{\operatorname{Pr}\left\{\frac{\chi_{\nu_{e}}^{2}}{\chi_{\nu_{g}}^{2}}>\frac{\nu_{e} m_{e}}{\nu_{g} m_{g}} ; \frac{\chi_{\nu_{g}}^{2}}{\chi_{\nu_{b}}^{2}}>\frac{\nu_{g} m_{g}}{\nu_{b} m_{b}}\right\}} \\
& \sigma_{e g b}^{2}>\sigma_{e g}^{2}>\sigma_{e}^{2}>0
\end{aligned}
$$

onde $\left(\chi_{\nu_{e}}^{2}, \chi_{\nu_{g}}^{2}, \chi_{\nu_{t}}^{2}\right)$ são variáveis aleatórias independentes distribuídas como quiquadrado com $\left(\nu_{e}, \nu_{g}, \nu_{b}\right)$ graus de liberdade, respectivamente. Inferèncias sobre os 
componentes compostos da variância, $\left(\sigma_{e}^{2}, \sigma_{e g}^{2}, \sigma_{e g b}^{2}\right)$, podem ser feitas empregando-se a distribuição a posteriori em (89).

\subsubsection{Distribuição Marginal a Posteriori dos Componentes da Variância} $\left(\sigma_{e}^{2}, \sigma_{g}^{2}, \sigma_{b}^{2}\right)$

Para se obter a distribuição marginal a posteriori dos componentes da variància $\left(\sigma_{e}^{2}, \sigma_{g}^{2}, \sigma_{b}^{2}\right)$, basta multiplicar $p\left(\sigma_{e}^{2}, \sigma_{e g}^{2}, \sigma_{e g b}^{2} \mid \boldsymbol{y}\right)$ pelo jacobiano da transformação $|\mathcal{J}|$, isto é,

$$
p\left(\sigma_{e}^{2}, \sigma_{g}^{2}, \sigma_{b}^{2} \mid \boldsymbol{y}\right)=p\left(\sigma_{e}^{2}, \sigma_{e g}^{2}, \sigma_{e g b}^{2} \mid \boldsymbol{y}\right)|\mathcal{J}|
$$

onde

$$
|\mathcal{J}|=\left|\begin{array}{ccc}
\frac{\partial \sigma_{s}^{2}}{\partial \sigma_{e}^{2}} & \frac{\partial \sigma_{s}^{2}}{\partial \sigma_{g}^{2}} & \frac{\partial \sigma_{s}^{2}}{\partial \sigma_{b}^{2}} \\
\frac{\partial \sigma_{s q}^{2}}{\partial \sigma_{\varepsilon}^{2}} & \frac{\partial \sigma_{s g}^{2}}{\partial \sigma_{g}^{2}} & \frac{\partial \sigma_{s g}^{2}}{\partial \sigma_{b}^{2}} \\
\frac{\partial \sigma_{s g b}^{2}}{\partial \sigma_{s}^{2}} & \frac{\partial \sigma_{s g b}^{2}}{\partial \sigma_{g}^{2}} & \frac{\partial \sigma_{e g b}^{2}}{\partial \sigma_{b}^{2}}
\end{array}\right|=\left|\begin{array}{ccc}
1 & 0 & 0 \\
1 & R & 0 \\
1 & R & K R
\end{array}\right|=\mathrm{KR}^{2}
$$

substituindo-se este resultado em (89) e lembrando-se que $\sigma_{e g}^{2}=\sigma_{e}^{2}+R \sigma_{g}^{2}$ e $\sigma_{e g b}^{2}=$ $\sigma_{e}^{2}+R \sigma_{g}^{2}+K R \sigma_{b}^{2}$, a densidade a posteriori dos componentes da variância $\left(\sigma_{e}^{2}, \sigma_{g}^{2}, \sigma_{b}^{2}\right)$ fica,

$$
\begin{aligned}
& p\left(\sigma_{e}^{2}, \sigma_{g}^{2}, \sigma_{b}^{2} \mid \boldsymbol{y}\right) \\
& =K R^{2} \frac{\left(\nu_{e} m_{e}\right)^{-1} p\left(\chi_{\nu_{e}}^{-2}=\frac{\sigma_{e}^{2}}{\nu_{e} m_{e}}\right)\left(\nu_{g} m_{g}\right)^{-1} p\left(\chi_{\nu_{g}}^{-2}=\frac{\sigma_{e}^{2}+R \sigma_{g}^{2}}{\nu_{g} m_{g}}\right)\left(\nu_{b} m_{b}\right)^{-1} p\left(\chi_{\nu_{b}}^{-2}=\frac{\sigma_{e}^{2}+R \sigma_{g}^{2}+K R \sigma_{b}^{2}}{\nu_{b} m_{b}}\right)}{\operatorname{Pr}\left\{\frac{\chi_{\nu_{e}}^{2}}{\chi_{\nu_{g}}^{2}}>\frac{\nu_{e} m_{e}}{\nu_{g} m_{g}} ; \frac{\chi_{\nu_{g}}^{2}}{\chi_{\nu_{b}}^{2}}>\frac{\nu_{g} m_{g}}{\nu_{b} m_{b}}\right\}} \\
& \sigma_{e}^{2}>0, \quad \sigma_{g}^{2}>0, \quad \sigma_{b}^{2}>0 .
\end{aligned}
$$

Inferèncias conjunta sobre os componentes da variância $\left(\sigma_{\epsilon}^{2}, \sigma_{g}^{2}, \sigma_{b}^{2}\right)$, podem ser feitas usando a densidade a posteriori $p\left(\sigma_{e}^{2}, \sigma_{g}^{2}, \sigma_{b}^{2} \mid \boldsymbol{y}\right)$ dada em (92).

\subsubsection{Distribuição Marginal a Posteriori de $\phi$}

Para se obter a distribuição marginal a posteriori de $\phi$, é preciso integrar $p\left(\bar{\theta}, \phi, \sigma_{e}^{2}, \sigma_{e g}^{2}, \sigma_{e g b}^{2} \mid \boldsymbol{y}\right)$ em relação a $\left(\bar{\theta}, \sigma_{e}^{2}, \sigma_{e g}^{2}, \sigma_{e g b}^{2}\right)$, ou seja, 


$$
\begin{aligned}
p(\phi \mid y)= & \int_{0}^{\infty} \int_{\sigma_{\varepsilon}^{2}}^{\infty} \int_{\sigma_{\varepsilon g}}^{\infty} \int_{-\infty}^{+\infty} p\left(\vec{\theta}, \phi, \sigma_{e}^{2}, \sigma_{e g}^{2}, \sigma_{e g b}^{2} \mid y\right) d \vec{\theta} d \sigma_{e g b}^{2} d \sigma_{e g}^{2} d \sigma_{e}^{2} \\
& -\infty<\phi_{i}<+\infty, \quad i=1,2, \ldots, K-1
\end{aligned}
$$

a qual resulta em

$$
\begin{aligned}
p(\phi \mid y)= & W_{c}\left(\frac{2 \pi}{K J R}\right)^{\frac{1}{2}} \frac{\Gamma\left(\frac{\nu_{e}}{2}\right) \Gamma\left(\frac{\nu_{g}+\nu_{\rho}}{2}\right) \Gamma\left(\frac{\nu_{b}}{2}\right) 2^{\frac{\nu_{e}+\nu_{g}+\nu_{\phi}+\nu_{b}}{2}}}{\left(\nu_{e} m_{e}\right)^{\frac{\nu_{g}}{2}}\left[\nu_{g}+S(\phi)\right]^{\frac{\nu_{g}+\nu_{\phi}}{2}}\left(\nu_{b} m_{b}\right)^{\frac{\nu_{b}}{2}}} \\
& \times \operatorname{Pr}\left\{\frac{\chi_{\nu_{e}}^{2}}{\chi_{\left(\nu_{g}+\nu_{\phi}\right)}^{2}}>\frac{\nu_{e} m_{e}}{\nu_{g} m_{g}+S(\phi)}: \frac{\chi_{\left(\nu_{g}+\nu_{\phi}\right)}^{2}}{\chi_{\nu_{b}}^{2}}>\frac{\nu_{g} m_{g}+S(\phi)}{\nu_{b} m_{b}}\right\}
\end{aligned}
$$

onde $S(\phi)=(\phi-\hat{\phi})^{\prime} \sum^{-1}(\phi-\hat{\phi})=J R \sum_{i=1}^{K}\left[\phi_{i}-\left(\bar{y}_{i . .}-\bar{y}_{\ldots}\right)\right]^{2}$.

Substituindo-se o valor de $W_{c}$ obtido em (84) e fazendo algumas operaçòes algébricas encontra-se a distribuiçào marginal a posteriori de $\phi$, a saber,

$$
\begin{aligned}
p(\phi \mid y)= & \frac{\left|\sum\right|^{-\frac{1}{2}}\left(m_{g}\right)^{-\frac{\nu_{\phi}}{2}} \cdot \Gamma\left(\frac{\nu_{g}+\nu_{\phi}}{2}\right)}{\left[\Gamma\left(\frac{1}{2}\right)\right]^{\nu_{j}} \Gamma\left(\frac{\nu_{q}}{2}\right)\left(\nu_{g}\right)^{\frac{\nu_{g}}{2}}}\left[1+\frac{(\phi-\dot{\phi})^{\prime} \sum^{-1}(\phi-\dot{\phi})}{\nu_{g} m_{g}}\right]^{-\frac{\nu_{g}+\nu_{\phi}}{2}} g(\phi), \\
& -\infty<\phi_{i}<+\infty, \quad i=1,2, \ldots, K-1,
\end{aligned}
$$

onde

$$
g(\phi)=\frac{\operatorname{Pr}\left\{\frac{\chi_{\nu_{e}}^{2}}{\chi_{\left(\nu_{g}+\nu_{\phi}\right)}^{2}}>\frac{\nu_{s} m_{s}}{\nu_{g} m_{g}+S(\phi)} ; \frac{\chi_{\left(\nu_{g}+\nu_{b}\right)}^{2}}{\chi_{\nu_{b}}^{2}}>\frac{\nu_{g} m_{g}+S(\phi)}{\nu_{b} m_{b}}\right\}}{\operatorname{Pr}\left\{\frac{\chi_{\nu_{s}}^{2}}{\chi_{\nu_{g}}^{2}}>\frac{\nu_{s} m_{e}}{\nu_{g} m_{g}} ; \frac{\chi_{\nu_{g}}^{2}}{\chi_{\nu_{b}}^{2}}>\frac{\nu_{g} m_{g}}{\nu_{b} m_{b}}\right\}}
$$

$\grave{\phi} \cdot \sum \cdot\left|\sum\right| \cdot \Sigma^{-1}$ são de acordo como já definidos na seção $(3.3 .2)$ e $\left(\chi_{\nu_{e}}^{2}, \chi_{\nu_{b}}^{2}, \chi_{\left(\nu_{b}+\nu_{3}\right)}^{2}\right)$ sào variáveis aleatórias independentes, com distribuição de qui-quadrado, associadas $a\left(\nu_{e}, \nu_{b}, \nu_{0}+\nu_{g}\right)$ graus de liberdade, respectivamente.

Observa-se que o primeiro fator em (95) é a densidade de uma t-Student multivariada $(K-1)$-dimensional, $t_{(K-1)}\left(\hat{\phi}, m_{g} \sum, \nu_{g}\right)$, a qual será a distriiniçào a posteriori de $\phi$ se a restrição $C: \sigma_{e g b}^{2}>\sigma_{e g}^{2}>\sigma_{e}^{2}$ for ignorada. O segundo fat $g(\phi)$ em (96) é chamado de fator modificador e representa o efeito da restrição $\zeta: \sigma_{e g o}^{2}>\sigma_{e g}^{2}>\sigma_{e}^{2}$. Sendo $g(\phi)$ uma função apenas de $S(\phi)$, segue que o centro da distribuição em (95) está em $\dot{\phi}$ e os contornos de $\phi$ são elipsoidais. É fácil ver que 
$\hat{\phi}$ é o vetor das médias a posteriori de $\phi$ e é também a única moda da distribuição. Segundo BOX \& TiAO(1973), estas propriedades são muito semelhantes às da distribuição de $\phi$ nas análises quando se considera um modelo misto com dois fatores sem interação. No entanto, $g(\phi)$ não torna a distribuição $p(\phi \mid y)$ no modelo com dois fatores com interação mais monotonicamente decrescente da quantidade $S(\phi)$ uma vez que $g(\phi)$ não torna a distribuição de $\phi$ mais concentrada em torno de $\hat{\phi}$. A razào deste fato é que a quantidade $\sigma_{e g}^{2}$ aparece na matriz de variâncias e covariâncias de $\boldsymbol{\phi}$ levando a distribuição condicional $p\left(\phi \mid \sigma_{e g}^{2}, \boldsymbol{y}\right)$ a estar sujeita a duas restriçōes: $\sigma_{e g b}^{2}>\sigma_{e g}^{2}$ e $\sigma_{e g}^{2}>\sigma_{e}^{2}$. Enquanto a primeira restrição tende a reduzir a dispersão da distribuição de $\phi$ a última tende a incrementá-la. O efeito combinado dessas duas restriçōes depende das duas razões dos quadrados médios: $\frac{m_{e}}{m_{g}}$ e $\frac{m_{b}}{m_{g}}$. Em geral, quanto maior as razões $\frac{m_{e}}{m_{g}}$ e $\frac{m_{b}}{m_{g}}$, maior será a redução na dispersão da distribuição de $\phi$.

\subsubsection{Aproximando a Distribuição $p(\phi \mid y)$}

Embora seja possível se fazer inferências sobre $\phi$ empregando diretamente a distribuição a posteriori $p(\boldsymbol{\phi} \mid \boldsymbol{y})$ em (95), Box \& TiaO(1973) propõem que seja usada uma aproximação para $p(\boldsymbol{\phi} \mid \boldsymbol{y})$. Tal aproximação, deve-se ao fato de não ser possível expressar $p(\phi \mid \boldsymbol{y})$ de modo simples.

Para um dado conjunto de observaçōes o efeito específico da restrição $C: \sigma_{e g b}^{2}>\sigma_{e g}^{2}>\sigma_{e}^{2}$ pode ser aproximadamente determinado conforme procedimento a seguir.

A distribuição a posteriori de $\phi$, pode ser obtida por,

$$
p(\phi \mid y)=\int_{0}^{\infty} p\left(\phi \mid \sigma_{e g}^{2}, y\right) p\left(\sigma_{e g}^{2} \mid y\right) d \sigma_{e g}^{2}, \quad-\infty<\phi_{i}<+\infty, \quad i=1, \ldots, K-1
$$

onde

$$
p\left(\boldsymbol{\phi} \mid \sigma_{e g}^{2}, \boldsymbol{y}\right)=\sqrt{K}\left(\frac{J R}{2 \pi}\right)^{\frac{\nu_{\phi}}{2}}\left(\sigma_{e g}^{2}\right)^{-\frac{\nu_{e}}{2}} \exp \left\{-\frac{S(\boldsymbol{\phi})}{2 \sigma_{e g}^{2}}\right\} I_{(-\infty,+\infty)}(\boldsymbol{\phi}), \quad \sigma_{e g}^{2}>0
$$




$$
p\left(\sigma_{e g}^{2} \mid \boldsymbol{y}\right)=\int_{0}^{\sigma_{\varepsilon g}^{2}} \int_{\sigma_{e g}^{2}}^{\infty} p\left(\sigma_{e}^{2}, \sigma_{e g}^{2}, \sigma_{e g b}^{2} \mid \boldsymbol{y}\right) d \sigma_{e g b}^{2} d \sigma_{e}^{2}, \quad \sigma_{e g}^{2}>0 \quad e
$$

$p\left(\sigma_{\varepsilon}^{2}, \sigma_{e g}^{2}, \sigma_{e g b}^{2} \mid \boldsymbol{y}\right)$ já definido em (89).

\section{A proximando $p\left(\sigma_{e g}^{2} \mid \boldsymbol{y}\right)$ em Dois Estágios}

O primeiro estágio consiste em tornar $p\left(\sigma_{e}^{2}, \sigma_{e g}^{2} \mid \boldsymbol{y}\right)$ proporcional ao produto de duas $\chi^{2}$ 's simples. Para este fim, integra-se $p\left(\sigma_{e}^{2}, \sigma_{\text {eg }}^{2}, \sigma_{\text {egb }}^{2} \mid \boldsymbol{y}\right)$ em relação a $\sigma_{\text {egb }}^{2}$, isto é,

$$
\begin{aligned}
p\left(\sigma_{e}^{2}, \sigma_{e g}^{2} \mid \boldsymbol{y}\right)= & \frac{\left(\nu_{e} m_{e}\right)^{-1} p\left(\chi_{\nu_{e}}^{-2}=\frac{\sigma_{e}^{2}}{\nu_{e} m_{e}}\right)\left(\nu_{g} m_{g}\right)^{-1} p\left(\chi_{\nu_{g}}^{-2}=\frac{\sigma_{e g}^{2}}{\nu_{g} m_{g}}\right)}{\operatorname{Pr}\left\{\frac{\chi_{\nu_{e}}^{2}}{\chi_{\nu_{g}}^{2}}>\frac{\nu_{e} m_{e}}{\nu_{g} m_{g}} ; \frac{\chi_{\nu_{g}}^{2}}{\chi_{\nu_{b}}^{2}}>\frac{\nu_{g} m_{g}}{\nu_{b} m_{b}}\right\}} \times \\
& \frac{\left(\nu_{b} m_{b}\right)^{-1}}{\Gamma\left(\frac{\nu_{b}}{2}\right) 2^{\frac{\nu_{b}}{2}}} \int_{\sigma_{e g}^{2}}^{\infty}\left(\frac{\sigma_{e g b}^{2}}{\nu_{b} m_{b}}\right)^{-\left(\frac{\nu_{b}}{2}+1\right)} \exp \left\{-\frac{\nu_{b} m_{b}}{2 \sigma_{e g b}^{2}}\right\} d \sigma_{e g b}^{2} .
\end{aligned}
$$

Com a substituição $\sigma_{e g b}^{2}=\left(\nu_{b} m_{b}\right) U_{1}$, chega-se ao seguinte resultado

$$
\begin{aligned}
p\left(\sigma_{e}^{2}, \sigma_{e g}^{2} \mid \boldsymbol{y}\right)= & \delta\left(\nu_{e} m_{e}\right)^{-1} p\left(\chi_{\nu_{e}}^{2}=\frac{\sigma_{e}^{2}}{\nu_{e} m_{e}}\right)\left(\nu_{g} m_{g}\right)^{-1} p\left(\chi_{\nu_{g}}^{-2}=\frac{\sigma_{e g}^{2}}{\nu_{g} m_{g}}\right) \times \\
& \operatorname{Pr}\left\{\chi_{\nu_{b}}^{-2}>\frac{\sigma_{e g}^{2}}{\nu_{b} m_{b}}\right\}, \quad \sigma_{e g}^{2}>\sigma_{e}^{2}>0
\end{aligned}
$$

ou ainda,

$$
p\left(\sigma_{e}^{2}, \sigma_{e g}^{2} \mid y\right)=\delta^{\prime}\left(\nu_{e} m_{e}\right)^{-1} p\left(\chi_{\nu_{e}}^{2}=\frac{\sigma_{e}^{2}}{\nu_{e} m_{e}}\right) G_{1}\left(\sigma_{e g}^{2}\right), \quad \sigma_{e g}^{2}>\sigma_{e}^{2}>0,
$$

onde

$$
\begin{gathered}
\delta^{\prime}=\delta \operatorname{Pr}\left\{F_{\nu_{b}, \nu_{g}}<\frac{m_{b}}{m_{g}}\right\}, \\
G_{1}\left(\sigma_{e g}^{2}\right)=\frac{\left(\nu_{g} m_{g}\right)^{-1} p\left(\chi_{\nu_{g}}^{2}=\frac{\sigma_{g g}^{2}}{\nu_{g} m_{g}}\right) \operatorname{Pr}\left\{\chi_{\nu_{b}}^{2}<\frac{\nu_{b} m_{b}}{\sigma_{\varepsilon_{g}}^{2}}\right\}}{\operatorname{Pr}\left\{F_{\nu_{b}, \nu_{g}}<\frac{m_{b}}{m_{g}}\right\}}
\end{gathered}
$$

e

$$
\delta^{-1}=\operatorname{Pr}\left\{\frac{\chi_{\nu_{e}}^{2}}{\chi_{\nu_{g}}^{2}}>\frac{\nu_{e} m_{e}}{\nu_{g} m_{g}} ; \frac{\chi_{\nu_{g}}^{2}}{\chi_{\nu_{b}}^{2}}>\frac{\nu_{g} m_{g}}{\nu_{b} m_{b}}\right\}
$$


Agora, $G_{1}\left(\sigma_{e g}^{2}\right)$ pode ser escrita como,

$$
G_{1}\left(\sigma_{e g}^{2}\right)=\frac{\left(\nu_{g} m_{g}\right)^{-1} \operatorname{Pr}\left\{\chi_{\nu_{b}}^{2}<\frac{\nu_{b} m_{b}}{\sigma_{e g}^{2}}\right\}}{\operatorname{Pr}\left\{F_{\nu_{b}, \nu_{g}}<\frac{m_{b}}{m_{g}}\right\}} \frac{1}{\Gamma\left(\frac{\nu_{g}}{2}\right) 2^{\frac{\nu_{g}}{2}}}\left(\frac{\sigma_{e g}^{2}}{\nu_{g} m_{g}}\right)^{-\left(\frac{\nu_{g}}{2}+1\right)} e^{-\frac{\nu_{g} m_{g}}{2 \sigma_{e g}^{2}}} I_{(0, \infty)}\left(\sigma_{e g}^{2}\right) .
$$

Fazendo-se a mudança da variável para $Z=\frac{\nu_{g} m_{g}}{\sigma_{e g}^{2}}$, obtém-se

$$
p\left(Z=\frac{\nu_{g} m_{g}}{\sigma_{e g}^{2}} \mid \boldsymbol{y}\right)=\frac{\operatorname{Pr}\left\{\frac{\chi_{\nu_{b}}^{2}}{Z}<\frac{\nu_{b} m_{b}}{\nu_{g} m_{g}}\right\}}{\operatorname{Pr}\left\{F_{\nu_{b}, \nu_{g}}<\frac{m_{b}}{m_{g}}\right\} \Gamma\left(\frac{\nu_{g}}{2}\right) 2^{\frac{v_{g}}{2}}} z^{\frac{\nu_{g}}{2}-1} e^{-\frac{z}{2}} I_{(0, \infty)}(z)
$$

segue, portanto, que o $r$-ésimo momento da distribuição e a função geradora dos momentos de $Z$ serão dados, respectivamente, por

$$
E\left[Z^{r} \mid \boldsymbol{y}\right]=\int_{0}^{\infty} z^{r} p(z \mid \boldsymbol{y}) d z=2^{r} \frac{\Gamma\left(\frac{\nu_{g}+2 r}{2}\right)}{\Gamma\left(\frac{\nu_{g}}{2}\right)} \frac{\operatorname{Pr}\left\{F_{\nu_{b}, \nu_{g}+2 r}<\left(\frac{\nu_{g}+2 r}{\nu_{g}}\right) \frac{m_{b}}{m_{g}}\right\}}{\operatorname{Pr}\left\{F_{\nu_{b}, \nu_{g}}<\frac{m_{b}}{m_{g}}\right\}}
$$

$\mathrm{e}$

$$
M_{z}(t)=\int_{0}^{\infty} e^{t z} p(z \mid \boldsymbol{y}) d z=(1-2 t)^{-\frac{\nu_{g}}{2}} \frac{\operatorname{Pr}\left\{F_{\nu_{b}, \nu_{g}}<\left(\frac{1}{1-2 t}\right) \frac{m_{b}}{m_{g}}\right\}}{\operatorname{Pr}\left\{F_{\nu_{b}, \nu_{g}}<\frac{m_{b}}{m_{g}}\right\}}
$$

ou

$$
M_{z}(t)=(1-2 t)^{-\frac{\nu_{g}}{2}} \cdot \frac{\frac{\left(\frac{\nu_{b}}{\nu_{g}}\right)}{B\left(\frac{\nu_{g}}{2}\right)} \int_{0}^{\left(\frac{1}{2}\right)} \int_{0}^{\left(\frac{1}{1-2 t}\right) \frac{m_{b}}{m_{g}}}\left(\frac{\nu_{b}}{\nu_{g}} f\right)^{\frac{\nu_{b}}{2}-1}\left(1+\frac{\nu_{b}}{\nu_{g}} f\right)^{-\frac{\nu_{b}+\nu_{g}}{2}} d f}{\frac{\left(\frac{\nu_{g}}{\nu_{g}}\right)}{B\left(\frac{\nu_{g}}{2} \frac{\nu_{g}}{2}\right)} \int_{0}^{\left(\frac{m_{b}}{m_{g}}\right)}\left(\frac{\nu_{b}}{\nu_{g}} f\right)^{\frac{\nu_{b}}{2}-1}\left(1+\frac{\nu_{b}}{\nu_{g}} f\right)^{-\frac{\nu_{b}+\nu_{g}}{2}} d f} .
$$

Estudando $M_{z}(t)$ para valores extremos de $\frac{m_{b}}{m_{g}}$, tem-se que

(a) $\lim _{\frac{m_{b}}{m_{g}} \rightarrow \infty} M_{z}(t)=(1-2 t)^{-\frac{\nu_{g}}{2}}$, a qual é a função geradora dos momentos de uma variável aleatória com distribuição de $\chi_{\nu_{g}}^{2}$;

(b) Lembrando da regra de Leibnis para diferenciação de integrais, a saber,

$$
\frac{d}{d t}\left[\int_{g(t)}^{h(t)} f(x) d x\right]=f(h(t)) \frac{d h}{d t}-f(g(t)) \frac{d g}{d t}
$$

e empregando a regra de L'Hospital, que consiste em diferenciar o numerador e o denominador individualmente e em seguida aplicar o limite novamente, verificou-se que $\lim _{\frac{m_{b}}{m_{g}} \rightarrow 0} M_{z}(t)=(1-2 t)^{-\frac{\nu_{g}+\nu_{b}}{2}}$, a qual é a função geradora dos momentos de uma variável aleatória com distribuição $\chi_{\nu_{g}+\nu_{b}}^{2}$. 
Portanto, vê-se que para valores grandes de $\frac{m_{b}}{m_{g}}$ a variável $Z$ se aproxima de uma $\chi_{\nu_{g}}^{2}$ e quando $\frac{m_{b}}{m_{g}}$ se aproxima de zero, a variável aleatória $Z$ se aproxima de uma $\chi_{\nu_{g}+\nu_{b}}^{2}$. Este fato, juntamente com a forma da densidade de $Z$ dada em (104), sugere que a variável $Z$ seja aproximada por uma $\chi^{2}$ simples, isto é. $Z \dot{\sim} a \chi_{b}^{2}$, onde $a$ e $b$ serão obtidos equacionando os dois primeiros momentos da variável $Z$ com os dois primeiros momentos da variável $a \chi_{b}^{2}$.

Retomando a expressão (105), verifica-se que os dois primeiros momentos da variável aleatória $Z$ são, respectivamente,

$$
E[Z \mid y]=\nu_{g} \frac{P_{1}}{P_{3}} \quad \epsilon \quad E\left[Z^{2} \mid y\right]=\nu_{g}\left(\nu_{g}+2\right) \frac{P_{2}}{P_{3}}
$$

onde

$$
\begin{gathered}
P_{1}=\operatorname{Pr}\left\{F_{\nu_{b}, \nu_{g}+2}<\left(\frac{\nu_{g}+2}{\nu_{g}}\right) \frac{m_{b}}{m_{g}}\right\}=I_{y}\left(\frac{\nu_{b}}{2}, \frac{\nu_{g}}{2}+1\right), \\
P_{2}=\operatorname{Pr}\left\{F_{\nu_{b}, \nu_{g}+4}<\left(\frac{\nu_{g}+4}{\nu_{g}}\right) \frac{m_{b}}{m_{g}}\right\}=I_{y}\left(\frac{\nu_{b}}{2}, \frac{\nu_{g}}{2}+2\right), \\
P_{3}=\operatorname{Pr}\left\{F_{\nu_{b}, \nu_{g}}<\frac{m_{b}}{m_{g}}\right\}=I_{y}\left(\frac{\nu_{b}}{2}, \frac{\nu_{g}}{2}\right) ; \\
y=\frac{\nu_{b} m_{b}}{\nu_{b} m_{b}+\nu_{g} m_{g}} \text { e } I_{y}(p, q)=\frac{1}{B(p, q)} \int_{0}^{y} u^{p-1}(1-u)^{q-1} d u .
\end{gathered}
$$

Por outro lado tem-se que

$$
E\left[a \chi_{b}^{2}\right]=a b \quad e \quad E\left[\left(a \chi_{b}^{2}\right)^{2}\right]=a^{2} b^{2}+2 a^{2} b
$$

Daí.

$$
a b \doteq \nu_{g} \frac{P_{1}}{P_{3}} \Longrightarrow b=\frac{\nu_{g}}{a} \frac{P_{1}}{P_{3}} \quad e \quad a=\frac{\left(\nu_{g}+2\right)}{2} \frac{P_{2}}{P_{1}}-\frac{\nu_{g}}{2} \frac{P_{1}}{P_{3}}
$$

Agra,

$$
Z=\frac{\nu_{g} m_{g}}{\sigma_{e g}^{2}} \dot{\sim} a \chi_{b}^{2} \Longrightarrow \sigma_{e g}^{2} \dot{\sim} \frac{\nu_{g} m_{g}}{a} \chi_{b}^{-2} \Longrightarrow\left\{\begin{array}{l}
b=\nu_{g}^{\prime} \\
\frac{\nu_{g} m_{g}}{a}=\nu_{g}^{\prime} m_{g}^{\prime} \Longrightarrow m_{g}^{\prime}=\frac{\nu_{g} m_{g}}{a \nu_{g}^{\prime}}
\end{array}\right.
$$

Por esta aproximação, $G_{1}\left(\sigma_{e g}^{2}\right)$ pode ser escrita aproximadamente como

$$
G_{1}\left(\sigma_{e g}^{2}\right) \doteq\left(\nu_{g}^{\prime} m_{g}^{\prime}\right)^{-1} p\left(\chi_{\nu_{g}^{\prime}}^{-2}=\frac{\sigma_{e g}^{2}}{\nu_{g}^{\prime} m_{g}^{\prime}}\right), \quad \sigma_{e g}^{2}>0
$$


e consequentemente, a distribuição a posteriori de $\left(\sigma_{e}^{2}, \sigma_{e g}^{2}\right)$, dada em (102), pode ser escrita aproximadamente como

$$
p\left(\sigma_{e}^{2}, \sigma_{e g}^{2} \mid y\right) \doteq \delta^{\prime}\left(\nu_{e} m_{e}\right)^{-1} p\left(\chi_{\nu_{e}}^{-2}=\frac{\sigma_{e}^{2}}{\nu_{e} m_{e}}\right)\left(\nu_{g}^{\prime} m_{g}^{\prime}\right)^{-1} p\left(\chi_{\nu_{g}^{\prime}}^{-2}=\frac{\sigma_{e g}^{2}}{\nu_{g}^{\prime} m_{g}^{\prime}}\right), \quad \sigma_{e g}^{2}>\sigma_{e}^{2}>0
$$

O segundo estágio consiste em aproximar $p\left(\sigma_{\text {eg }}^{2} \mid \boldsymbol{y}\right)$ por uma simples $\chi^{2}$. Isto é, $\sigma_{e g}^{2} \dot{\sim} \nu_{g}^{*} m_{g}^{*} \chi_{\nu_{g}^{*}}^{-2}$. Neste sentido, integrando-se $p\left(\sigma_{e}^{2}, \sigma_{e g}^{2} \mid \boldsymbol{y}\right)$ dada em (107) em relação a $\sigma_{e}^{2}$, tem-se que

$$
p\left(\sigma_{e g}^{2} \mid y\right) \doteq \delta^{\prime}\left(\nu_{g}^{\prime} m_{g}^{\prime}\right)^{-1} p\left(\chi_{\nu_{g}^{\prime}}^{-2}=\frac{\sigma_{e g}^{2}}{\nu_{g}^{\prime} m_{g}^{\prime}}\right) \frac{\left(\nu_{e} m_{e}\right)^{-1}}{\Gamma\left(\frac{\nu_{e}}{2}\right) 2^{\frac{\nu_{e}}{2}}} \int_{0}^{\sigma_{\varepsilon g}^{2}}\left(\frac{\sigma_{e}^{2}}{\nu_{e} m_{e}}\right)^{-\left(\frac{\nu_{e}}{2}+1\right)} e^{-\frac{\nu_{e} m_{e}}{2 \sigma_{e}^{2}}} d \sigma_{e}^{2} .
$$

Com a substituição $\sigma_{e}^{2}=\left(\nu_{e} m_{e}\right) U_{2}$, a distribuição marginal a posteriori aproximada de $\sigma_{e g}^{2}$, resultante é

$$
p\left(\sigma_{e g}^{2} \mid \boldsymbol{y}\right) \doteq \delta^{\prime}\left(\nu_{g}^{\prime} m_{g}^{\prime}\right)^{-1} p\left(\chi_{\nu_{g}^{\prime}}^{-2}=\frac{\sigma_{e g}^{2}}{\nu_{g}^{\prime} m_{g}^{\prime}}\right) \operatorname{Pr}\left\{\chi_{\nu_{e}}^{-2}<\frac{\sigma_{e g}^{2}}{\nu_{e} m_{e}}\right\}, \quad \sigma_{e g}^{2}>0 .
$$

Mas esta distribuição pode ser escrita com

$$
p\left(\sigma_{e g}^{2} \mid \boldsymbol{y}\right) \doteq \frac{\delta^{\prime \prime}\left(\nu_{g}^{\prime} m_{g}^{\prime}\right)^{-1} p\left(\chi_{\nu_{g}^{\prime}}^{-2}=\frac{\sigma_{e g}^{2}}{\nu_{g}^{\prime} m_{g}^{\prime}}\right) \operatorname{Pr}\left\{\chi_{\nu_{e}}^{2}>\frac{\nu_{e} m_{e}}{\sigma_{e g}^{2}}\right\}}{\operatorname{Pr}\left\{F_{\nu_{e}, \nu_{g}^{\prime}}>\frac{m_{e}}{m_{g}^{\prime}}\right\}}, \quad \sigma_{e g}^{2}>0
$$

onde

$$
\delta^{\prime \prime}=\delta^{\prime} \operatorname{Pr}\left\{F_{\nu_{\varepsilon}, \nu_{g}^{\prime}}>\frac{m_{e}}{m_{g}^{\prime}}\right\} .
$$

Observa-se que a distribuição aproximada de $P\left(\sigma_{e g}^{2} \mid \boldsymbol{y}\right)$ dada em (112) não fica expressa em termos de uma função simples, pois para confeccionar seu gráfico, é preciso que se calcule duas probabilidades para estabelecer cada ponto da distribuição. Este fato sugere que a distribuição seja aproximada por uma $\chi^{2}$ simples, cujo procedimento é similar a aquele usado anteriormente para aproximar a distribuição a posteriori de $\left(\sigma_{e}^{2}, \sigma_{e g}^{2}\right)$.

Se for ignorada a constante $\delta^{\prime \prime}$, a distribuição $p\left(\sigma_{e g}^{2} \mid \boldsymbol{y}\right)$ dada em (112) fica semelhante a $G_{1}\left(\sigma_{e g}\right)$ dada em (103), de modo que se for feita a mudança 
da variável $\sigma_{\epsilon g}^{2}$ para $Z=\frac{\left(\nu_{g}^{\prime} m_{g}^{\prime}\right)}{\sigma_{e g}^{2}}$, a distribuição aproximada resultante de $Z$ será dada por

$$
p(z \mid \boldsymbol{y}) \doteq \frac{\operatorname{Pr}\left\{\frac{Z}{\chi_{\nu_{e}}^{2}}<\frac{\nu_{g}^{\prime} m_{g}^{\prime}}{\nu_{e} m_{e}}\right\} \quad 1}{\operatorname{Pr}\left\{F_{\nu_{g}^{\prime}, \nu_{e}}<\frac{m_{g}^{\prime}}{m_{e}}\right\} \Gamma\left(\frac{\nu_{g}^{\prime}}{2}\right) 2^{\frac{\nu_{g}^{\prime}}{2}}} z^{\frac{\nu_{g}^{\prime}}{2}-1} e^{-\frac{z}{2}} I_{(0, \infty)}(z)
$$

cujo r-ésimo momento de $Z$ será dado por

$$
E\left[Z^{r} \mid \boldsymbol{y}\right]=2^{r} \frac{\Gamma\left(\frac{\nu_{g}^{\prime}+2 r}{2}\right)}{\Gamma\left(\frac{\nu_{g}^{\prime}}{2}\right)} \frac{\operatorname{Pr}\left\{F_{\nu_{g}^{\prime}+2 r, \nu_{e}}<\left(\frac{\nu_{g}^{\prime}}{\nu_{g}^{\prime}+2 r}\right) \frac{m_{g}^{\prime}}{m_{e}}\right\}}{\operatorname{Pr}\left\{F_{\nu_{g}^{\prime}, \nu_{t}}<\frac{m_{g}^{\prime}}{m_{e}}\right\}}
$$

Igualando os dois primeiros momentos da distribuição de $Z$ aos dois primeiros momentos da distribuição da variável $c \chi_{d}^{2}$, vem

$$
E[Z \mid y]=\nu_{g}^{\prime} \frac{P_{4}}{P_{6}} \doteq c d \Longrightarrow d=\frac{\nu_{g}^{\prime}}{c} \frac{P_{4}}{P_{6}}
$$

e

$$
E\left[Z^{2} \mid y\right]=\nu_{g}^{\prime}\left(\nu_{g}^{\prime}+2\right) \frac{P_{5}}{P_{6}} \Longrightarrow c=\frac{\left(\nu_{g}^{\prime}+2\right)}{2} \frac{P_{5}}{P_{4}}-\frac{\nu_{g}^{\prime}}{2} \frac{P_{4}}{P_{6}}
$$

onde

$$
\begin{gathered}
P_{4}=\operatorname{Pr}\left\{F_{\nu_{g}^{\prime}+2, \nu_{e}}<\left(\frac{\nu_{g}^{\prime}}{\nu_{g}^{\prime}+2}\right) \frac{m_{g}^{\prime}}{m_{e}}\right\}=I_{x}\left(\frac{\nu_{g}^{\prime}}{2}+1, \frac{\nu_{e}}{2}\right), \\
P_{5}=\operatorname{Pr}\left\{F_{\nu_{g}^{\prime}+4, \nu_{e}}<\left(\frac{\nu_{g}^{\prime}}{\nu_{g}^{\prime}+4}\right) \frac{m_{g}^{\prime}}{m_{e}}\right\}=I_{x}\left(\frac{\nu_{g}^{\prime}}{2}+2, \frac{\nu_{e}}{2}\right), \\
P_{6}=\operatorname{Pr}\left\{F_{\nu_{g}^{\prime}, \nu_{e}}<\frac{m_{g}^{\prime}}{m_{e}}\right\}=I_{x}\left(\frac{\nu_{g}^{\prime}}{2}, \frac{\nu_{e}}{2}\right), \\
x=\frac{\nu_{g}^{\prime} m_{g}^{\prime}}{\nu_{g}^{\prime} m_{g}^{\prime}+\nu_{e} m_{e}} \text { e } I_{x}(p, q)=\frac{1}{B(p, q)} \int_{0}^{\infty} u^{p-1}(1-u)^{q-1} d u
\end{gathered}
$$

Agora

$$
Z=\frac{\nu_{g}^{\prime} m_{g}^{\prime}}{\sigma_{e g}^{2}} \dot{\sim} c \chi_{d}^{2} \Longrightarrow \sigma_{e g}^{2} \dot{\sim} \frac{\nu_{g}^{\prime} m_{g}^{\prime}}{c} \chi_{d}^{2} \Longrightarrow\left\{\begin{array}{l}
d=\nu_{g}^{*} \\
\frac{\nu_{g}^{\prime} m_{g}^{\prime}}{c}=\nu_{g}^{*} m_{g}^{*} \Longrightarrow m_{g}^{*}=\frac{\nu_{g}^{\prime} m_{g}^{\prime}}{c \nu_{g}^{*}}
\end{array}\right.
$$

Para este grau de aproximação, a distribuição marginal a posteriori de $\sigma_{\varepsilon g}^{2}$ será dada, aproximadamente, por

$$
p\left(\sigma_{e g}^{2} \mid \boldsymbol{y}\right) \doteq\left(\nu_{g}^{*} m_{g}^{*}\right)^{-1} p\left(\chi_{\nu_{3}^{*}}^{-2}=\frac{\sigma_{e g}^{2}}{\nu_{g}^{*} m_{g}^{*}}\right) I_{(0, \infty)}\left(\sigma_{e g}^{2}\right) .
$$


Agora, substituindo-se este resultado em (97), tem-se uma aproximação para a distribuição a posteriori de $\phi$, isto é,

$$
\begin{aligned}
& p(\boldsymbol{\phi} \mid \boldsymbol{y}) \doteq \int_{0}^{\infty} p\left(\boldsymbol{\phi} \mid \sigma_{e g}^{2}, \boldsymbol{y}\right)\left(\nu_{g}^{*} m_{g}^{*}\right)^{-1} p\left(\chi_{\nu_{g}^{*}}^{-2}=\frac{\sigma_{e g}^{2}}{\nu_{g}^{*} m_{g}^{*}}\right) d \sigma_{e g}^{2} \\
& \doteq \sqrt{K}\left(\frac{J R}{2 \pi}\right)^{\frac{\nu_{\boldsymbol{\phi}}}{2}} \int_{0}^{\infty}\left(\sigma_{e g}^{2}\right)^{-\frac{\nu_{\phi}}{2}} e^{-\frac{s(\boldsymbol{\phi})}{2 \sigma_{e g}^{2}}} \frac{\left(\nu_{g}^{*} m_{g}^{*}\right)^{-1}}{\Gamma\left(\frac{\nu_{g}^{*}}{2}\right) 2^{\frac{\nu_{g}^{*}}{2}}}\left(\frac{\sigma_{e g}^{2}}{\nu_{g}^{*} m_{g}^{*}}\right)^{-\left(\frac{\nu_{g}^{*}}{2}+1\right)} e^{-\frac{\nu_{g}^{*} m_{g}^{*}}{2 \sigma_{g g}^{2}}} d \sigma_{e g}^{2}
\end{aligned}
$$

que após algumas operações algébricas, chega-se a

$$
\begin{aligned}
p(\phi \mid y) \doteq & \frac{\left|\sum\right|^{-\frac{1}{2}}\left(m_{g}^{*}\right)^{-\frac{\nu_{\phi}}{2}} \Gamma\left(\frac{\nu_{g}^{*}+\nu_{\phi}}{2}\right)}{\left[\Gamma\left(\frac{1}{2}\right)\right]^{\nu_{\phi}} \Gamma\left(\frac{\nu_{g}^{*}}{2}\right)\left(\nu_{g}^{*}\right)^{\frac{\nu_{g}^{*}}{2}}}\left[1+\frac{\left.(\phi-\hat{\phi})^{\prime} \sum^{-1}(\phi-\hat{\phi})\right]^{-\frac{\nu_{g}^{*}+\nu_{\phi}}{2}}}{\nu_{g}^{*} m_{g}^{*}},\right. \\
& -\infty<\phi_{i}<+\infty, \quad i=1,2, \ldots, K-1,
\end{aligned}
$$

a qual é a densidade de uma t-Student multivariada $(K-1)$-dimensional, com vetor de médias $\hat{\phi}$, matriz de variâncias e covariâncias $m_{g}^{*} \sum$ e $\nu_{g}^{*}$ graus de liberdade, denotada por $t_{(K-1)}\left(\hat{\phi}, m_{g}^{*} \sum, \nu_{g}^{*}\right)$. Sendo assim, o efeito aproximado do fator modificador $g(\phi)$ é apenas a mudança na escala do fator $\left(m_{g}\right)^{\frac{1}{2}}$ para $\left(m_{g}^{*}\right)^{\frac{1}{2}}$ e a mudança nos graus de liberdade de $\nu_{g}$ para $\nu_{g}^{*}$.

\subsubsection{Inferências Conjuntas e Marginais Sobre $\phi$ na Análise do Grupo de $J$ Experimentos Completamente Aleatorizados}

Geralmente há interesse em se fazer comparações gerais de todos os $K$ efeitos médios dos tratamentos $\boldsymbol{\theta}=\left(\theta_{1}, \theta_{2}, \ldots, \theta_{K}\right)^{\prime}$. Para tal finalidade, deve ser tomado qualquer conjunto de $(K-1)$ contrastes não redundantes $\phi=$ $\left(\phi_{1}, \phi_{2}, \ldots, \phi_{K-1}\right)^{\prime}$ e determinar a região H.P.D. de conteúdo $(1-\alpha)$, aproximada, com base no fato de que

$$
\frac{S(\phi)}{\nu_{\phi} m_{g}^{*}}=\frac{m(\phi)}{m_{g}^{*}} \dot{\sim} F_{\left[\nu_{\phi}, \nu_{g}^{*}, \alpha\right]} .
$$

Particularmente, verificar a hipótese $H_{0}: \theta_{1}=\ldots=\theta_{K}=\theta$ (para algum $\theta$ desconhecido) a um nível de significância $\alpha$, é equivalente a estudar a distribuição conjunta a posteriori de $\phi=\left(\phi_{1}, \ldots, \phi_{K-1}\right)^{\prime}$ e verificar se o ponto $\phi_{0}=$ $\left(\phi_{10}=0, \phi_{20}=0, \ldots, \phi_{(K-1) 0}=0\right)$ ' está dentro ou fora da região H.P.D. de conteúdo 
$(1-\alpha)$. Portanto, o ponto $\phi_{0}=\mathbf{0}\left(\phi_{10}=\theta_{1}-\bar{\theta}=0, \phi_{20}=\theta_{2}-\bar{\theta}=0, \ldots, \phi_{(K-1) 0}=\right.$ $\left.\boldsymbol{\theta}_{\left(K^{\prime}-1\right)}-\bar{\theta}=0\right)$ ' estará incluído na região H.P.D. de conteúdo $(1-\alpha)$ se, e somente se,

$$
\frac{S\left(\phi_{0}\right)}{\nu_{\phi} m_{g}^{*}}=\frac{\left(\phi_{0}-\hat{\phi}\right)^{\prime} \sum^{-1}\left(\phi_{0}-\hat{\phi}\right)}{\nu_{\phi} m_{g}^{*}}=\frac{J R \sum_{i=1}^{K}\left(\bar{y}_{i,}-\bar{y}_{\ldots}\right)^{2}}{\nu_{\phi} m_{g}^{*}}<F_{\left[\nu_{\phi}, \nu_{g}^{*}, \alpha\right]} .
$$

Se for de interesse comparar os efeitos médios de dois tratamentos, isto pode ser feito, usando o fato de que $\delta=\theta_{i}-\theta_{s}, i \neq s,(i, s=1,2, \ldots, K)$, é aproximadamente distribuído como uma t-Student univariada de média $\hat{\delta}=\bar{y}_{i . .}-\bar{y}_{s .}$, variância $\frac{2}{J R} m_{g}^{*}$ e $\nu_{g}^{*}$ graus de liberdade, denotada por, $t_{1}\left(\hat{\delta}, \frac{2}{J R} m_{g}^{*}, \nu_{g}^{*}\right)$. Para fazer isto, basta construir o intervalo de credibilidade a posteriori, com conteúdo $(1-\alpha)$, aproximado, para $\delta$, ou seja,

$$
\operatorname{Pr}\left\{\hat{\delta}-t_{\frac{\alpha}{2}} \sqrt{\frac{2}{J R} m_{g}^{*}} \leq \delta \leq \hat{\delta}+t_{\frac{\alpha}{2}} \sqrt{\frac{2}{J R} m_{g}^{*}}\right\} \doteq 1-\alpha
$$

e verificar se o ponto zero está dentro ou fora deste intervalo.

Dentre os grupos de $(K-1)$ contrastes não redundantes $\phi$ dos efeitos médios dos tratamentos $\boldsymbol{\theta}$, (ou seja $\boldsymbol{\phi}=D \boldsymbol{\theta}$ ) os contrastes ortogonais são de especial interesse neste trabalho. Sendo assim, a quantidade $S(\phi)$ associada a problemas desta natureza é dada por

$$
S(\phi)=(\phi-\hat{\phi})^{\prime}\left[D\left(X^{\prime} X\right)^{-1} D^{\prime}\right]^{-1}(\phi-\hat{\phi})
$$

onde $\left(X^{\prime} X\right)^{-1}=\operatorname{Diag}\left\{\frac{1}{J R}, \ldots, \frac{1}{J R}\right\}$ com dimensão $(K-1), D=\left(d_{j i}\right), i=1, \ldots, K$, $j=1, \ldots,(K-1), \sum_{i=1}^{K} d_{j i}=0, \sum_{i=1}^{K} d_{j i} d_{j^{\prime} i}=0, j \neq j^{\prime}$ e $\hat{\phi}_{j}=\sum_{i=1}^{K} d_{j i} \bar{y}_{i . .}$ Nestas condiçōes, o ponto $\phi_{0}=\left(\phi_{10}=0, \phi_{20}=0, \ldots, \phi_{(K-1) 0}=0\right)^{\prime}$ ' estará incluído na região H.P.D. de conteúdo $(1-\alpha)$ se, e somente se,

$S\left(\phi_{0}=0\right)=\frac{\left(\sum_{i=1}^{K} d_{1 i} \bar{y}_{i . .}\right)^{2}}{\sum_{i=1}^{K} \frac{d_{1 i}^{2}}{J R}}+\frac{\left(\sum_{i=1}^{K} d_{2 i} \bar{y}_{i . .}\right)^{2}}{\sum_{i=1}^{K} \frac{d_{2 i}^{2}}{J R}}+\ldots+\frac{\left(\sum_{i=1}^{K} d_{(K-1) i} \bar{y}_{i . .}\right)^{2}}{\sum_{i=1}^{K} \frac{d_{(K-1) i}^{2}}{J R}}<\nu_{\phi} m_{g}^{*} F_{\left[\nu_{\phi}, \nu_{g}^{*}, \alpha\right]}$. 
Ademais, inferências marginais aproximadas acerca de um subconjunto de $q$ contraste ortogonais $\phi^{q}=\left(\phi_{1}, \phi_{2}, \ldots, \phi_{q}\right)^{\prime}, q<(K-1)$, podem ser feitas, com base no fato de que

$$
\frac{S\left(\phi^{q}\right)}{q m_{g}^{*}}=\frac{\left(\phi^{q}-\hat{\phi}^{q}\right)^{\prime}\left[D_{q}\left(X_{q}^{\prime} X_{q}\right)^{-1} D_{q}^{\prime}\right]^{-1}\left(\phi^{q}-\hat{\phi}^{q}\right)}{q m_{g}^{*}} \dot{\sim} F_{\left[q, \nu_{g}^{*}, \alpha\right]}
$$

onde, $\hat{\phi}^{q}=\left(\hat{\phi}_{1}, \hat{\phi}_{2}, \ldots, \hat{\phi}_{q}\right),\left(X_{q}^{\prime} X_{q}\right)^{-1}=\operatorname{Diag}\left\{\frac{1}{J R}, \ldots, \frac{1}{J R}\right\}$ de posto $q$ e $D_{q}=\left(d_{j i}\right)$, $i=1,2, \ldots, K, j=1,2, \ldots, q$ é a matriz dos coeficientes dos $q$ contrastes ortogonais.

Verificar a hipótese $H_{0}: \phi^{q}=0$ a um nível de significância $\alpha$ é equivalente a tomar a distribuição conjunta a posteriori de $\boldsymbol{\phi}^{q}=\left(\phi_{1}, \phi_{2}, \ldots, \phi_{q}\right)^{\prime} \mathrm{e}$ verificar se o ponto particular $\phi_{0}^{q}=\left(\phi_{10}=0, \phi_{20}=0, \ldots, \phi_{q 0}=0\right)$ ' está incluído ou excluído da região H.P.D. de conteúdo $(1-\alpha)$. Neste sentido, o ponto $\phi_{0}^{q}=\mathbf{0}$ estará incluído na região H.P.D. de conteúdo $(1-\alpha)$ se, e somente se,

$$
S\left(\phi_{0}^{q}=0\right)=\frac{\left(\sum_{i=1}^{K} d_{1 i} \bar{y}_{i . .}\right)^{2}}{\sum_{i=1}^{K} \frac{d_{1 i}^{2}}{J R}}+\frac{\left(\sum_{i=1}^{K} d_{2 i} \bar{y}_{i . .}\right)^{2}}{\sum_{i=1}^{K} \frac{d_{2 i}^{2}}{J R}}+\ldots+\frac{\left(\sum_{i=1}^{K} d_{q i} \bar{y}_{i . .}\right)^{2}}{\sum_{i=1}^{K} \frac{d_{q i}^{2}}{J R}}<q m_{g}^{*} F_{\left\{q, \nu_{g}^{*}, \alpha\right\}}
$$

Em particular, inferências marginais aproximadas sobre qualquer contraste $\phi_{j}$, podem ser feitas, usando o fato de que

$$
\phi_{j} \mid \boldsymbol{y} \dot{\sim} t_{1}\left[\hat{\phi}_{j}, \frac{m_{g}^{*}}{J R} \sum_{i=1}^{K} d_{j i}^{2}, \nu_{g}^{*}\right] .
$$

Verificar a hipótese $H_{0}: \phi_{j}=0$ a um nível de significância $\alpha$, é equivalente a construir o intervalo de credibilidade $a$ posteriori com conteúdo $(1-\alpha)$, dado por

$$
\operatorname{Pr}\left\{\hat{\phi}_{j}-t_{\frac{\alpha}{2}} \sqrt{\frac{m_{g}^{*}}{J R} \sum_{i=1}^{K} d_{j i}^{2}} \leq \phi_{j} \leq \hat{\phi}_{j}+t_{\frac{\alpha}{2}} \sqrt{\frac{m_{g}^{*}}{J R} \sum_{i=1}^{K} d_{j i}^{2}}\right\} \doteq 1-\alpha
$$

e verificar se o ponto zero está dentro ou fora deste intervalo.

\subsubsection{Distribuição Marginal a Posteriori de $\sigma_{e}^{2}$}

Em geral, na prática, interessa ao pesquisador o estudo do comportamento probabilístico dos componentes da variância, marginalmente ou de relações 
entre eles. No presente estudo, trabalhou-se apenas com as distribuições marginais a posteriori de tais componentes.

Para determinar a distribuição posteriori de $\sigma_{e}^{2}$, é preciso integrar $p\left(\sigma_{e}^{2}, \sigma_{e g}^{2}, \sigma_{e g b}^{2} \mid \boldsymbol{y}\right)$, dada em (89), em relação a $\sigma_{e g}^{2}$ e $\sigma_{e g b}^{2}$, ou seja,

$$
\begin{aligned}
p\left(\sigma_{e}^{2} \mid \boldsymbol{y}\right)=\int_{\sigma_{s}}^{\infty} \int_{\sigma_{e g}^{2}}^{\infty} p\left(\sigma_{e}^{2}, \sigma_{e g}^{2}, \sigma_{e g b}^{2} \mid \boldsymbol{y}\right) d \sigma_{e g b}^{2} d \sigma_{e g}^{2} \\
=\delta\left(\nu_{e} m_{e}\right)^{-1} p\left(\chi_{\nu_{e}}^{-2}=\frac{\sigma_{e}^{2}}{\nu_{e} m_{e}}\right) \frac{\left(\nu_{g} m_{g}\right)^{-1}}{\Gamma\left(\frac{\nu_{g}}{2}\right) 2^{\frac{\nu_{g}}{2}}} \int_{\sigma_{e}^{2}}^{\infty}\left(\frac{\sigma_{e g}^{2}}{\nu_{g} m_{g}}\right)^{-\left(\frac{\nu_{g}}{2}+1\right)} \cdot \exp \left\{-\frac{\nu_{g} m_{g}}{2 \sigma_{e g}^{2}}\right\} \\
\quad \times \frac{\left(\nu_{b} m_{b}\right)^{-1}}{\Gamma\left(\frac{\nu_{b}}{2}\right) 2^{\frac{\nu_{b}}{2}}} \int_{\sigma_{e g}^{2}}^{\infty}\left(\frac{\sigma_{e g b}^{2}}{\nu_{b} m_{b}}\right)^{-\left(\frac{\nu_{b}}{2}+1\right)} \exp \left\{-\frac{\nu_{b} m_{b}}{2 \sigma_{e g b}^{2}}\right\} d \sigma_{e g b}^{2} d \sigma_{e g}^{2},
\end{aligned}
$$

a qual, após algumas operações algébricas, resulta em

$$
\begin{aligned}
p\left(\sigma_{e}^{2} \mid \boldsymbol{y}\right)= & \delta\left(\nu_{e} m_{e}\right)^{-1} p\left(\chi_{\nu_{e}}^{-2}=\frac{\sigma_{e}^{2}}{\nu_{e} m_{e}}\right) \\
& \times \int_{\sigma_{e}^{2}}^{\infty}\left(\nu_{g} m_{g}\right)^{-1} p\left(\chi_{\nu_{g}}^{-2}=\frac{\sigma_{e g}^{2}}{\nu_{g} m_{g}}\right) \operatorname{Pr}\left\{\chi_{\nu_{b}}^{2}<\frac{\nu_{b} m_{b}}{\sigma_{e g}^{2}}\right\} d \sigma_{e g}^{2} .
\end{aligned}
$$

Em palavras, a distribuição posteriori de $\sigma_{e}^{2}$ é proporcional ao produto de dois fatores: o primeiro, $\left(\nu_{e} m_{e}\right)^{-1} p\left(\chi_{\nu_{e}}^{-2}=\frac{\sigma_{e}^{2}}{\nu_{e} m_{e}}\right)$, é a densidade de uma qui-quadrado inversa com $\nu_{e}$ graus de liberdade, a qual representa a distribuição posteriori de $\sigma_{e}^{2}$, se a restrição $C: \sigma_{e g b}^{2}>\sigma_{e g}^{2}>\sigma_{e}^{2}$ for ignorada. E, o segundo fator, uma integral dupla, é induzido pela restrição $C$. Devido a esta integral ser uma quantidade monotonicamente decrescente de $\sigma_{e}^{2}$, o efeito da restrição desloca a distribuição a posteriori de $\sigma_{e}^{2}$ para esquerda de uma $\chi^{-2}$. Avaliações exatas da distribuição posteriori de $\sigma_{e}^{2}$ são bastante trabalhosas até com auxilio de computadores. Contudo, para avaliações aproximadas, pode-se usar a distribuição posteriori aproximada por uma simples qui-quadrado. A técnica consiste em igualar os dois primeiros momentos da distribuição da variável $Z=\frac{\nu_{e} m_{e}}{\sigma_{e}^{2}}$ aos dois primeiros momentos de uma $e \chi_{f}^{2}$, semelhante ao que foi feito na seção anterior. Tomando-se a expressão em (131) e fazendo-se as substituições $\sigma_{e g b}^{2}=\left(\nu_{b} m_{b}\right) U_{1}$ e $\sigma_{e g}^{2}=\left(\nu_{g} m_{g}\right) U_{2}$ 
na segunda e primeira integrais, respectivamente, chega-se ao seguinte resultado,

$$
\begin{aligned}
p\left(\sigma_{e}^{2} \mid y\right)= & \frac{\operatorname{Pr}\left\{\chi_{\nu_{g}}^{-2}>\frac{\sigma_{s}^{2}}{\nu_{g} m_{g}} ; \frac{\chi_{\nu_{g}}^{2}}{\chi_{\nu_{b}}^{2}}>\frac{\nu_{g} m_{g}}{\nu_{b} m_{b}}\right\}}{\operatorname{Pr}\left\{\frac{\chi_{\nu_{e}}^{2}}{\chi_{\nu_{g}}^{2}}>\frac{\nu_{e} m_{s}}{\nu_{g} m_{g}} ; \frac{\chi_{\nu_{g}}^{2}}{\chi_{\nu_{b}}^{2}}>\frac{\nu_{g} m_{g}}{\nu_{b} m_{b}}\right\}} \frac{\left(\nu_{e} m_{e}\right)^{-1}}{\Gamma\left(\frac{\nu_{e}}{2}\right) 2^{\frac{\nu_{s}}{2}}}\left(\frac{\sigma_{e}^{2}}{\nu_{e} m_{e}}\right)^{-\left(\frac{\nu_{s}}{2}+1\right)} e^{-\frac{\nu_{e} m_{e}}{2 \sigma_{\varepsilon}^{2}}} \\
& \sigma_{e}^{2}>0
\end{aligned}
$$

Usando a transformação $Z=\frac{\nu_{e} m_{e}}{\sigma_{e}^{2}}$, tem-se que a distribuição posteriori de $Z$ fica

$$
p(z \mid \boldsymbol{y})=\frac{\operatorname{Pr}\left\{\frac{Z}{\chi_{\nu_{g}}^{2}}>\frac{\nu_{e} m_{g}}{\nu_{g} m_{g}} ; \frac{\chi_{\nu_{g}}^{2}}{\chi_{\nu_{b}}^{2}}>\frac{\nu_{g} m_{g}}{\nu_{b} m_{b}}\right\}}{\operatorname{Pr}\left\{\frac{\chi_{\nu_{e}}^{2}}{\chi_{\nu_{g}}^{2}}>\frac{\nu_{e} m_{e}}{\nu_{g} m_{g}} ; \frac{\chi_{\nu_{g}}^{2}}{\chi_{\nu_{b}}^{2}}>\frac{\nu_{g} m_{g}}{\nu_{b} m_{b}}\right\}} \frac{1}{\Gamma\left(\frac{\nu_{g}}{2}\right) 2^{\frac{\nu_{g}}{2}}} z^{\left(\frac{\nu_{e}}{2}-1\right)} e^{-\frac{z}{2}} I_{(0, \infty)}(z) .
$$

Desde que $Z$ seja distribuida como uma $\chi_{\nu}^{2}$ o r-ésimo momento da distribuição será dado por

$$
E\left[Z^{r} \mid \boldsymbol{y}\right]=\int_{0}^{\infty} z^{r} p(z \mid \boldsymbol{y}) d z=2^{r} \frac{\Gamma\left(\frac{\nu_{e}+2 r}{2}\right)}{\Gamma\left(\frac{\nu_{g}}{2}\right)} \frac{\operatorname{Pr}\left\{\frac{\chi_{\nu_{e}+2 r}^{2}}{\chi_{\nu_{g}}^{2}}>\frac{\nu_{e} m_{e}}{\nu_{g} m_{g}} ; \frac{\chi_{\nu_{g}}^{2}}{\chi_{\nu_{b}}^{2}}>\frac{\nu_{g} m_{g}}{\nu_{b} m_{b}}\right\}}{\operatorname{Pr}\left\{\frac{\chi_{\nu_{g}}^{2}}{\chi_{\nu_{g}}^{2}}>\frac{\nu_{e} m_{e}}{\nu_{g} m_{g}} ; \frac{\chi_{\nu_{g}}^{2}}{\chi_{\nu_{b}}^{2}}>\frac{\nu_{g} m_{g}}{\nu_{b} m_{b}}\right\}}
$$

cujo primeiro e segundo momentos da distribuição resultará, conforme (134), em

$$
\begin{aligned}
& E[Z \mid y]=\nu_{e} \frac{\operatorname{Pr}\left\{\frac{\chi_{\nu_{e}+2}^{2}}{\chi_{\nu_{g}}^{2}}>\frac{\nu_{e} m_{e}}{\nu_{g} m_{g}} ; \frac{\chi_{\nu_{g}}^{2}}{\chi_{\nu_{b}}^{2}}>\frac{\nu_{g} m_{g}}{\nu_{b} m_{b}}\right\}}{\operatorname{Pr}\left\{\frac{\chi_{\nu_{g}}^{2}}{\chi_{\nu_{g}}^{2}}>\frac{\nu_{e} m_{g}}{\nu_{g} m_{g}} ; \frac{\chi_{\nu_{g}}^{2}}{\chi_{\nu_{b}}^{2}}>\frac{\nu_{g} m_{g}}{\nu_{b} m_{b}}\right\}}=\nu_{e} \frac{P_{7}}{P_{9}} \quad e \\
& E\left[Z^{2} \mid \boldsymbol{y}\right]=\nu_{e}\left(\nu_{e}+2\right) \frac{\operatorname{Pr}\left\{\frac{\chi_{\nu_{g}+4}^{2}}{\chi_{\nu_{g}}^{2}}>\frac{\nu_{e} m_{g}}{\nu_{g} m_{g}} ; \frac{\chi_{\nu_{g}}^{2}}{\chi_{\nu_{b}}^{2}}>\frac{\nu_{g} m_{g}}{\nu_{b} m_{b}}\right\}}{\operatorname{Pr}\left\{\frac{\chi_{\nu_{e}}^{2}}{\chi_{\nu_{g}}^{2}}>\frac{\nu_{s} m_{e}}{\nu_{g} m_{g}}: \frac{\chi_{\nu_{g}}^{2}}{\chi_{\nu_{b}}^{2}}>\frac{\nu_{g} m_{g}}{\nu_{b} m_{b}}\right\}}=\nu_{e}\left(\nu_{e}+2\right) \frac{P_{3}}{P_{9}},
\end{aligned}
$$

respectivamente.

Cálculo de $e$ e $f$

Equacionando os dois primeiros momentos de $Z$ com os dois primeiros momentos da variável $e \chi_{f}^{2}$, encontra-se

$$
f=\nu_{e}^{*}=\frac{\nu_{e}}{e} \frac{P_{7}}{P_{9}} \quad e=\frac{\left(\nu_{e}+2\right) P_{8}}{2 P_{\tau}}-\frac{\nu_{e} P_{7}}{2 P_{9}}
$$


Agora,

$$
Z=\frac{\nu_{e} m_{\epsilon}}{\sigma_{e}^{2}} \dot{\sim} e \chi_{f}^{2} \Longrightarrow \sigma_{e}^{2} \dot{\sim} \frac{\nu_{e} m_{e}}{e} \chi_{f}^{-2} \Longrightarrow\left\{\begin{array}{l}
f=\nu_{e}^{*} \\
\frac{\nu_{e} m_{e}}{e}=\nu_{e}^{*} m_{e}^{*} \Longrightarrow m_{e}^{*}=\frac{\nu_{e} m_{e}}{e \nu_{e}^{*}}
\end{array}\right.
$$

Assim, uma aproximação para a distribuição marginal posteriori de $\sigma_{e}^{2}$, será dada por

$$
p\left(\sigma_{e}^{2} \mid \boldsymbol{y}\right) \doteq\left(\nu_{e}^{*} m_{e}^{*}\right)^{-1} p\left(\chi_{\nu_{e}^{*}}^{-2}=\frac{\sigma_{e}^{2}}{\nu_{e}^{*} m_{e}^{*}}\right) I_{(0, \infty)}\left(\sigma_{e}^{2}\right)
$$

Intervalos de credibilidade aproximados para $\sigma_{e}^{2}$, podem ser feitos usando o fato de que

$$
\frac{\nu_{e}^{*} m_{e}^{*}}{\sigma_{e}^{2}} \dot{\sim} \chi_{\nu_{e}^{*}}^{2}
$$

ou seja, para $0<\eta_{1}<\eta_{2}<\infty$,

$$
\operatorname{Pr}\left\{\frac{\nu_{e}^{*} m_{e}^{*}}{\eta_{2}} \leq \sigma_{e}^{2} \leq \frac{\nu_{e}^{*} m_{e}^{*}}{\eta_{1}}\right\} \doteq 1-\alpha
$$

onde $\eta_{1}$ e $\eta_{2}$ são valores obtidos das tabelas usuais da distribuição de qui-quadrado $\operatorname{com} \nu_{e}^{*}$ graus de liberdade.

O cálculo de $(e, f)$ envolve uma integral de Dirichlet bivariada, a qual pode ser resolvida usando a identidade dada em (85). Embora esta simplifique o problema, existem métodos de aproximação para tal integral, como pode ser visto em Tiao \& GutTMan (1965).

\section{Uma aproximação pela $\chi^{-2}$ em dois estágios}

Além da aproximação para a distribuição posteriori de $\sigma_{e}^{2}$ dada em (139), BOX \& TIAO (1973) propõem uma outra aproximação, a qual é feita em dois estágios: primeiro considera-se a distribuição conjunta posteriori de $\left(\sigma_{e}^{2}, \sigma_{e g}^{2}\right)$, a qual é obtida da integração de $p\left(\sigma_{e}^{2}, \sigma_{e g}^{2}, \sigma_{e g b}^{2} \mid \boldsymbol{y}\right)$, dada em (89), em relação a $\sigma_{e g b}^{2}$, ou seja,

$$
\begin{aligned}
p\left(\sigma_{e}^{2}, \sigma_{e g}^{2} \mid \boldsymbol{y}\right)= & \delta\left(\nu_{e} m_{e}\right)^{-1} p\left(\chi_{\nu_{e}}^{-2}=\frac{\sigma_{e}^{2}}{\nu_{e} m_{e}}\right)\left(\nu_{g} m_{g}\right)^{-1} p\left(\chi_{\nu_{g}}^{-2}=\frac{\sigma_{e g}^{2}}{\nu_{g} m_{g}}\right) \\
& \frac{\nu_{b} m_{b}}{\Gamma\left(\frac{\nu_{b}}{2}\right) 2^{\frac{\nu_{b}}{2}}} \int_{\sigma_{e g}}^{\infty}\left(\frac{\sigma_{e g b}^{2}}{\nu_{b} m_{b}}\right)^{-\left(\frac{\nu_{b}}{2}+1\right)} \exp \left\{-\frac{\nu_{b} m_{b}}{2 \sigma_{e g b}^{2}}\right\} d \sigma_{e g b}^{2},
\end{aligned}
$$


a qual resulta em

$$
p\left(\sigma_{e}^{2}, \sigma_{e g}^{2} \mid \boldsymbol{y}\right)=\delta^{\prime}\left(\nu_{e} m_{e}\right)^{-1} p\left(\chi_{\nu_{e}}^{-2}=\frac{\sigma_{e}^{2}}{\nu_{e} m_{e}}\right) G_{2}\left(\sigma_{e g}^{2}\right), \quad \sigma_{e g}^{2}>\sigma_{e}^{2}>0
$$

onde

$$
G_{2}\left(\sigma_{e g}^{2}\right)=\frac{\left(\nu_{g} m_{g}\right)^{-1} p\left(\chi_{\nu_{g}}^{-2}=\frac{\sigma_{s g}^{2}}{\nu_{g} m_{g}}\right) \operatorname{Pr}\left\{\chi_{\nu_{b}}^{2}<\frac{\nu_{b} m_{b}}{\sigma_{g g}^{2}}\right\}}{\operatorname{Pr}\left\{F_{\nu_{b}, \nu_{g}}^{2}<\frac{m_{b}}{m_{g}}\right\}}
$$

e

$$
\delta^{\prime}=\delta P r\left\{F_{\nu_{b}, \nu_{3}}<\frac{m_{b}}{m_{g}}\right\} .
$$

Considerando-se a função $G_{2}\left(\sigma_{e g}^{2}\right)$ como se fosse a distribuição de $\sigma_{\varepsilon g}^{2}$, pode-se entāo, empregar o método da aproximaçào por uma simples $\chi^{-2}$, conforme foi desenvolvido anteriormente, isto é, $\sigma_{e g}^{2} \dot{\sim} \nu_{g}^{\prime} m_{g}^{\prime} \chi_{\nu_{g}^{\prime}}^{-2}$.

Equacionando-se os dois primeiros momentos da variável $Z=\frac{\nu_{g} m_{g}}{\sigma_{\varepsilon g}^{2}}$ com os dois primeiros momentos de $a \chi_{b}^{2}$, obtem-se

$$
b=\frac{\nu_{g} P_{1}}{a P_{3}} \quad \in \quad a=\frac{\left(\nu_{g}+2\right)}{2} \frac{P_{2}}{P_{1}}-\frac{\nu_{g}}{2} \frac{P_{1}}{P_{3}}
$$

Agora.

$$
Z=\frac{\nu_{g} m_{g}}{\sigma_{e g}^{2}} \dot{\sim} a \chi_{b}^{2} \Longrightarrow \sigma_{e g}^{2} \dot{\sim} \frac{\nu_{g} m_{g}}{a} \chi_{b}^{-2} \Longrightarrow\left\{\begin{array}{l}
b=\nu_{g}^{\prime} \\
\frac{\nu_{g} m_{g}}{a}=\nu_{g}^{\prime} m_{g}^{\prime} \Longrightarrow m_{g}^{\prime}=\frac{\nu_{g} m_{g}}{a \nu_{g}^{\prime}}
\end{array}\right.
$$

onde

$$
\begin{gathered}
P_{1}=\operatorname{Pr}\left\{F_{\nu_{b}, \nu_{g}+2}<\left(\frac{\nu_{g}+2}{\nu_{g}}\right) \frac{m_{b}}{m_{g}}\right\}=I_{y}\left(\frac{\nu_{b}}{2}, \frac{\nu_{g}}{2}+1\right), \\
P_{2}=\operatorname{Pr}\left\{F_{\nu_{b}, \nu_{g}+4}<\left(\frac{\nu_{g}+4}{\nu_{g}}\right) \frac{m_{b}}{m_{g}}\right\}=I_{y}\left(\frac{\nu_{b}}{2}, \frac{\nu_{g}}{2}+2\right), \\
P_{3}=\operatorname{Pr}\left\{F_{\nu_{b}, \nu_{g}}<\frac{m_{b}}{m_{g}}\right\}=I_{y}\left(\frac{\nu_{b}}{2}, \frac{\nu_{g}}{2}\right) \quad \text { e } y=\frac{\nu_{b} m_{b}}{\nu_{b} m_{b}+\nu_{g} m_{g}} .
\end{gathered}
$$

Agora, a expressão (142) pode ser escrita como

$$
\begin{aligned}
p\left(\sigma_{e:}^{2} \sigma_{e g}^{2} \mid y\right) \doteq & \delta^{\prime}\left(\nu_{e} m_{e}\right)^{-1} p\left(\chi_{\nu_{s}}^{-2}=\frac{\sigma_{e}^{2}}{\nu_{e} m_{e}}\right)\left(\nu_{g}^{\prime} m_{g}^{\prime}\right)^{-1} p\left(\chi_{\nu_{g}^{\prime}}^{-2}=\frac{\sigma_{e g}^{2}}{\nu_{g}^{\prime} m_{g}^{\prime}}\right), \\
& \sigma_{e g}^{2}>\sigma_{e}^{2}>0 .
\end{aligned}
$$


Observa-se que o efeito da integração, modifica o quadrado médio da interação, de $m_{g}$ para $m_{g}^{\prime}$ e os graus de liberdade, de $\nu_{g}$ para $\nu_{g}^{\prime}$.

Por outrolado, sabendo-se que $\sigma_{e g}^{2}=\sigma_{e}^{2}+R \sigma_{g}^{2}$, então, a distribuição posteriori de $\left(\sigma_{e}^{2}, \sigma_{g}^{2}\right)$ pode ser expressa como

$$
\begin{aligned}
p\left(\sigma_{e}^{2}, \sigma_{g}^{2} \mid \boldsymbol{y}\right) \doteq \frac{\delta^{\prime \prime} R\left(\nu_{e} m_{e}\right)^{-1} p\left(\chi_{\nu_{e}}^{-2}=\frac{\sigma_{e}^{2}}{\nu_{e} m_{e}}\right)\left(\nu_{g}^{\prime} m_{g}^{\prime}\right)^{-1} p\left(\chi_{\nu_{g}^{\prime}}^{-2}=\frac{\sigma_{e g}^{2}}{\nu_{g}^{\prime} m_{g}^{\prime}}\right)}{\operatorname{Pr}\left\{F_{\nu_{g}^{\prime}, \nu_{e}}<\frac{m_{g}^{\prime}}{m_{e}}\right\}} \\
\sigma_{e g}^{2}>\sigma_{e}^{2}>0
\end{aligned}
$$

onde

$$
\delta "=\delta^{\prime} \operatorname{Pr}\left\{F_{\nu_{g}^{\prime}, \nu_{e}}<\frac{m_{g}^{\prime}}{m_{e}}\right\} .
$$

O segundoestágio, também consiste em aproximar $p\left(\sigma_{e}^{2} \mid \boldsymbol{y}\right)$ por uma simples $\chi^{-2}$. Isto é, $\sigma_{e}^{2} \dot{\sim} \nu_{e}^{\prime} m_{e}^{\prime} \chi_{\nu_{e}^{\prime}}^{-2}$, onde $\nu_{e}^{\prime}$ e $m_{e}^{\prime}$ serão determinados conforme se segue.

$$
\nu_{e}^{\prime}=\frac{\nu_{e}}{k} \frac{P_{10}}{P_{12}} \quad e \quad k=\frac{\left(\nu_{e}+2\right)}{2} \frac{P_{11}}{P_{10}}-\frac{\nu_{e}}{2} \frac{P_{10}}{P_{12}}, \quad m_{e}^{\prime}=\frac{\nu_{e} m_{e}}{k \nu_{e}^{\prime}}
$$

onde

$$
\begin{gathered}
P_{10}=\operatorname{Pr}\left\{F_{\nu_{g}^{\prime}, \nu_{e}+2}<\left(\frac{\nu_{e}+2}{\nu_{e}}\right) \frac{m_{g}^{\prime}}{m_{e}}\right\}=I_{x}\left(\frac{\nu_{g}^{\prime}}{2}, \frac{\nu_{e}}{2}+1\right), \\
P_{11}=\operatorname{Pr}\left\{F_{\nu_{g}^{\prime}, \nu_{e}+4}<\left(\frac{\nu_{e}+4}{\nu_{e}}\right) \frac{m_{g}^{\prime}}{m_{e}}\right\}=I_{x}\left(\frac{\nu_{g}^{\prime}}{2}, \frac{\nu_{e}}{2}+2\right), \\
P_{12}=\operatorname{Pr}\left\{F_{\nu_{g}^{\prime}, \nu_{e}}<\frac{m_{g}^{\prime}}{m_{e}}\right\}=I_{x}\left(\frac{\nu_{g}^{\prime}}{2}, \frac{\nu_{e}}{2}\right) \quad \text { e } x=\frac{\nu_{g}^{\prime} m_{g}^{\prime}}{\nu_{g}^{\prime} m_{g}^{\prime}+\nu_{e} m_{e}} .
\end{gathered}
$$

Para este grau de aproximação, a densidade posteriori de $\sigma_{e}^{2}$ aproximada em dois estágios será

$$
p\left(\sigma_{e}^{2} \mid y\right) \doteq\left(\nu_{e}^{\prime} m_{e}^{\prime}\right)^{-1} p\left(\chi_{\nu_{e}^{\prime}}^{-2}=\frac{\sigma_{e}^{2}}{\nu_{e}^{\prime} m_{e}^{\prime}}\right) I_{(0, \infty)}\left(\sigma_{e}^{2}\right) .
$$

Se o efeito da restrição $C: \sigma_{e g b}^{2}>\sigma_{e g}^{2}>\sigma_{e}^{2}$ for ignorado (ou desprezível), então inferências sobre $\sigma_{e}^{2}$ podem ser feitas a partir da distribuição irrestrita, ou seja,

$$
p\left(\sigma_{e}^{2} \mid \boldsymbol{y}\right)=\left(\nu_{e} m_{e}\right)^{-1} p\left(\chi_{\nu_{e}}^{-2}=\frac{\sigma_{e}^{2}}{\nu_{e} m_{e}}\right) I_{(0, \infty)}\left(\sigma_{e}^{2}\right)
$$


Probabilidades posteriori acerca de $\sigma_{e}^{2}$ podem ser determinadas com base no fato de que

$$
\frac{\nu_{e} m_{e}}{\sigma_{e}^{2}} \sim \chi_{\nu_{e}}^{2}
$$

e para $0<\eta_{1}<\eta_{2}<\infty$,

$$
\operatorname{Pr}\left\{\frac{\nu_{e} m_{e}}{\eta_{2}} \leq \sigma_{e}^{2} \leq \frac{\nu_{e}^{\prime} m_{e}}{\eta_{1}}\right\}=1-\alpha
$$

onde $\eta_{1}$ e $\eta_{2}$ são obtidos das tabelas usuais da distribuição de $\chi^{2}$.

\subsubsection{Composição das Estimativas das Variâncias para Estimar $\sigma_{e}^{2}$}

A aproximação por uma $\chi^{-2}$ simples em dois estágios, para a distribuição posteriori de $\sigma_{e}^{2}$, dada em (151), pode, alternativamente, ser posta na forma

$$
\frac{1}{\sigma_{e}^{2}} \frac{\nu_{e} m_{e}+\lambda_{1} \nu_{g} m_{g}+\lambda_{2} \nu_{b} m_{b}}{\nu_{e}+w_{1} \nu_{g}+w_{2} \nu_{b}} \dot{\sim} \frac{\chi_{\left(\nu_{s}+w_{1} \nu_{g}+w_{2} \nu_{b}\right)}^{2}}{\nu_{e}+w_{1} \nu_{g}+w_{2} \nu_{b}}
$$

onde

$$
\begin{aligned}
& \lambda_{1}=\left(\frac{1}{k}-1\right) \frac{\nu_{e} m_{\varepsilon}}{\nu_{g}^{\prime} m_{g}^{\prime}}, \quad w_{1}=\frac{\nu_{g}^{\prime}-\nu_{e}}{\nu_{g}^{\prime}} \\
& \lambda_{2}=\left(\frac{1}{k}-1\right)(1-a) \frac{\nu_{e} m_{s}}{\nu_{b} m_{b}} \quad \text { e } w_{2}=\left(\frac{\nu_{s}^{\prime}-\nu_{s}}{\nu_{b}}\right)\left(1-\frac{\nu_{q}}{\nu_{g}^{\prime}}\right) .
\end{aligned}
$$

Conforme Box \& TiaO (1973), a expressão (154), pode ser pensada como a solução bayesiana fornecida pela composição dos três quadrados médios $\left(m_{e}, m_{g}, m_{b}\right)$ para estimar o componente da variância $\sigma_{e}^{2}$.

Para calcular probabilidades posteriori acerca da variância composta deve ser usado o fato indicado na expressão (154).

\subsubsection{Distribuição Marginal a Posteriori de $\sigma_{g}^{2}$}

A distribuição posteriori de $\sigma_{g}^{2}$ é obtida, aproximadamente, integrando-se $p\left(\sigma_{e}^{2}, \sigma_{g}^{2} \mid \boldsymbol{y}\right)$, dada em (148), em relação a $\sigma_{e}^{2}$, isto é,

$$
\begin{aligned}
p\left(\sigma_{g}^{2} \mid \boldsymbol{y}\right) \doteq & \delta^{\prime \prime} \frac{R\left(\nu_{e} m_{e}\right)^{-1}\left(\nu_{g}^{\prime} m_{g}^{\prime}\right)^{-1}}{\operatorname{Pr}\left\{F_{\nu_{g}^{\prime}, \nu_{e}}<\frac{m_{g}^{\prime}}{m_{e}}\right\}} \int_{0}^{\infty} p\left(\chi_{\nu_{e}}^{-2}=\frac{\sigma_{e}^{2}}{\nu_{e} m_{e}}\right) p\left(\chi_{\nu_{g}^{\prime}}=\frac{\sigma_{e}^{2}+R \sigma_{g}^{2}}{\nu_{g}^{\prime} m_{g}^{\prime}}\right) d \sigma_{e}^{2} \\
& \sigma_{g}>0
\end{aligned}
$$


Observa-se, portanto, que não é possível expressar a distribuição posteriori de $\sigma_{g}^{2}$ em termos de uma função simples. O valor de $p\left(\sigma_{g}^{2} \mid \boldsymbol{y}\right)$, para cada valor de $\sigma_{g}^{2}$, pode ser obtido através de integração numérica, para qualquer precisão desejada. Isto poderá ser feito para avaliar a distribuição posteriori exata de $\sigma_{g}^{2}$. Contudo, aproximações menos laboriosas poderão ser usadas. Dentre elas, Box \& TIAO (1973), sugerem que a distribuição posteriori de $\sigma_{g}^{2}$ seja aproximada por

$$
p\left(\sigma_{g}^{2} \mid y\right) \doteq \frac{R\left(\nu_{g}^{\prime} m_{g}^{\prime}\right)^{-1} p\left(\chi_{\nu_{g}^{\prime}}^{-2}=\frac{m_{e}+R \sigma_{g}^{2}}{\nu_{g}^{\prime} m_{g}^{\prime}}\right)}{\operatorname{Pr}\left\{\chi_{\nu_{g}^{\prime}}^{2}<\frac{\nu_{g}^{\prime} m_{g}^{\prime}}{m_{e}}\right\}} I_{(0, \infty)}\left(\sigma_{g}^{2}\right) .
$$

Para este grau de aproximação, a quantidade $\frac{m_{e}+R \sigma_{g}^{2}}{\nu_{g}^{\prime} m_{g}^{\prime}} \dot{\sim} \chi_{\nu_{g}^{\prime}}^{-2}$ truncada abaixo de $\frac{m_{e}}{\nu \frac{\nu_{g}^{\prime}}{m_{g}^{\prime}}}$, ou equivalentemente, a quantidade $\frac{\nu_{g}^{\prime} m_{g}^{\prime} m_{g}^{\prime}}{m_{e}+R \sigma_{g}^{2}} \dot{\sim} \chi_{\nu_{g}^{\prime}}^{2}$ truncada acima de $\frac{\nu_{g}^{\prime} m_{g}^{\prime}}{m_{e}}$.

Probabilidades posteriori acerca de $\sigma_{g}^{2}$, podem ser aproximadamente determinadas, usando-se as tabelas usuais da distribuição de $\chi^{2}$. Neste sentido, para $0<\eta_{1}<\eta_{2}<\infty$, tem-se que

$$
\operatorname{Pr}\left\{\eta_{1}<\sigma_{g}^{2}<\eta_{2} \mid y\right\} \doteq \frac{\operatorname{Pr}\left\{\frac{\nu_{g}^{\prime} m_{g}^{\prime}}{m_{e}+R \eta_{2}}<\chi_{\nu_{g}^{\prime}}^{2}<\frac{\nu_{g}^{\prime} m_{g}^{\prime}}{m_{e}+R \eta_{1}}\right\}}{\operatorname{Pr}\left\{\chi_{\nu_{g}^{\prime}}^{2}<\frac{\nu_{g}^{\prime} m_{g}^{\prime}}{m_{e}}\right\}} .
$$

Em particular, para $\eta>0$, tem-se que

$$
\operatorname{Pr}\left\{0<\sigma_{\boldsymbol{g}}^{2}<\eta \mid \boldsymbol{y}\right\} \doteq \frac{\operatorname{Pr}\left\{\frac{\nu_{g}^{\prime} m_{g}^{\prime}}{m_{e}+R \eta}<\chi_{\nu_{g}^{\prime}}^{2}<\frac{\nu_{g}^{\prime} m_{g}^{\prime}}{m_{e}}\right\}}{\operatorname{Pr}\left\{\chi_{\nu_{g}^{\prime}}^{2}<\frac{\nu_{g}^{\prime} m_{g}^{\prime}}{m_{e}}\right\}} .
$$

\subsubsection{Distribuição Marginal a Posteriori de $\sigma_{b}^{2}$}

A densidade posteriori de $\sigma_{b}^{2}, p\left(\sigma_{b}^{2} \mid \boldsymbol{y}\right)$, pode ser obtida, integrandose $p\left(\sigma_{e}^{2}, \sigma_{g}^{2}, \sigma_{b}^{2} \mid \boldsymbol{y}\right)$, dada em (92), em relação a $\sigma_{e}^{2}$ e $\sigma_{g}^{2}$, ou seja,

$$
\begin{aligned}
p\left(\sigma_{b}^{2} \mid \boldsymbol{y}\right)= & \delta\left(K R^{2}\right) \int_{0}^{\infty} \int_{0}^{\infty}\left(\nu_{e} m_{e}\right)^{-1} p\left(\chi_{\nu_{e}}^{-2}=\frac{\sigma_{e}^{2}}{\nu_{e} m_{e}}\right)\left(\nu_{g} m_{g}\right)^{-1} p\left(\chi_{\nu_{g}}^{-2}=\frac{\sigma_{e}^{2}+R \sigma_{g}^{2}}{\nu_{g} m_{g}}\right) \\
& \times\left(\nu_{b} m_{b}\right)^{-1} p\left(\chi_{\nu_{b}}^{-2}=\frac{\sigma_{e}^{2}+R \sigma_{g}^{2}+K R \sigma_{b}^{2}}{\nu_{b} m_{b}}\right) d \sigma_{e}^{2} d \sigma_{g}^{2} \\
& \sigma_{b}^{2}>0 .
\end{aligned}
$$


Observa-se, portanto, que $p\left(\sigma_{b}^{2} \mid \boldsymbol{y}\right)$ nào pode ser expressa em termos de uma funçào simples. Avaliaçōes exatas de $p\left(\sigma_{b}^{2} \mid \boldsymbol{y}\right)$ são, sem dúvida, bastante laboriosas. Em geral, usa-se uma aproximação para ela. Esta é obtida, integrandose inicialmente, $p\left(\sigma_{e}^{2}, \sigma_{e g}^{2}, \sigma_{e g b}^{2} \mid \boldsymbol{y}\right)$, dada em (89), em relaçào a $\sigma_{e}^{2}$, isto é,

$$
\begin{aligned}
p\left(\sigma_{e g}^{2}, \sigma_{e g b}^{2} \mid \boldsymbol{y}\right) & =\int_{0}^{\infty} p\left(\sigma_{e}^{2}, \sigma_{e g}^{2}, \sigma_{e g b}^{2} \mid \boldsymbol{y}\right) d \sigma_{e}^{2} \\
& =\psi\left(\nu_{b} m_{b}\right)^{-1} p\left(\chi_{\nu_{b}}^{-2}=\frac{\sigma_{e g b}^{2}}{\nu_{b} m_{b}}\right) G_{3}\left(\sigma_{e g}^{2}\right), \quad \sigma_{e g b}^{2}>\sigma_{e g}^{2}>0
\end{aligned}
$$

onde

$$
G_{3}\left(\sigma_{e g}^{2}\right)=\frac{\left(\nu_{g} m_{g}\right)^{-1} p\left(\chi_{\nu_{g}}^{-2}=\frac{\sigma_{s g}^{2}}{\nu_{g} m_{g}}\right) \operatorname{Pr}\left\{\chi_{\nu_{s}}^{2}>\frac{\nu_{\varepsilon} m_{e}}{\sigma_{e_{g}}^{2}}\right\}}{\operatorname{Pr}\left\{F_{\nu_{g}, \nu_{e}}<\frac{m_{g}}{m_{s}}\right\}}
$$

e

$$
\psi^{\prime}=\delta \operatorname{Pr}\left\{F_{\nu_{y}, \nu_{e}}<\frac{m_{g}}{m_{e}}\right\} .
$$

Observa-se que $G_{3}\left(\sigma_{e g}^{2}\right)$ em $(162)$, tem a mesma forma de $G_{2}\left(\sigma_{e g}^{2}\right)$ em (143). E, portanto, $p\left(\sigma_{e g}^{2}, \sigma_{e g b}^{2} \mid \boldsymbol{y}\right)$ pode ser aproximada, usando aquela mesma metodologia, por

$$
\begin{aligned}
p\left(\sigma_{e g}^{2}, \sigma_{e g b}^{2} \mid \boldsymbol{y}\right) \doteq & \frac{\left(\nu_{b} m_{b}\right)^{-1} p\left(\chi_{\nu_{b}}^{-2}=\frac{\sigma_{e g b}^{2}}{\nu_{b} m_{b}}\right)\left(\nu_{g}^{\prime \prime} m_{g}{ }^{\prime \prime}\right)^{-1} p\left(\chi_{\nu_{g} "}^{-2}=\frac{\sigma_{\varepsilon g}^{2}}{\nu_{g}^{\prime \prime} m_{g} "}\right)}{\operatorname{Pr}\left\{F_{\nu_{b}, \nu_{g} "}{ }^{\prime \prime}<\frac{m_{b}}{m_{g}}\right\}} \\
& \sigma_{e g b}^{2}>\sigma_{e g}^{2}>0,
\end{aligned}
$$

onde

$$
\begin{gathered}
\nu_{g}{ }^{\prime}=\frac{\nu_{g}}{t} \frac{P_{13}}{P_{15}}, \quad t=\frac{\left(\nu_{g}+2\right)}{2} \frac{P_{14}}{P_{13}}-\frac{\nu_{g}}{2} \frac{P_{13}}{P_{15}}, \quad m_{g}{ }^{\prime}=\frac{\nu_{g} m_{g}}{t \nu_{g}{ }^{\prime}}, \\
P_{13}=\operatorname{Pr}\left\{F_{\nu_{g}+2, \nu_{e}}<\left(\frac{\nu_{g}}{\nu_{g}+2}\right) \frac{m_{e}}{m_{g}}\right\}=I_{x}\left(\frac{\nu_{g}}{2}+1, \frac{\nu_{e}}{2}\right): \\
P_{14}=\operatorname{Pr}\left\{F_{\nu_{g}+4, \nu_{s}}<\left(\frac{\nu_{g}}{\nu_{g}+4}\right) \frac{m_{e}}{m_{g}}\right\}=I_{x}\left(\frac{\nu_{g}}{2}+2, \frac{\nu_{e}}{2}\right), \\
P_{15}=\operatorname{Pr}\left\{F_{\nu_{g}, \nu_{s}}<\frac{m_{g}}{m_{e}}\right\}=I_{x}\left(\frac{\nu_{g}}{2}, \frac{\nu_{e}}{2}\right) \quad e \quad x=\frac{\nu_{g} m_{g}}{\nu_{g} m_{g}+\nu_{e} m_{e}} .
\end{gathered}
$$

Lembrando que $\sigma_{e g b}^{2}=\sigma_{\epsilon g}^{2}+K R \sigma_{b}^{2}$, cujo jacobiano da transformaçào é $K R$. entào a densidade a posteriori de $\left(\sigma_{e g}^{2}, \sigma_{b}^{2}\right)$ será dada, aproximadamente, por

$$
p\left(\sigma_{\varepsilon}^{2}, \sigma_{b}^{2} \mid y\right)
$$




$$
\begin{aligned}
\doteq & \frac{K R\left(\nu_{b} m_{b}\right)^{-1}\left(\nu_{g} " m_{g}{ }^{\prime}\right)^{-1}}{\operatorname{Pr}\left\{F_{\nu_{b}, \nu_{g}}{ }^{\prime}<\frac{m_{b}}{m_{g}}\right\}} p\left(\chi_{\nu_{b}}^{-2}=\frac{\sigma_{e g}^{2}+K R \sigma_{b}^{2}}{\nu_{b} m_{b}}\right) p\left(\chi_{\nu_{g} "}^{-2}=\frac{\sigma_{e g}^{2}}{\nu_{g}{ }^{\prime \prime} m_{g}{ }^{\prime \prime}}\right) \\
& \sigma_{e g}^{2}>0, \quad \sigma_{b}^{2}>0
\end{aligned}
$$

e segue, portanto, que a distribuição marginal posteriori de $\sigma_{b}^{2}$, será dada, aproximadamente. por

$$
\begin{aligned}
& p\left(\sigma_{b}^{2} \mid y\right) \\
& \doteq \frac{K R\left(\nu_{b} m_{b}\right)^{-1}\left(\nu_{g} " m_{g}{ }^{\prime \prime}\right)^{-1}}{\operatorname{Pr}\left\{F_{\nu^{\prime}, \nu_{g}} "<\frac{m_{b}}{m_{g}}\right\}} \int_{0}^{\infty} p\left(\chi_{\nu_{b}}^{-2}=\frac{\sigma_{e g}^{2}+K R \sigma_{b}^{2}}{\nu_{b} m_{b}}\right) p\left(\chi_{\nu_{g}}^{-2}=\frac{\sigma_{e g}^{2}}{\nu_{g} " m_{g} "}\right) d \sigma_{e g}^{2} \text {; } \\
& \sigma_{b}^{2}>0
\end{aligned}
$$

Observa-se que $p\left(\sigma_{b}^{2} \mid \boldsymbol{y}\right)$, em (167), tem exatamente a mesma forma de $p\left(\sigma_{g}^{2} \mid \boldsymbol{y}\right)$, dada em (156). Neste caso, a sugestão é aproximar $p\left(\sigma_{b}^{2} \mid \boldsymbol{y}\right)$ usando a mesma técnica que foi usada para aproximar $p\left(\sigma_{g}^{2} \mid \boldsymbol{y}\right)$, isto é,

$$
p\left(\sigma_{b}^{2} \mid \boldsymbol{y}\right) \doteq \frac{K R\left(\nu_{b} m_{b}\right)^{-1} p\left(\chi_{\nu_{b}}^{-2}=\frac{m_{g} "+K R \sigma_{b}^{2}}{\nu_{b} m_{b}}\right)}{\operatorname{Pr}\left\{\chi_{\nu_{b}}^{2}<\frac{\nu_{b} m_{b}}{m_{g}^{\prime \prime}}\right\}} I_{(0, \infty)}\left(\sigma_{b}^{2}\right)
$$

Para esta aproximação, a quantidade $\frac{m_{g} "+K R \sigma_{b}^{2}}{\nu_{b} m_{b}} \dot{\sim} \chi_{\nu_{b}}^{-2}$ truncada abaixo de $\frac{m_{g}{ }^{\prime \prime}}{\nu_{b} m_{b}}$, ou equivalentemente, a quantidade $\frac{\nu_{b} m_{b}}{m_{g} "+K R \sigma_{b}^{2}} \dot{\sim} \chi_{\nu_{b}}^{2}$ truncada acima de $\frac{\nu_{b} m_{b}}{m_{g}}$. Probabilidades posteriori acerca de $\sigma_{b}^{2}$, podem ser determinadas aproximadamente, empregando-se as tabelas usuais da distribuição de $\chi^{2}$.

$$
\begin{gathered}
\text { Para } 0<\eta_{1}<\eta_{2}<\infty, \\
\operatorname{Pr}\left\{\eta_{1}<\sigma_{b}^{2}<\eta_{2} \mid y\right\} \doteq \frac{\operatorname{Pr}\left\{\frac{\nu_{b} m_{b}}{m_{3} "+K R \eta_{2}}<\chi_{\nu_{b}}^{2}<\frac{\nu_{b} m_{b}}{m_{g}{ }^{\prime \prime}+K R \eta_{1}}\right\}}{\operatorname{Pr}\left\{\chi_{\nu_{b}}^{2}<\frac{\nu_{b} m_{k}}{m_{g}{ }^{\prime \prime}}\right\}}
\end{gathered}
$$

Em particular, par $\eta>0$, tem-se

$$
\operatorname{Pr}\left\{0<\sigma_{b}^{2}<\eta \mid y\right\} \doteq \frac{\operatorname{Pr}\left\{\frac{\nu_{b} m_{b}}{m_{g} "+K R \eta}<\chi_{\nu_{b}}^{2}<\frac{\nu_{b} m_{b}}{m_{g}{ }^{\prime \prime}}\right\}}{\operatorname{Pr}\left\{\chi_{\nu_{b}}^{2}<\frac{\nu_{b} m_{b}}{m_{g} "}\right\}}
$$




\subsection{Resumo da Teoria}

Dadas as suposições e os dados, tudo que pode ser dito acerca dos paràmetros de interesse, estará nas densidades a posteriori desses parâmetros.

\subsubsection{Análises Individuais}

Sob a luz de uma priori não-informativa de referència, as densidades marginais a posteriori dos paràmetros dos modelos estatísticos associados aos experimentos individuais foram determinadas e encontram-se a seguir.

3.4.1.1 Densidade a Posteriori de $\sigma^{2}, p\left(\sigma^{2} \mid \boldsymbol{y}\right)$

$$
\begin{gathered}
p\left(\sigma^{2} \mid \boldsymbol{y}\right)=\frac{\left(\nu s^{2}\right)^{-1}}{\Gamma\left(\frac{\nu}{2}\right) 2^{\frac{\nu}{2}}}\left(\frac{\sigma^{2}}{\nu s^{2}}\right)^{-\left(\frac{\nu}{2}+1\right)} e^{-\frac{\nu s^{2}}{2 \sigma^{2}}} I_{(0, \infty)}\left(\sigma^{2}\right) . \\
E\left[\sigma^{2} \mid \boldsymbol{y}\right]=\frac{\nu s^{2}}{\nu-1}, \quad \operatorname{Var}\left[\sigma^{2} \mid \boldsymbol{y}\right]=\frac{2\left(\nu s^{2}\right)^{2}}{(\nu-2)^{2}(\nu-1)}, \quad M o\left[\sigma^{2} \mid \boldsymbol{y}\right]=\frac{\nu s^{2}}{\nu+2}, \\
\int_{0}^{\left.M d\left|\sigma^{2}\right| \boldsymbol{y}\right]} p\left(\sigma^{2} \mid \boldsymbol{y}\right) d \sigma^{2}=0,5 \quad e \operatorname{Pr}\left\{\frac{\nu s^{2}}{\chi_{[\nu, S]}^{2}} \leq \sigma^{2} \leq \frac{\nu s^{2}}{\chi_{[\nu, I]}^{2}} \mid \boldsymbol{y}\right\}=1-\alpha,
\end{gathered}
$$

3.4.1.2 Densidade Marginal a Posteriori de $\boldsymbol{\theta}, p(\boldsymbol{\theta} \mid \boldsymbol{y})$

$$
\begin{gathered}
\boldsymbol{\theta} \mid \boldsymbol{y} \sim t_{K}\left[\hat{\boldsymbol{\theta}},\left(X^{\prime} X\right)^{-1} s^{2}, \nu\right], \quad e \quad \theta_{i} \sim t_{1}\left[\hat{\theta}_{i}, \frac{s^{2}}{R}, \nu\right], \\
\hat{\boldsymbol{\theta}}=\left(\hat{\theta}_{1}, \cdots, \hat{\theta}_{K}\right)^{\prime}, \quad \hat{\theta}_{i}=\bar{y}_{i,}, \quad s^{2}=\text { QM.Res., } \quad \nu=K(R-1) \quad e \\
\operatorname{Pr}\left\{\hat{\theta}_{i}-t_{\frac{\alpha}{2}} \sqrt{\left.\frac{s^{2}}{R} \leq \theta_{i} \leq \hat{\theta}_{i}+t_{\frac{\alpha}{2}} \sqrt{\frac{s^{2}}{R}} \mid \boldsymbol{y}\right\}=1-\alpha .}\right.
\end{gathered}
$$

3.4.1.3 Densidade Marginal a Posteriori de $\phi=D \theta=\left(\phi_{i}, \cdots, \phi_{(K-1)}\right)$,

Seja $\boldsymbol{\phi}=D \boldsymbol{\theta}=\left(\phi_{1}, \cdots, \phi_{(K-1)}\right)^{\prime}$, um conjunto de $(K-1)$ contrastes independentes de $\boldsymbol{\theta}$, onde $D=\left(d_{j i}\right), i=1, \cdots, K ; j=1, \cdots,(K-1)$, então, 


$$
\phi \mid y \sim t_{(K-1)}\left[\hat{\phi}, s^{2}\left[D\left(X^{\prime} X\right)^{-1} D^{\prime}\right], \nu\right]
$$

Para comparação geral de que todas as médias dos tratamentos são iguais. regiões H.P.D. para $\phi$ com conteúdo $(1-\alpha)$, serão obtidas por

$$
\frac{S(\phi)}{(K-1) s^{2}} \leq F_{[(K-1), \nu, \alpha]} \text {. }
$$

Em particular, o ponto $\phi_{0}=0$ (isto é, $\theta_{1}=, \cdots,=\theta_{K}$ ) estará na região H.P.D. de conteúdo $(1-\alpha)$, se e somente se,

$$
\frac{S\left(\phi_{0}\right)}{(K-1) s^{2}}=\frac{S(\mathbf{0})}{(K-1) s^{2}}=\frac{R \sum_{i=1}^{K}\left(\bar{y}_{i .}-\bar{y}_{. .}\right)^{2}}{(K-1) s^{2}} \leq F_{[(K-1), \nu, \alpha]} .
$$

Probabilidades marginais a posteriori, para qualquer contraste $\phi_{j}$, podem ser calculadas com base no fato de que

$$
\phi_{j} \mid y \sim t_{1}\left[\hat{\phi}_{j}, s^{2} h_{j j}, \nu\right], \quad h_{j j}=\frac{1}{R} \sum_{i=1}^{K} d_{j i}^{2} .
$$

e

$$
\operatorname{Pr}\left\{\hat{\phi}_{j}-t_{\frac{\alpha}{2}} \sqrt{h_{j j}} \leq \phi_{j} \leq \hat{\phi}_{j}+t \frac{\alpha}{2} \sqrt{h_{j j}} \mid \boldsymbol{y}\right\}=1-\alpha
$$

\subsubsection{Comparação das $K$ Dispersões}

Para verificar a homogeneidade das $K$ variâncias, são estabelecidos $(K-1)$ contrastes do tipo

$$
\varphi_{i}=\log \sigma_{i}^{2}-\log \sigma_{K}^{2}, \quad i=1, \cdots,(K-1) .
$$

Os dois testes usados, se baseiam na distribuição conjunta a posteriori de $\varphi=\left(\varphi_{1}, \cdots, \varphi(K-1)\right)^{\prime}$.

Em particular, o ponto $\varphi_{0}=0$, (isto é, $\sigma_{1}^{2}=, \cdots,=\sigma_{K}^{2}$ ), estará na região H.P.D. de conteúdo $(1-\alpha)$ se, e somente se,

$$
\frac{-2 \log W_{0}}{1+A} \leq \chi_{[(K-1), \alpha]}^{2}, \quad \text { (Teste de Bartlett Modificado) }
$$


ou

$$
\sum_{i=1}^{K} \frac{\left(m_{i}-m\right)^{2}}{a_{i}^{2}} \leq \chi_{[(K-1), \alpha]}^{2}, \quad \text { (Teste da Raiz Cúbica). }
$$

Utilizar as expressões dadas em (58).

\subsubsection{A Análise Conjunta}

Uma vez comprovada a homocedasticidade das variâncias dentro e entre os experimentos, a análise conjunta poderá ser realizada e as densidades marginais poderão ser determinadas.

3.4.2.1 Densidade Marginal a Posteriori Aproximada de $\phi=\left(\phi_{1}, \cdots, \phi_{(K-1)}\right)^{\prime}$

A distribuição marginal a posteriori aproximada de $\phi$ é dada por

$$
\phi \mid y \quad \dot{\sim} t_{(K-1)}\left[\hat{\phi}, m_{g}^{*}, \nu^{*}\right]
$$

onde $\hat{\boldsymbol{\phi}}=D \boldsymbol{\theta}, D=\left(d_{j i}\right), i=1, \cdots, K ; j=1, \cdots,(K-1)$, é qualquer matriz de $(K-1)$ coeficientes dos contrastes independentes $\operatorname{dos} \boldsymbol{\theta}$ e $\hat{\boldsymbol{\theta}}_{i}=\bar{y}_{i . .}$. As quantidades $m_{g}^{*}$ e $\nu^{*}$ serão calculadas conforme expressões dadas em (106) a (108) e (115) a (118). Ainda, $\sum=\left[D\left(X^{\prime} X\right)^{-1} D^{\prime}\right]=\operatorname{Diag}\left[\sum_{i=1}^{K} d_{j i}^{2}, j=1, \cdots,(K-1)\right]$.

Para comparação geral de que $\theta_{1}=\theta_{2}=\cdots=\theta_{K}$ a região H.P.D. para $\phi \operatorname{com}(1-\alpha)$ é dada por

$$
\frac{S(\phi)}{(K-1) m_{g}^{*}} \leq F_{\left[(K-1), \nu_{g}^{*}, \alpha\right]}
$$

Em particular, o ponto $\phi_{0}=\mathbf{0}$ (isto é, $\theta_{1}=\cdots=\theta_{K}$ ) estará na região H.P.D. de conteúdo $(1-\alpha)$ se, e somente se,

$$
\frac{S\left(\boldsymbol{\phi}_{0}\right)}{(K-1) m_{g}^{*}}=\frac{S(\mathbf{0})}{(K-1) m_{g}^{*}}=\frac{\hat{\boldsymbol{\phi}}^{\prime}\left[D\left(X^{\prime} X\right)^{-1} D^{\prime}\right] \hat{\boldsymbol{\phi}}}{(K-1) m_{g}^{*}}=\frac{\text { S.Q.Trat. }}{(K-1) m_{g}^{*}} \leq F_{\left[(K-1), \nu_{g}^{*}, \alpha\right]}
$$

A distribuição marginal a posteriori de $\phi_{j}, j=1, \cdots,(K-1)$ é dada de modo aproximado por 


$$
\phi_{j} \mid y \dot{\sim} \quad t_{1}\left[\hat{\phi}_{j}, \frac{m_{g}^{*}}{J R} \sum_{i=1}^{K} d_{j i}^{2}, \nu_{g}^{*}\right]
$$

e segue que

$$
\operatorname{Pr}\left\{\dot{\phi}_{j}-t_{\frac{\alpha}{2}} \sqrt{\frac{m_{g}^{*}}{J R} \sum_{i=1}^{K} d_{j i}^{2}} \leq \phi_{j} \leq \hat{\phi}_{j}+t_{\frac{\alpha}{2}} \sqrt{\frac{m_{g}^{*}}{J R} \sum_{i=1}^{K} d_{j i}^{2} \mid y}\right\}=1-\alpha .
$$

3.4.3 Distribuição Marginal a Posteriori dos Componentes da Variância, $\sigma_{e}^{2}, \sigma_{g}^{2}$ e $\sigma_{b}^{2}$

3.4.3.1 Distribuição Marginal a Posteriori Aproximada de $\sigma_{e}^{2}$

$$
p\left(\sigma_{e}^{2} \mid \boldsymbol{y}\right) \doteq\left(\nu_{e}^{\prime} m_{e}^{\prime}\right)^{-1} p\left(\chi_{\nu_{e}^{\prime}}^{-2}=\frac{\sigma_{e}^{2}}{\nu_{e}^{\prime} m_{e}^{\prime}}\right) I_{(0, \infty)}\left(\sigma_{e}^{2}\right),
$$

onde $\nu_{e}^{\prime}$ e $m_{e}^{\prime}$ serão obtidos pelas expressões dadas em (149).

3.4.3.2 Distribuição Marginal a Posteriori Aproximada de $\sigma_{g}^{2}$

$$
p\left(\sigma_{g}^{2} \mid \boldsymbol{y}\right) \doteq \frac{R\left(\nu_{g}^{\prime} m_{g}^{\prime}\right)^{-1} p\left(\chi_{\nu_{g}^{\prime}}^{-2}=\frac{m_{e}+R \sigma_{g}^{2}}{\nu_{g}^{\prime} n_{g}^{\prime}}\right)}{\operatorname{Pr}\left\{\chi_{\nu_{g}^{\prime}}^{2}<\frac{\nu_{g}^{\prime} m_{g}^{\prime}}{m_{e}}\right\}} I_{(0, \infty)}\left(\sigma_{g}^{2}\right)
$$

onde $\nu_{g}^{\prime}$ e $m_{g}^{\prime}$ serão obtidos pelas expressōes (145).

3.4.3.3 Distribuição Marginal a Posteriori Aproximada de $\sigma_{b}^{2}$

$$
p\left(\sigma_{b}^{2} \mid y\right) \doteq \frac{K R\left(\nu_{b} m_{b}\right)^{-1} p\left(\chi_{\nu_{b}}^{-2}=\frac{m_{g}{ }^{\prime \prime}+K R \sigma_{b}^{2}}{\nu_{b} m_{b}}\right)}{\operatorname{Pr}\left\{\chi_{\nu_{b}}^{2}<\frac{\nu_{b} m_{b}}{m_{g^{\prime \prime}}}\right\}} I_{(0, \infty)}\left(\sigma_{b}^{2}\right)
$$

onde $m_{g} "$ será calculado empregando-se a expressão (164). 
Utilizando-se os software's MATHEMATICA ou MAPLE V, probabilidades e algumas características de interesse, das distribuições marginais dos componentes da variância, poderão ser numericamente determinadas, por exemplo,

$$
\begin{gathered}
E\left[\sigma_{c}^{2} \mid \boldsymbol{y}\right] \doteq \int_{0}^{\infty} \sigma_{e}^{2} p\left(\sigma_{e}^{2} \mid \boldsymbol{y}\right) d \sigma_{e}^{2}, \quad \operatorname{Var}\left[\sigma_{e}^{2} \mid \boldsymbol{y}\right] \doteq \int_{0}^{\infty}\left(\sigma_{e}^{2}\right)^{2} p\left(\sigma_{e}^{2} \mid \boldsymbol{y}\right) d \sigma_{e}^{2}-E^{2}\left[\sigma_{e}^{2} \mid \boldsymbol{y}\right] \\
M o\left[\sigma_{e}^{2} \mid \boldsymbol{y}\right] \doteq \max \left\{p\left(\sigma_{e}^{2} \mid \boldsymbol{y}\right)\right\}, \quad \int_{0}^{M d\left[\sigma_{e}^{2} \mid \boldsymbol{y}\right]} p\left(\sigma_{e}^{2} \mid \boldsymbol{y}\right) d \sigma_{e}^{2}=0.5, \quad \text { etc. }
\end{gathered}
$$




\section{EXEMPLO ILUSTRATIVO}

Para ilustrar a metodologia desenvolvida, considerou-se um conjunto de dados experimentais referentes a um estudo sobre adubação em cana-deaçúcar.

\subsection{A Origem dos Dados e Hipóteses de Interesse}

Num estudo de adubação com micronutrientes em cana-de-açúcar, conduzido pela seção de Solos e Adubação da Coordenadoria Regional Norte do Programa Nacional de Melhoramento de Cana-de-açúcar - PLANALSUCAR, safra: 1982/1983, foram instalados très ensaios em usinas da Zona Litoral Norte do Estado de Pernambuco, com os seguintes tratamentos:

TRAT.1: Testemunha Absoluta

TRAT.2: NPK

TRAT.3: NPK + Micronutrientes + 0,0kg/ha de Cobre;

TRAT.4: NPK + Micronutrientes + $2,5 \mathrm{~kg} /$ ha de Cobre;

TRAT.5: NPK + Micronutrientes + 5,0kg/ha de Cobre;

TRAT.6: NPK + Micronutrientes + 7,5kg/ha de Cobre;

TRAT.7: NPK + Micronutrientes + 10,0kg/ha de Cobre.

OBS.: As quantidades de NPK e Micronutrientes aplicadas nos tratamentos de cada ensaio, foram as mesmas e de acordo com recomendação técnica.

Para efeito de análises estatísticas admitiu-se que interessa ao pesquisador a verificaçào das seguintes hipóteses: 
(a) - Os efeitos médios dos tratamentos são todos iguais, isto é,

$$
H_{0}^{(1)}: \theta_{1}=\theta_{2}=\ldots=\theta_{7}=\theta
$$

(b) - A diferença entre a produtividade média de açúcar do tratamento não adubado e a produtividade média dos tratamentos que foram adubados é nula, isto é,

$$
H_{0}^{(2)}: \phi_{1}=-\theta_{1}+\frac{\theta_{2}+\ldots+\theta_{7}}{6}=0
$$

(c) - A diferença entre a produtividade média de açúcar do tratamento adubado apenas com NPK e a produtividade média dos tratamentos que foram adubados com micronutrientes é nula, isto é,

$$
H_{0}^{(3)}: \phi_{2}=-\theta_{2}+\frac{\theta_{3}+\ldots+\theta_{7}}{5}=0
$$

(d) - Os componentes da regressão polinomial de primeiro a quarto graus associados ao comportamento da produtividade média de açúcar em relação aos níveis de Cobre na presença de NPK e Micronutrientes são todos nulos, isto é,

$$
\begin{aligned}
& H_{0}^{(4)}: \phi_{3}=-2 \theta_{3}-1 \theta_{4}+0 \theta_{5}+1 \theta_{6}+2 \theta_{7}=0 \quad \text { (componente de } 1 \text { o grau); } \\
& H_{0}^{(5)}: \phi_{4}=+2 \theta_{3}-1 \theta_{4}-2 \theta_{5}-1 \theta_{6}+2 \theta_{7}=0 \quad\left(\text { componente de } 2^{\circ} \text { grau }\right) ; \\
& H_{0}^{(6)}: \phi_{5}=-1 \theta_{3}+2 \theta_{4}+0 \theta_{5}-2 \theta_{6}+1 \theta_{7}=0 \quad \text { (componente de } 3^{\circ} \text { grau); } \\
& H_{0}^{(7)}: \phi_{6}=+1 \theta_{3}-4 \theta_{4}+6 \theta_{5}-4 \theta_{6}+1 \theta_{7}=0 \quad \text { (componente de } 4^{\circ} \text { grau); }
\end{aligned}
$$

(e) - O efeito do NPK sobre a produtividade média de açúcar é nula, isto é,

$$
H_{0}^{(8)}: \phi_{7}=\theta_{2}-\theta_{1}=0
$$

(f) - O efeito dos micronutrientes sobre a produtividade média de açúcar, na presença de NPK é nulo, isto é,

$$
H_{0}^{(9)}: \phi_{8}=\theta_{3}-\theta_{2}=0
$$


Os contrastes associados às hipóteses $H_{0}^{(2)}$ a $H_{0}^{(7)}$ são ortogonais. Este nào é o único conjunto de contrastes com esta característica. Existem vários outros. Entretanto, neste trabalho, admitiu-se que este conjunto é o de interesse do pesquisador.

Além das hipóteses citadas, interessa ao pesquisador informações acerca dos componentes da variância devidas ao fator experimento e interação, na análise conjunta, e ainda, sobre o erro experimental, nas análises individuais e conjunta.

\subsection{Os Dados Experimentais}

Os experimentos fora colhidos na safra $82 / 83$ e os resultados em toneladas de açúcar provável por hectare (TPH) encontram-se nas tabelas 3 a 5 , a seguir

Tabela 3: Valores de TPH observados no experimentais E1, instalado na Usina N. S. Maravilhas - Safra: 1982/1983.

\begin{tabular}{|l|rrrr||r|r|r|}
\hline Tratamento & Rep.1 & Rep.2 & Rep.3 & Rep.4 & Total & Média & $s_{i}^{2}$ \\
\hline \hline TRAT.1 & 7,03 & 7,29 & 4,99 & 4,72 & 24,03 & 6,01 & 1,7944 \\
\hline TRAT.2 & 8,42 & 5,39 & 7,03 & 7,40 & 28,24 & 7,06 & 1,5850 \\
\hline TRAT.3 & 5,38 & 6,17 & 7,14 & 7,40 & 26,09 & 6,52 & 0,8603 \\
\hline TRAT.4 & 6,30 & 8,24 & 8,12 & 9,05 & 31,71 & 7,93 & 1,3478 \\
\hline TRAT.5 & 9,50 & 8,23 & 10,83 & 8,34 & 36,90 & 9,22 & 1,4750 \\
\hline TRAT.6 & 9,65 & 10,08 & 8,17 & 9,51 & 37,41 & 9,35 & 0,6803 \\
\hline TRAT.7 & 11,16 & 9,90 & 11,27 & 8,58 & 40,91 & 10,23 & 1,5926 \\
\hline \hline - & - & - & - & 225,29 & 8,05 & 1,3336 \\
\hline \hline
\end{tabular}

Fonte: IAA - PLANALSUCAR - CONOR. 
Tabela 4: Valores de TPH observados no experimento E2, instalado na Usina Santa Tereza - Safra: 1982/1983.

\begin{tabular}{|l||rrrr||r|r|r|}
\hline \hline Tratamento & Rep.1 & Rep.2 & Rep.3 & Rep.4 & Total & Média & $s_{i}^{2}$ \\
\hline \hline TRAT.1 & 2,25 & 3,92 & 6,02 & 2,37 & 14,56 & 3,64 & 3,0959 \\
\hline TRAT.2 & 7,99 & 5,56 & 4,43 & 5,69 & 23,67 & 5,92 & 2,2292 \\
\hline TRAT.3 & 6,26 & 9,34 & 5,53 & 6,26 & 27,39 & 6,85 & 2,8796 \\
\hline TRAT.4 & 8,68 & 5,51 & 8,77 & 6,65 & 29,61 & 7,40 & 2,5500 \\
\hline TRAT.5 & 7,41 & 8,25 & 7,78 & 10,74 & 34,18 & 8,54 & 2,2595 \\
\hline TRAT.6 & 8,09 & 9,29 & 8,59 & 10,44 & 36,41 & 9,10 & 1,0373 \\
\hline TRAT.7 & 12,12 & 8,71 & 7,80 & 10,22 & 38,85 & 9,71 & 3,5721 \\
\hline \hline- & - & - & - & 204,67 & 7,31 & 2,5176 \\
\hline \hline
\end{tabular}

Fonte: IAA - PLANALSUCAR - CONOR.

Tabela 5: Valores de TPH observados no experimento E3, instalado na Usina São José - Safra: 1982/1983.

\begin{tabular}{|l||rrrr||r|r|r|}
\hline \hline Tratamento & Rep.1 & Rep.2 & Rep.3 & Rep.4 & Total & Média & $s_{i}^{2}$ \\
\hline \hline TRAT.1 & 4,57 & 5,03 & 3,69 & 4,23 & 17,52 & 4,38 & 0,3191 \\
\hline TRAT.2 & 7,23 & 8,05 & 7,01 & 6,73 & 29,02 & 7,25 & 0,3228 \\
\hline TRAT.3 & 7,78 & 8,64 & 6,90 & 6,59 & 29,91 & 7,48 & 0,8547 \\
\hline TRAT.4 & 8,80 & 7,26 & 7,55 & 6,96 & 30,57 & 7,64 & 0,6535 \\
\hline TRAT.5 & 9,96 & 8,49 & 8,86 & 8,23 & 35,54 & 8,88 & 0,5804 \\
\hline TRAT.6 & 9,22 & 9,30 & 9,91 & 8,61 & 37,04 & 9,26 & 0,2827 \\
\hline TRAT.7 & 9,64 & 10,57 & 8,41 & 9,74 & 38,36 & 9,59 & 0,7926 \\
\hline \hline- & - & - & - & - & 217,96 & 7,78 & 0,5437 \\
\hline
\end{tabular}

Fonte: IAA - PLANALSUCAR - CONOR. 


\subsection{As Análises Individuais}

\subsubsection{A Homogeneidade das Variâncias Dentro dos Ensaios}

O modelo proposto para as observações de cada ensaio, dado em (11), pressupõe além da normalidade e independência dos dados, que as variâncias dentro de cada tratamento em cada ensaio, seja a mesma, isto é, $\sigma_{1}^{2}=\sigma_{2}^{2}=\cdots=$ $\sigma_{7}^{2}$. Para verificar a ocorrência deste fato, foram aplicados o teste de Bartlett Modificado e o teste da Raiz Cúbica, conforme expressões dadas em (56) e (57), respectivamente. Para facilitar os cálculos, foram usadas tabelas auxiliares para fornecimento de resultados parciais, conforme se segue,

Tabela 6: Memória de cálculo para obtenção de resultados parciais a serem usados na aplicação dos testes de Bartlett Modificado e da Raiz Cúbica para os dados do experimento E1, isntalado na Usina N. S. Maravilhas

\begin{tabular}{ccccccccc}
\hline \hline$i$ & $\nu_{i}$ & $s_{i}^{2}$ & $a_{i}^{2}$ & $m_{i}$ & $\frac{1}{a_{i}^{2}}$ & $\frac{m_{i}}{a_{i}^{2}}$ & $\nu_{i}\left(\log s_{i}^{2}-\log \bar{s}^{2}\right)$ & $\frac{\left(m_{i}-m\right)^{2}}{a_{i}^{2}}$ \\
\hline \hline 1 & 3 & 1,7944 & 0,4733 & 1,0989 & 2,1128 & 2,3218 & 0,8904 & 0,0672 \\
2 & 3 & 1,5850 & 0,4357 & 1,1454 & 2,2952 & 2,6289 & 0,5181 & 0,0399 \\
3 & 3 & 0,8603 & 0,2899 & 1,4041 & 3,4495 & 4,8434 & $-1,3151$ & 0,0555 \\
4 & 3 & 1,3478 & 0,3911 & 1,2089 & 2,5569 & 3,0910 & 0,0318 & 0,0119 \\
5 & 3 & 1,4750 & 0,4153 & 1,1732 & 2,4079 & 2,8249 & 0,3023 & 0,0260 \\
6 & 3 & 0,6803 & 0,2479 & 1,5184 & 4,0339 & 6,1251 & $-2,0193$ & 0,2347 \\
7 & 3 & 1,5926 & 0,4371 & 1,1435 & 2,2878 & 2,6161 & 0,5325 & 0,0409 \\
\hline & 21 & & & & 19,1440 & 24,4512 & $-1,0593$ & 0,4761 \\
\hline \hline
\end{tabular}

De acordo com as expressões dadas em (58) e os resultados parciais da tabela 6, encontrou-se

$$
\begin{aligned}
& \nu_{i}=3, \quad \nu=21, \quad A=\frac{1}{3 \times 6}\left(\frac{7}{3}-\frac{1}{21}\right)=0,127, \\
& -2 \log W_{0}=1,0593, \quad m=\frac{24,4512}{19,1440}=1,2777,
\end{aligned}
$$




$$
\chi_{B M}^{2}=\frac{-2 \log W_{0}}{1+A}=0,940 \quad e \quad \chi_{R C}^{2}=\sum_{i=1}^{7} \frac{\left(m_{i}-m\right)^{2}}{a_{i}^{2}}=0,476 .
$$

Conforme foi visto, verificar a hipótese $H_{0}: \sigma_{1}^{2}=\sigma_{2}^{2}=\cdots=$ $\sigma_{7}^{2}=\sigma^{2}$, (para algum $\sigma^{2}$ desconhecido), a um nível de significância $\alpha=0,05$, é equivalente a estudar a densidade a posteriori de $\varphi=\left(\varphi_{1}, \varphi_{2}, \cdots, \varphi_{7}\right)^{\prime}$, onde $\varphi_{i}=2\left(\log \sigma_{i}-\log \sigma_{7}\right), i=1,2, \ldots, 6$, e verificar se o ponto particular $\varphi_{0}=\mathbf{0}\left(\varphi_{10}=\right.$ $\left.0, \varphi_{20}=0, \cdots, \varphi_{70}=0\right)^{\prime}$, está dentro ou fora da região H.P.D. que contém $95 \%$ de probabilidade. Neste sentido, o ponto $\varphi_{0}=0$ estará incluído na região H.P.D. que contém $95 \%$ de probabilidade se, e somente se,

$$
\begin{gathered}
\left.\chi_{B M}^{2}<\chi_{(6,0,05)}^{2} \quad \text { (Teste de Bartlett Modificado, }\right) \\
\chi_{R C}^{2}<\chi_{(6,0,05)}^{2} \quad \text { (Teste da Raiz Cúbica). }
\end{gathered}
$$

Ora, com 6 graus de liberdade e $\alpha=0,05$, a tabela da distribuição de qui-quadrado fornece $\chi_{(6,0,05)}^{2}=12,592$. Assim sendo, o ponto $\varphi_{0}=0$ está incluído na região H.P.D. Portanto, as variâncias podem ser consideradas homogêneas pelos dois testes.

Considerando-se os resultados da tabela 7 e as expressões dadas em (58), encontraram-se para o segundo experimento os seguintes resultados:

$$
\begin{gathered}
\nu_{i}=3, \quad \nu=21, \quad A=0,127, \quad-2 \log W_{0}=1,2387, \\
\chi_{B M}^{2}=\frac{-2 \log W_{0}}{1+A}=1,099 \quad \text { e } \quad \chi_{R C}^{2}=\sum_{i=1}^{7} \frac{\left(m_{i}-m\right)^{2}}{a_{i}^{2}}=0,320 . \\
\text { Como } \chi_{(6,0,05)}^{2}>\chi_{B M}^{2} \text { e } \chi_{(6,0,05)}^{2}>\chi_{R C}^{2}, \text { o ponto particular } \varphi_{0}=0
\end{gathered}
$$

está incluído na região H.P.D. e, portanto, as variâncias também são homogêneas dentro do segundo ensaio de acordo com os dois testes.

Analogamente, considerando-se os resultados da tabela 8 e as expressões dadas em (58), obteve-se,

$$
\begin{gathered}
\nu_{i}=3, \quad \nu=21, \quad A=0,127, \quad-2 \log W_{0}=1,8895 \\
\chi_{B M}^{2}=\frac{-2 \log W_{0}}{1+A}=1,677 \quad \text { e } \quad \chi_{R C}^{2}=\sum_{i=1}^{7} \frac{\left(m_{i}-m\right)^{2}}{a_{i}^{2}}=2,136 .
\end{gathered}
$$


Tabela 7: Memória de cálculo para obtenção de resultados parciais a serem usados na aplicação dos testes de Bartlett Modificado e da Raiz Cúbica para os dados do experimento E2, instalado na Usina Santa Tereza.

\begin{tabular}{ccccccccc}
\hline \hline$i$ & $\nu_{i}$ & $s_{i}^{2}$ & $a_{i}^{2}$ & $m_{i}$ & $\frac{1}{a_{i}^{2}}$ & $\frac{m_{i}}{a_{i}^{2}}$ & $\nu_{i}\left(\log s_{i}^{2}-\log \bar{s}^{2}\right)$ & $\frac{\left(m_{i}-m\right)^{2}}{a_{i}^{2}}$ \\
\hline \hline 1 & 3 & 3,0958 & 0,6808 & 0,9163 & 1,4689 & 1,3459 & 0,6202 & 0,0258 \\
2 & 3 & 2,2290 & 0,5469 & 1,0223 & 1,8285 & 1,8693 & $-0,3653$ & 0,0013 \\
3 & 3 & 2,8795 & 0,6487 & 0,9387 & 1,5415 & 1,4470 & 0,4029 & 0,0187 \\
4 & 3 & 2,5501 & 0,5982 & 0,9775 & 1,6717 & 1,6341 & 0,0385 & 0,0085 \\
5 & 3 & 2,2596 & 0,5519 & 1,0177 & 1,8119 & 1,8440 & $-0,3244$ & 0,0018 \\
6 & 3 & 1,0373 & 0,3284 & 1,3192 & 3,0451 & 4,0171 & $-2,6601$ & 0,2225 \\
7 & 3 & 3,5721 & 0,7489 & 0,8736 & 1,3353 & 1,1665 & 1,0495 & 0,0410 \\
\hline & 21 & & & & 12,7029 & 13,3239 & $-1,2387$ & 0,3196 \\
\hline \hline
\end{tabular}

Tabela 8: Memória de cálculo para obtenção de resultados parciais a serem usados na aplicação dos testes de Bartlett Modificado e da Raiz Cúbica para os dados do experimento E3, instalado na Usina São José.

\begin{tabular}{ccccccccc}
\hline \hline$i$ & $\nu_{i}$ & $s_{i}^{2}$ & $a_{i}^{2}$ & $m_{i}$ & $\frac{1}{a_{i}^{2}}$ & $\frac{m_{i}}{a_{i}^{2}}$ & $\nu_{i}\left(\log s_{i}^{2}-\log \bar{s}^{2}\right)$ & $\frac{\left(m_{i}-m\right)^{2}}{a_{i}^{2}}$ \\
\hline \hline 1 & 3 & 0,3191 & 0,1497 & 1,9542 & 6,6800 & 13,0541 & $-1,5987$ & 0,2162 \\
2 & 3 & 0,3227 & 0,1508 & 1,9469 & 6,6313 & 12,9105 & $-1,5650$ & 0,1976 \\
3 & 3 & 0,8547 & 0,2886 & 1,4072 & 3,4650 & 4,8760 & 1,3571 & 0,4670 \\
4 & 3 & 0,6535 & 0,2414 & 1,5389 & 4,1425 & 6,3749 & 0,5518 & 0,2295 \\
5 & 3 & 0,5805 & 0,2230 & 1,6009 & 4,4843 & 7,1789 & 0,1965 & 0,1348 \\
6 & 3 & 0,2827 & 0,1381 & 2,0347 & 7,2411 & 14,7335 & $-1,9620$ & 0,4910 \\
7 & 3 & 0,7926 & 0,2745 & 1,4430 & 3,6430 & 5,2568 & 1,1308 & 0,3999 \\
\hline & 21 & & & & & & & \\
\hline \hline
\end{tabular}


Como nos dois casos anteriores, $\chi_{(6,0,05)}^{2}>\chi_{B M}^{2}$ e $\chi_{(6,0,05)}^{2}>\chi_{R C}^{2}$, o ponto particular $\varphi_{0}=0$ está incluído na região H.P.D. e, portanto as variâncias também podem ser consideradas homogêneas dentro do terceiro experimento.

\subsubsection{As Distribuições Marginais a Posteriori de $\sigma^{2}, p\left(\sigma^{2} \mid \boldsymbol{y}\right)$, Relativas a Cada Experimento}

Os resultados obtidos na seção anterior, sugerem que é razoável admitir uma variância residual única para cada experimento, a qual é uma condição necessária para realização das análises da variância. Portanto, neste estágio, é conveniente apresentar as análises da variância individuais, cujos resultados encontram-se sintetizados na tabela 9 .

Tabela 9: Resultados das análises da variância dos três experimento considerados.

\begin{tabular}{|c|l|r|r|r|r|}
\hline \hline Experimento & F. Variação & GL & SQ & QM & F \\
\hline \hline E1 & Tratamento & 6 & 61,2748 & 10,2125 & 7,66 \\
& Resíduo & $\nu_{1}=21$ & $\nu_{1} s_{1}^{2}=28,0063$ & $s_{1}^{2}=1,3336$ & \\
\hline E2 & Tratamento & 6 & 104,5629 & 17,4271 & 6,924 \\
& Resíduo & $\nu_{2}=21$ & $\nu_{2} s_{2}^{2}=52,8705$ & $s_{2}^{2}=2,5176$ & \\
\hline \multirow{2}{*}{ E3 } & Tratamento & 6 & 74,5337 & 12,4223 & 22,85 \\
& Resíduo & $\nu_{3}=21$ & $\nu_{3} s_{3}^{2}=11,4174$ & $s_{3}^{2}=0,5437$ & \\
\hline
\end{tabular}

A tabela 9 fornece os elementos necessários à especificação das densidades a posteriori de cada ensaio. Ou seja, dadas as suposições e os dados, toda informação acerca do erro experimental de cada ensaio, considerando-se uma priori não informativa de referência, $p\left(\boldsymbol{\theta}, \sigma^{2}\right) \propto 1 / \sigma^{2}$, está contida na distribuição $a$ posteriori de $\sigma^{2}, p\left(\sigma^{2} \mid \boldsymbol{y}\right)$, dada pela expressão (26). Desta forma, as densidades a posteriori da variância residual encontradas para cada experimento, foram: 
(a) - Usina N. S. Maravilhas (E1)

$$
p\left(\sigma_{1}^{2} \mid \boldsymbol{y}\right)=\frac{(28,0063)^{10,5}}{\Gamma(10,5) 2^{10,5}}\left(\sigma_{1}^{2}\right)^{-11,5} e^{-\frac{28.0065}{2 \sigma_{1}^{2}}} I_{(0, \infty)}\left(\sigma_{1}^{2}\right),
$$

com as caracteísticas: $E\left(\sigma_{1}^{2} \mid \boldsymbol{y}\right)=1,4740, \operatorname{Var}\left(\sigma_{1}^{2} \mid \boldsymbol{y}\right)=0,2556, M o\left(\sigma_{1} \mid \boldsymbol{y}\right)=1,21767$, $M d\left(\sigma_{1}^{2} \mid \boldsymbol{y}\right)=1,37718$ e usando a expressão dada em (29), encontrou-se

$$
\operatorname{Pr}\left\{0,7668<\sigma_{1}^{2} \mid \boldsymbol{y}<2,6282\right\}=0,95
$$

(b) - Usina Santa Tereza (E2)

$$
p\left(\sigma_{2}^{2} \mid \boldsymbol{y}\right)=\frac{(52,8705)^{10,5}}{\Gamma(10,5) 2^{10,5}}\left(\sigma_{1}^{2}\right)^{-11,5} e^{-\frac{52,8705}{2 \sigma_{2}^{2}}} I_{(0, \infty)}\left(\sigma_{2}^{2}\right),
$$

com as características: $E\left(\sigma_{2}^{2} \mid \boldsymbol{y}\right)=2,7827, \operatorname{Var}\left(\sigma_{2}^{2} \mid \boldsymbol{y}\right)=0,9110, M o\left(\sigma_{2}^{2} \mid \boldsymbol{y}\right)=$ 2, 51764, $M d\left(\sigma_{2}^{2} \mid \boldsymbol{y}\right)=2,5997$ e usando a expressão dada em (29), encontrou-se

$$
\operatorname{Pr}\left\{1,4475<\sigma_{2}^{2} \mid \boldsymbol{y}<4,9616\right\}=0,95
$$

(c) - Usina São José (E3)

$$
p\left(\sigma_{3}^{2} \mid \boldsymbol{y}\right)=\frac{(11,4174)^{10,5}}{\Gamma(10,5) 2^{10,5}}\left(\sigma_{1}^{2}\right)^{-11,5} e^{-\frac{11,4174}{2 \sigma_{3}^{2}}} I_{(0, \infty)}\left(\sigma_{3}^{2}\right),
$$

e tem as características: $E\left(\sigma_{3}^{2} \mid \boldsymbol{y}\right)=0,6009, \operatorname{Var}\left(\sigma_{3}^{2} \mid \boldsymbol{y}\right)=0,0425, \operatorname{Mo}\left(\sigma_{2}^{2} \mid \boldsymbol{y}\right)=$ $0,543686, M d\left(\sigma_{2}^{2} \mid \boldsymbol{y}\right)=0,56142$ e usando a expressão dada em (29), encontrou-se

$$
\operatorname{Pr}\left\{0,3126<\sigma_{3}^{2} \mid \boldsymbol{y}<1,0715\right\}=0,95 .
$$

A figura 1 apresenta os gráficos (a), (b) e (c) referentes às densidades posteriori do erro experimental de cada ensaio, bem como, o gráfico conjunto (d) para possibilitar a comparação do comportamento probabilístico dos três erros experimentais. Neste sentido, observa-se que o erro experimental do ensaio (c), é bem menos disperso que os outros dois. Este fato pode ser explicado pelas condições (solo homogêneo, controle experimental, material experimental, etc.) do ensaio (c) terem sido melhores que as dos demais ensaios. 
Figura 1: Gráficos das densidades a posteriori do erro experimental de cada ensaio (a), (b), (c) e gráfico conjunto (d).

p1 1

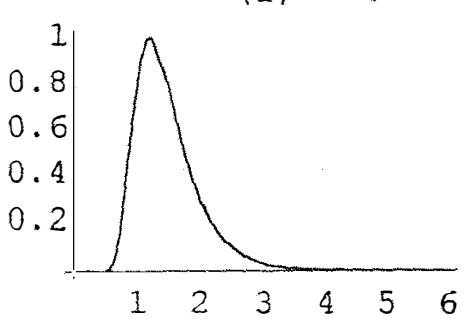

p3

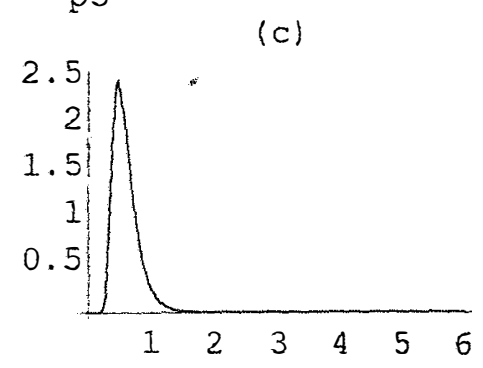

p2

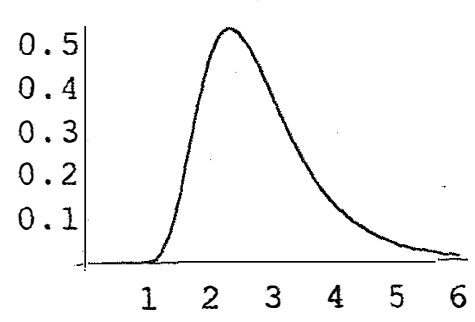

$p$

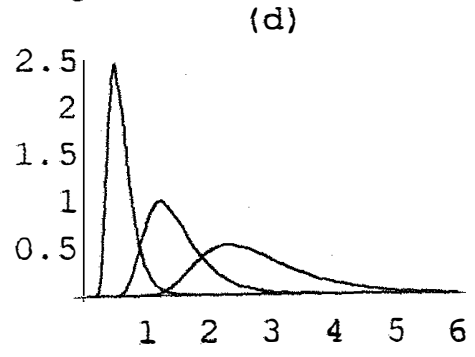

4.3.3 Distribuição Conjunta a Posteriori para os Efeitos Médios dos Tratamentos, $p(\theta \mid y)$, em Cada Experimento

Dadas as suposições e os dados experimentais, toda informação relativa ao vetor de parâmetros $\boldsymbol{\theta}=\left(\theta_{1}, \theta_{2}, \cdots, \theta_{7}\right)^{\prime}$, sob a luz de uma priori nãoinformativa de referência, $p\left(\theta, \sigma^{2}\right) \propto 1 / \sigma^{2}$, estará contida na distribuição a posteriori de $\boldsymbol{\theta}, p(\boldsymbol{\theta} \mid \boldsymbol{y})$, a qual por $(31)$, é uma t-Student multivariada de dimensão 7 , isto é,

$$
\boldsymbol{\theta}\left\{\boldsymbol{y} \sim t_{7}\left\{\hat{\boldsymbol{\theta}} ; s^{2}\left(X^{\prime} X\right)^{-1} ; \nu\right\}\right.
$$

onde $\hat{\boldsymbol{\theta}}, s^{2}$ e $\nu$ são, respectivamente, o vetor das médias dos tratamentos, o quadrado médio do resíduo e os graus de liberdade associados aos resíduos de cada ensaio. Assim, para o experimento da Usina São José (E3), por exemplo, tem-se que, 


$$
\boldsymbol{\theta} \mid \boldsymbol{y} \sim t_{7}\left(\left[\begin{array}{c}
4,38 \\
7,25 \\
7,48 \\
7,64 \\
8,88 \\
9,26 \\
9,59
\end{array}\right] ;\left[\begin{array}{ccccccc}
1 & 0 & 0 & 0 & 0 & 0 & 0 \\
0 & 1 & 0 & 0 & 0 & 0 & 0 \\
0 & 0 & 1 & 0 & 0 & 0 & 0 \\
0 & 0 & 0 & 1 & 0 & 0 & 0 \\
0 & 0 & 0 & 0 & 1 & 0 & 0 \\
0 & 0 & 0 & 0 & 0 & 1 & 0 \\
0 & 0 & 0 & 0 & 0 & 0 & 1
\end{array}\right] \text { } ; 21\right)
$$

Supondo que, neste trabalho, há interesse em verificar se o ponto particular $\theta_{0}=\left(\theta_{10}=4, \theta_{20}=5, \theta_{30}=6, \theta_{40}=7, \theta_{50}=8, \theta_{60}=9, \theta_{70}=10\right)^{\prime}$ está dentro da região H.P.D. que contém $95 \%$ de probabilidade. Isto por (48) ocorrerá se. e somente se

$$
\frac{Q\left(\boldsymbol{\theta}_{0}\right)}{K s^{2}}=\frac{\left(\boldsymbol{\theta}_{0}-\hat{\boldsymbol{\theta}}\right)^{\prime}\left(X^{\prime} X\right)\left(\boldsymbol{\theta}_{0}-\hat{\boldsymbol{\theta}}\right)}{7 \times 0,5437}=\frac{4 \sum_{i=1}^{\tau}\left(\boldsymbol{\theta}_{0}-\hat{\boldsymbol{\theta}}\right)^{2}}{3,8059}<F_{[\tau, 21,0,05]}
$$

onde

$$
\left(\boldsymbol{\theta}_{0}-\hat{\boldsymbol{\theta}}\right)=\left[\begin{array}{r}
4-4,38 \\
5-7,25 \\
6-7,48 \\
7-7,64 \\
8-8,88 \\
9-9,26 \\
10-9,59
\end{array}\right]=\left[\begin{array}{r}
-0,38 \\
-2,25 \\
-1,48 \\
-0,64 \\
-0,88 \\
-0,26 \\
0,41
\end{array}\right] \Longrightarrow \frac{4 \sum_{i=1}^{7}\left(\boldsymbol{\theta}_{0}-\hat{\boldsymbol{\theta}}\right)^{2}}{3,8059}=9,27
$$

Como $\frac{Q\left(\boldsymbol{\theta}_{0}\right)}{K^{\prime} \boldsymbol{s}^{2}}=9,27$ não é menor que $F_{[\tau, 21,0,05]}=2,99$, o ponto $\boldsymbol{\theta}_{0}$ está excluído da regiào H.P.D. Isto é equivalente a rejeitar a hipótese $H_{0}: \theta=\boldsymbol{\theta}_{0}$ a um nível de significància $\alpha=0,05$,

\subsubsection{Distribuição Marginal a Posteriori de $\theta_{i}, p\left(\theta_{i} \mid y\right)$}

De acordo com (37), a distribuição marginal a posteriori de $\theta_{i}$, $i=1,2, \cdots, 7$, em cada experimento $j, j=1,2,3$, é t-Student univariada com 
média $\hat{\theta}_{i}$ variância $\frac{s_{j}^{2}}{R}$ e $\nu=21$ graus de liberdade. Isto é, $\theta_{i} \mid \boldsymbol{y} \sim t_{1}\left(\hat{\theta}_{i}, \frac{s_{j}^{2}}{R}, \nu\right)$. Assim, por exemplo, para o ensaio da Usina São José, $\theta_{6}$ é distribuído a posteriori como,

$$
\theta_{6} \mid \boldsymbol{y} \sim t_{1}\left(9,26, \frac{0,5437}{4}, 21\right) .
$$

cuja função de densidade a posteriori é dada por

$$
p\left(\theta_{6} \mid \boldsymbol{y}\right)=\frac{\Gamma(11) 4^{\frac{1}{2}}(0,5437)^{-\frac{1}{2}}}{\Gamma(0,5) \Gamma(10,5) 21^{\frac{1}{2}}}\left[1+\frac{\left.4\left(\theta_{6}-9,26\right)^{2}\right]^{-11}}{11,4177} I_{(-\infty,+\infty)}\left(\theta_{6}\right)\right.
$$

Caso haja interesse em calcular alguma probabilidade acerca de $\theta_{6}$ do ensaio da Usina São José, a densidade acima deve ser utilizada.

Os intervalos posteriori com $95 \%$ de credibilidade, para cada $\theta_{i}$, $i=1,2, \ldots, 7$, em cada ensaio $j, j=1,2,3$, foram determinados de acordo com a expressão (38) e encontram-se apresentados na tabela 10.

Tabela 10: Intervalos com $95 \%$ de credibilidade para as médias dos tratamentos em cada experimento.

\begin{tabular}{|c|c|c|c|}
\hline \hline Parâmetro & E1 & E2 & E3 \\
\hline \hline$\theta_{1}$ & {$[4,81 ; 7,21]$} & {$[1,99 ; 5,29]$} & {$[3,61 ; 5,15]$} \\
$\theta_{2}$ & {$[5,86 ; 8,26]$} & {$[4,27 ; 7,57]$} & {$[6,48 ; 8,02]$} \\
$\theta_{3}$ & {$[5,32 ; 7,72]$} & {$[5,20 ; 8,50]$} & {$[6,51 ; 8,05]$} \\
$\theta_{4}$ & {$[6,73 ; 9,13]$} & {$[5,75 ; 9,05]$} & {$[6,87 ; 8,41]$} \\
$\theta_{5}$ & {$[8,02 ; 10,42]$} & {$[6,89 ; 10,19]$} & {$[8,11 ; 9,45]$} \\
$\theta_{6}$ & {$[8,15 ; 10,55]$} & {$[7,45 ; 10,75]$} & {$[8,49 ; 10,03]$} \\
$\theta_{7}$ & {$[9,03 ; 11,43]$} & {$[8,06 ; 11,36]$} & {$[8,82 ; 10,36]$} \\
\hline \hline
\end{tabular}

Sendo assim, o elemento correspondente a linha 1 coluna 2 da tabela 10, por exemplo, é interpretado como: a probabilidade a posteriori de $\theta_{1}$ (a média do tratamento 1 no ensaio da Usina N. S. Maravilhas (E1)) assumir um valor no intervalo $[4,81 ; 7,21]$ é de $95 \%$, o que é diferente da interpretação de um intervalo de confiança. 


\subsubsection{Comparações dos Efeitos Médios dos Tratamentos}

As comparações dos parâmetros foram feitas mediante a distribuição a posteriori conjunta de $\phi$, dada em (44) e levando-se em consideração as hipóteses formuladas no planejamento da pesquisa, as quais encontram-se relacionadas no início da seção 4.1, ítens de (a) a (f).

Verificar a hipótese $H_{0}^{(1)}: \boldsymbol{\phi}=\mathbf{0}\left(\theta_{1}=\theta_{2}=\cdots=\theta_{7}=\theta\right)^{\prime}$, para algum $\theta$ desconhecido, a um nível de significância $\alpha=0,05$, é equivalente a verificar se o ponto zero está dentro ou fora da região H.P.D. que contém $95 \%$ de probabilidade, onde $\phi=D \boldsymbol{\theta}$ é qualquer conjunto de 6 contrastes independentes dos $\theta_{i}$ 's. Neste sentido, por (50), $\boldsymbol{\phi}_{0}=\mathbf{0}$ estará incluído na região H.P.D. que contém $95 \%$ de probabilidade se, e somente se,

$$
\frac{Q\left(\phi_{0}\right)}{K-1}=\frac{\left(\phi_{0}-\hat{\phi}\right)^{\prime}\left[D\left(X^{\prime} X\right)^{-1} D^{\prime}\right]^{-1}\left(\phi_{0}-\hat{\phi}\right)}{6 s^{2}}<F_{[6 ; 21 ; 0,05]}
$$

onde a matriz $D$ pode ser, por exemplo, a matriz dos coeficientes dos contrastes associados às hipóteses $H_{0}^{(2)}$ a $H_{0}^{(7)}$. Assim, para o ensaio da Usina N. S. Maravilhas (E1), tem-se

$$
\begin{aligned}
\hat{\phi} & =D \hat{\boldsymbol{\theta}}= \\
& =\left[\begin{array}{c}
\hat{\phi}_{1} \\
\hat{\phi}_{2} \\
\hat{\phi}_{3} \\
\hat{\phi}_{4} \\
\hat{\phi}_{5} \\
\hat{\phi}_{6}
\end{array}\right]=\frac{1}{4}\left[\begin{array}{ccccccc}
-6 & 1 & 1 & 1 & 1 & 1 & 1 \\
0 & -5 & 1 & 1 & 1 & 1 & 1 \\
0 & 0 & -2 & -1 & 0 & 1 & 2 \\
0 & 0 & 2 & -1 & -2 & -1 & 2 \\
0 & 0 & -1 & 2 & 0 & -2 & 1 \\
0 & 0 & 1 & -4 & 6 & -4 & 1
\end{array}\right]\left[\begin{array}{r}
24,03 \\
28,24 \\
26,09 \\
31,71 \\
36,90 \\
37,41 \\
40,91
\end{array}\right]=\left[\begin{array}{r}
14,270 \\
7,955 \\
8,835 \\
-2,230 \\
0,855 \\
2,980
\end{array}\right] \\
H & =\left(h_{j j}\right)=\left[D\left(X^{\prime} X\right)^{-1} D^{\prime}\right]=\frac{1}{R}\left[\begin{array}{cccc}
\sum_{i=1}^{7} d_{1 i}^{2} & 0 & \ldots & 0 \\
0 & \sum_{i=1}^{7} d_{2 i}^{2} & \cdots & 0 \\
\vdots & \vdots & \ddots & \vdots \\
0 & 0 & \ldots & \sum_{i=1}^{7} d_{6 i}^{2}
\end{array}\right]
\end{aligned}
$$




$$
=\frac{1}{4}\left[\begin{array}{rrrrrr}
42 & 0 & 0 & 0 & 0 & 0 \\
0 & 30 & 0 & 0 & 0 & 0 \\
0 & 0 & 10 & 0 & 0 & 0 \\
0 & 0 & 0 & 14 & 0 & 0 \\
0 & 0 & 0 & 0 & 10 & 0 \\
0 & 0 & 0 & 0 & 0 & 70
\end{array}\right] e
$$

De modo análogo, encontrou-se para os ensaios da Usina Santa Tereza (E2) e Usina São.José (E3),

$$
\begin{gathered}
\dot{\phi}=\left[\begin{array}{r}
25,6875 \\
12,0225 \\
7,43000 \\
-0,4750 \\
-0,5350 \\
1,8100
\end{array}\right] \quad \epsilon \quad \dot{\phi}=\left[\begin{array}{r}
23,8300 \\
6,5800 \\
5,8425 \\
-0,5375 \\
-1.1225 \\
2,7675,
\end{array}\right]: \\
\frac{Q(0)}{6 \times 2,5176}=\frac{17,4271}{2,5176}=6,92 \quad \epsilon \quad \frac{Q(0)}{6 \times 0,5437}=\frac{12,4223}{0,5437}=22,85
\end{gathered}
$$

respectivamente.

Como as quantidades $\frac{Q(0)}{6 \times s^{2}}>F_{[6,21,0,05]}=2,99$ nos très ensaios, o ponto zero está excluído na região H.P.D. Isto equivale a rejeitar a hipótese $H_{0}^{(1)}$ : $\phi=0\left(\theta_{1}=\theta_{2}=\cdots=\theta_{7}=\theta\right)$ a um nível de significància $\alpha=0,05$.

\subsubsection{Distribuições Marginais a Posteriori dos Contrastes $\phi_{j}, p\left(\phi_{j} \mid \boldsymbol{y}\right)$}

A distribuição marginal a posteriori de $\phi_{j}=\sum_{i=1}^{7} d_{j i} \theta_{i}, \forall j, j=$ 1. 2. . . 8. com $\sum_{i=1}^{7} d_{j i}=0$, é t-Student univariada com média $\grave{\phi}_{j}$, variância $s^{2} h_{j j}$ e 21 graus de liberdade, isto é.

$$
\phi_{j} \mid y \sim t_{1}\left[\hat{\phi}_{j}, s^{2} h_{j j}, 21\right]
$$


A densidade a posteriori de $\phi_{1}=-6 \theta_{1}+\theta_{2}+\cdots+\theta_{7}$, encontrada para o ensaio da Usina N. S. Maravilhas (E1), por exemplo, foi conforme (45),

$$
p\left(\phi_{1} \mid \boldsymbol{y}\right)=\frac{\Gamma(11)|42 / 4|^{-\frac{1}{2}}(1,3336)^{-\frac{1}{2}}}{\Gamma(0,5) \Gamma(10,5) 21^{\frac{1}{2}}}\left[1+\frac{\left(\phi_{1}-14,27\right)^{2}}{21 \times 1,3336 \times \frac{42}{4}}\right]^{-11} I_{(-\infty,+\infty)}\left(\phi_{1}\right),
$$

que resulta em

$$
p\left(\phi_{1} \mid \boldsymbol{y}\right)=0,1053\left[1+\frac{\left(\phi_{1}-14,27\right)^{2}}{294,0588}\right]^{-11} I_{(-\infty,+\infty)}\left(\phi_{1}\right)
$$

de onde segue que

$$
\left.E\left(\phi_{1} \mid \boldsymbol{y}\right)\right]=\hat{\phi}_{1}=14,27 \quad e \quad \operatorname{Var}\left[\phi_{1} \mid \boldsymbol{y}\right]=s^{2} h_{11}=1,3336 \times \frac{42}{4}=14,0028
$$

Além disso, supondo que há interesse em saber a probabilidade a posteriori de que a média dos tratamentos adubados seja superior a média da testemunha, na Usina N. S. Maravilhas (E2), esta pode ser determinada a partir de $p\left(\phi_{1} \mid \boldsymbol{y}\right)$, ou seja,

$$
\operatorname{Pr}\left\{\frac{\theta_{2}+\cdots+\theta_{7}}{6}>\theta_{1}\right\}=\operatorname{Pr}\left\{-6 \theta_{1}+\theta_{2}+\cdots+\theta_{\mathbf{7}}>0\right\}=\operatorname{Pr}\left\{\phi_{1}>0\right\} .
$$

Segue que

$$
\operatorname{Pr}\left\{\phi_{1}>0\right\}=0,1053 \int_{0}^{\infty}\left[1+\frac{\left(\phi_{1}-14,24\right)^{2}}{294,0588}\right]^{-11} d \phi_{1}=0,999009
$$

Com auxílio dos Software's MATHEMATICA ou MAPLE V, o resultado acima é facilmente obtido, digitando-se apenas a linha Integrate[f, $\{\mathbf{x}, 0,1000\}]$ para o MATHEMATICA ou evalf(int $(\mathbf{f}, \mathbf{x}=\mathbf{0 . . 1 0 0 0 )})$ para o MAPLE $\mathbf{V}$, onde $f=$ $p\left(\dot{\phi}_{1} \mid \boldsymbol{y}\right)$ e $x=\phi_{1}$.

Os intervalos com $95 \%$ de credibilidade para os contrastes $\phi_{j}, j=$ $1,2, \cdots, 8$, obtidos a partir da expressão dada em (47), encontram-se na tabela 11.

O elemento correspondente ao contraste $\phi_{1}$ e ensaio $E 1$ da tabela 11, por exemplo, é interpretado como: a probabilidade marginal a posteriori de que o contraste $\phi_{1}$, na Usina N. S. Maravilhas (E1), assuma um valor no intervalo $(6,49,22,05)$ é de 0,95 . Além disso, verificar as hipóteses marginais $H_{0}^{(2)}: \phi_{1}=0$ 
Tabela 11: Intervalos com $95 \%$ de credibilidade para os contrastes entre as médias dos tratamentos, associados às hipóteses $H_{0}^{(2)}$ a $H_{0}^{(9)}$, para os três ensaios.

\begin{tabular}{|c|c|c|c|}
\hline \hline Contraste & E1 & E2 & E3 \\
\hline \hline$\phi_{1}$ & {$[6,49 ; 22,05]$} & {$[14,94 ; 36,38]$} & {$[18,86 ; 28,80]$} \\
$\phi_{2}$ & {$[1,38 ; 14,53]$} & {$[2,98 ; 21,06]$} & {$[2,38 ; 10,78]$} \\
$\phi_{3}$ & {$[5,04 ; 12,63]$} & {$[2,21 ; 12,65]$} & {$[3,42 ; 8,27]$} \\
$\phi_{4}$ & {$[-6,72 ; 2,26]$} & {$[-6,65 ; 5,70]$} & {$[-3,41 ; 2,33]$} \\
$\phi_{5}$ & {$[-2,94 ; 4,65]$} & {$[-5,75 ; 4,68]$} & {$[-3,55 ; 1,30]$} \\
$\phi_{6}$ & {$[-7,07 ; 13,03]$} & {$[-12,00 ; 15,62]$} & {$[-3,65 ; 9,18]$} \\
\hline$\phi_{7}$ & {$[-0,65 ; 2,75]$} & {$[-0,06 ; 4,61]$} & {$[1,79 ; 3,96]$} \\
$\phi_{8}$ & {$[-2,24 ; 1,16]$} & {$[-1,40 ; 3,26]$} & {$[-0,86 ; 1,31]$} \\
\hline \hline
\end{tabular}

a $H_{0}^{(9)}: \dot{\phi}_{8}=0$, a um nível de significància $\alpha=0,05$, é equivalente a verificar se o ponto zero está nos intervalos de credibilidade apresentados na tabela 11. Caso o zero esteja incluído aceita-se $H_{0}$ caso contrário rejeita-se. Por exemplo, o intervalo de credibilidade correspondente ao contraste $\phi_{3}$, não contém o ponto zero, enquanto que os intervalos de credibilidade correspondentes aos contrastes $\phi_{4}, \phi_{5}$ e $\phi_{6}$ incluem o ponto zero, em todos os ensaios. Isto indica que as variações da produtividade de açúcar em relação aos níveis de Cobre aplicados no solo, na presença de NPK e Micronutrientes, foi linear para todos os ensaios. O ponto zero não está incluído nos intervalos com $95 \%$ de credibilidade dos contrastes $\phi_{1}$ e $\phi_{2}$, indicando que estes são marginalmente diferentes de zero com mais de $95 \%$ de probabilidade a posteriori. Com exceção do contraste $\phi_{7}$ na Usina São José (E3), os intervalos H.P.D. para $\phi_{7}$ e $\dot{\phi}_{8}$ incluem o ponto zero.

É importante lembrar, mais uma vez, que as probabilidades conjuntas não podem ser obtidas a partir das densidades marginais, pois os intervalos marginais de alta densidade a posteriori que contém $100(1-\alpha) \%$ de probabilidade, podem incluir pontos que estarão excluídos da região H.P.D. conjunta ou vice-versa. 
Este é um problema bastante discutido no enfoque clássico, nos casos onde é importänte a preservação do nível de significância conjunto.

\subsection{A Análise Conjunta dos Experimentos}

Antes de proceder à análise conjunta, é conveniente verificar a homogeneidade das variâncias residuais entre os experimentos. Tal como nas análise individuais, aplicaram-se os testes de Bartlett Modificado e o da Raiz Cúbica. A tabela 12, fornece os resultados parciais necessários à aplicação dos mesmos.

Tabela 12: Memória de cálculo para obtenção de resultados parciais a serem usados na aplicação dos testes de Bartlett Modificado e da Raiz Cúbica para a análise conjunta dos três experimentos

\begin{tabular}{ccccccccc}
\hline \hline$i$ & $\nu_{i}$ & $s_{i}^{2}$ & $a_{i}^{2}$ & $m_{i}$ & $\frac{1}{a_{i}^{2}}$ & $\frac{m_{i}}{a_{i}^{2}}$ & $\nu_{i}\left(\log s_{i}^{2}-\log \bar{s}^{2}\right)$ & $\frac{\left(m_{i}-m\right)^{2}}{a_{i}^{2}}$ \\
\hline 1 & 21 & 1,3336 & 0,7428 & 2,4800 & 1,3463 & 3,3387 & $-1,9734$ & 0,1778 \\
2 & 21 & 2,5176 & 1,1346 & 2,0066 & 0,8814 & 1,7686 & 11,3705 & 0,6172 \\
3 & 21 & 0,5437 & 0,4084 & 3,3445 & 2,4486 & 8,1893 & $-20,8155$ & 0,6148 \\
\hline & 63 & & & & 4,6763 & 13,2966 & $-11,4184$ & 1,4100 \\
\hline \hline
\end{tabular}

Considerando-se os resultados da tabela 12 e as expressões dadas em (58), encontraram-se para a análise conjunta seguintes resultados:

$$
\begin{gathered}
\nu_{i}=21, \quad \nu=63, \quad A=\frac{1}{3 \times 2}\left(\frac{3}{21}-\frac{1}{63}\right)=0,0212 \\
-2 \log W_{0}=11,4184, \quad m=\frac{13,2966}{4,6763} \\
\chi_{B M}^{2}=\frac{-2 \log W_{0}}{1+A}=11,181 \quad \text { e } \chi_{R C}^{2}=\sum_{i=1}^{3} \frac{\left(m_{i}-m\right)^{2}}{a_{i}^{2}}=1,410 . \\
\text { Ora, } \chi_{(2 ; 0 ; 05)}^{2}=5,991 . \quad \text { Assim, } \chi_{(2 ; 0,05)}^{2}<\chi_{B M}^{2} \text { e } \chi_{(6,0,05)}^{2}>\chi_{R C}^{2} .
\end{gathered}
$$

Portanto, o teste de Bartlett mostru que as variâncias dos très experimentos são 
heterogèneas enquanto que o teste da Raiz Cúbica mostrou que as mesmas são homogèneas.

Admitindo que os erros são normais e independentes e que o teste da. Raiz Cúbica apresenta melhor desempenho neste caso, decidiu-se por aceitar a homogeneidade das variâncias entre os experimentos e proceder à análise conjunta. E. para este fim, é conveniente determinar preliminarmente algumas quantidades, as quais devem ser obtidas a partir dos elementos constantes da expressão (78). Assim, a tabela 13, apresenta os resultados obtidos a partir dos dados dos três ensaios considerados.

Tabela 13: Elementos necessárias para realização da análise conjunta Bayesiana, obtidas a partir dos dados experimentais.

\begin{tabular}{|l|r|r|r|}
\hline F. Variação & GL & \multicolumn{1}{|c|}{ SQ } & \multicolumn{1}{c|}{ QM } \\
\hline \hline Tratamento(T) & $\nu_{\phi}=6$ & $\nu_{\phi} m(\phi)=227,8570$ & $m(\phi)=37,9762$ \\
Ensaio(E) & $\nu_{b}=2$ & $\nu_{b} m_{b}=7,8040$ & $m_{b}=3,9020$ \\
Int. T $\times \mathrm{E}$ & $\nu_{g}=12$ & $\nu_{g} m_{g}=12,5143$ & $m_{g}=1,0429$ \\
Resíduo & $\nu_{e}=63$ & $\nu_{e} m_{e}=92,2941$ & $m_{e}=1,4650$ \\
\hline \hline
\end{tabular}

Dadas as suposições e os dados dos três ensaios, toda informação a respeito dos efeitos comparativos dos tratamentos, estará presente na distribuição a posteriori de $\phi, p(\phi \mid \boldsymbol{y})$, dada pela expressão (95), a qual é o produto de uma t-Student pelo fator modificador, $g(\phi)$. Devido à dificuldade de se trabalhar com a distribuição exata, na prática, as inferências sobre os efeitos comparativos dos tratamentos, são feitas a partir da distribuição a posteriori aproximada de $\phi$, a qual é uma t-Student multivariada de dimensão 6 , com média $\hat{\boldsymbol{\phi}}=D \hat{\boldsymbol{\theta}}$, matriz de variâncias e covariâncias $m_{g}^{*} \sum$ e $\nu_{g}^{*}$ graus de liberdade, denotada por $\phi \mid y \dot{\sim}$ $t_{6}\left[\dot{\phi}, m_{g}^{*} \sum, \nu_{g}^{*}\right]$, onde, de acordo com as hipóteses de interesse neste trabalho, os elementos da distribuição, $\hat{\phi}, \sum, \nu_{g}^{*}$ e $m_{g}^{*}$ foram obtidos como segue. 


$$
\begin{aligned}
& \hat{\phi}=D \hat{\boldsymbol{\theta}}= \\
& =\frac{1}{12}\left[\begin{array}{ccccccc}
-6 & 1 & 1 & 1 & 1 & 1 & 1 \\
0 & -5 & 1 & 1 & 1 & 1 & 1 \\
0 & 0 & -2 & -1 & 0 & 1 & 2 \\
0 & 0 & 2 & -1 & -2 & -1 & 2 \\
0 & 0 & -1 & 2 & 0 & -2 & 1 \\
0 & 0 & 1 & -4 & 6 & -4 & 1
\end{array}\right]\left[\begin{array}{r}
y_{1 . .}=56,11 \\
y_{2 .}=80,93 \\
y_{3 .}=83,39 \\
y_{4 . .}=91,89 \\
y_{5 .}=106,62 \\
y_{6 .}=110,86 \\
y_{7 .}=118,12
\end{array}\right]=\left[\begin{array}{r}
21,2625 \\
8,8525 \\
7,3692 \\
-1,0808 \\
-0,2675 \\
2,5192
\end{array}\right], \\
& \sum=\left[D\left(X^{\prime} X\right)^{-1} D^{\prime}\right]=\frac{1}{J R}\left[\begin{array}{cccc}
\sum_{i=1}^{K} d_{1 i}^{2} & 0 & \cdots & 0 \\
0 & \sum_{i=1}^{K} d_{2 i}^{2} & \cdots & 0 \\
\vdots & \vdots & \ddots & \vdots \\
0 & 0 & \cdots & \sum_{i=1}^{K} d_{6 i}^{2}
\end{array}\right]= \\
& =\frac{1}{12}\left[\begin{array}{cccccc}
42 & 0 & 0 & 0 & 0 & 0 \\
0 & 30 & 0 & 0 & 0 & 0 \\
0 & 0 & 10 & 0 & 0 & 0 \\
0 & 0 & 0 & 14 & 0 & 0 \\
0 & 0 & 0 & 0 & 10 & 0 \\
0 & 0 & 0 & 0 & 0 & 70
\end{array}\right], \quad \nu_{g}^{*} \doteq 27 \quad \text { e } \quad m_{g}^{*} \doteq 1,8880
\end{aligned}
$$

O quadrado médio da interação $m_{g}^{*}$, e os graus de liberdade $\nu_{g}^{*}$, foram obtidos como segue.

(a) Pelas expressões dadas em (106), tem-se que,

$$
\begin{gathered}
y=\frac{\nu_{b} m_{b}}{\nu_{b} m_{b}+\nu_{e} m_{e}}=0,3841, \quad P_{1}=I_{y}(1,7)=0,9664, \quad P_{2}=I_{y}(1,8)=0,9793, \\
P_{3}=I_{y}(1,6)=0,9454 \quad \text { onde } \quad I_{y}(p, q)=\frac{1}{B(p, q)} \int_{0}^{y} u^{p-1}(1-u)^{q-1} d u
\end{gathered}
$$

As integrais, denotadas por $I_{y}(p, q)$, podem ser calculadas facilmente com auxílio do software MATHEMATICA, por exemplo, digitando-se apenas a linha de comandos: NIntegrate $\left[(1 /(\operatorname{Beta}[\mathbf{p}, \mathbf{q}]))^{*}\left(\mathbf{u}^{\sim}(\mathbf{p}-1)\right)^{*}\left((\mathbf{1}-\mathbf{u})^{-}(\mathbf{q}-1)\right),\{u, 0, y\}\right]$; 
(b) Usando as expressões dadas em (107) e (108), vem

$$
\begin{array}{cc}
a=\frac{\nu_{g}+2}{2} \frac{P_{2}}{P_{1}}-\frac{\nu_{g} P_{1}}{2 P_{3}}=0,9602 . & b=\frac{\nu_{g}}{a} \frac{P_{1}}{P_{3}}=12,7750=\nu_{g}^{\prime}, \\
m_{g}^{\prime}=\frac{\nu_{g} m_{g}}{a \nu_{g}^{\prime}}=1,0202 \quad \nu_{g}^{\prime} m_{g}^{\prime}=13,0331 ;
\end{array}
$$

(c) Conforme expressões dadas em (117), tem-se

$$
\begin{gathered}
x=\frac{\nu_{g}^{\prime} m_{g}^{\prime}}{\nu_{g}^{\prime} m_{g}^{\prime}+\nu_{e} m_{e}}=0,1237, \quad P_{4}=I_{x}(7,3875,31,5)=0,1395, \\
P_{5}=I_{x}(8,3875,31,5)=0,0742, \quad P_{6}=I_{x}(6,3875,31,5)=0,2425,
\end{gathered}
$$

onde

$$
I_{x}(p, q)=\frac{1}{B(p, q)} \int_{0}^{x} u^{p-1}(1-u)^{q-1} d u \quad \text { é a integral de uma Beta. }
$$

(d) Agora, pelas expressões (115), (116), (118), segue que,

$$
\begin{gathered}
c=\left(\frac{\nu_{g}^{\prime}+2}{2}\right) \frac{P_{5}}{P_{4}}-\frac{\nu_{g}^{\prime}}{2} \frac{P_{4}}{P_{6}}=0,2550, \quad d=\frac{\nu_{g}^{\prime}}{c} \frac{P_{4}}{P_{6}}=27,0710=\nu_{g}^{*} \quad e \\
m_{g}^{*}=\frac{\nu_{g}^{\prime} m_{g}^{\prime}}{c \nu_{g}^{*}}=1,8880 .
\end{gathered}
$$

O efeito da restrição $C: \sigma_{e g b}^{2}>\sigma_{e g}^{2}>\sigma_{e}^{2}$, fez com que os graus de liberdade $\nu_{g}=12$ mudassem para $\nu_{g}^{*}=27$ e o quadrado médio da interação $m_{g}=1,0429$ mudasse para $m_{g}^{*}=1,8880$.

\subsubsection{A Distribuição a Posteriori Aproximada de $\phi, p(\phi \mid y)$}

A função de distribuição a posteriori aproximada para os efeitos comparativos dos tratamentos, $\phi$, é dada por,

$$
\begin{aligned}
p(\phi \mid y) \doteq & \frac{\Gamma\left(\frac{\nu_{q}^{*}+\nu_{\dot{\phi}}}{2}\right)\left|\sum\right|^{-\frac{1}{2}}\left(m_{g}^{*}\right)^{-\frac{\nu_{\phi}}{2}}}{\left[\Gamma\left(\frac{1}{2}\right)\right]^{\nu_{\dot{\rho}}} \Gamma\left(\frac{\nu_{g}^{*}}{2}\right)\left(\nu_{g}^{*}\right)^{\frac{\nu_{g}^{*}}{2}}}\left[1+\frac{(\phi-\hat{\phi})^{\prime} \sum^{-1}(\phi-\hat{\phi})}{\nu_{g}^{*} m_{g}^{*}}\right]^{-\frac{\nu_{\phi}+\nu_{\dot{g}}^{*}}{2}}, \\
& -\infty<\phi_{i}<+\infty, \quad i=1,2, \ldots, 6
\end{aligned}
$$


a qual, conforme os contrastes utilizados, pode ser denotada por

$$
\phi \mid y \dot{\sim} t_{6}\left(\left[\begin{array}{r}
21,2625 \\
8,8525 \\
7,3692 \\
-1,0808 \\
-0,2675 \\
2,5192
\end{array}\right] ; \quad \frac{1}{12}\left[\begin{array}{cccccc}
42 & 0 & 0 & 0 & 0 & 0 \\
0 & 30 & 0 & 0 & 0 & 0 \\
0 & 0 & 10 & 0 & 0 & 0 \\
0 & 0 & 0 & 14 & 0 & 0 \\
0 & 0 & 0 & 0 & 10 & 0 \\
0 & 0 & 0 & 0 & 0 & 70
\end{array}\right] 1,8880 ; \quad 27\right) \text {. }
$$

\subsubsection{Inferências Conjunta e Marginais Sobre $\phi$ na Análise Conjunta}

Verificar a hipótese $H_{0}: \phi=\mathbf{0}\left(\theta_{1}=\theta_{2}=\cdots=\theta_{7}=\theta\right)$ a um nível de significância $\alpha$, é equivalente a estudar $p(\phi \mid y)$ e verificar se o ponto zero está incluído na região H.P.D. que contém $(1-\alpha)$ de probabilidade. Neste sentido o ponto zero estará incluído na região H.P.D. de conteúdo $(1-\alpha)$ se, e somente se,

$$
\begin{gathered}
\frac{S\left(\boldsymbol{\phi}_{0}=\mathbf{0}\right)}{(K-1) m_{g}^{*}}=\frac{(\mathbf{0}-\hat{\boldsymbol{\phi}})^{\prime}\left[D\left(X^{\prime} X\right)^{-1} D^{\prime}\right]^{-1}(\mathbf{0}-\hat{\boldsymbol{\phi}})}{(K-1) m_{g}^{*}}= \\
=\frac{1}{(K-1) m_{g}^{*}} \sum_{j=1}^{K-1} \frac{J R\left(\sum_{i=1}^{K} d_{j i} \bar{y}_{i . .}\right)^{2}}{\sum_{i=1}^{k} d_{j i}^{2}}=\frac{J R \sum_{i=1}^{K}\left(\bar{y}_{i . .}-\bar{y}_{\ldots}\right)^{2}}{(K-1) m_{g}^{*}}<F_{\left[(K-1), \nu_{g}^{*}, \alpha\right]} .
\end{gathered}
$$

Para os dados dos três experimentos, encontrou-se

$$
\frac{S(\mathbf{0})}{6 \times 1,8880}=\frac{227,8570}{6 \times 1,8880}=20,11
$$

o qual é maior que $F_{[6,27,0,05]}=2,46$. Logo, o ponto zero está fora da região H.P.D. que contém $95 \%$ de probabilidade, o que é equivalente a rejeitar $H_{0}$ a um nível de significância $\alpha=0,05$.

Para se fazer inferência marginal sobre qualquer contraste $\phi_{j}$, deve-

se usar o fato de que

$$
\phi_{j} \mid \boldsymbol{y} \sim t_{1}\left[\hat{\phi}_{j}, \frac{m_{g}^{*}}{J R} \sum_{i=1}^{K} d_{j i}^{2}, \nu_{g}^{*}\right] .
$$

Verificar a hipótese $H_{0}: \phi_{j}=0$ a um nível de significância $\alpha=0,05$ é equivalente a estudar a densidade posteriori $p\left(\phi_{j} \mid \boldsymbol{y}\right)$ e verificar se o ponto zero 
está dentro ou fora da região H.P.D. que contém $95 \%$ de probabilidade. Neste sentido, o ponto zero estará dentro da região H.P.D. se, e somente se,

$$
\frac{S\left(\phi_{j 0}=0\right)}{m_{g}^{*}}=\frac{\left(\sum_{i=1}^{K} d_{j i} \bar{y}_{i . .}\right)^{2}}{\frac{m_{g}^{*}}{J R} \sum_{i=1}^{K} d_{j i}^{2}}<F_{\left\{1, \nu_{g}^{*}, 0,05\right\}} .
$$

Este resultado é equivalente a verificar se o ponto zero está incluído no intervalo com 95\% de credibilidade. Assim, para os oito contrastes considerados de interesse, encontraram-se os seguintes resultados constantes na tabela 14

Tabela 14: Inferèncias marginais sobre os contrastes de interesse

\begin{tabular}{|c|r|r|c|}
\hline \hline Contraste & \multicolumn{1}{|c|}{$\hat{\phi}_{j}$} & $\frac{S\left(\phi_{j 0}=0\right)}{m_{j}^{*}}$ & $\hat{\phi}_{j} \pm t_{\frac{\alpha}{2}} \sqrt{\frac{\bar{m}_{3}^{*} \sum_{i=1}^{7} d_{j i}^{2}}{J R}}$ \\
\hline \hline$\phi_{1}$ & 21.2625 & 68.4162 & {$[15,99 ; 26,54]$} \\
$\phi_{2}$ & 8.8525 & 16.6031 & {$[4,39 ; 13,31]$} \\
$\phi_{3}$ & 7.3692 & 34.5156 & {$[4,80 ; 9,94]$} \\
$\phi_{4}$ & -1.0808 & 0.5303 & {$[-4,13 ; 1,96]$} \\
$\phi_{5}$ & -0.2675 & 0.0455 & {$[-2,84 ; 2,31]$} \\
$\phi_{6}$ & 2.5192 & 0.5762 & {$[-4,29 ; 9,33]$} \\
\hline$\phi_{7}$ & 2.0683 & 13.5953 & {$[0,92 ; 3,22]$} \\
$\phi_{8}$ & 0.2050 & 0.1336 & {$[-0,95 ; 1,36]$} \\
\hline
\end{tabular}

$$
F_{[1,27,0,05]}=4,21 \quad \text { e } t_{[27,0,05]}=2,052=t_{\frac{\alpha}{2}}
$$

Observa-se que o ponto zero está dentro da região H.P.D. dos contrastes $\dot{\phi}_{4}, \phi_{5}, \phi_{6}$ e $\phi_{8}$, o que é equivalente a aceitar a hipótese de que estes contrastes são todos marginalmente nulos, a um nível de significància $\alpha=0,05$. O ponto zero está fora da região H.P.D. dos contrastes $\phi_{1}, \phi_{2}, \phi_{3}$ e $\phi_{7}$, o que é equivalente a rejeitar a hipótese de que eles são marginalmente nulos a um nível de significância $\alpha=0,05$. O fato do ponto zero não pertencer a região H.P.D. do contraste $\phi_{3}$, 
traduz que o comportamento médio da produção de açúcar em relação aos níveis de Cobre, na presença de NPK + Micronutrientes, é linear.

\subsubsection{Ajuste dos Graus de Liberdade e Quadrados Médios para Cons- trução das Densidades a Posteriori dos Componentes da Variância}

A partir dos dados experimentais e das expressões dadas nas subseções (3.3.9) a (3.3.12), foram calculadas as quantidades necessárias para determinação das densidades marginais a posteriori aproximadas dos componentes da variância $\left(\sigma_{e}^{2}, \sigma_{g}^{2}, \sigma_{b}^{2}\right)$. Foi utilizado o software MATHEMATICA na determinação dessas quantidades, as quais encontram-se na tabela 15.

Tabela 15: Elementos Básicos usados para estabelecer as distribuições marginais a posteriori dos componentes da variância.

\begin{tabular}{rrr}
\hline$P_{7}=0,229145$ & $P_{8}=0,228928$ & $P_{9}=0,229468$ \\
$\nu_{e}^{*}=62,068900$ & $m_{e}^{*}=1,407050$ & $\nu_{e}^{*} m_{e}^{*}=91,058200$ \\
\hline$P_{1}=0,966400$ & $P_{2}=0,979300$ & $P_{3}=0,945400$ \\
$\nu_{g}^{\prime}=12,775000$ & $m_{g}^{\prime}=1,020200$ & $\nu_{g}^{\prime} m_{g}^{\prime}=13,033100$ \\
\hline$P_{10}=0,263400$ & $P_{11}=0,284800$ & $P_{12}=0,242500$ \\
$\nu_{e}^{\prime}=74,000000$ & $m_{e}^{\prime}=1,348800$ & $\nu_{e}^{\prime} m_{e}^{\prime}=97,712800$ \\
\hline$P_{13}=0,153313$ & $P_{14}=0,081412$ & $P_{15}=0,265722$ \\
$\nu_{g} ”=27,119700$ & $m_{g} "=1,807500$ & $\nu_{g} " m_{g} "=49,018900$ \\
\hline \hline
\end{tabular}

\subsubsection{Inferência Marginal a Posteriori Sobre $\sigma_{e}^{2}$}

No desenvolvimento teórico, foram apresentadas quatro modalidades para densidade a posteriori de $\sigma_{e}^{2}, p\left(\sigma_{e}^{2} \mid \mathbf{y}\right)$ :

$p_{1}=$ densidade a posteriori de $\sigma_{e}^{2}$ aproximada em um estágio,

$p_{2}=$ densidade a posteriori de $\sigma_{e}^{2}$ aproximada em dois estágios, 
$p_{i}=$ densidade a posteriori $\operatorname{de} \sigma_{e}^{2}$ irrestrita,

$p_{c}=$ densidade a posteriori de $\sigma_{e}^{2}$ composta (pooling).

Em geral, as inferências sobre $\sigma_{e}^{2}$ são feitas com base na densidade a posteriori aproximada em dois estágios, (ver BoX \& TiaO(1973)). Porém, para visualizar o comportamento destas densidades e comparar as inferências sobre $\sigma_{e}^{2}$ a partir de cada uma delas, foram determinadas suas densidades, traçados seus gráficos (figuras 2 e 3), calculadas algumas características das distribuições e intervalos com $95 \%$ de credibilidade para $\sigma_{e}^{2}$, conforme mostra a tabela 16 . Isto é, pelas expressões (139), (151), (152) e (154), tem-se que,

$$
\begin{aligned}
& p_{1} \doteq p_{1}\left(\sigma_{e}^{2} \mid \mathbf{y}\right)=\frac{(91,0582)^{31}}{\Gamma(31) 2^{31}}\left(\sigma_{e}^{2}\right)^{-32} e^{-\frac{45,5221}{\sigma_{e}^{2}}} I_{(0, \infty)}\left(\sigma_{e}^{2}\right), \\
& p_{2} \doteq p_{2}\left(\sigma_{e}^{2} \mid \mathbf{y}\right)=\frac{(99,7128)^{37}}{\Gamma(37) 2^{37}}\left(\sigma_{e}^{2}\right)^{-38} e^{-\frac{49,8564}{\sigma_{e}^{2}}} I_{(0, \infty)}\left(\sigma_{e}^{2}\right), \\
& p_{i}=p_{i}\left(\sigma_{e}^{2} \mid \mathbf{y}\right)=\frac{(92,2941)^{31,5}}{\Gamma(31,5) 2^{31,5}}\left(\sigma_{e}^{2}\right)^{-32,5} e^{-\frac{46,14705}{\sigma_{e}^{2}}} I_{(0, \infty)}\left(\sigma_{e}^{2}\right), \\
& p_{c} \doteq p_{c}\left(\sigma_{e}^{2} \mid \mathbf{y}\right)=\frac{(100,2065)^{37}}{\Gamma(37) 2^{37}}\left(\sigma_{e}^{2}\right)^{-38} e^{-\frac{50,10325}{\sigma_{e}^{2}}} I_{(0, \infty)}\left(\sigma_{e}^{2}\right),
\end{aligned}
$$

cujos gráficos individuais destas densidades encontram-se na figura 2 e um gráfico conjunto das quatro densidades na figura 3.

A tabela 16 apresenta as principais características da distibuição $a$ posteriori de $\sigma_{e}^{2}$ relativas a cada aproximação.

Os resultados constantes da tabela 16 foram obtidos por definição de cada característica a partir das respectivas densidades a posteriori e empregando o software MATHEMATICA, cujos comandos podem ser vistos no Apêndice.

Com base na figura 3 e tabela 16, observa-se que as densidades $a$ posteriori aproximada em um estágio $\left(p_{1}\right)$ e a irrestrita $\left(p_{i}\right)$, apresentaram resultados semelhantes, porém ligeiramente diferentes das densidades a posteriori aproximada em dois estágios $\left(p_{2}\right)$ e composta (pooling) $\left(p_{c}\right)$, as quais, também, apresentaram resultados semelhantes entre si. 


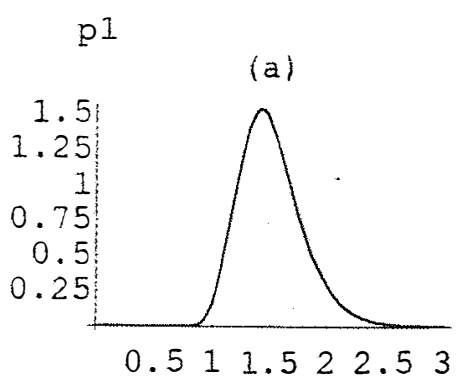

pi

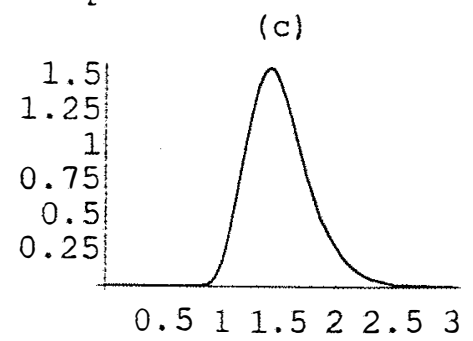

$\mathrm{p} 2$

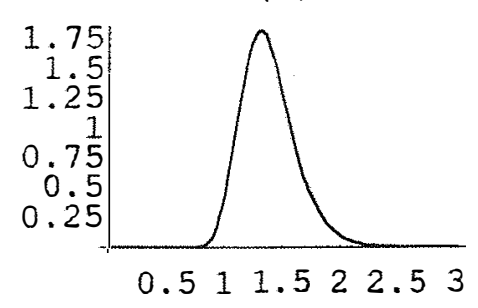

pc

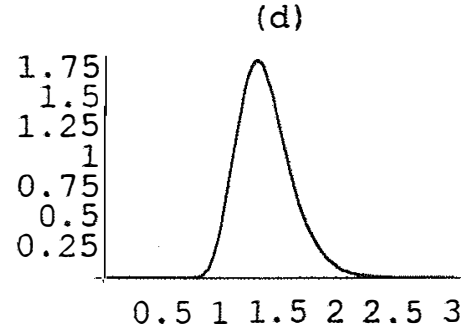

Figura 2: Gráficos individuais das densidades a posteriori de $\sigma_{e}^{2}$.

\subsubsection{Inferência Marginal a Posteriori Sobre $\sigma_{g}^{2}$}

Baseado nas suposições e nos dados dos três ensaios, a densidade marginal a posteriori aproximada do componente da variância $\sigma_{g}^{2}$ obtida a partir da expressão (157), foi

$$
p\left(\sigma_{g}^{2} \mid \mathbf{y}\right) \doteq \frac{4(13,0331)^{6,3875}}{\Gamma(6,3875) 2^{6,3875}(0,233744)}\left(1,4650+4 \sigma_{g}^{2}\right)^{-7,3875} e^{-\frac{13,0331}{2,93+8 \sigma_{g}^{2}}} I_{(0, \infty)}\left(\sigma_{g}^{2}\right),
$$

com as seguintes características:

$$
\operatorname{Var}\left[\sigma_{g}^{2} \mid \mathbf{y}\right]=0,0242, \quad E\left[\sigma_{g}^{2} \mid \mathbf{y}\right]=0,1357 ; \quad M o\left[\sigma_{g}^{2} \mid \mathbf{y}\right]=0, \quad M d\left[\sigma_{g}^{2} \mid \mathbf{y}\right]=0,0875
$$

O gráfico da distribuição a posteriori de $\sigma_{g}^{2}$, encontra-se na figura 4 e o intervalo com $95 \%$ de credibilidade para $\sigma_{g}^{2}$ obtido a partir da expressão 158 , foi

$$
\operatorname{Pr}\left\{0<\sigma_{g}^{2}<0,4248 \mid \mathbf{y}\right\} \doteq 0,95
$$




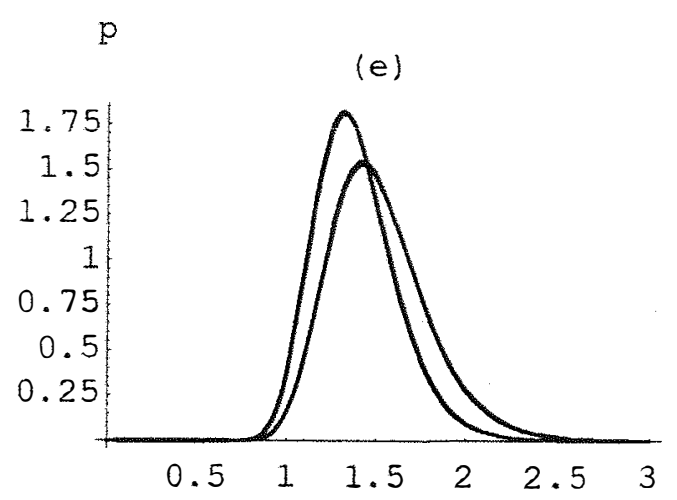

Figura 3: Gráfico conjunto das posteriori's aproximadas de $\sigma_{e}^{2}$.

Tabela 16: Características da distribuição a posteriori de $\sigma_{e}^{2}$ para as diferentes aproximações.

\begin{tabular}{cccccc}
\hline \hline Posteriori & Variância & Média & Moda & Mediana & $I C\left(\sigma_{e}^{2}\right)_{(95 \%)}$ \\
\hline \hline$p_{1}$ & 0,07942 & 1,51764 & 1,42278 & 1,4848 & {$[1,0632 ; 2,1620]$} \\
$p_{2}$ & 0,05480 & 1,38490 & 1,31201 & 1,3598 & {$[1,0004 ; 1,9140]$} \\
$p_{i}$ & 0,07760 & 1,51302 & 1,41991 & 1,4808 & {$[1,0630 ; 2,1491]$} \\
$p_{c}$ & 0,05534 & 1,39176 & 1,31851 & 1,3666 & {$[1,0054 ; 1,9236]$} \\
\hline \hline
\end{tabular}

\subsubsection{Inferência Marginal a Posteriori Sobre $\sigma_{b}^{2}$}

Considerando-se as suposiçōes e os dados dos ensaios, a densidade marginal a posteriori aproximada, obtida para o componente da variância devido ao fator experimento $\sigma_{b}^{2}, p\left(\sigma_{b}^{2} \mid \mathbf{y}\right)$, foi, conforme expressão (168),

$$
\begin{aligned}
& p\left(\sigma_{b}^{2} \mid \mathbf{y}\right) \doteq(123,5180)\left(1,8075+28 \sigma_{b}^{2}\right)^{-2} e^{-\frac{7.8040}{3,6150+56 \sigma_{b}^{2}}} I_{(0, \infty)}\left(\sigma_{b}^{2}\right) . \\
& \text { O gráfico da distribuição encontra-se na figura } 5 \text { e as características }
\end{aligned}
$$
encontradas foram:

$$
E\left[\sigma_{b}^{2} \mid \mathbf{y}\right]=1,2371, \quad M o\left[\sigma_{b}^{2} \mid \mathbf{y}\right]=0,0051, \quad M d\left[\sigma_{b}^{2} \mid \mathbf{y}\right]=0,17413
$$




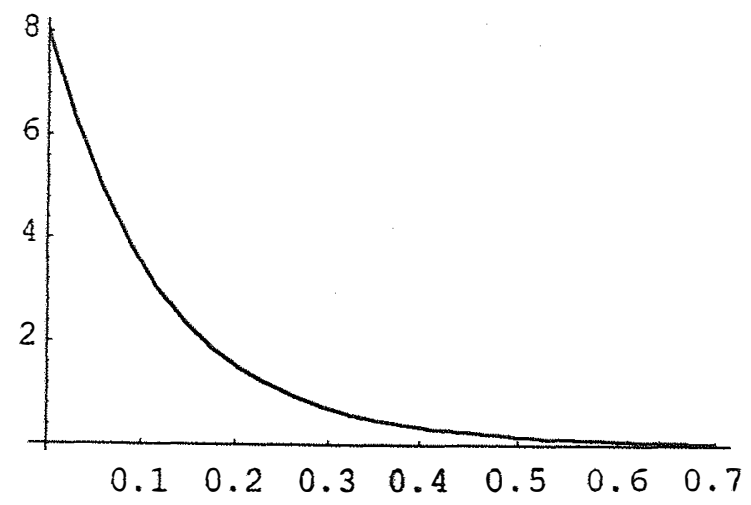

Figura 4: Gráfico da densidade marginal a posteriori de $\sigma_{g}^{2}$.

Figura 5: Gráfico da densidade marginal a posteriori de $\sigma_{b}^{2}$.

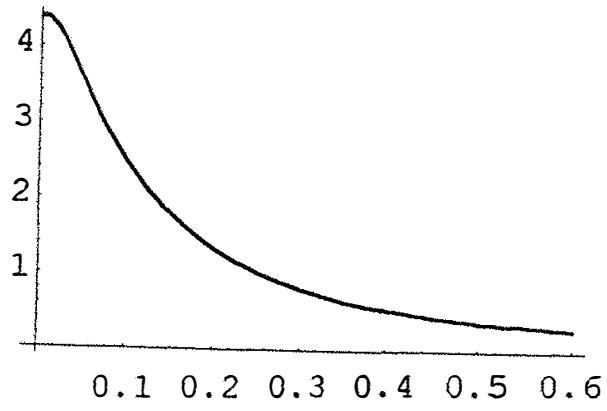

e o intervalo com $95 \%$ de credibilidade para $\sigma_{b}^{2}$, obtido a partir da expressão (169), foi

$$
\operatorname{Pr}\left\{0<\sigma_{b}^{2}<3,0163 \mid y\right\} \doteq 0,95
$$

Observa-se que, sendo $p\left(\sigma_{e}^{2} \mid \mathbf{y}\right), p\left(\sigma_{g}^{2} \mid \mathbf{y}\right)$ e $p\left(\sigma_{b}^{2} \mid \mathbf{y}\right)$ densidades de probabilidade definidas no intervalo $(0, \infty)$, isto indica que os componentes da variância $\sigma_{e}^{2}, \sigma_{g}^{2}$ e $\sigma_{b}^{2}$, não são definidos para valores negativos, ou seja, a posteriori, a probabilidade de se obter um valor negativo para qualque componente da variância é zero. Portanto, a análise Bayesiana proposta neste trabalho, não apresenta problemas de componentes da variância negativos. 
Para se obter os gráficos das densidades marginais a posteriori e determinar suas características, foi utilizado o software MATHEMATICA, cujas sequèncias de comandos, encontram-se detalhadamente no Apêndice.

\subsection{A Análise Ċlássica}

Para efeito de comparação das metodologias de análise, achou-se conveniente analisar os dados experimentais através da inferência clássica. Sendo este enfoque bastante conhecido, a abordagem teórica não foi apresentada neste trabalho.

\subsubsection{As Análises Individuais Clássicas}

A análise de variância exige, além de outras suposições, a homogeneidade das variâncias dentro de cada experimento. Esta hipótese poderá ser verificada pelo teste de Bartlett de modo semelhante ao que foi feito na análise Bayesiana, o qual mostrou que existe a homocedasticidade dentro dos très ensaios.

As tabelas 17, 18 e 19, a presentam os resultados das análises de variância dos ensaios individuais, associadas às hipóteses de interesse. Neste sentido, observa-se, portanto, que a hipótese de igualdade dos efeitos médios dos tratamentos, $H_{0}^{(1)}: \theta_{1}=\ldots=\theta_{7}=\theta$, foi rejeitada a nível $\alpha=0,05$, em todos os ensaios. Os

contrastes $\phi_{1}=-\theta_{1}+\frac{\theta_{2}+\ldots+\theta_{I}}{6}$ e $\phi_{2}=-\theta_{2}+\frac{\theta_{3}+\ldots+\theta_{7}}{5}$ e o componente de regressão polinomial de $1^{0}$ grau $\phi_{3}$, apresentaram significância estatística ao nível de $5 \%$ de probabilidade pelo teste $F$, nos très ensaios. Por outro lado, a menos do experimento instalado na Usina São José (E3), o contraste $\phi_{7}=-\theta_{2}+\theta_{3}$ e os componentes de regressão de $2^{0}$ a $4^{0}$ graus, não apresentaram significância estatística ao nível de $\alpha=0,05$ pelo teste F. Estes resultados,

obtidos pela análise clássica, foram exatamente os mesmos daqueles obtidos via enfoque Bayesiano. Estas conclusões já eram esperadas, tendo em vista que na análise Bayesiana, usou-se uma priori não-informativa para os parâmetros $\left(\boldsymbol{\theta}, \sigma^{2}\right)$ e o modelo estatístico $\boldsymbol{y}=\boldsymbol{X} \boldsymbol{\theta}+\epsilon$, foi considerado de efeito fixo. 
Nas análises individuais, inferèncias sobre a variància do erro experimental $\sigma^{2}$, são feitas com base no fato de que $\sum_{i=1}^{K}\left(y_{i j}-\bar{y}_{i .}\right)^{2} / \sigma^{2} \sim \chi_{\nu}^{2}$, onde $\nu=$ $K(R-1)$. Assim, conforme SEARLE et all (1992, pg. 135-136) e Graybill(1976, pg. 624-625) os intervalos de confiança para $\sigma^{2}$ com $100(1-\alpha) \%$ de confiança, poderão ser construídos como segue.

$$
\text { I.C. }\left(\sigma^{2}\right)_{(1-\alpha)}: \frac{S \cdot Q \cdot R e s .}{\chi_{\nu, S}^{2}} \leq \sigma^{2} \leq \frac{S \cdot Q \cdot R e s .}{\chi_{\nu, I}^{2}}
$$

onde, $\chi_{\nu, I}^{2}$ e $\chi_{\nu, S}^{2}$ sào tais que

$$
\operatorname{Pr}\left\{\chi_{\nu, I}^{2} \leq \chi_{\nu}^{2} \leq \chi_{\nu, S}^{2}\right\}=1-\alpha
$$

Para os três experimentos envolvidos no estudo, encontrou-se: para $\nu=K(R-1)=21$ e $\alpha=0,05, \chi_{\nu, I}^{2}=10,656$ e $\chi_{\nu, S}^{2}=36,525$. Assim, as estimativas da variància do erro experimental e os respectivos intervalos de confiança com $95 \%$ de confiança para cada ensaio, foram respectivamente,

$$
\begin{aligned}
& \hat{\sigma}_{1}^{2}=1,3336 \text { e } \quad \text { I.C. }\left(\sigma_{1}^{2}\right)_{(0,95)}:[0,7668 ; 2,6282], \\
& \hat{\sigma}_{2}^{2}=2,5176 \text { e } \quad \text { I.C. }\left(\sigma_{2}^{2}\right)_{(0,95)}:[1,4475 ; 4,9616], \\
& \hat{\sigma}_{3}^{2}=0,5437 \text { e } \quad \text { I.C. }\left(\sigma_{3}^{2}\right)_{(0,95)}:[0,3126 ; 1,0715] .
\end{aligned}
$$

É importante lembrar, que apesar dos limites dos intervalos de confiança coincidirem com os limites dos intervalos de credibilidade dados na seção 4.3.2, os dois tipos de intervalos são distintos na interpretação.

\subsubsection{A Análise Conjunta Clássica}

Os resultados da análise de variância conjunta, encontram-se na tabela 20. Nessa, pode-se observar, que a hipótese de igualdade dos efeitos médios dos tratamentos, $H_{0}^{(1)}: \theta_{1}=\ldots=\theta_{T}=\theta$, foi rejeitada ao nível de significància $\alpha=0,05$. Os contrastes $\phi_{1}=-\theta_{1}+\frac{\theta_{2}+\ldots+\theta_{7}}{6}, \phi_{2}=-\theta_{2}+\frac{\theta_{3}+\ldots+\theta_{7}}{5}, \phi_{7}=-\theta_{1}+\theta_{2} \mathrm{e}$ o componente de regressão linear $\phi_{3}$, apresentaram significància estatística ao nível 
Tabela 17: Resultados da análise de variância relativa aos dados do experimento instalado na Usina N. S. Maravilhas

\begin{tabular}{lrrrrr}
\hline F. Variação & G.L. & S.Q. & Q.M. & F & Pr > F \\
\hline T1 vs (T2+...T7) & 1 & 19,3936 & 19,3936 & 14,54 & 0,0010 \\
T2 vs (T3+...T7) & 1 & 8,4376 & 8,4376 & 6,33 & 0,0201 \\
Comp. de 1o. Grau & 1 & 31,2229 & 31,2229 & 23,41 & 0,0001 \\
Comp. de 2o. Grau & 1 & 1,4208 & 1,4208 & 1,07 & 0,3137 \\
Comp. de 3o. Grau & 1 & 0,2924 & 0,2924 & 0,22 & 0,6444 \\
Comp. de 4o. Grau & 1 & 0,5075 & 0,5075 & 0,38 & 0,5440 \\
\hline Tratamento & 6 & 61,2748 & 10,2125 & 7,66 & 0,0002 \\
Resíduo & 21 & 28,0063 & 1,3336 & - & - \\
\hline Total & 27 & 89,2811 & & - & \\
\hline \hline
\end{tabular}

$\alpha=0,05$ pelo teste $\mathrm{F}$. Em contrapartida, o contraste $\phi_{8}=-\theta_{2}+\theta_{3}$ e os componentes da regressão de $2^{0}$ a $4^{0}$ graus não apresentaram significância estatística.

Embora estas conclusões sejam as mesmas daquelas obtidas pela análise Bayesiana, isto nem sempre deverá ocorrer, uma vez que na análise Bayesiana há o efeito do fator modificador $g(\phi)$ induzido pela restrição $C: \sigma_{e g b}^{2}>\sigma_{e g}^{2}>\sigma_{e}^{2}$, que leva a resultados diferentes daqueles obtidos pela análise clássica.

Conforme SEARLE et all (1992, pg. 135-136) e GraYBILL(1976, pg. 624-625), os estimadores dos componentes da variância, pelo método da ANOVA, associados ao erro experimental, interação $T \times E$ e ao fator experimento, são dados 
Tabela 18: Resultados da análise de variância relativa aos dados do experimento instalado na Usina Santa Tereza

\begin{tabular}{lrrrrr}
\hline F. Variação & G.L. & S.Q. & Q.M. & F & Pr $>$ F \\
\hline T1 vs (T2+...T7) & 1 & 62,8426 & 62,8426 & 24,96 & 0,0001 \\
T2 vs (T3+...T7) & 1 & 19,2721 & 19,2721 & 7,65 & 0,0116 \\
Comp. de 1o. Grau & 1 & 22,0820 & 22,0820 & 8,77 & 0,0074 \\
Comp. de 2o. Grau & 1 & 0,0645 & 0,0645 & 0,03 & 0,8744 \\
Comp. de 3o. Grau & 1 & 0,1145 & 0,1145 & 0,05 & 0,8332 \\
Comp. de 4o. Grau & 1 & 0,1872 & 0,1872 & 0,07 & 0,7878 \\
\hline Tratamento & 6 & 104,5629 & 17,4271 & 6,92 & 0,0004 \\
Resíduo & 21 & 52,8705 & 2,5176 & & \\
\hline Total & 27 & 157,4333 & & & \\
\hline \hline
\end{tabular}

por.

$$
\hat{\sigma}_{e}^{2}=m_{e}, \quad \hat{\sigma}_{g}^{2}=\frac{m_{g}-m_{e}}{R} \quad e \quad \hat{\sigma}_{b}^{2}=\frac{m_{b}-m_{g}}{K R} .
$$

Além disso, os intervalos de confiança com $100(1-\alpha) \%$ de confiança para os componentes da variância $\sigma_{e}^{2}, \sigma_{g}^{2}$ e $\sigma_{b}^{2}$, serão obtidos com base nos seguites argumentos:

(a) As quantidades $\frac{\nu_{e} m_{s}}{\sigma_{\varepsilon}^{2}} \cdot \frac{\nu_{g} m_{g}}{R \sigma_{g}^{2}+\sigma_{s}^{2}}$ e $\frac{\nu_{b} m_{b}}{K R \sigma_{b}^{2}+R \sigma_{g}^{2}+\sigma_{\varepsilon}^{2}}$ são distribuidas exatamente como:

$$
\frac{\nu_{e} m_{e}}{\sigma_{e}^{2}} \sim \chi_{\nu_{s}}^{2}, \quad \frac{\nu_{g} m_{g}}{R \sigma_{g}^{2}+\sigma_{e}^{2}} \sim \chi_{\nu_{g}}^{2} \quad e \quad \frac{\nu_{b} m_{b}}{K R \sigma_{b}^{2}+R \sigma_{g}^{2}+\sigma_{e}^{2}} \sim \chi_{\nu_{b}}^{2}
$$

(b) Sejam $\nu_{i}$ os graus de liberdade. $m_{i}$ o quadrado médio e $S_{i}$ a soma de quadrados 
Tabela 19: Resultados da análise de variância relativa aos dados do experimento instalado na Usina São José

\begin{tabular}{lrrrrr}
\hline F. Variação & G.L. & S.Q. & Q.M. & F & Pr $>$ F \\
\hline \hline T1 vs (T2+...T7) & 1 & 54,0828 & 54,0828 & 99,47 & 0,0001 \\
T2 vs (T3+...T7) & 1 & 5,7728 & 5,7728 & 10,62 & 0,0038 \\
Comp. de 1o. Grau & 1 & 13,6539 & 13,6539 & 25,11 & 0,0001 \\
Comp. de 2o. Grau & 1 & 0,0825 & 0,0825 & 0,15 & 0,7007 \\
Comp. de 3o. Grau & 1 & 0,5040 & 0,5040 & 0,93 & 0,3466 \\
Comp. de 4o. Grau & 1 & 0,4377 & 0,4377 & 0,80 & 0,3798 \\
\hline Tratamento & 6 & 74,5337 & 12,4223 & 22,85 & 0,0001 \\
Resíduo & 21 & 11,4174 & 0,5437 & & \\
\hline Total & 27 & 85,9511 & & & \\
\hline \hline
\end{tabular}

\begin{tabular}{lrrrrr}
\hline F. Variação & G.L. & S.Q. & Q.M. & F & Pr $>$ F \\
\hline \hline T1 vs T2 & 1 & 16,5313 & 16,5313 & 30,41 & 0,0001 \\
T2 vs T3 & 1 & 0,0990 & 0,0990 & 0,18 & 0,6739 \\
Resíduo & 21 & 11,4174 & 0,5437 & & \\
\hline \hline
\end{tabular}

do fator i. Então,

$$
\text { I.C. }\left(E\left[m_{i}\right]\right)_{(1-\alpha)}: \quad \frac{\nu_{i} m_{i}}{\chi_{\nu_{i}, S}^{2}} \leq E\left[m_{i}\right] \leq \frac{\nu_{i} m_{i}}{\chi_{\nu_{i}, I}^{2}}
$$

onde $\chi_{\nu_{i}, I}^{2}$ e $\chi_{\nu_{i}, S}^{2}$ são tais que

$$
\operatorname{Pr}\left\{\chi_{\nu_{i}, I}^{2} \leq \chi_{\nu_{i}}^{2} \leq \chi_{\nu_{i}, S}^{2}\right\}=1-\alpha
$$

(c) Seja a razão das esperanças de dois quadrados médios $E\left[m_{i}\right] / E\left[m_{\cdot j}\right]$. Então.

$$
\text { I.C. }\left(E\left[m_{i}\right] / E\left[m_{j}\right]\right)_{(1-\alpha)}: \quad \frac{m_{i}}{m_{j}} F_{\left[\nu_{j}, \nu_{i}, I\right]} \leq \frac{E\left[m_{i}\right]}{E\left[m_{j}\right]} \leq \frac{m_{i}}{m_{j}} F_{\left[\nu_{j}, \nu_{i}, S\right]},
$$


Tabela 20: Resultados da análise de variância conjunta.

\begin{tabular}{lrrrrr}
\hline F. Variação & G.L. & S.Q. & Q.M. & F & Pr > F \\
\hline \hline T1 vs (T2+...T7) & 1 & 129,1697 & 129,1697 & 123,86 & 0,0001 \\
T2 vs (T3+...T7) & 1 & 31,3467 & 31,3467 & 30,06 & 0,0001 \\
Comp. de 1o. Grau & 1 & 65,1655 & 65,1655 & 62,49 & 0,0001 \\
Comp. de 2o. Grau & 1 & 1,0013 & 1,0013 & 0,96 & 0,3465 \\
Comp. de 3o. Grau & 1 & 0,0859 & 0,0859 & 0,08 & 0,7790 \\
Comp. de 4o. Grau & 1 & 1,0879 & 1,0879 & 1,04 & 0,3272 \\
\hline Tratamento(T) & 6 & 227,8570 & 37,9762 & 36,42 & 0,0001 \\
Experimento(E) & 2 & 7,8040 & 3,9020 & 3,74 & 0,0546 \\
Interação $T \times E$ & 12 & 12,5143 & 1,0429 & 0,71 & 0,7343 \\
Resíduo & 63 & 92,2941 & 1,4650 & & \\
\hline Total & 83 & 340,4695 & & & \\
\hline \hline & \multicolumn{7}{c}{ GL } & S.Q. & QM & F & Pr > F \\
\hline F. Variação & 1 & 25,6680 & 25,6680 & 24,61 & 0,0003 \\
\hline \hline T1 vs T2 & 1 & 0,2522 & 0,2522 & 0,24 & 0,6318 \\
T2 vs T3 & 12 & 12,5143 & 1,0429 & & \\
Intaração T $\times E$ & GL & & & \\
\hline \hline
\end{tabular}

onde $F_{\left[\nu_{1}, \nu_{i}, I\right]}$ e $F_{\left[\nu_{j}, \nu_{i}, S\right]}$, são tais que

$$
\operatorname{Pr}\left\{F_{\left[\nu_{j}, \nu_{i}, I\right]} \leq F_{\left[\nu_{j}, \nu_{i}\right]} \leq F_{\left[\nu_{j}, \nu_{i}, S\right]}\right\}=1-\alpha
$$

(d) Suponha, ainda, que $E\left[m_{i}\right]$ difira da $E\left[m_{j}\right]$ apenas por um componente $\sigma^{2}$ (um dos múltiplos componentes), isto é, $E\left[m_{i}\right]=k \sigma^{2}+E\left[m_{j}\right]$. Então, um intervalo de confiança para $\sigma^{2} \operatorname{com}(1-2 \alpha)$ de confiança, será dado, de modo 
aproximado, por

$$
\text { I.C. }\left(\sigma^{2}\right)_{(1-2 \alpha)}: \quad \frac{\nu_{i} m_{i}\left(1-\frac{m_{j}}{m_{i}} F_{\left(\nu_{i}, \nu_{j}, S\right]}\right)}{k \chi_{\nu_{i}, S}^{2}} \leq \sigma^{2} \leq-\frac{\nu_{i} m_{i}\left(1-\frac{m_{j}}{m_{i}} F_{\left(\nu_{i}, \nu_{j}, I\right]}\right)}{k \chi_{\nu_{i}, I}^{2}}
$$

onde $\chi_{\nu_{i}, I}^{2}, \chi_{\nu_{i}, S}^{2}, F_{\left[\nu_{i}, \nu_{j}, I\right]}$ e $F_{\left[\nu_{i}, \nu_{j}, S\right]}$, são tais que

$$
\operatorname{Pr}\left\{\chi_{\nu_{i}, I}^{2} \leq \chi_{\nu_{i}}^{2} \leq \chi_{\nu_{i}, S}^{2}\right\}=1-\alpha
$$

e

$$
\operatorname{Pr}\left\{F_{\left[\nu_{i}, \nu_{j}, I\right]} \leq F_{\left[\nu_{i}, \nu_{j}\right]} \leq F_{\left[\nu_{i}, \nu_{j}, S\right]}\right\}=1-\alpha
$$

Desse modo, um intervalo de confiança para $\sigma_{e}^{2}$, com $100(1-\alpha) \%$ de confiança, será dado, conforme expressão (174), por

$$
\text { I.C. }\left(\sigma_{e}^{2}\right)_{(1-\alpha)}: \quad \frac{\nu_{e} m_{e}}{\chi_{\nu_{e}, S}^{2}} \leq \sigma_{e}^{2} \leq \frac{\nu_{e} m_{e}}{\chi_{\nu_{e}, I}^{2}}
$$

onde, da análise conjunta, $\nu_{e}=63, m_{e}=1,4650$ e $\nu_{e} m_{e}=92,2941$. Admitindo-se $\alpha=0,05$ e utilizando-se o software MATHEMATICA, encontrou-se

$$
\operatorname{Pr}\left\{\chi_{63, I}^{2} \leq \chi_{63}^{2} \leq \chi_{63, S}^{2}\right\}=0,95
$$

onde $\chi_{63, I}^{2}=42,9503$ e $\chi_{63, S}^{2}=86,8296$. Portanto, a estimativa e o intervalo com $95 \%$ de confiança para $\sigma_{e}^{2}$, encontrados, foram respectivamente,

$$
\hat{\sigma}_{e}^{2}=1,4650 \quad e \quad I . C .\left(\sigma_{e}^{2}\right)_{(0,95)}: \quad 1,0629 \leq \sigma_{e}^{2} \leq 2,1489 .
$$

Sendo $E\left[m_{e}\right]=\sigma_{e}^{2}, E\left[m_{g}\right]=R \sigma_{g}^{2}+E\left[m_{e}\right]=R \sigma_{g}^{2}+\sigma_{e}^{2}$ e $E\left[m_{b}\right]=$ $K R \sigma_{b}^{2}+E\left[m_{g}\right]=K R \sigma_{b}^{2}+R \sigma_{g}^{2}+\sigma_{e}^{2}$, então, intervalos de confiança para $\sigma_{g}^{2}$ e $\sigma_{b}^{2}$ com $(1-2 \alpha)$ de confiança, podem ser obtidos, a partir da expressão (177), do seguinte modo.

$$
\text { I.C. }\left(\sigma_{g}^{2}\right)_{(1-2 \alpha)}: \quad \frac{\nu_{g} m_{g}\left(1-\frac{m_{\varepsilon}}{m_{g}} F_{\left[\nu_{g}, \nu_{e}, S\right]}\right)}{R \chi_{\nu_{g}, S}^{2}} \leq \sigma_{g}^{2} \leq \frac{\nu_{g} m_{g}\left(1-\frac{m_{e}}{m_{g}} F_{\left[\nu_{g}, \nu_{e}, I\right]}\right)}{R \chi_{\nu_{g}, I}^{2}}
$$

e

$$
\text { I.C. }\left(\sigma_{b}^{2}\right)_{(1-2 \alpha)}: \quad \frac{\nu_{b} m_{b}\left(1-\frac{m_{g}}{m_{b}} F_{\left[\nu_{b}, \nu_{g}, S\right]}\right)}{K R \chi_{\nu_{b}, S}^{2}} \leq \sigma_{b}^{2} \leq \frac{\nu_{b} m_{b}\left(1-\frac{m_{g}}{m_{b}} F_{\left[\nu_{b}, \nu_{g}, I\right]}\right)}{K R \chi_{\nu_{b}, I}^{2}} .
$$


Da análise conjunta, tem-se que $K=7, R=4, \nu_{g}=12, m_{g}=$ $1,0429, \nu_{g} m_{g}=12,5143, \nu_{e}=63, m_{e}=1,4650, \nu_{e} m_{e}=92,2941, \nu_{b}=2$, $m_{b}=3,9020$ e $\nu_{b} m_{b}=7,8040$. Admitindo-se um nível de significância $\alpha=0,025 \mathrm{e}$ utilizando-se o software MATHEMATICA, encontraram-se os seguintes resultados:

$$
\operatorname{Pr}\left\{\chi_{12, I}^{2} \leq \chi_{12}^{2} \leq \chi_{12, S}^{2}\right\}=0,975,
$$

onde $\chi_{12, I}^{2}=3,7525$ e $\chi_{12, S}^{2}=25,5305$,

$$
\operatorname{Pr}\left\{\chi_{2, I}^{2} \leq \chi_{2}^{2} \leq \chi_{2 . S}^{2}\right\}=0,975,
$$

onde $\chi_{2, I}^{2}=0,02516$ e $\chi_{2, S}^{2}=8,7640$,

$$
\operatorname{Pr}\left\{F_{[12,63, I]} \leq F_{[12,63]} \leq F_{[12,63, S]}\right\}=0,975
$$

onde $F_{[12,63, I]}=0,2985$ e $F_{[12,63, S\}}=2,4024$,

$$
\operatorname{Pr}\left\{F_{[2,12, I]} \leq F_{[2,12]} \leq F_{[2,12, S]}\right\}=0,975
$$

onde $F_{\{2,12, I]}=0,012592$ e $F_{[2,12, S]}=6,454700$. Consequentemente, as estimativas e os intervalos com $95 \%$ de confiança para $\sigma_{g}^{2}$ e $\sigma_{b}^{2}$, encontrados, foram respectivamente,

$$
\begin{array}{cccc}
\hat{\sigma}_{g}^{2}=-0,1055 \quad \text { e } & \text { I.C. }\left(\sigma_{g}^{2}\right)_{(0,95)}: & -0,291007 \leq \sigma_{g}^{2} \leq 0,484136 ; \\
\hat{\sigma}_{b}^{2}=0,1021 \quad \text { e } & \text { I.C. }\left(\sigma_{b}^{2}\right)_{(0,95)}: & -0,023062 \leq \sigma_{b}^{2} \leq 11,040392 .
\end{array}
$$

Observa-se que as estimativas dos componentes da variância $\hat{\sigma}_{e}^{2}, \hat{\sigma}_{g}^{2}$ e $\hat{\sigma}_{b}^{2}$, obtidos pelo método da ANOVA, não coincidiram com nenhuma das características (média, moda, mediana) das distribuições marginais posteriori $p\left(\sigma_{e}^{2} \mid \boldsymbol{y}\right)$, $p\left(\sigma_{b}^{2} \mid \boldsymbol{y}\right)$ e $p\left(\sigma_{b}^{2} \mid \boldsymbol{y}\right)$ dadas pela metodologia Bayesiana. Além disso, o método da ANOVA permitiu que a estimativa do componente da variância $\sigma_{g}^{2}$, assumisse um valor negativo $\left(\hat{\sigma}_{g}^{2}=-0,1055\right)$, resultado que, a priori, não é possível de ser imaginado para uma variância. 
Os limites dos intervalos com $95 \%$ de confiança para $\sigma_{g}^{2}$ e $\sigma_{b}^{2}$, obtidos pela metodologia clássica, além de terem apresentado valores diferentes dos intervalos de credibilidade, apresentaram, também, limites inferiores negativos, os quais são resultados discutíveis.

Os limites dos intervalos com $95 \%$ de credibilidade para $\sigma_{e}^{2}$, obtidos a partir das densidades posteriori aproximadas em um estágio, dois estágios e composta, apresentaram resultados diferentes daqueles obtidos para o intervalo com $95 \%$ de confiança de $\sigma_{e}^{2}$. Por outro lado, os limites do intervalo com $95 \%$ de confiança para $\sigma_{e}^{2}$, coincidiram com aqueles obtidos para o intervalo de credibilidade no modelo irrestrito (ver tabela 16). Este não foi um resultado surpresa, pois, quando não se impõe nenhuma restrição aos parâmetros do modelo e além disso se trabalha com uma priori não-informativa, os resultados das análises, obtidos pela metodologia Bayesiana, coincidem com os resultados obtidos pela inferência clássica, porém com interpretações diferentes. O pior resultado de uma análise Bayesiana é aquele que é obtido quando nenhum conhecimento a priori, acerca dos parâmetros sobre os quais se deseja fazer inferências, foi incorporado na análise. Apesar disso, os resultados da análise Bayesiana são mais fáceis de serem interpretados e permite que certas probabilidades a posteriori, de interesse, sejam calculadas. 


\section{CONCLUSÕES}

No desenvolvimento do método Bayesiano proposto para as análises individuais, admitiu-se que as observações eram provenientes de populações normais independentes com priori não-informativa para os parâmetros do modelo. Embora esta metodologia tenha sido desenvolvida para analisar experimentos balanceados, o desbalanceamento intra experimentos não constitui um problema muito sério. Este poderá ser contornado, adequando-se as expressões das somas dos quadrados, graus de liberdade associados e coeficientes das funções lineares associadas às hipóteses de interesse.

A metodologia Bayesiana desenvolvida, mostrou-se adequada para analisar um grupo de experimentos inteiramente casualizados balanceados. A metodologia permitiu que densidades a posteriori para os parâmetros do modelo e funçōes lineares deles, fossem determinadas e probabilidades a posteriori de interesse fossem calculadas. Além disso, foram determinadas algumas das características de cada distribuição (média, variância, moda, mediana) e intervalos de credibilidade para os parâmetros e funções lineares destes, permitindo, assim, uma grande facilidade de interpretação dos resultados das análises.

A metodologia Bayesiana proposta neste trabalho, apesar de ter considerado que o pesquisador nada conhece acerca do comportamento probabilístico dos parâmetros do modelo, isto é, que nenhum conhecimento a priori foi incorporado na análise, é ainda mais recomendável que uma análise clássica, pois, apresenta, dentre outras, as seguintes vantagens:

- é mais fácil de interpretar os resultados; 
- não necessita aplicar métodos de comparações múltiplas, os quais são bastante discutíveis;

- permite calcular probabilidades a posteriori de um tratamento ser melhor que outro ou de um subgrupo de tratamentos ser melhor que outro subgrupo;

- não fornece estimativas de componentes da variância negativos e os limites inferiores dos intervalos com $(1-\alpha)$ de credibilidade para os componentes de variância serão sempre maiores que ou iguais a zero.

Para realização de uma análise Bayesiana, nos moldes da análise proposta neste trabalho, recorre-se a um volume de cálculos superior aos necessários para realização de uma análise clássica. Além disso, não se tem, ainda, um software específico que faça todas as contas de uma análise Bayesiana, automaticamente. 


\section{REFERÊNCIAS BIBLIOGRÁFICAS}

Bakman, O. R. : Neto, O. C. Análise estatística de decisão. São Paulo: Edgard Blucher, 1980. 124p.

BARNARD, G. A. Thomas Bayes - a bibliographical note. Biometrika, v. 45, p.293-5, 1958.

Bartlett, M. S. Properties of sufficiency and statistical tests. Proceeding Royal Society of London., Séries A, v. 160, p. 268-74, 1937.

Berger, J., ed. Bayesian analysis. Amsterdan, Nort-Holland, 1994. (Special issue journal of statistical planning and inference 40(2/3) 1994).

Bernardo, J. M. ; Smith, A. F. M. Bayesian theory. New York: John Wiley. 1994. 586p.

Besag, J. ; Higdon, D. Bayesian inference for agricultural field experiments. In: ISI, 49., Firenze, 1993. Proceedings.

Box, G. E. P. Non-normality and tests on variances. Biometrika, v. 40, p. 318-35, 1953.

Box, G. E. P. Some theorems on quadratics forms applied in the study of analysis of variance problems. The Annals of Mathematical Statistics, v. 25, p. $290-302,1954$ 
Box, G. E. P. ; TiAO, G. C. A Bayesian approach to the inportance of assumptions applied to the comparision of variances. Biometrika, v. 51, p. 153-64, 1964.

Box, G. E. P. ; TiaO, G. C. Multiparameter problems from a Baysian point of view. The Annals of Mathematical Statistcs, v. 36, p. 1468-82, 1965.

Box, G. E. P. ; TiAo, G. C. Bayesian estimation of means for the random effect model. Journal of the American Statistal Association, v. 63, p. 174-81, 1968.

Box, G. E. P. ; TiaO, G. C. Bayesian inference in analysis. New York: Addison Wesley, 1973. 588p.

Broemeling, L. D. Bayesian analysis of linear models. New York: Marcel Dekker, 1985. 454p.

CAmpos, H. Estatística aplicada a experimentação com cana-de-açúcar. Piracicaba: FEALQ, 1984. 291p.

Char, B. W., Geddes, K. O.; Gonnet, G. H. ; Leong, B. L. Maple V: library reference manual. New York: Springer-Verlag, 1991. 698p.

Clarke, B. ; Wasserman, L. Noninformative priors and nuisace parameters. Journal of the American Statistical Association, v. 88, p.1427-32, 1993.

Cochran, W. G. The combination of estimates from different experiments. Biometrics, v. 10, p. 101-29, 1954.

Cochran, W. G. ; Cox, G. M. Experimental designs. 2. ed., New York: John Wiley, 1957. 611p.

Cochran, W. M. Problems arising in the analysis of a serie of similar experiments. Journal of the Royal Statistical Society, v. 4, p. 102-18, 1937. 
DeGroot, M. H. Probbabilidad e estadistica. San Marcos: Addison-Wesley, 1988. 694p.

Draper, N. R. ; Guttman, I. Unequal group variances in the fixed-effects oneway analysis of variance: a Bayesian sidelight. Biometrika, v. 53, p. 27-35, 1966.

Gomes, F. P. Curso de estatística experimental. 11. ed. Piracicaba: Nobel. 1985. $46 \mathrm{p}$.

Gomez, K. A. ; Gomez, A. A. Statistical procedures for agricultural research. New York: John Wiley, 1984. 680p.

Graybill, F. A. Theory and application of the linear model. Massachusetts: Duxbury Press, 1976. 704p.

Hogg, R.V. ; CraIg, A. T. Introduction to mathematical statistics. New York: Macmillan, 1989. 438p.

Jeffreys, H. Theory of probability. 3. ed. Oxford: Claredon Press, 1961, $578 \mathrm{p}$.

Kempthorne, O. Design and analysis of experiments. 6. ed. New York: Krieger, 1973. 631p.

LAUd, P. H. : IBrahin, J. G. A predictive approach to the analysis of designed experiments. Journal of the American Statistical Association, v. 89, p. 309-19, 1994.

Leandro, R. C. Introdução à inferència bayesiana. Piracicaba, 1993. 50p. (seminário apresentado no curso de pos-graduação em estatística e experimenta ção agronòmica) Escola Superior de Agricultura "Luiz de Queiroz" da Universidade de São Paulo. 
LEE, P. M. Bayesian statistics: an introduction. London: Edwadr Arnold, 1989. 294p.

LINDLEY, D. V. Introduction to probability and statistics from a bayesian view point. Cambrige: University Press., 1965, v. 2, 291p.

Lindley, D. V. Bayesian statistics - a review. Philadelphia: Society for Industrial and Applied Mathematics, 1972. 83p.

LISEO, B. Elimination of nuisance parameters with reference priors. Biometrika, v. 80, p. 295-304, 1993.

Mood, A. M. ; Graybill, F. A. ; Boes, D. C. Introduction to the theory of statistics. 3. ed. Singapore: McGraw-Hill, 1974. 564p.

O'HAGAN, A. Kendall's advanced theory of statistics: Bayesian inference. London: Edward Arnold, 1994. v. 2B, 330p.

OWEN, R. J. The optimum design of a two-factor experiment using priori information. The Annals Mathematical Statistics. v. 41, p. 1917-34, 1970.

Pereira, C. A. B. ; Viana, M. A. Elementos de inferência bayesiana. In: Simpósio de Probabilidade e Estatística, 5. São Paulo, 1982. 98p.

Portioy, S. Formal Bayes estimation with application to a random effects model. The Annals of Mathematical Statistics. v. 42, p. 1379-402, 1971.

Raiffa, H. ; Schlaifer, R. Applied statistical decision theory. Boston: Harvard College, 1961. 355p.

SAmiuddin, M. Bayesian test of homogeneity of variances. Journal of the American Statistical Association, v. 71, p. 515-7, 1976.

Searle, S. R., Casella, G. ; MacCulloch, C. E. Variance components. New York: John Wiley, 1992. 501p. 
Srmões, N. R. Estimadores de Bayes no Modelo de Regressão Linear Simples. Piracicaba, 1977. 138p. (Dissertação Mestrado) Escola Superior de Agricultura "Luiz de Queiroz" da Universidade de São Paulo.

StUart, A. ; ORD, K. Kendall's advanced theory of statistics: distributions theory. 6. ed. New York: Edward Arnold, 1994. v. 1, 676p.

Tiao, G. C. ; Draper, N.R. Bayesian analysis of linear models with special reference to the balanced incomplete block design. Biometrika, v. 55, p. 10117. 1968.

Tiao, G. C. ; Guttman, I. The inverted Dirichlet distribuition with applications, Journal of the American Statistical Association. v. 60, p. 792, 1965.

TIAO, G. C. : TAN, W. Y. Bayesian analysis of random-effect models in the analysis of variance. Posterior distribution of variance components. Biometrika, v. 52, p. $37-53,1965$.

Tiao, G. C. : Zellner, A. Bayes's theorem and the use of prior knowledge in regression analysis. Biometrika. v. 51, p. 219-30, 1964.

Yates, F. ; Cochran, W. G. The analysis of groups of experiments. Journal of Agricultural Science, v. 28, p. 556-80, 1938.

YE, K. : Berger, J. O. Noninformative priors for inferences in experimental regression models. Biometrika. v. 78, p. 645-56, 1993.

ZACKS, S. The Theory of statistical inference. New York: John Wiley, 1971. $609 \mathrm{p}$.

ZELLNER, A. An introduction to Bayesian inference in econometrics. New York: John Wiley, 1971. 431p.

Wolfram, S. Mathematica: a system for doing mathematics by computer. 2. ed. New York: Addison Wesley, 1991. 961p. 
6 APÊNDICE 
COMANDOS PARA O "MATHEMATICA" PARA OBTENÇÃO DOS GRAFICOS DAS DENSIDADES A POSTERIORI DO ERRO EXPERIMENTAL DE CADA ENSAIO E ALGUMAS CARACTERÍSTICAS DAS DISTRIBUIÇÕES.

(a) Usina N. S. Maravilhas

(b) Usina Santa Tereza

(c) Usina São José

(d) Gráfico Conjunto Para os Três Erros.

ps $1=\mathrm{P} \operatorname{lot}\left[\left(((28.0065) \wedge(10.5)) /\left((9.5 !) *\left(2^{\wedge} 10.5\right)\right)\right)\right.$ * $\left(x^{\wedge}(-11.5)\right) *(\operatorname{Exp}[-(28.0065) /(2 * x)]),\{x, 0.001,6\}$, PlotRange->All, AxesLabel->\{" ", "pl"\}, PlotLabel->"(a)"]

$\mathrm{pl}$

(a)

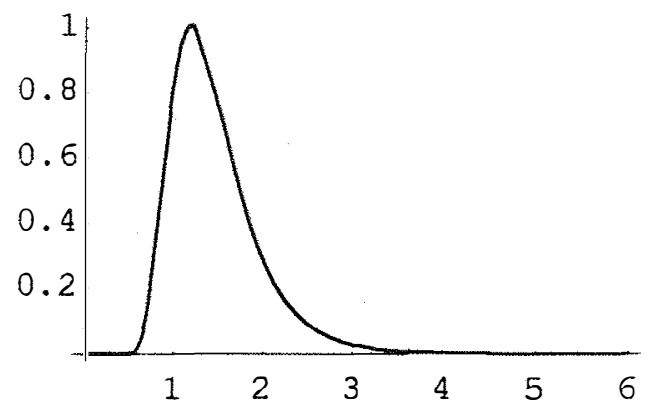

-Graphics-

ps2 $=$ Plot $\left[\left(((52.8705) \wedge(10.5)) /\left((9.5 !) *\left(2^{\wedge} 10.5\right)\right)\right)\right.$ * $\left(x^{\wedge}(-11.5)\right) *(\operatorname{Exp}[-(52.8705) /(2 * x)]),\{x, 0.001,6\}$, PlotRange->All, AxesLabel->\{" ", "p2"\}, PlotLabel->" (b) "] p2

(b)

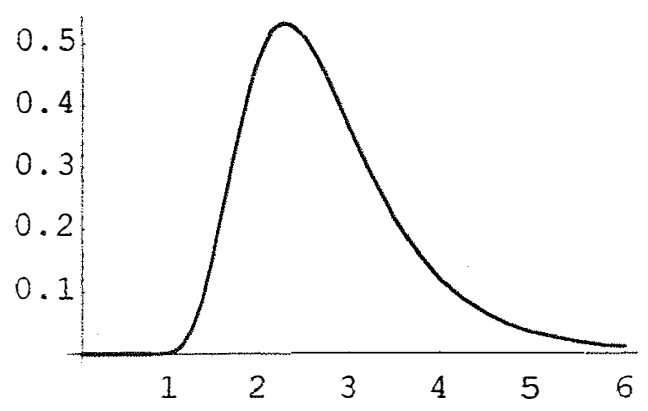

-Graphics- 
ps $3=\operatorname{Plot}\left[\left(\left((11.4174)^{\wedge}(10.5)\right) /\left((9.5 !) *\left(2^{\wedge} 10.5\right)\right)\right) *\right.$

$\left(x^{\wedge}(-11.5)\right) *(\operatorname{Exp}[-(11.4174) /(2 * x)]),\{x, 0.001,6\}$,

PlotRange->All, AxesLabel->\{" ", "p3"\}, PlotLabel->" (c) "] p3

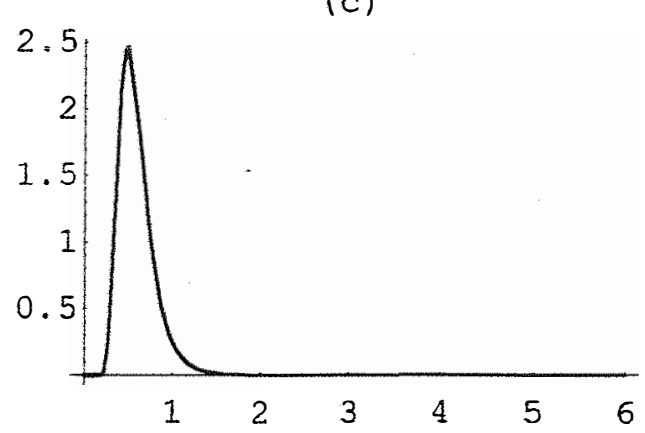

-Graphics-

$g g=$ Show $[p s 1, p s 2, p s 3$, AxesLabel->\{" " , "p" $\}$, PlotLabel->" (d)"]

$\mathrm{p}$

(d)

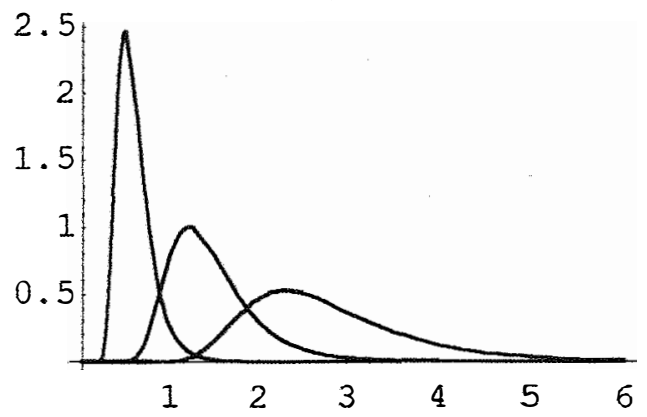

-Graphics- 
Show [GraphicsArray [ \{ $\{p s 1, p s 2\},\{p s 3, g g\}\}]]$

p1

(a)

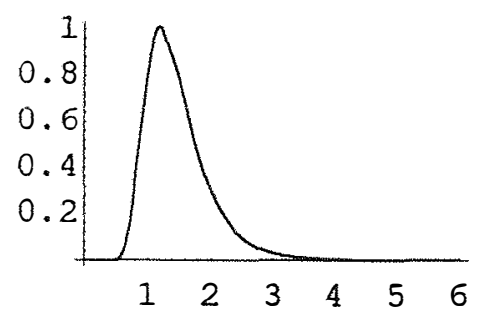

p3

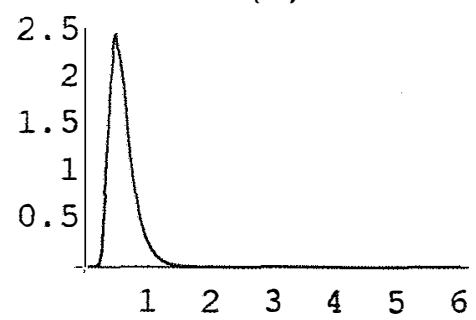

p2

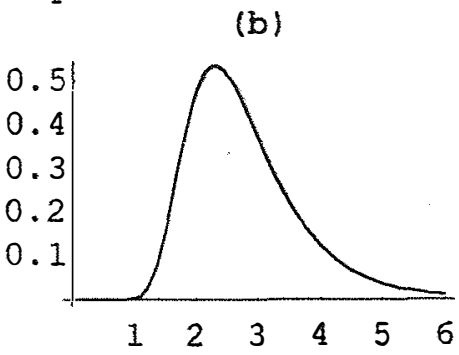

$\mathrm{p}$

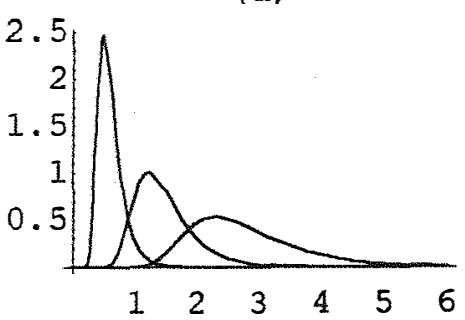

-GraphicsArray-

CÁLCULO DAS MÉDIAS DO ERRO EXPERIMENTAI DE CADA ENSAIO mI $1=N I n$ tegrate $\left[\left(((28.0065) \wedge(10.5)) /\left((9.5 !) *\left(2^{\wedge} 10.5\right)\right)\right) * x^{*}\right.$ $\left.\left(x^{\wedge}(-11.5)\right) *(\operatorname{Exp}[-(28.0065) /(2 * x)]),(x, 0.001,1000\}\right]$

1.47403

$\mathrm{m} 21=\mathrm{NIntegrate}\left[\left(((52.8705) \wedge(10.5)) /\left((9.5 !) *\left(2^{\wedge} 10.5\right)\right)\right) * \mathrm{x}^{*}\right.$ $\left.\left(x^{\wedge}(-11.5)\right) *(\operatorname{Exp}[-(52.8705) /(2 \star x)]),\{x, 0.001,1000\}\right]$

2.78266

m31=NIntegrate $\left[\left(\left((11.4174)^{\wedge}(10.5)\right) /\left((9.5 !) *\left(2^{\wedge} 10.5\right)\right)\right) * x^{*}\right.$ $\left.\left(x^{\wedge}(-11.5)\right) *(\operatorname{Exp}[-(11.4174) /(2 \star x)]),(x, 0.001,1000\}\right]$

0.600916

CÁLCULO DAS E [X^2]

m12=NIntegrate $\left[\left(((28.0065) \wedge(10.5)) /\left((9.5 !) *\left(2^{\wedge} 10.5\right)\right)\right) *\left(x^{\wedge} 2\right)\right.$ * $\left.\left(x^{\wedge}(-11.5)\right) *(\operatorname{Exp}[-(28.0065) /(2 * x)]),(x, 0.001,1000\}\right]$

2.42837

$\mathrm{m} 22=\mathrm{NIntegrate}\left[\left(((52.8705) \wedge(10.5)) /\left((9.5 !) *\left(2^{\wedge} 10.5\right)\right)\right) *\left(x^{\wedge} 2\right)\right.$ * $\left.\left(x^{\wedge}(-11.5)\right) *(E x p[-(52.8705) /(2 * x)]),\{x, 0.001,1000\}\right]$

8.65415

$\mathrm{m} 32=\mathrm{NIntegrate}\left[\left(((11.4174) \wedge(10.5)) /\left((9.5 !) *\left(2^{\wedge} 10.5\right)\right)\right) *\left(x^{\wedge} 2\right)\right.$ * $\left.\left(x^{\wedge}(-11.5)\right) \star(\operatorname{Exp}[-(11.4174) /(2 \star x)]),\{x, 0.001,1000\}\right]$

0.403582 
CÁLCUIO DAS VARIÂNCIAS DO ERRO EXPERIMENTAL

PARA CADA ENSAIO

$v 1=m 12-\left((\operatorname{mi1})^{\wedge} 2\right)$

0.255618

$\mathrm{v} 2=\mathrm{m} 22-\left((\mathrm{m} 21)^{\wedge} 2\right)$

0.910963

$v 3=m 32-\left((\mathrm{m} 31)^{\wedge} 2\right)$

0.0424823

CÁLCULO DA MODA DO ERRO EXPERIMENTAL EM CADA ENSAIO mol=FindMinimum $\left[-\left(\left((28.0065)^{\wedge}(10.5)\right) /\left((9.5 !) *\left(2^{\wedge} 10.5\right)\right)\right)\right.$ * $\left.\left(x^{\wedge}(-11.5)\right) *(\operatorname{Exp}[-(28.0065) /(2 * x)]),\{x,\{0.001,5\}\}\right]$

$\{-1.0071,\{x \rightarrow 1.21767\}\}$

mo2 $=$ FindMinimum $\left[-\left(((52.8705) \wedge(10.5)) /\left((9.5 !) *\left(2^{\wedge} 10.5\right)\right)\right) * x^{*}\right.$ $\left.\left(x^{\wedge}(-11.5)\right) *(\operatorname{Exp}[-(52.8705) /(2 * x)]),\{x,\{0.001,5\}\}\right]$

$\{-1.2825,\{x \rightarrow 2.51764\}\}$

mo3=FindMinimum $\left[-\left(\left((11.4174)^{\wedge}(10.5)\right) /\left((9.5 !) *\left(2^{\wedge} 10.5\right)\right)\right) x^{*}\right.$ $\left.\left(x^{\wedge}(-11.5)\right) *(\operatorname{Exp}[-(11.4174) /(2 * x)]),\{x, 0.001,5\}\right]$

$\{-1.2825,\{x \rightarrow 0.543686\}\}$

CÁICULO DA MEDIANA DO ERRO EXPERTMENTAL EM CADA ENSAIO

Md1=NIntegrate $\left[\left(\left((28.0065)^{\wedge}(10.5)\right) /\left((9.5 !) *\left(2^{\wedge} 10.5\right)\right)\right) *\right.$ $\left.\left(x^{\wedge}(-11.5)\right) *(\operatorname{Exp}[-(28.0065) /(2 * x)]),\{x, 0,1.37718\}\right]$

\subsection{9}

Md2 $=$ NIntegrate $\left[\left(\left((52.8705)^{\wedge}(10.5)\right) /\left((9.5 !) *\left(2^{\wedge} 10.5\right)\right)\right)\right.$ * $\left.\left(x^{\wedge}(-11.5)\right) *(\operatorname{Exp}[-(52.8705) /(2 * x)]),\{x, 0.001,2.5997\}\right]$

0.500005

Md3=NIntegrate $\left[\left(\left((11.4174)^{\wedge}(10.5)\right) /\left((9.5 !) *\left(2^{\wedge} 10.5\right)\right)\right) *\right.$ $\left.\left(x^{\wedge}(-11.5)\right) *(\operatorname{Exp}[-(11.4174) /(2 * x)]),\{x, 0.001 ; 0.56142\}\right]$ 0.500036

INTERVALOS COM 95\% DE CREDIBILIDADE PARA O ERRO EXPERIMENTAI DE CADA ENSAIO

icl=NIntegrate $\left[\left(\left((28.0065)^{\wedge}(10.5)\right) /\left((9.5 !) *\left(2^{\wedge} 10.5\right)\right)\right)\right.$ * $\left.\left(x^{\wedge}(-11.5)\right) *(\operatorname{Exp}[-(28.0065) /(2 * x)]),\{x, 0.7894,2.7236\}\right]$ 0.949996 
ic2 $=$ NIntegrate $\left[\left(\left((52.8705)^{\wedge}(10.5)\right) /\left((9.5 !) *\left(2^{\wedge} 10.5\right)\right)\right)\right.$ * $\left.\left(x^{\wedge}(-11.5)\right) *(\operatorname{Exp}[-(52.8705) /(2 * x)]),\{x, 1.4902,5.1416\}\right]$ 0.95

ic $3=N$ Integrate $\left[\left(\left((11.4174)^{\wedge}(10.5)\right) /\left((9.5 !) *\left(2^{\wedge} 10.5\right)\right)\right)\right.$ * $\left.\left(x^{\wedge}(-11.5)\right) *(\operatorname{Exp}[-(11.4174) /(2 * x)]),\{x, 0.32181,1.11033\}\right]$

0.949999 
LINHAS DE COMANDOS PARA O "MATHEMATICA" PARA OTENÇÃO DOS GRÁEICOS DAS DENSIDADES A POSTERIORI DO ERRO EXPERIMENTAI NA ANÁIISE CONUUNTA, AIGUMAS CARACTERÍSTICAS DA DIST. E OS INTERVALOS COM $95 \%$ DE CREDIBILIDADE.

(a) Posteriori do erro experimental aprox. em um estágio,

(b) posteriori do erro experimental aprox. em dois estágios,

(c) posteriori do erro experimental sem a restição C,

(d) posteriori do erro experimental composto "pooling",

(e) os quatro gráficos juntos.

$p 1=P \operatorname{lot}\left[\left(\left(91.052^{\wedge} 31\right) /\left((30 !) *\left(2^{\wedge} 31\right)\right)\right) *\left(x^{\wedge}(-32)\right)\right.$ *

$(\operatorname{Exp}[-45.5291 / x]),\{x, 0.01,3\}$, PlotRange->AII,

PlotLabel->"(a)",AxesLabel->\{" ", "p1"\}]

p1

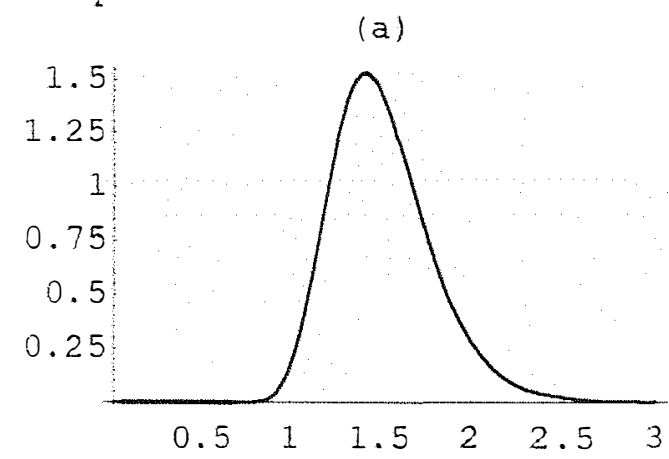

-Graphics-

$\mathrm{p} 2=\mathrm{p} 1$ ot $\left[\left(\left(99.7128^{\wedge} 37\right) /\left((36 !) *\left(2^{\wedge} 37\right)\right)\right) *\left(x^{\wedge}(-38)\right)\right.$ * $(\operatorname{Exp}[-49.8564 / x]),\{x, 0.01,3\}$, PlotRange->All,

PlotLabel->" (b) ",AxesLabel->\{" ", "p2"\}] p2

(b)

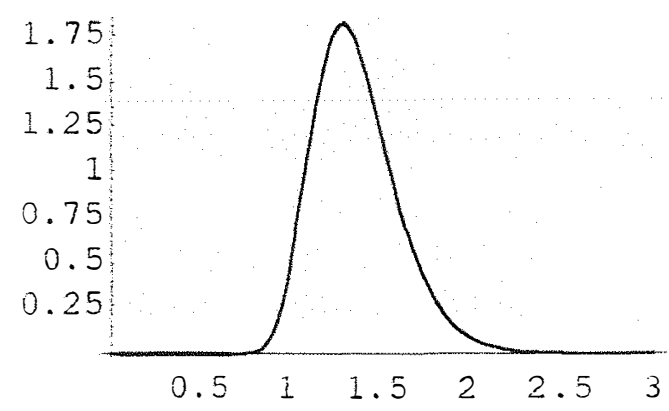

-Graphics- 
$\mathrm{pi}=\mathrm{P} \operatorname{lot}\left[\left(\left(92.2941^{\wedge} 31.5\right) /\left((30.5 !) *\left(2^{\wedge} 31.5\right)\right)\right) *\left(\mathrm{x}^{\wedge}(-32.5)\right) *\right.$ $(\operatorname{Exp}[-46.14705 / x]),\{x, 0.01,3\}, P l o t R a n g e->A 11$, PlotLabel->" (c) ",AxesLabel->\{" ", "pi"\}]

pi

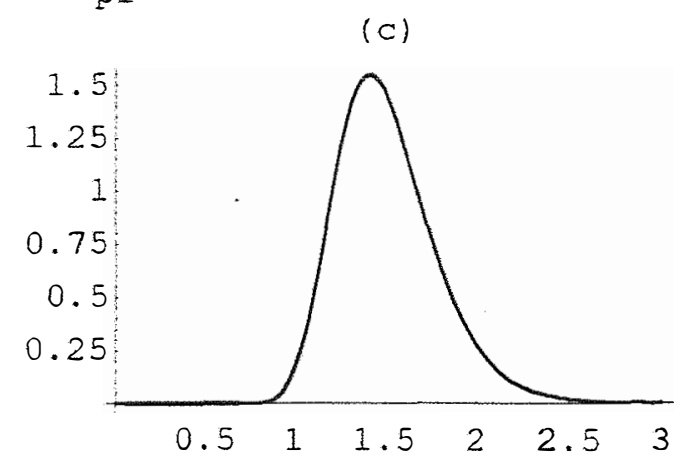

-Graphics-

$\mathrm{pc}=\mathrm{P} 1$ ot $\left[\left(\left(100.2065^{\wedge} 37\right) /\left((36 !) *\left(2^{\wedge} 37\right)\right)\right) *\left(\mathrm{x}^{\wedge}(-38)\right)\right.$ *

$(E \operatorname{Exp}[-50.10325 / x]),\{x, 0.01,3\}$, PlotRange->AII,

PlotLabel->" (d) ",AxesLabel->\{" ", "pc"\}]

pc

(d)

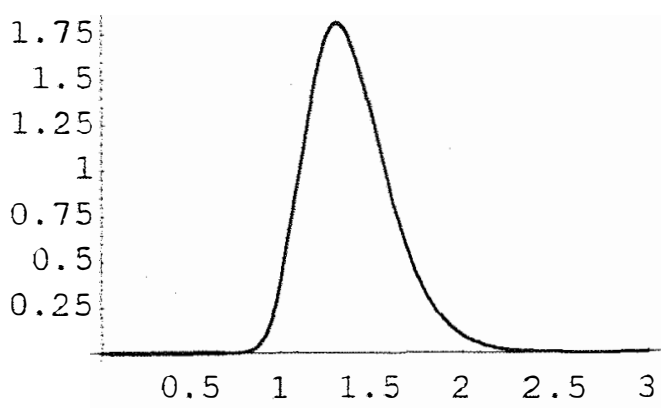

-Graphics- 
Show $[G r a p h i c s A r r a y[\{\{p 1, p 2\},\{p i, p c\}\}]]$

$\mathrm{p} 1$

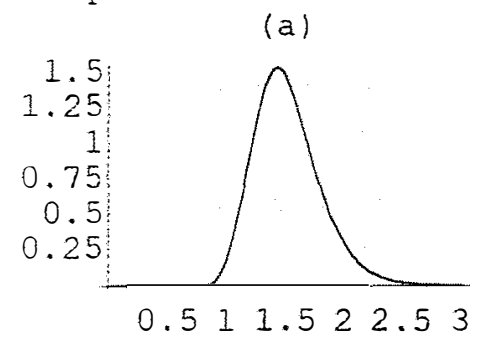

pi

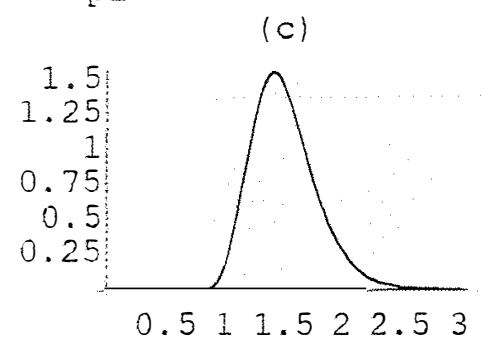

$\mathrm{p} 2$

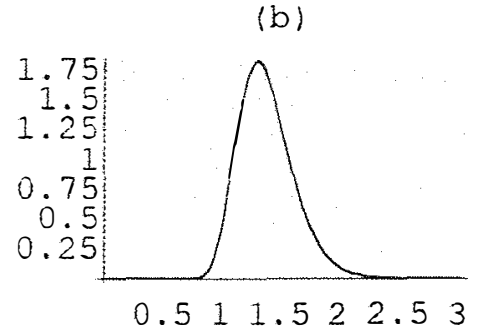

pc

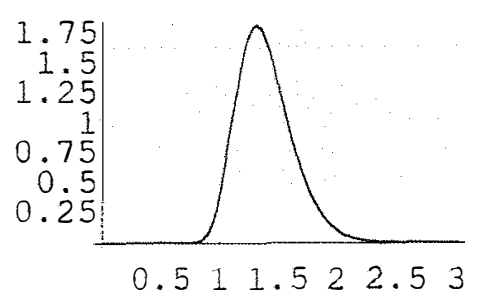

-GraphicsArray-

$p=$ Show $[p 1, p 2, p i, p c$, AxesLabel->\{" ", "p" $\}$, PlotLabel->" (e)"] $p$

(e)

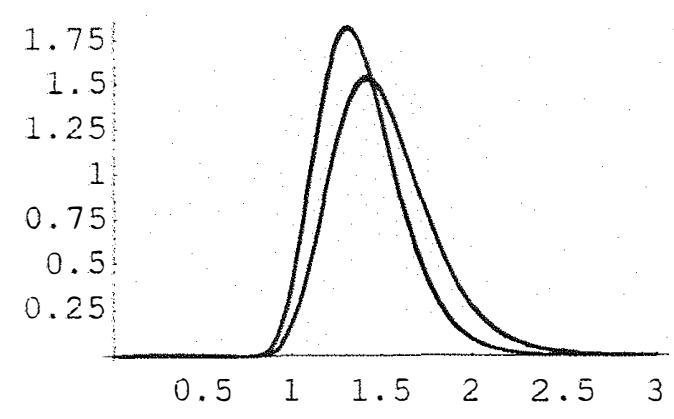

-Graphics-

CÁLCULO DA MÉDIA DO ERRO EXPERIMENTAL, NA ANÁLISE CONJUNTA, USANDO AS DIEERENTES APROXIMAÇÕES PARA A DENSIDADE A POSTERIORI DO ERRO EXPERIMENTAI:

ml=média a partir da posteriori aprox. em um estágio, m2=média a partir da posteriori aprox. em dois estágios, mi=média a partir da posteriori irrestrita, mc=nédia a partir da posteriori composta. 
$m 1=N I n$ tegrate $\left[\left(\left(91.0582^{\wedge} 31\right) /\left((30 !) *\left(2^{\wedge} 31\right)\right)\right) * x^{\star}\left(x^{\wedge}(-32)\right)\right.$ * $(\operatorname{Exp}[-45.5291 / x]),\{x, 0.01,500\}]$

1.51764

$\mathrm{m} 2=\mathrm{NIntegrate}\left[\left(\left(99.7128^{\wedge} 37\right) /\left((36 !) *\left(2^{\wedge} 37\right)\right)\right) * \mathrm{x}^{\star}\left(\mathrm{x}^{\wedge}(-38)\right)\right.$ * $(\operatorname{Exp}[-49.8564 / x]),\{x, 0.01,500\}]$

1.3849

$\mathrm{mi}=\mathrm{NIntegrate}\left[\left(\left(92.2941^{\wedge} 31.5\right) /\left((30.5 !) *\left(2^{\wedge} 31.5\right)\right)\right) * \mathrm{x}\right.$ *

$\left.\left(x^{\wedge}(-32.5)\right) *(E x p[-46.14705 / x]),\{x, 0.01,500\}\right]$

1. 51302

$m c=N I n$ tegrate $\left[\left(\left(100.2065^{\wedge} 37\right) /\left((36 !) *\left(2^{\wedge} 37\right)\right)\right) * x^{*}\left(x^{\wedge}(-38)\right) *\right.$

$(\operatorname{Exp}[-50.10325 / \mathrm{x}]),\{\mathrm{x}, 0.01,500\}]$

1.39176

CÁLCULO DA E $\left[\mathrm{X}^{\wedge} 2\right]$

m12=NIntegrate $\left[\left(\left(91.0582^{\wedge} 31\right) /\left((30 !) *\left(2^{\wedge} 31\right)\right)\right) * x^{\wedge} 2 *\left(x^{\wedge}(-32)\right)\right.$ * $(\operatorname{Exp}[-45.5291 / x]),\{x, 0.01,500\}]$

2.38264

$\mathrm{m} 22=\mathrm{NIntegrate}\left[\left(\left(99.7128^{\wedge} 37\right) /\left((36 !) *\left(2^{\wedge} 37\right)\right)\right) * x^{\wedge} 2^{*}\left(x^{\wedge}(-38)\right)\right.$ * $(\operatorname{Exp}[-49.8564 / \mathrm{x}]),\{\mathrm{x}, 0.01,500\}]$

1.97275

mi2=NIntegrate $\left[\left(\left(92.2941^{\wedge} 31.5\right) /\left((30.5 !) *\left(2^{\wedge} 31.5\right)\right)\right) * x^{\wedge} 2\right.$ *

$\left.\left(x^{\wedge}(-32.5)\right) *(\operatorname{Exp}[-46.14705 / x]),\{x, 0.01,500\}\right]$

2.36682

mc2 $=$ NIntegrate $\left[\left(\left(100.2065^{\wedge} 37\right) /\left((36 !) *\left(2^{\wedge} 37\right)\right)\right) * x^{\wedge} 2 *\left(x^{\wedge}(-38)\right)\right.$ * $(\operatorname{Exp}[-50.10325 / \mathrm{x}]),\{\mathrm{x}, 0.01,500\}]$

1. 99233

CÁLCULO DAS VARIÂNCIAS A POSTERIORI DOS ERRO EXPERIMENTAL USANDO AS DIEERENTES APROXIMAÇÕES PARA POSTERIORI

$\mathrm{v} 1=\mathrm{m} 12-\left((\mathrm{m} 1)^{\wedge} 2\right)$

0.0794214

$\mathrm{v} 2=\mathrm{m} 22-\left((\mathrm{m} 2)^{\wedge} 2\right)$

0.0547985

$v i=m i 2-\left((m i)^{\wedge} 2\right)$

0.0776008 
$\mathrm{vc}=\mathrm{mc} 2-\left((\mathrm{mc})^{\wedge} 2\right)$

0.0553425

CÁICULO DA MODA DO ERRO EXPERIMENTAL USANDO AS DIFERENTES APROXIMAÇÕES PARA POSTERIRI

mol $=$ FindMinimum $\left[-\left(\left(91.0582^{\wedge} 31\right) /\left((30 !) *\left(2^{\wedge} 31\right)\right)\right) *\left(x^{\wedge}(-32)\right)\right.$ * $(\operatorname{Exp}[-45.5291 / \mathrm{x}]),\{\mathrm{x},\{1,3\}\}]$

$\{-1.53259,\{x->1.42278\}\}$

mo2 $=$ FindMinimum $\left[-\left(\left(99.7128^{\wedge} 37\right) /\left((36 !) *\left(2^{\wedge} 37\right)\right)\right) *\left(x^{\wedge}(-38)\right)\right.$ *

$(\operatorname{Exp}[-49.8564 / x]),\{x,\{1,3\}\}]$

$\{-1.82109,\{x \rightarrow>1.31201\}\}$

moi=FindMinimum $\left[-\left(\left(92.2941^{\wedge} 31.5\right) /\left((30.5 !) *\left(2^{\wedge} 31.5\right)\right)\right)\right.$ *

$\left.\left(x^{\wedge}(-32.5)\right) \star(\operatorname{Exp}[-46.14705 / x]),\{x,\{1,3\}\}\right]$

$\{-1.54848,\{x \rightarrow 1.41991\}\}$

moc $=$ FindMinimum $\left[-\left(\left(100.2065^{\wedge} 37\right) /\left((36 !) *\left(2^{\wedge} 37\right)\right)\right)\right.$ *

$\left.\left(x^{\wedge}(-38)\right) *(\operatorname{Exp}[-50.10325 / x]),\{x,\{1,3\}\}\right]$

$\{-1.81211,\{x \rightarrow>1.31851\}\}$

CÁLCULO DA MEDIANA DO ERRO EXPERIMENTAL USANDO AS DIFERENTES APROXIMAÇÕES PARA POSTERIORI

Md1 =NIntegrate $\left[\left(\left(91.0582^{\wedge} 31\right) /\left((30 !) *\left(2^{\wedge} 31\right)\right)\right) *\left(x^{\wedge}(-32)\right)\right.$ * $(\operatorname{Exp}[-45.5291 / x]),(x, 0,1.4848\}]$

0.500278

Md2=NIntegrate $\left[\left(\left(99.7128^{\wedge} 37\right) /\left((36 !) *\left(2^{\wedge} 37\right)\right)\right) *\left(x^{\wedge}(-38)\right)\right.$ *

$(\operatorname{Exp}[-49.8564 / x]),\{x, 0,1.3598\}]$

0.500178

Mdi=NIntegrate $\left[\left(\left(92.2941^{\wedge} 31.5\right) /\left((30.5 !) *\left(2^{\wedge} 31.5\right)\right)\right)\right.$ *

$\left.\left(x^{\wedge}(-32.5)\right) *(\operatorname{Exp}[-46.14705 / x]),\{x, 0,1.4808\}\right]$

0.500265

MdC $=$ NIntegrate $\left[\left(\left(100.2065^{\wedge} 37\right) /\left((36 !) *\left(2^{\wedge} 37\right)\right)\right)\right.$ *

$\left.\left(x^{\wedge}(-38)\right) *(\operatorname{Exp}[-50.10325 / x]),(x, 0,1.3666\}\right]$

0.500297

INTERVALOS COM 95\% DE CREDIBILIDADE PARA O ERRO EXPERIMENTAL 
ICI=NIntegrate $\left[\left(\left(91.0582^{\wedge} 31\right) /\left((30 !) *\left(2^{\wedge} 31\right)\right)\right) *\left(x^{\wedge}(-32)\right)\right.$ * $(\operatorname{Exp}[-45.5291 / x]),(x, 1.0632,2.1620\}]$

0.950022

IC2 $=$ NIntegrate $\left[\left(\left(99.7128^{\wedge} 37\right) /\left((36 !) *\left(2^{\wedge} 37\right)\right)\right) *\left(x^{\wedge}(-38)\right)\right.$ * $(\operatorname{Exp}[-49.8564 / x]),\{x, 1.0004,1.9140\}]$

0.950017

IC $i=N I n t e g r a t e\left[\left(\left(92.2941^{\wedge} 31.5\right) /\left((30.5 !) *\left(2^{\wedge} 31.5\right)\right)\right)\right.$ *

$\left.\left(x^{\wedge}(-32.5)\right) *(\operatorname{Exp}[-46.14705 / x]),(x, 1.0630,2.1491\}\right]$

0.95001

IC $=$ NIntegrate $\left[\left(\left(100.2065^{\wedge} 37\right) /\left((36 !) *\left(2^{\wedge} 37\right)\right)\right)\right.$ *

$\left.\left(x^{\wedge}(-38)\right) *(\operatorname{Exp}[-50.10325 / x]),\{x, 1.0054,1.9236\}\right]$

0.950019 
COMANDOS PARA O "MATHEMATICA" PARA OBTENÇÃO DO GRÁFICO

DA POSTERIORI DO COMPONENTE DA VARIÂNCIA DEVIDO A INTERAÇÃO, ALGUMAS CARACTERÍSTICAS DA DISTRIÇÃO E INTERVALO COM 95\% DE CREDIBILIDADE

$\mathrm{p}=\mathrm{NIntegrate}\left[\left(1 /\left((5.3875 !) *\left(2^{\wedge} 6.3875\right)\right)\right) *\left(\mathrm{x}^{\wedge} 5.3875\right) *\right.$ $(\operatorname{Exp}[-\mathrm{x} / 2]),\{\mathrm{x}, 0,8.896314\}]$

0.233744

psg $=\mathrm{P} l$ ot $\left[\left((4 *((13.0331) \wedge(6.3875))) /\left((5.3875 !) *\left(2^{\wedge} 6.3875\right) *\right.\right.\right.$ $(0.233744))) *((1.465+4 * x) \wedge(-7.3875)) *$

$(\operatorname{Exp}[-(13.0331 /(2.93+8 * x))]),\{x, 0,0.7\}$, PlotRange->All $]$

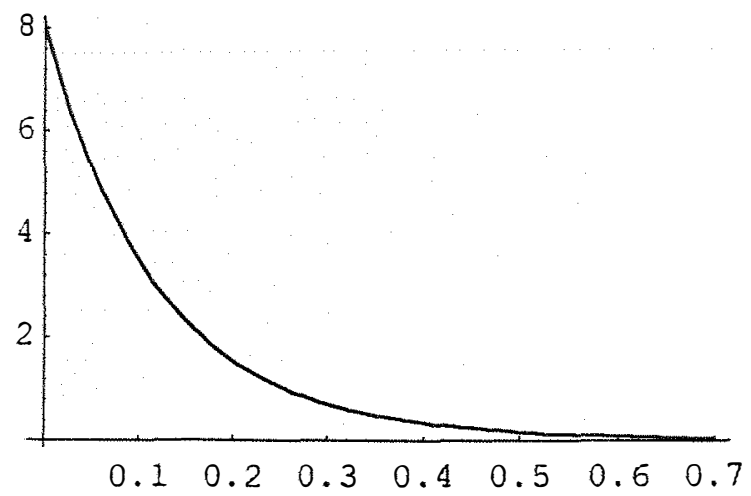

-Graphics-

CÁICULO DA MÉDIA

CÁLCULO DA MÉDIA

$\mathrm{mg}=\mathrm{NIn}$ tegrate $\left[\left((4 *((13.0331) \wedge(6.3875))) /\left((5.3875 !) *\left(2^{\wedge} 6.3875\right)\right.\right.\right.$ * $(0.233744))) * x *((1.465+4 * x) \wedge(-7.3875)) *$

$(\operatorname{Exp}[-(13.0331 /(2.93+8 * x))]),(x, 0,100\}]$

0.135673

CÁLCULO DE E[ $\left[X^{\wedge} 2\right]$

CÁLCULO DE E[X $\left.{ }^{2}\right]$

mg2 $=$ NIntegrate $\left[\left(\left(4 *\left((13.0331)^{\wedge}(6.3875)\right)\right) /\left((5.3875 !) *\left(2^{\wedge} 6.3875\right)\right.\right.\right.$

$(0.233744))) *\left(x^{\wedge} 2\right) *((1.465+4 * x) \wedge(-7.3875)) *$

$(\operatorname{Exp}[-(13.0331 /(2.93+8 * x))]),\{x, 0,100\}]$

0.0425853

CÁLCULO DA VARIÂNCIA

$\mathrm{vg}=\mathrm{mg} 2-\left((\mathrm{mg})^{\wedge} 2\right)$

0.0241782 
A MODA DA DISTRIBUIÇÃO É zero

DETERMINAÇÃO DA MEDIANA

$M d g=N I n$ tegrate $\left[\left(\left(4 *\left((13.0331)^{\wedge}(6.3875)\right)\right) /\left((5.3875 !) *\left(2^{\wedge} 6.3875\right)\right.\right.\right.$ $(0.233744))) *((1.465+4 * x) \wedge(-7.3875)) *$

$(\operatorname{Exp}[-(13.0331 /(2.93+8 * x))]),(x, 0,0.087502\}]$

0.500003

INTERVALO COM $95 \%$ DE CREDIBILIDADE PARA O COMPONETE

DA VARIÂNCIA DEVIDO A INTERAÇÃO

icg=NIntegrate $\left[\left(\left(4 *\left((13.0331)^{\wedge}(6.3875)\right)\right) /\left((5.3875 !) *\left(2^{\wedge} 6.3875\right)\right.\right.\right.$

$(0.233744))) *((1.465+4 * x) \wedge(-7.3875)) *$

$(\operatorname{Exp}[-(13.0331 /(2.93+8 * x))]),(x, 0,0.4248\}]$

0.950001 
COMANDOS PARA O "MATHEMATICA" PARA OBTENÇÃO DO GRÁFICO DA POSTERIRI DO COMPONENTE DA VARIÂNCIA DEVIDO AO FATOR EXPERIMENTO, ALGUMAS CARACTERÍSTICAS DA DISTRIBUIÇÃO E INTERVALO COM 95\% DE CREDIBILIDADE PARA O COMPONENTE DA VARIÂNCIA DEVIDO A EXPERIMENTO.

$\mathrm{p}=\mathrm{NIntegrate}[(1 / 2) *(\operatorname{Exp}[-\mathrm{x} / 2]),\{\mathrm{x}, 0,4.317566\}]$

0.884534

$\mathrm{psb}=\mathrm{P} \operatorname{lot}[((28 * 7.804) /(2 * 0.884534)) *((1.8075+28 * \mathrm{x}) \wedge(-2)) *$ $(\operatorname{Exp}[-(7.804) /(3.615+56 * x)]),\{x, 0,0.6\}$, AxesLabel->\{" ", "psb" $\}]$

psb

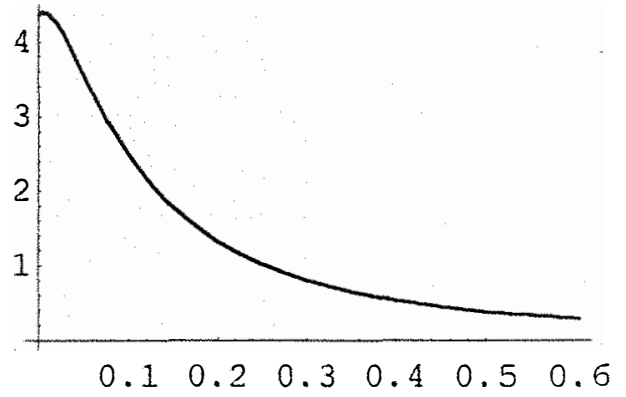

-Graphics-

CÁLCULO DA MÉDIA

mb=NIntegrate $[((28 * 7.804) /(2 * 0.884534)) * x *$

$((1.8075+28 * x) \wedge(-2)) *(\operatorname{Exp}[-(7.804) /(3.615+56 * x)])$ ，

$\{x, 0,1000\}]$

1.23714

CÁLCULO DE E [X^2]

mb2 $=$ NIntegrate $\left[((28 * 7.804) /(2 * 0.884534)) *\left(x^{\wedge} 2\right) *\right.$

$((1.8075+28 * x) \wedge(-2)) *(\operatorname{Exp}[-(7.804) /(3.615+56 * x)])$,

$\{x, 0,1000\}]$

157.19

CÁICULO DA VARIÂNCIA 
$v b=m b 2-\left((m b)^{\wedge} 2\right)$

155.66

A MODA DA DISTRIBUIÇÃO

mob $=$ FindMinimum $[-(123.518) *((1.8075+28 * x) \wedge(-2))$ *

$(\operatorname{Exp}[-(7.804) /(3.615+56 * x)]),\{x,\{0,1\}\}]$

$\{-4.39164,\{x \rightarrow 0.00512489\}\}$

A MEDIANA DA DISTRIBUIÇÃO

mdb=NIntegrate $\left[(123.518) *\left((1.8075+28 * x)^{\wedge}(-2)\right)\right.$ *

$(\operatorname{Exp}[-(7.804) /(3.615+56 * x)]),\{x, 0,0.17413\}]$

0.500011

INTERVALO COM 95\% DE CREDIBILIDADE PARA O COMPONENTE DA

VARIÂNCIA DEVIDO AO FATOR EXPERIMENTO

i $c b=N I n$ tegrate $\left[(123.518) *\left((1.8075+28 * x)^{\wedge}(-2)\right)\right.$ *

$(\operatorname{Exp}[-(7.804) /(3.615+56 * x)]),(x, 0,3.0163\}]$

0.950001 
PROGRAMA SAS PARA AS ANALISES CLASSICAS INDIVIDUAIS

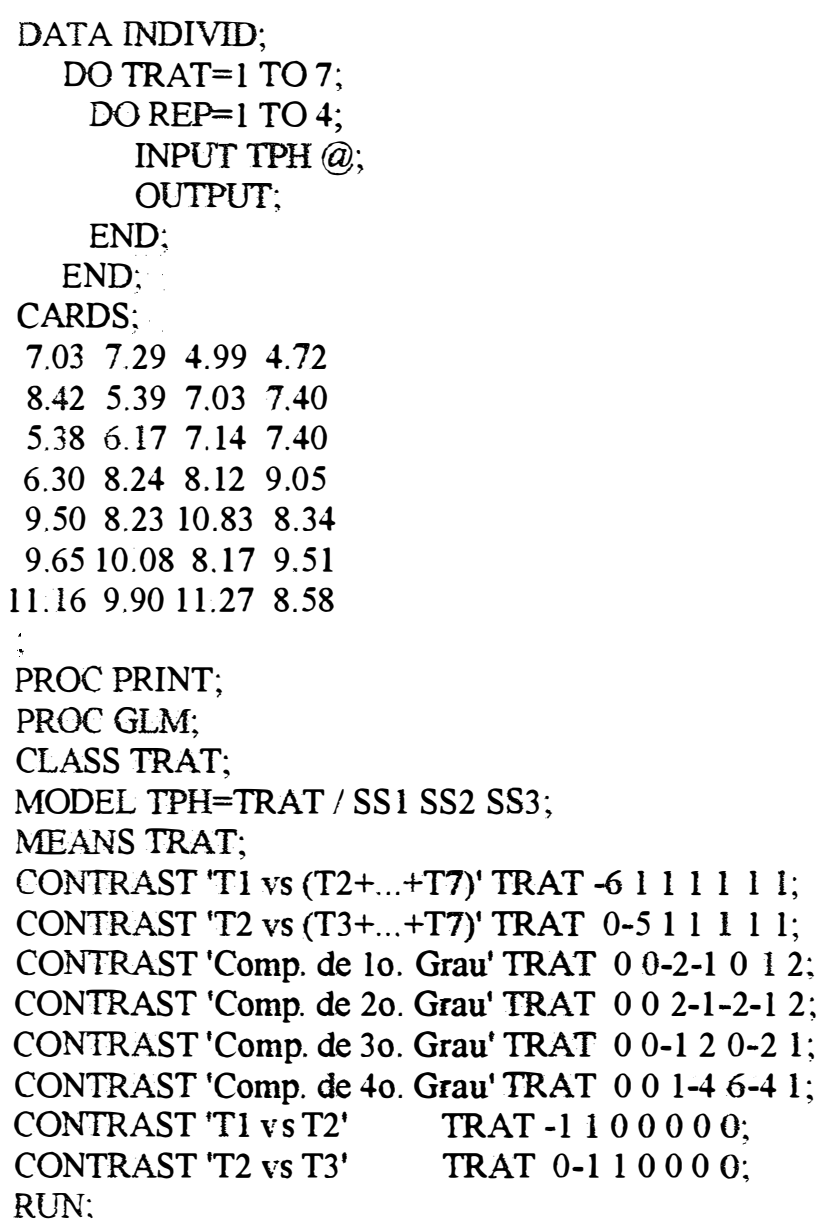


PROGRAMA SAS PARA A ANALISE CONUUNTA CLASSICA

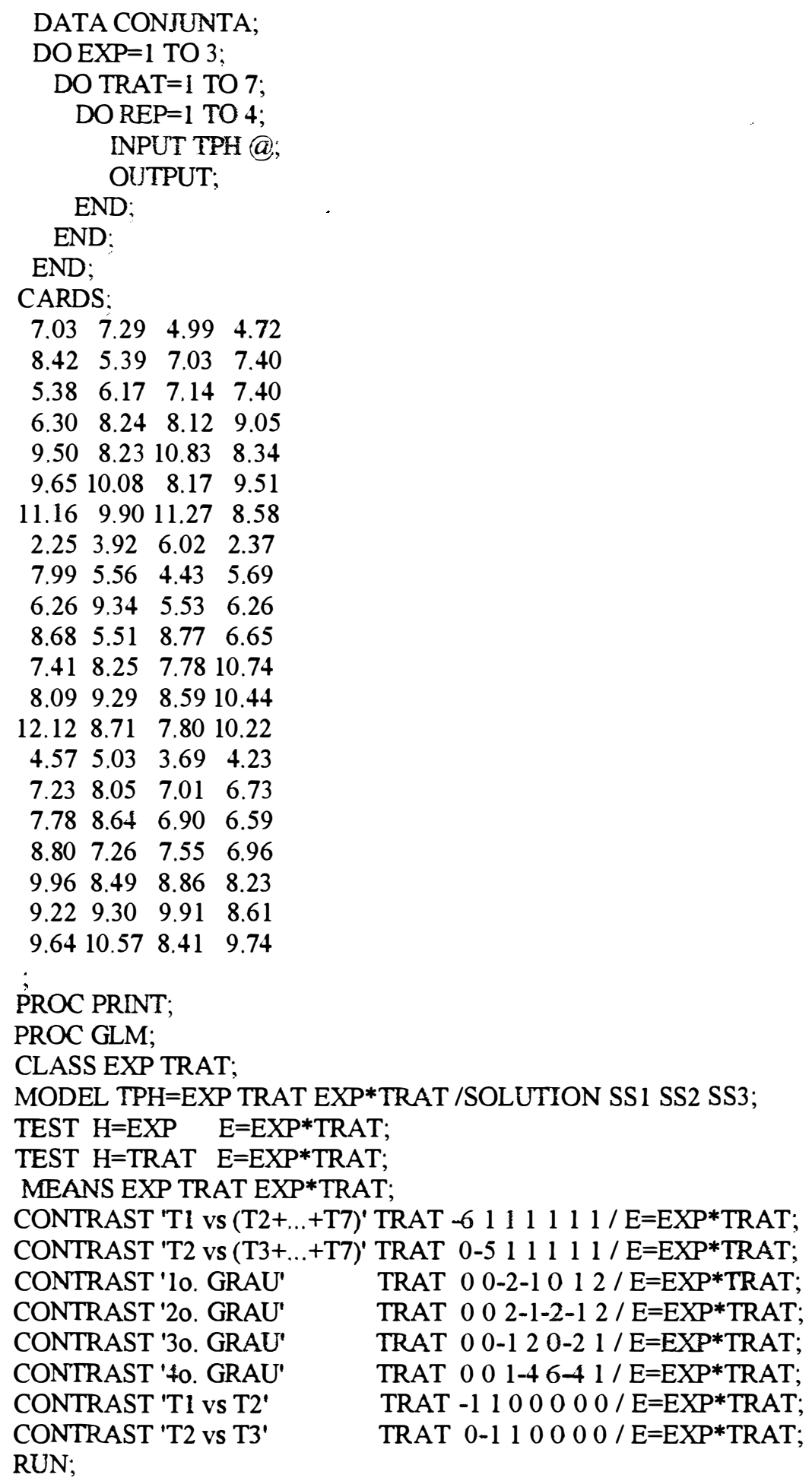


LINHAS DE COMANDOS PARA O SOFTWARE "MATHEMATICA"

USADAS NO CÁLCULO DOS LIMITES INEERIORES E SUPE-

RIORES DAS DISTRIBUIÇÕES DE QUI-QUADRADO E

E-SNEDECOR, PARA CONSTRUÇÃO DOS INTERVALOS

DE CONEIANÇA DOS COMPONENTES DA VARIÂNCIA

NA ANÁLISE CONJUNTA.

$\mathrm{X}=\mathrm{N}$ Integrate $\left[\left(1 /\left((30.5 !) *\left(2^{\wedge} 31.5\right)\right)\right) *\left(\mathrm{x}^{\wedge} 30.5\right) *(\operatorname{Exp}[-\mathrm{x} / 2])\right.$, $\{x, 42.9503,86.8296\}]$

0.95

$x 1=N I n$ tegrate $\left[(1 / 7680) *\left(x^{\wedge} 5\right) *(\operatorname{Exp}[-x / 2])\right.$, $\{x, 3.7525,25.5305\}]$

0.975

$x 2=N I n$ tegrate $[(0.5) * \operatorname{Exp}[-x / 2],\{x, 0.02516,8.764\}]$

0.974998

f1=NIntegrate $\left[(1 /\right.$ Beta $[6,31.5]) *\left((12 / 63)^{\wedge} 6\right)$ * $\left(f^{\wedge} 5\right)$ *

$\left.\left((1+(12 / 63) \star f)^{\wedge}(-37.5)\right),\{f, 0.29835,2.4024\}\right]$

0.974999

f2=NIntegrate $\left[(1 / \operatorname{Beta}[1,6]) *(1 / 6) *\left((1+(1 / 6) * f)^{\wedge}(-7)\right)\right.$,

$\{f, 0.012592,6.4547\}]$

0.975

$x 11=N I n$ tegrate $\left[(1 / 7680) *\left(x^{\wedge} 5\right) *(\operatorname{Exp}[-x / 2])\right.$,

$\{x, 4.40379,23.3367\}]$

0.95

$x 22=N I n$ tegrate $[(0.5) * \operatorname{Exp}[-x / 2],\{x, 0.050636,7.3778\}]$

0.95

f11=NIntegrate $\left[(1 /\right.$ Beta $[6,31.5]) *((12 / 63) \wedge 6) *\left(f^{\wedge} 5\right)$ *

$\left.\left((1+(12 / 63) * f)^{\wedge}(-37.5)\right),\{f, 0.351853,2.15811\}\right]$

0.95

f22=NIntegrate $[(1 / \operatorname{Beta}[1,6]) *(1 / 6) *((1+(1 / 6) * f) \wedge(-7))$,

$\{f, 0.025371,5.0959\}]$

0.950001 